\title{
ALGORITMOS PARA COMPARAÇÃO \\ DE ÁRVORES FILOGENÉTICAS \\ E O PROBLEMA DOS \\ PONTOS DE RECOMBINAÇÃO
}

Estela Maris Rodrigues

TESE APRESENTADA

$\mathrm{AO}$

INSTITUTO DE MATEMÁTICA E ESTATÍSTICA

DA

UNIVERSIDADE DE SÃO PAULO

PARA

OBTENÇÃO DO GRAU

DE

DOUTOR EM MATEMÁTICA APLICADA

Área de Concentração: Ciência da Computação

Orientadora: Profa. Yoshiko Wakabayashi

Durante a elaboração deste trabalho a autora recebeu apoio financeiro do CNPq

- São Paulo, 17 de fevereiro de 2003 - 


\title{
ALGORITMOS PARA COMPARAÇÃO DE ÁRVORES FILOGENÉTICAS E O PROBLEMA DOS PONTOS DE RECOMBINAÇÃO
}

\author{
Estela Maris Rodrigues
}

Este exemplar corresponde à redação final da tese devidamente corrigida e defendida por Estela Maris Rodrigues e aprovada pela Comissão Julgadora.

São Paulo, 17 de março de 2003.

Comissão Julgadora:

- Profa. Dra. Yoshiko Wakabayashi (orientadora) - IME-USP, Brasil

- Profa. Dra. Marie-France Sagot - INRIA Rhône-Alpes, França

- Prof. Dr. José Augusto Ramos Soares - IME-USP, Brasil

- Prof. Dr. Eduardo Sany Laber - PUC-Rio, Brasil

- Prof. Dr. João Meidanis - IC-UNICAMP, Brasil 


\section{Resumo}

Uma árvore filogenética $\mathcal{T}$ é uma árvore enraizada cujas folhas estão distintamente rotuladas com elementos de um conjunto finito $S_{\mathcal{T}}$, e cujos nós internos têm grau pelo menos 2. Uma floresta de concordância (agreement forest) de duas árvores filogenéticas $\mathcal{T}$ e $\mathcal{U}$ tais que $S_{\mathcal{T}}=S_{\mathcal{U}}$ é uma floresta filogenética que pode ser obtida tanto a partir de $\mathcal{T}$ quanto de $\mathcal{U}$ realizando-se uma seqüência do seguinte par de operações: remoção de um arco e contração do arco cuja extremidade superior incide no arco removido. Neste trabalho, estudamos o seguinte problema: dadas duas árvores filogenéticas $\mathcal{T}$ e $\mathcal{U}$ tais que $S_{\mathcal{T}}=S_{\mathcal{U}}$, encontrar uma floresta de concordância ótima, isto é, uma floresta de concordância de $\mathcal{T}$ e $\mathcal{U}$ que tenha um número mínimo de componentes. Este problema tem relação com o problema da localização de pontos de recombinação em seqüências genéticas ou protêicas pré-alinhadas, para o qual um modelo foi apresentado por Hein $[17,18]$.

Os resultados prévios para este problema, ambos para árvores filogenéticas com grau no máximo 2 nos nós, consistem em uma prova de que o problema é NP-difícil e um algoritmo de aproximação devido a Hein et al. $[19,20]$. Em nosso trabalho, mostramos que o Algoritmo de Hein et al., apresentado como uma 3-aproximação, possui na verdade razão de aproximação 4, e que essa razão é justa. Também propomos, implementamos e testamos três algoritmos de 3-aproximação para o problema. Estes algoritmos diferem consideravelmente entre si com relação aos métodos de análise empregados e também quanto à qualidade das soluções produzidas. Esses resultados de aproximabilidade são desenvolvidos no contexto de uma família de algoritmos para o problema da floresta de concordância ótima; limitantes superiores e inferiores são apresentados para as razões de aproximação desses algoritmos. Adicionalmente, estendemos um desses algoritmos de 3-aproximação para um algoritmo de $(d+1)$-aproximação para o problema da floresta de concordância ótima com árvores filogenéticas possuindo grau no máximo $d$ nos nós $(d \geq 2)$. Desenvoivemos ainda uma prova de que o problema da floresta de concordância ótima é APX-difícil para árvores com grau no máximo 2 utilizando idéias de Hein et al. $[19,20]$. Este resultado, junto com a $(d+1)$-aproximação para o problema da floresta de concordância ótima para árvores com grau no máximo $d$ que provamos, mostra que este último problema é APX-completo e que o problema da floresta de concordância ótima para árvores sem restrição nos graus dos nós é APX-difícil.

A relação entre o problema da floresta de concordância ótima e o modelo de Hein é feita através do problema de encontrar, para duas árvores filogenéticas com o mesmo conjunto de rótulos nas folhas, o número mínimo de transferências de subárvore que transforma uma árvore na outra. Allen e Steel [1] observam que as distâncias entre árvores filogenéticas dadas pelo número mínimo de transferências de subárvore (SPR) e pelo número de componentes de uma floresta de concordância ótima (MAF) não são equivalentes, corrigindo uma observação anterior de Hein et al. [20]. Em nosso trabalho, definimos duas distâncias entre árvores filogenéticas baseadas em diferentes tipos de transferências de subárvore. Mostramos que uma dessas distâncias equivale à distância MAF, enquanto que a outra é igual a MAF em alguns casos e MAF -1 nos demais. Esta segunda distância pode ser aplicada ao cálculo do número de eventos de recombinação no modelo de Hein, pois as transferências de subárvore permitidas por ela equivalem a esses eventos. 


\begin{abstract}
A phylogenetic tree $\mathcal{T}$ is a rooted tree whose leaves are uniquely labeled with elements of a finite set $S_{\mathcal{T}}$, and whose internal nodes have degree at least 2. An agreement forest of two phylogenetic trees $\mathcal{T}$ and $\mathcal{U}$ such that $S_{\mathcal{T}}=S_{\mathcal{U}}$ is a forest that can be obtained from $\mathcal{T}$ and $\mathcal{U}$ by performing a sequence of the following pair of operations: deletion of an arc and contraction of the arc whose upper endpoint is incident with the arc that was deleted. In this work, we study the following problem: given two trees $\mathcal{T}$ and $\mathcal{U}$ such that $S_{\mathcal{T}}=S_{\mathcal{U}}$, find a maximum agreement forest, that is, an agreeement forest of $\mathcal{T}$ and $\mathcal{U}$ which has a minimum number of components. This problem relates to the problem of locating recombination points in a group of pre-aligned sequences, for which a model was proposed by Hein $[17,18]$.
\end{abstract}

Previous results for the maximum agreement forest problem for trees with node degrees bounded by 2 comprise an NP-hardness proof and an approximation algorithm by Hein et al. $[19,20]$, claimed to have performance ratio 3 . In this work we show that Hein's algorithm has performance ratio 4 (a sharp bound), and propose three new 3-approximation algorithms for the problem. All these algorithms have been implemented, and results of computational experiments are reported. Both the analysis techniques we used and the computational results we obtained are very different for each of these algorithms. These approximability results are presented in the context of a family of algorithms for the maximum agreement forest problem; upper and lower bounds are provided for the performance ratio of these algorithms. We have also extended one of our 3-approximation algorithms to a $(d+1)$ approximation algorithm for the maximum agreement forest problem for trees with degrees bounded by $d(d \geq 2)$, and developed an APX-hardness proof for trees with degrees bounded by 2 based on ideas from Hein et al. $[19,20]$. This result, combined with our $(d+1)$ approximation algorithm for the maximum agreement forest problem for trees with bounded degree $d$, proves that this problem is APX-complete and that the maximum agreement forest problem for trees with unbounded degrees is APX-hard.

The maximum agreement forest problem relates to Hein's model through the subtree transfer distance problem. This consists of finding, for a pair of phylogenetic trees $\mathcal{T}$ and $\mathcal{U}$ with degrees bounded by 2 such that $S_{\mathcal{T}}=S_{\mathcal{U}}$, the minimum number of subtree transfer operations that transform one of these trees into the other. Allen and Steel [1] observe that the maximum agreement forest distance (MAF) and the subtree transfer distance (SPR) are not equivalent, therefore correcting a previous remark in Hein et al. [20]. In this work, we define two types of distance for phylogenetic trees based on different types of subtree transfer operations. We show that one of these distances is equivalent to MAF and the other is equal to MAF-2 in some cases and equal to MAF-2 - 1 in the remaining cases. This second distance can be used to calculate the number of recombination events in Hein's model, since there is a one-to-one correspondence between the subtree transfer operations it allows and the recombination events. 


\section{Agradecimentos}

À professora Yoshiko Wakabayashi pela excelente orientação e pelas oportunidades abertas, e também pelo apoio e compreensão, que foram muito importantes para a elaboração e a conclusão deste trabalho.

À professora Marie-France Sagot pelas muitas discussões e sugestões para o aprimoramento deste trabalho, e pela amizade e carinho manifestados.

Aos professores do Instituto de Matemática e Estatística da USP por terem inspirado em mim o gosto pela pesquisa.

Aos funcionários da Comissáo de Pós-Graduação do IME, em especial ao Pinho e ao Feijão, pelo trabalho eficiente e pela gentileza.

Aos colegas do Instituto Pasteur, junto ao qual uma parte deste trabalho foi desenvolvida.

Ao CNPq e à CAPES, pelos auxílios concedidos.

Aos colegas e amigos, em especial à Liliane, à Milkes, ao Paulo, ao Jorge e ao Marko pelo interesse, pela generosidade e pela paciência nas minhas horas menos felizes.

À minha família. 


\section{Índice}

Introdução 1

$\begin{array}{lll}\text { I Preliminares } & 7\end{array}$

1 Noções fundamentais $\quad 8$

1.1 Conjuntos e funções . . . . . . . . . . . . . . . . . . . . . 8

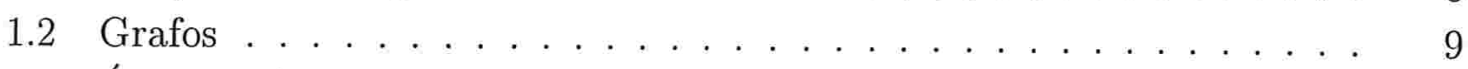

1.3 Árvores e florestas enraizadas . . . . . . . . . . . . . . . 11

2 Classes de problemas, algoritmos e complexidade $\quad 15$

2.1 Conceitos básicos . . . . . . . . . . . . . . . . . 15

2.2 Problemas de decisão . . . . . . . . . . . . . . . . . . . . . . . . . . . . . . . . . . . . . . . .

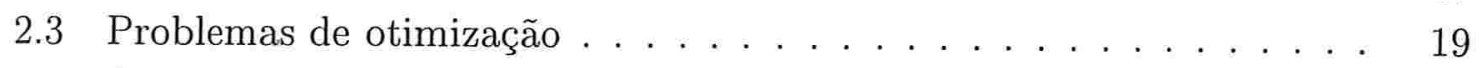

2.4 Algoritmos de aproximação . . . . . . . . . . . . . . . . . 21

\section{0 problema da floresta filogenética de concordância ótima 25}

3 Florestas filogenéticas de concordância $\quad 26$

3.1 Árvores e florestas filogenéticas . . . . . . . . . . . . . . . 26

3.2 Restrições e projeções . . . . . . . . . . . . . . . . . . . . . 27

3.3 Florestas de concordância ótima . . . . . . . . . . . . . . . . . . . . 31

3.4 Arcos de ligação . . . . . . . . . . . . . . . . . . . . . . . . . 34

4 Algoritmos de aproximação para árvores com grau no máximo $2 \quad 36$

4.1 Eliminações, inserções e transferências de subárvore . . . . . . . . . 36

4.2 O método básico . . . . . . . . . . . . . . . . . . . . . . . . . 43

4.3 O algoritmo de aproximação $\mathcal{A}_{\bar{k}} \ldots \ldots \ldots \ldots$. . . . . . . . . . 46

4.3 .1 Transações . . . . . . . . . . . . . . . . . . 46 
4.3.2 Prova da correção do algoritmo . . . . . . . . . . . . . . . 47

4.3.3 Complexidade computacional . . . . . . . . . . . . . 50

4.4 Razão de aproximação do algoritmo $\mathcal{A}_{\bar{k}} \ldots \ldots$. . . . . . . . . . . 51

4.4 .1 Limitantes inferiores . . . . . . . . . . . . . . . . . 51

4.4 .2 Restrições completas e o método básico . . . . . . . . . . . 58

4.4.3 Protocolo de cobrança de taxas . . . . . . . . . . . . 63

4.4.3.1 Protocolo de créditos . . . . . . . . . . . . . 63

4.4.3.2 Protocolo de débitos . . . . . . . . . . . . 66

4.4.3.3 Barreiras ................. 68

4.4 .4 Limitantes superiores . . . . . . . . . . . . . . . . . . . . . . . . . . . . . . . . . .

4.5 O algoritmo de aproximação $\mathcal{B} \ldots \ldots \ldots \ldots$. . . . . . . . . 75

5 Experimentos computacionais $\quad 77$

5.1 O programa e formato dos arquivos de entrada e saída . . . . . . . 77

5.2 Estrutura de dados para florestas filogenéticas . . . . . . . . . . . 80

5.3 Implementação dos algoritmos . . . . . . . . . . . . . . . . . . 86

5.4 Testes e o algoritmo de aproximação $\mathcal{C} \ldots \ldots$. . . . . . . . . 87

6 Algoritmos de aproximação para árvores com grau superior a $2 \quad 96$

7 Resultados de inaproximabilidade $\quad 101$

7.1 O problema da floresta de concordância ótima com grau no máximo 2

é APX-completo. . . . . . . . . . . . . . . . . . . . . 101

\section{O Problema da Recombinação}

8 Transferências de subárvore $\quad 112$

8.1 Transferências canônicas . . . . . . . . . . . . . . . . . . . . 112

8.2 Relação entre seqüências de transferências canônicas e florestas de concordância . . . . . . . . . . . . . . . . . 115

8.3 Conversão de florestas de concordância em seqüências de transferências de subárvore . . . . . . . . . . . . . . . . . . . 121

9 Modelagem de eventos de recombinação 123

9.1 O modelo de Hein para recombinações . . . . . . . . . . . . . . . . . 124

9.2 Transferências equivalentes a eventos de recombinação . . . . . . . . 126

9.2.1 Recombinações que ligam uma restrição em um nó antepassado 129

9.2.2 Recombinações que geram circuitos orientados em redes . . . . 130 
9.3 Transferências padrão . . . . . . . . . . . . . . . . . . . . . 135

9.4 Limitante inferior para a distância padrão . . . . . . . . . . . . 136

9.5 Limitante superior para a distância padrão . . . . . . . . . . . 137

9.5.1 Florestas de concordância induzidas por seqüências de transferências de subárvore . . . . . . . . . . . . . . . . 137

9.5.2 Restrições induzidas por florestas de concordância . . . . . . . 138

9.5.3 Relação entre a distância padrão e a distância canônica . . . . 140 


\section{Introdução}

O grande volume de dados sobre seqüências macromoleculares, oriundos de projetos recentes de sequenciamento de genomas completos, tem renovado o interesse por problemas clássicos em Biologia Computacional. Um desses problemas é o da reconstrução de filogenias a partir de dados sequenciais.

$\mathrm{Na}$ formulação clássica do problema, a entrada é composta de um conjunto finito $S$ de cadeias de caracteres, que representam seqüências genéticas ou protêicas. A saída desejada é uma árvore enraizada $\mathcal{T}$ com nós de grau diferente de 1 e um conjunto $R \supseteq S$ de cadeias, rotulando os nós de $\mathcal{T}$, de modo que as folhas de $\mathcal{T}$ estejam rotuladas com as cadeias em $S$. A árvore $\mathcal{T}$ seria uma representação do processo evolutivo que teria originado as seqüências em $S$, e as cadeias em $R \backslash S$ seriam as seqüências ancestrais, produzidas durante o processo evolutivo que originou as cadeias em $S$. A árvore $\mathcal{T}$ junto com o conjunto $S$ de rótulos nas folhas é denominada árvore filogenética. Se $\mathcal{T}$ é uma árvore filogenética, então seu conjunto de rótulos nas folhas é denotado por $S_{\mathcal{T}}$.

Dados sequenciais, entretanto, estão sujeitos a fenômenos que violam alguns pressupostos básicos dessa formulação, em particular o de que a informação filogenética se difunde na direção das folhas por segregação a partir de um único ancestral comum. Um desses fenômenos é a recombinação, ou seja, a produção de seqüências descendentes pelo crossing-over de duas seqüências antepassadas imediatas. Esse fenômeno produz seqüências descendentes em que ocorre a alternância de trechos herdados de cada uma das seqüências antepassadas imediatas envolvidas no crossing-over. Na presença de eventos de recombinação, o processo evolutivo que produz as seqüências em $S$ não pode mais ser modelado por árvores filogenéticas.

Hein $[17,18]$ propôs um modelo para a análise filogenética de dados sequenciais pré-alinhados relacionados por um processo evolutivo e sujeitos a recombinação. No modelo tradicional, em que não consideramos a ocorrência de eventos de recombinação, supomos que uma única árvore filogenética modela o processo evolutivo das seqüências ao longo de todas as colunas do alinhamento. Hein argumenta que, na presença de recombinação, para cada coluna do alinhamento podemos ter uma árvore filogenética diferente, e que árvores filogenéticas de colunas vizinhas estão relacionadas por operações de transferência de subárvore (subtree transfer), sendo que cada 
ponto de recombinação corresponde a uma transferência de subárvore entre as árvores filogenéticas correspondentes às colunas.

Com base nesse argumento, Hein [17] propôs um método computacional para reconstrução filogenética e localização de pontos de recombinação em seqüências préalinhadas sujeitas a recombinação baseado no cálculo do número mínimo de transferências de subárvore que transforma uma árvore filogenética em outra árvore filogenética. Dadas as limitações deste método por razões de complexidade, uma versão mais simples do método, baseada em uma heurística que evita o cálculo exato do número de transferências de subárvore, foi proposta e implementada no programa RecPars (Hein [18]).

Informalmente, a operação de transferência de subárvore de uma dada árvore $\mathcal{T}$ consiste em realizar as seguintes operações. Primeiramente, remove-se um arco $e$ de $\mathcal{T}$ e contrai-se o arco cuja extremidade superior incide em $e$ obtendo-se duas árvores $\mathcal{T}_{1}$ e $\mathcal{T}_{2}$, sendo $\mathcal{T}_{1}$ a árvore na qual foi feita a contração. Em seguida, insere-se a árvore $\mathcal{T}_{2}$ em um arco de $\mathcal{T}_{1}$, gerando-se um novo nó no ponto de conexão (veja a figura abaixo).
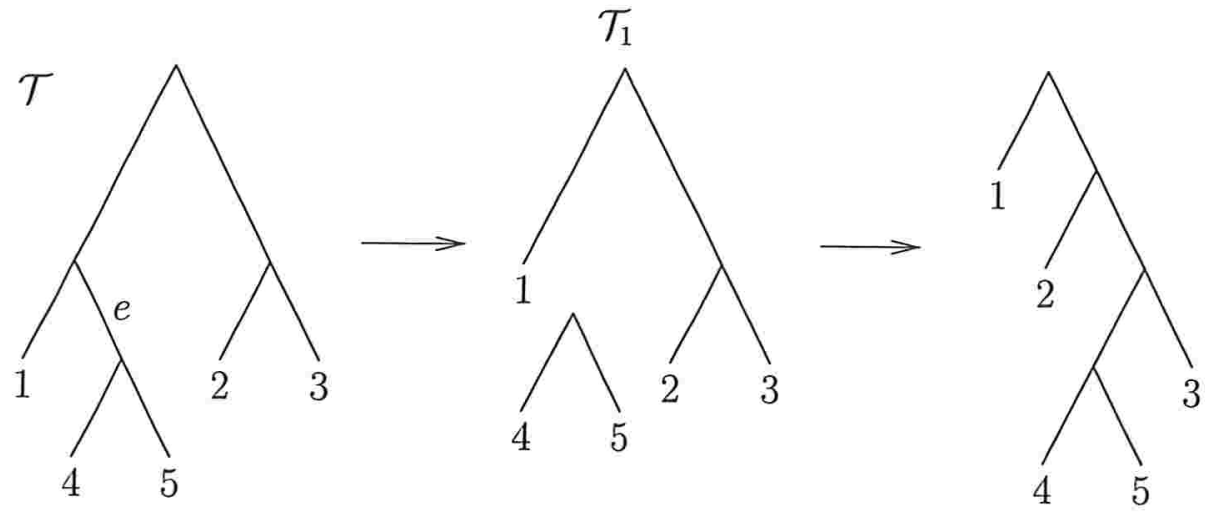

$\mathcal{T}_{2}$

Em nosso trabalho, propomos a incorporação, ao modelo de Hein, de um algoritmo de aproximação para o cálculo do número mínimo de transferências de subárvore. Os resultados de aproximabilidade que vamos apresentar para este problema são derivados de investigações sobre a complexidade e a aproximabilidade do problema da floresta de concordância ótima, o tema central deste trabalho. 


\section{Florestas de concordância ótima}

Uma floresta de concordância (agreement forest) de duas árvores filogenéticas $\mathcal{T}$ e $\mathcal{U}$ tais que $S_{\mathcal{T}}=S_{\mathcal{U}}$ é uma floresta filogenética que pode ser obtida tanto a partir de $\mathcal{T}$ quanto a partir de $\mathcal{U}$ realizando-se uma seqüência do seguinte par de operações: remoção de um arco e contração do arco cuja extremidade superior incide no arco removido (veja a figura abaixo). Uma floresta de concordância de $\mathcal{T}$ e $\mathcal{U}$ é ótima se possui um número mínimo de componentes dentre todas as florestas de concordância de $\mathcal{T}$ e $\mathcal{U}$. Intuitivamente, quanto menos componentes tem a floresta de concordância de $\mathcal{T}$ e $\mathcal{U}$, mais próximas estão essas árvores, relativamente à operação de se transformar $\mathcal{T}$ em $\mathcal{U}$ através de uma seqüência de transferências de subárvore.
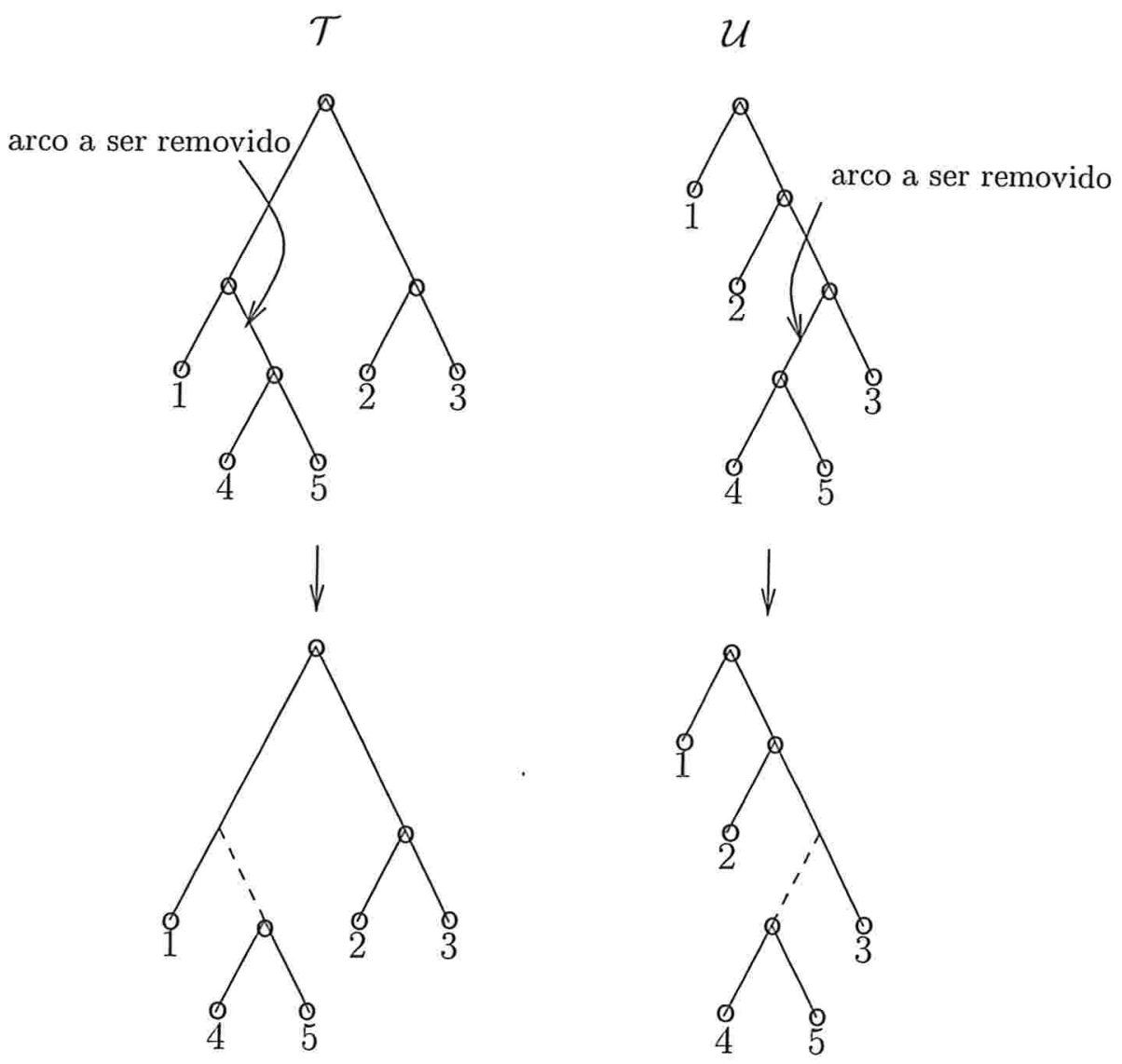

Hein et al. $[19,20]$ argumentam que o problema da floresta de concordância ótima é equivalente ao problema de determinar o número mínimo de transferências de subárvore e apresentam uma prova de que o problema da floresta de concordância ótima é NP-difícil para árvores com grau no máximo 2. Hein et al. $[19,20]$ comen- 
tam ainda que a redução usada nesta prova pode ser facilmente transformada numa prova de que os problemas da floresta de concordância ótima e do número mínimo de transferências de subárvore são MAX SNP-difíceis. Entretanto, a redução apresentada no Teorema 8 de Hein et al. [20] é válida apenas para o problema da floresta de concordância ótima, e o enunciado do Lema 7 de Hein et al. [20], que declara a equivalência entre os dois problemas, está incorreto. Este fato, a ser mostrado na Parte III deste trabalho, foi observado pela primeira vez por Allen e Steel [1]. Adicionalmente, Hein et al. $[19,20]$ apresentam um algoritmo de aproximação para o problema da floresta de concordância ótima para árvores com grau no máximo 2, e alegam (incorretamente) que a razão de aproximação deste algoritmo é 3.

Neste trabalho, apresentamos uma família de instâncias do problema da floresta de concordância ótima para árvores com grau no máximo 2 que mostra que a razão de aproximação do algoritmo proposto por Hein et al. [19, 20] não é inferior a 4. Mostramos ainda que razão de aproximação desse algoritmo é 4 e que essa razão é justa. Apresentamos também três outros algoritmos de aproximação para este problema, e provamos que esses três algoritmos têm razão de aproximação 3. Esses resultados de aproximabilidade são desenvolvidos no contexto de uma família de algoritmos para o problema da floresta de concordância ótima; limitantes superiores e inferiores são apresentados para as razões de aproximação desses algoritmos. Esses três algoritmos e mais o algoritmo de Hein foram implementados e testados com instâncias geradas pela aplicação de um número prescrito de transferências de subárvore a uma árvore filogenética gerada aleatoriamente; foi desenvolvido um utilitário para a geração dessas instâncias. Os três algoritmos de 3-aproximação que propomos diferem consideravelmente entre si nos métodos de análise da razão de aproximação e na qualidade das soluções que os testes computacionais mostraram. Em particular, os testes que fizemos mostram que um dos algoritmos produz soluções de tamanho não superior a 2 vezes o tamanho da solução ótima para instâncias com 1000 folhas geradas executando-se até 200 transferências de subárvore, e que esta razão cai para 1,5 à medida que o número de transferências de subárvore utilizadas na geração das instâncias diminui. Este algoritmo pode ser estendido para tratar instâncias do problema da floresta de concordância ótima com árvores cujos nós tenham grau no máximo $d \geq 2$; no presente trabalho descrevemos e analisamos um algoritmo de $(d+1)$-aproximação para este problema. Finalmente, desenvolvemos uma prova, baseada em Hein [19, 20], de que o problema da floresta de concordância ótima para árvores com grau no máximo 2 é APX-difícil. Esse resultado mostra que, sob a hipótese de que $\mathrm{P} \neq \mathrm{NP}$, não existe um esquema de aproximação polinomial para o problema. Ou seja, não existe para todo $\epsilon>0$ um algoritmo de aproximação com razão $1+\epsilon$. Este resultado, junto com a $(d+1)$-aproximação para o problema da floresta de concordância ótima para árvores com grau no máximo $d$ que provamos, mostra que este último problema é APX-completo e que o problema da floresta de concordância ótima para árvores sem restrição nos graus dos nós é APX-difícil. 
Os Capítulos 1 e 2 apresentam a notação e os conceitos básicos sobre Teoria dos Grafos e Análise de Algoritmos que serão utilizados neste trabalho.

O Capítulo 3 introduz os conceitos necessários para o estudo do problema da floresta de concordância ótima.

O Capítulo 4 apresenta os resultados de aproximabilidade obtidos para este problema com árvores de grau no máximo 2. Neste capítulo são descritos e analisados o algoritmo de Hein e dois dos algoritmos de 3-aproximação propostos neste trabalho para o problema.

O Capítulo 5 descreve as implementações dos algoritmos descritos no Capítulo 4 e apresenta o terceiro algoritmo de 3-aproximação que propomos para o problema da floresta de concordância ótima. Este algoritmo foi projetado para fornecer bons limitantes inferiores para o valor da solução ótima das instâncias do problema, e por esta razão é apresentado neste capítulo, enquanto os demais algoritmos de 3aproximação são apresentados no Capítulo 4 .

O Capítulo 6 apresenta e analisa um algoritmo de $(d+1)$-aproximação para o problema da floresta de concordância ótima para árvores de grau no máximo $d$.

O Capítulo 7 apresenta a prova de que o problema da floresta de concordância ótima para árvores com grau no máximo 2 é APX-difícil.

\section{Transferências de subárvore e eventos de recombinação}

Os Capítulos 3 a 7 apresentam e testam algoritmos de aproximação e provam resultados de inaproximabilidade para o problema da floresta de concordância ótima. A relação entre eventos de recombinação, operações de transferência de subárvore e florestas de concordância é tratada nos dois últimos capítulos.

O Capítulo 8 define um conjunto canônico de transferências de subárvore, cujas operações são capazes de simular todas as transferências de subárvore possíveis em uma árvore filogenética com nós de grau no máximo 2. O principal resultado deste capítulo é a equivalência de duas noções de distância entre árvores filogenéticas: a distância dada pelo número mínimo de transferências de subárvore canônicas que transformam uma das árvores na outra, e a distância dada pelo número de componentes de uma floresta de concordância ótima das duas árvores. Descrevemos um algoritmo que transforma uma floresta de concordância de tamanho $m$ de duas árvores filogenéticas em uma seqüência de $m-1$ transferências canônicas que transforma uma árvore na outra.

O Capítulo 9 discute a relação entre transferências de subárvore e o modelo de Hein para a análise de dados sequenciais pré-alinhados sujeitos a recombinação. Aqui, discutimos a aplicabilidade das transferências canônicas a este modelo, tomando como 
critério de aplicabilidade a correspondência um-a-um entre transferências canônicas e eventos de recombinação. Em nossa análise concluímos que uma das operações canônicas equivale a pelo menos dois eventos de recombinação. Com base nesse argumento, definimos um conjunto padrão de transferências de subárvore, contendo apenas as transferências de subárvore canônicas que equivalem a um único evento de recombinação, e introduzimos o conceito correspondente de distância padrão entre árvores filogenéticas. O resultado central deste capítulo é que a distância canônica e a distância padrão entre árvores filogenéticas diferem de no máximo uma unidade. Este resultado, mais os resultados do Capítulo 8, implicam que a distância padrão de transferência de subárvore e o tamanho de uma floresta de concordância ótima de duas árvores também diferem de no máximo uma unidade. Como no Capítulo 8, descrevemos um algoritmo que converte uma floresta de concordância de duas árvores filogenéticas de tamanho $m$ em uma seqüência de transferências padrão de subárvore com no máximo $m$ operações. Os resultados de aproximabilidade que obtemos como conseqüência desses resultados são os seguintes: os problemas de determinar a distância canônica e a distância padrão entre duas árvores filogenéticas são 3-aproximáveis, e o problema de determinar a distância canônica é APX-completo. 
Parte I

\section{Preliminares}




\section{Capítulo 1}

\section{Noções fundamentais}

O nosso propósito neste capítulo e no seguinte é estabelecer a notação e a terminologia que usaremos neste trabalho.

\subsection{Conjuntos e funções}

Os conjuntos dos números naturais, racionais e reais são denotados pelos símbolos $\mathbb{N}, \mathbb{Q}$ e $\mathbb{R}$, respectivamente. Para qualquer destes conjuntos, o subscrito * indica a exclusão do zero, o sobrescrito + indica a exclusão dos números negativos e o sobrescrito - indica a exclusão dos números positivos. Assim, por exemplo, o conjunto dos naturais não-negativos é indicado por $\mathbb{N}^{+}$e o conjunto dos racionais positivos é indicado por $\mathbb{Q}_{*}^{+}$.

Um conjunto $X$ com cardinalidade $k$ é chamado $k$-conjunto. Se $X$ é um subconjunto de cardinalidade $k$ de um conjunto $Y$, então $X$ é um $k$-subconjunto de $Y$.

Se $f: X \rightarrow Z$ é uma função, então denotamos a restrição da função $f$ a um conjunto $Y \subseteq X$ por $\left.f\right|_{Y}$ e a imagem de $\left.f\right|_{Y}$ por $f(Y)$.

Usamos o termo mapeamento como sinônimo de função bijetora.

Reservamos o termo família para designar subconjuntos do conjunto das partes de um conjunto a que estejamos nos referindo em um determinado contexto. Se $\mathcal{X}$ é uma família de subconjuntos de um conjunto finito, então denotamos o conjunto $\bigcup_{X \in \mathcal{X}} X$ por $\bigcup \mathcal{X}$

Se $X$ é um conjunto, então uma família de subconjuntos de $X$ dois a dois disjuntos é um empacotamento de $X$, e uma família $\mathcal{X}$ de subconjuntos de $X$ tal que todo elemento de $X$ pertence a $\bigcup \mathcal{X}$ é uma cobertura de $X$. Se cada elemento de $X$ pertence a exatamente um conjunto em $\mathcal{X}$, então $\mathcal{X}$ é uma cobertura exata de $X$. 
Se $c$ é um vetor de números reais indexado por um conjunto finito $X$, e $Y \subseteq X$, então denotamos $\sum_{y \in Y} c(y)$ por $c(Y)$.

Usamos a notação := para expressar que um determinado símbolo (o que está do lado esquerdo de $:=$ ) denota a expressão que está do lado direito de $:=$.

\subsection{Grafos}

Os conceitos e a notação apresentados a seguir são baseados em textos clássicos sobre grafos, como Bondy e Murty [6] e Berge [4].

Um grafo (não-orientado) $H$ é uma tripla ordenada $\left(V(H), A(H), \Psi_{H}\right)$, onde $V(H)$ é um conjunto finito não-vazio de elementos que chamamos de vértices, $\boldsymbol{A}(\boldsymbol{H})$ é um conjunto finito disjunto de $V(H)$ cujos elementos são chamados de arestas, e $\Psi_{H}$ é uma função injetora, chamada função de incidência, que associa a cada aresta de $H$ um par não-ordenado de vértices distintos. Observe que esta definição exclui a possibilidade de ocorrerem "laços" e "arestas múltiplas", segundo a definição dada em Bondy e Murty [6] para estes dois elementos.

A ordem de um grafo é o número de vértices do mesmo. Em particular, grafos de ordem um são chamados triviais.

Se $x, y$ são vértices de um grafo $H$, e $e$ é uma aresta de $H$ tal que $\Psi_{H}(e)=\{x, y\}$, então dizemos que os vértices $x$ e $y$ são as extremidades da aresta $e$. Também dizemos que a aresta $e$ incide em $x$ e $y$, e que os vértices $x$ e $y$ são adjacentes em $H$. Duas arestas com uma extremidade em comum são ditas adjacentes. Uma aresta pode ser indicada pelas notações $x y$ ou $y x$ se $x$ e $y$ são as suas extremidades.

O número de vértices adjacentes a um vértice $x$ de um grafo $H$ é o grau de $x$ em $H$. Vértices de grau zero em $H$ são ditos isolados.

Se $H$ e $H^{\prime}$ são dois grafos então dizemos que $H^{\prime}$ é um subgrafo de $H$ se $V\left(H^{\prime}\right) \subseteq$ $V(H), A\left(H^{\prime}\right) \subseteq A(H)$, e $\Psi_{H^{\prime}}=\left.\Psi_{H}\right|_{A\left(H^{\prime}\right)}$. Um subgrafo de $H$ diferente de $H$ é um subgrafo próprio de $H$. Se $H$ e $H^{\prime}$ são dois grafos tais que $V(H) \cap V\left(H^{\prime}\right)=\emptyset$, então $H$ e $H^{\prime}$ são disjuntos nos vértices. Se $A(H) \cap A\left(H^{\prime}\right)=\emptyset$, então $H$ e $H^{\prime}$ são disjuntos nas arestas.

Dois grafos $H$ e $H^{\prime}$ são isomorfos se existem mapeamentos de $V(H)$ em $V\left(H^{\prime}\right)$ e de $A(H)$ em $A\left(H^{\prime}\right)$ que preservam a função de incidência entre os vértices. Neste caso, o mapeamento é denominado um isomorfismo entre $H$ e $H^{\prime}$.

Em um grafo $H$, uma seqüência da forma

$$
\left(x_{0}, e_{0}, \ldots, x_{p-1}, e_{p-1}, x_{p}\right), \quad p \geq 0,
$$

é um passeio em $H$ se $x_{i} \in V(H)$ para $i=0, \ldots, p ; e_{i} \in A(H)$ para $i=0, \ldots, p-1$; e $\Psi_{H}\left(e_{i}\right)=\left\{x_{i}, x_{i+1}\right\}$ para $i=0, \ldots, p-1$. Os vértices $x_{0}$ e $x_{p}$ são as extremidades 
do passeio, e $p$ é o comprimento do passeio. Dizemos que um passeio conecta as suas extremidades. Um passeio $\left(x_{0}, e_{0}, \ldots, x_{p-1}, e_{p-1}, x_{p}\right)$ é um caminho se $x_{i} \neq x_{j}$ para $i$ e $j$ tais que $0 \leq i<j \leq p$, e um circuito se $p \geq 1, x_{i} \neq x_{j}$ para $i$ e $j$ tais que $0 \leq i<j<p$, e $x_{p}=x_{0}$. Um circuito em um grafo que contém todos os seus vértices é um circuito hamiltoniano.

Considere a relação de equivalência sobre os vértices de um grafo $H$ definida da seguinte maneira: dois vértices de $H$ são equivalentes se existe um caminho entre eles. Os subgrafos de $H$ induzidos pelas classes de equivalência desta relação são os componentes de $H$. Se $H$ tem um único componente, então $H$ é um grafo conexo, caso contrário $H$ é um grafo não-conexo.

A distância entre dois vértices de um grafo $H$ é o comprimento de um caminho de comprimento mínimo entre estes vértices. Se os dois vértices não pertencem a um mesmo componente de $H$, então por convenção a distância entre eles é $+\infty$.

Uma floresta é um grafo que não possui circuitos. Uma floresta conexa é chamada árvore. Uma floresta de $H$ é um subgrafo de $H$ que é uma floresta. Pode-se provar facilmente as seguintes caracterizações para uma árvore $H$ : (a) $H$ é conexo e $|A(H)|=$ $|V(H)|-1$; e (b) para cada par de vértices $x$ e $y$ de $H$ existe exatamente um caminho em $H$ com extremidades $x$ e $y$.

Um grafo orientado $D$ é uma tripla ordenada $\left(V(D), A(D), \Psi_{D}\right)$ onde $V(D)$ é um conjunto finito não-vazio de elementos chamados de nós, $A(D)$ é um conjunto finito disjunto de $V(D)$ cujos elementos são chamados de $\operatorname{arcos,~e~} \Psi_{D}$ é uma função injetora, chamada função de incidência, que associa a cada arco de $D$ um par ordenado de nós distintos. Como no caso dos grafos não-orientados, aqui também estão excluídas as ocorrências de "laços" e "arcos múltiplos".

A cada grafo $D$ podemos associar um grafo não-orientado $H$, chamado grafo subjacente de $D$, obtido de $D$ substituindo-se cada arco de $D$ por uma aresta com as mesmas extremidades. Se dois arcos de $D$ têm as mesmas extremidades, então apenas um deles é substituído, de modo que o grafo subjacente não admita "arestas múltiplas". Também podemos associar um grafo orientado $D$ a um grafo não-orientado $H$, fazendo $V(D):=V(H), A(D):=A(H)$ e $\Psi_{D}(e):=(x, y)$ ou $\Psi_{D}(e):=(y, x)$ para cada $e \in A(H)$ tal que $\Psi_{H}(e)=\{x, y\}$; neste caso, dizemos que $D$ é uma orientação de $H$. Vários dentre os conceitos definidos para grafos não-orientados aplicam-se a grafos orientados através dos grafos subjacentes destes últimos. É o caso de conceitos como grafos triviais, incidência, adjacência, grau, isomorfismo, passeios, caminhos, componentes e distância. Mas há também conceitos exclusivos de grafos orientados, que envolvem a noção de orientação de arcos.

Se $x, y$ são nós de um grafo orientado $D$, e $e$ é um arco de $D$ tal que $\Psi_{D}(e)=(x, y)$, então dizemos que o arco $e$ chega em $y$ e sai de $x$. Um arco pode ser indicado pela notação $x y$ se ele sai de $x$ e chega em $y$. 
Se $D$ e $D^{\prime}$ são dois grafos orientados tais que $V(D) \cap V\left(D^{\prime}\right)=\emptyset$, então $D$ e $D^{\prime}$ são disjuntos nos nós. Se $A(D) \cap A\left(D^{\prime}\right)=\emptyset$, então $D$ e $D^{\prime}$ são disjuntos nos arcos.

Em um grafo orientado $D$, uma seqüência da forma

$$
\left(x_{0}, e_{0}, \ldots, x_{p-1}, e_{p-1}, x_{p}\right), \quad p \geq 0,
$$

é um passeio se $x_{i} \in V(D)$ para $i=0, \ldots, p$; $e_{i} \in A(D)$ para $i=0, \ldots, p-1$; e $\Psi_{D}\left(e_{i}\right)=\left(x_{i}, x_{i+1}\right)$ ou $\Psi_{D}\left(e_{i}\right)=\left(x_{i+1}, x_{i}\right)$ para $i=0, \ldots, p-1$. Os vértices $x_{0} \mathrm{e}$ $x_{p}$ são as extremidades do passeio, e $p$ é o comprimento do passeio. Um passeio $\left(x_{0}, e_{0}, \ldots, x_{p-1}, e_{p-1}, x_{p}\right)$ é um caminho em $D$ se $x_{i} \neq x_{j}$ para $i$ e $j$ tais que $0 \leq i<$ $j \leq p$, e um circuito em $D$ se $p \geq 1, x_{i} \neq x_{j}$ para $i$ e $j$ tais que $0 \leq i<j<p$, e $x_{p}=x_{0}$.

Em um grafo orientado $D$, um passeio

$$
\left(x_{0}, e_{0}, \ldots, x_{p-1}, e_{p-1}, x_{p}\right), \quad p \geq 0,
$$

é orientado se $\Psi_{D}\left(e_{i}\right)=\left(x_{i}, x_{i+1}\right)$ para $i=0, \ldots, p-1$. Um passeio orientado $\left(x_{0}, e_{0}, \ldots, x_{p-1}, e_{p-1}, x_{p}\right)$ é um caminho orientado em $D$ se $x_{i} \neq x_{j}$ para $i$ e $j$ tais que $0 \leq i<j \leq p$, e um circuito orientado em $D$ se $p \geq 1, x_{i} \neq x_{j}$ para $i$ e $j$ tais que $0 \leq i<j<p$, e $x_{p}=x_{0}$. Grafos orientados sem circuitos orientados são chamados acíclicos.

\section{3 Árvores e florestas enraizadas}

Esta seção baseia-se em Bondy e Murty [6], Berge [4], Knuth [26] e Cormen et al. [12].

Uma floresta enraizada $G$ é uma floresta orientada em que cada componente $W$ de $G$ tem um nó especial, chamado de raiz de $W$ e denotado por $r_{W}$, tal que, para todo arco $x y$ de $W$, a extremidade $y$ está mais próxima de $r_{W}$ do que a extremidade $x$. Uma árvore enraizada é uma floresta enraizada conexa. Uma árvore enraizada conexa composta de um único nó (que é a sua raiz) é dita trivial. (Nas figuras não indicaremos a orientação dos arcos, devendo ficar subentendido que a orientação é sempre de baixo para cima.)

Para cada arco $e:=x y$ em uma floresta enraizada $G$, o nó $y$ é a extremidade superior de $e$, e o nó $x$ é a extremidade inferior de $e$. Indicamos a extremidade inferior do arco $e$ como $l_{G}(e)$ e a extremidade superior como $u_{G}(e)$. Se $x$ é um nó de uma floresta enraizada $G$ que não é a raiz de nenhum componente de $G$, então denotamos por $s_{G}(x)$ o arco cuja extremidade inferior é $x$. Vamos omitir os subscritos quando eles forem claros pelo contexto.

Se $x$ e $y$ são dois nós em uma floresta enraizada $G$ pertencentes a um mesmo componente $W$ de $G$, e $y$ está no caminho orientado que conecta $x$ a $r_{W}$, então $y$ é 
um nó antepassado de $x$, e $x$ é um nó descendente de $y$. Se $x$ e $y$ são distintos, então $y$ é um nó antepassado estrito de $x$, e $x$ é um nó descendente estrito de $y$. Note que, de acordo com esta definição, excetuando as raízes, todos os nós de uma floresta enraizada admitem um antepassado estrito. O antepassado estrito de $x$ cuja distância a $x$ é mínima é o antepassado imediato de $x$, também chamado pai de $x$. Se $y$ é o antepassado imediato de $x$, então $x$ é um descendente imediato de $y$, ou filho de $y$. Filhos distintos de um mesmo nó pai são chamados de nós irmãos. O grau de um nó é o número de descendentes imediatos deste nó. Nós com grau zero são chamados de folhas, e nós com grau diferente de zero são chamados de nós internos. O conjunto das folhas de $G$ é denotado por $L_{G}$. Se $y$ é um nó de $G$, então o conjunto das folhas de $G$ descendentes de $y$ é denotado por $L_{G}(y)$.

$\mathrm{O}$ antepassado comum mais recente (lowest common ancestor) de um conjunto de nós $x_{1}, \ldots, x_{m}, m \geq 1$, de uma árvore enraizada $W$ é o nó de $W$ mais distante da raiz que é um antepassado de todos esses nós $x_{i}, 1 \leq i \leq m$. Denotamos este nó por lca $W\left(\left\{x_{1}, \ldots, x_{m}\right\}\right)$, ou lca ${ }_{W}\left(x_{1}, \ldots, x_{m}\right)$, ou simplesmente lca $\left(x_{1}, \ldots, x_{m}\right)$. Pode-se provar que, se $x, y$ e $z$ são nós de $W$, então:

- $\operatorname{lca}(x, y, z)=\operatorname{lca}(x, \operatorname{lca}(y, z))=\operatorname{lca}(\operatorname{lca}(x, y), z)$.

- Se $v:=\operatorname{lca}(x, z)=\operatorname{lca}(y, z)$, então $\operatorname{lca}(x, y)$ é um descendente de $v$. Em árvores com grau máximo 2 , lca $(x, y)$ é sempre um descendente estrito de $v$.

Sejam $G$ e $G^{\prime}$ duas florestas enraizadas disjuntas nos vértices. A operação de união de $G$ e $G^{\prime}$, denotada por $G+G^{\prime}$, resulta no grafo com conjunto de vértices $V(G) \cup V\left(G^{\prime}\right)$, conjunto de arestas $A(G) \cup A\left(G^{\prime}\right)$, e para todo $e \in A(G) \cup A\left(G^{\prime}\right)$, $\Psi_{G+G^{\prime}}(e)=\Psi_{G}(e)$ se $e \in A(G)$, caso contrário $\Psi_{G+G^{\prime}}(e)=\Psi_{G^{\prime}}(e)$.

Seja $G$ uma floresta enraizada, e $e:=x y$ um arco de um componente $W$ de $G$. A operação de remoção do arco $e$ resulta na floresta enraizada $(V(G), A(G) \backslash$ $\left.\{e\},\left.\Psi_{G}\right|_{A(G) \backslash\{e\}}\right)$, sendo que $x$ passa a ser a raiz do novo componente que contém $x$ e $r_{W}$ passa a ser a raiz do novo componente que contém $y$. A remoção de em $G$ é denotada por $\operatorname{Rem}(G, e)$.

Seja $G$ uma floresta enraizada e $W$ um componente de $G$. Seja $x$ um nó de $G$ não contido em $W$, e $V$ o componente de $G$ que contém $x$. A operação de conexão da árvore $W$ na floresta $G$ através do nó $x$ produz a floresta enraizada obtida acrescentando-se a $\mathcal{G}$ o arco $r_{W} x$, sendo que $r_{V}$ passa a ser a raiz do novo componente produzido pela operação. Esta operação é indicada pela notação $\operatorname{Cnx}(W, G, x)$.

Seja $G$ uma floresta enraizada, e $e:=x y$ um arco de $G$ tal que $y$ tem grau $1 \mathrm{em}$ $G$. A operação de contração do arco $x y$ consiste de duas etapas. Na primeira etapa, é produzida a floresta enraizada obtida de $G$ pela operação $\operatorname{Rem}(G, e)$, e na segunda etapa identifica-se $x$ e $y .{ }^{1}$ Veja a Figura 1.2 .

\footnotetext{
${ }^{1}$ Esta operação é chamada algumas vezes na literatura $([19,20,1])$ de contração forçada.
} 
Seja $G$ uma floresta enraizada, $v$ um nó de $G$, e $W$ o componente de $G$ que contém $v$. A operação de extensão do nó $v$ também consiste de duas etapas. A primeira etapa produz a floresta enraizada obtida de $G$ pela substituição de $v$ por dois novos nós $x$ e $y$ tais que os arcos que entram em $v$ passam a entrar em $x$ e o arco que sai de $v$ passa a sair de $y$. Na segunda etapa é acrescentado o arco $x y$. A raiz do novo componente assim obtido passa a ser $r_{W}$ se $v \neq r_{W}$, caso contrário passa a ser $y$. Veja a Figura 1.1. 

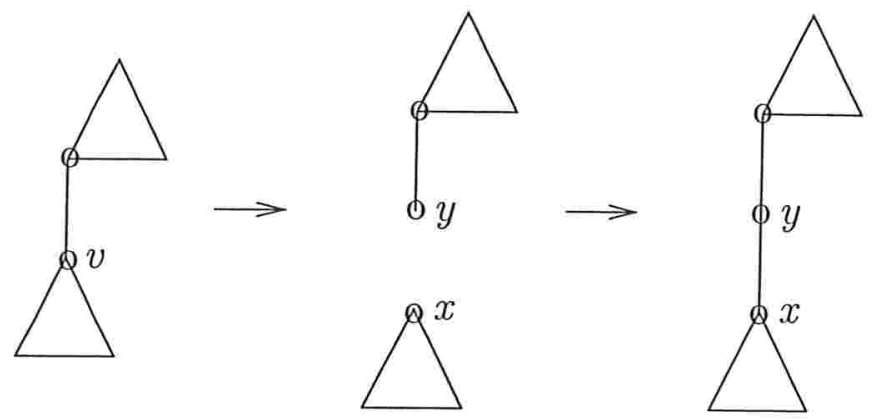

extensão

( $v$ não é raiz)

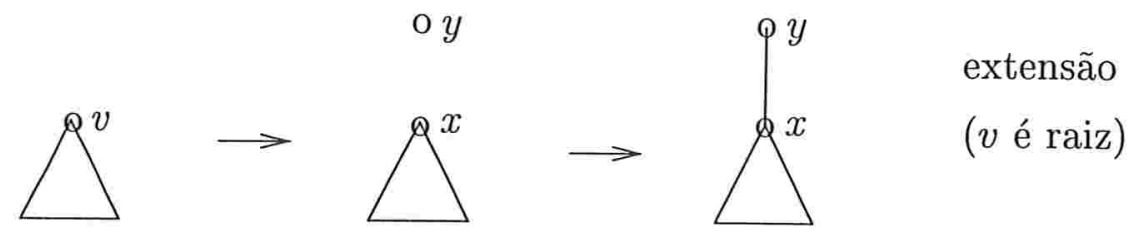

Figura 1.1: Extensão em árvores enraizadas.
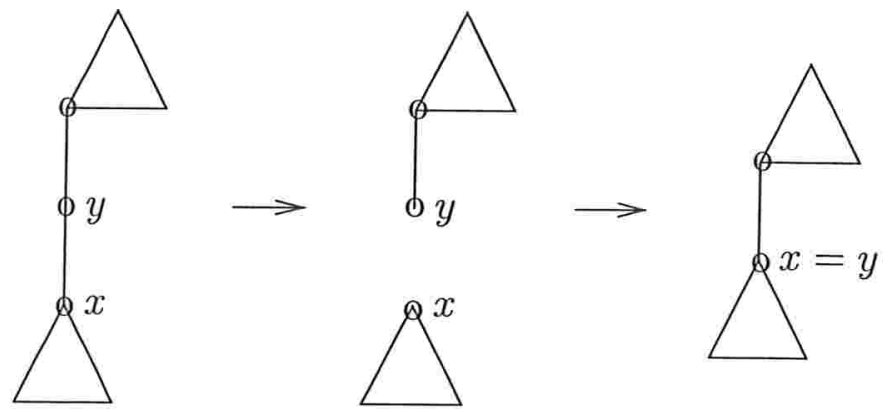

contração de $x y$ ( $y$ não é raiz)<smiles>[Ge]=C1CC1</smiles>
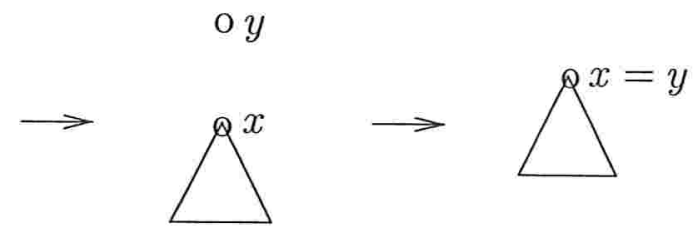

contração de $x y$ ( $y$ é raiz)

Figura 1.2: Contração em árvores enraizadas. 


\section{Capítulo 2}

\section{Classes de problemas, algoritmos e complexidade}

O sumário sobre problemas, algoritmos e complexidade apresentados neste capítulo é baseado em Fernandes et al. [15], Ausiello et al. [3], Papadimitriou [30], Garey e Johnson [16] e Cormen et al. [12]. O nosso propósito aqui é estabelecer a notação e a terminologia que usaremos neste trabalho.

\subsection{Conceitos básicos}

O conceito de problema pode ser formalizado como o de uma entidade formada por três componentes: um conjunto de objetos matemáticos chamados de instâncias, um conjunto de soluções para cada instância, e uma questão proposta a respeito destas instâncias. Resolver uma instância do problema é dar uma solução daquela instância que satisfaça a questão. Resolver um problema é resolver todas as instâncias do problema.

Há uma série de bons motivos para que se queira comparar problemas quanto ao grau de dificuldade para se resolvê-los. Para isso, necessitamos em primeiro lugar de um modelo de computação, ou seja, um dispositivo computacional abstrato capaz de executar certas instruções sobre dados que lhe são fornecidos, produzindo outros dados a partir deles. O modelo RAM (Random Access Machine), que vamos utilizar em nosso trabalho, é um dos modelos mais comuns em Ciência da Computação. Este modelo é composto de um número finito de posições de memória, uma unidade de processamento que executa instruções sequencialmente, e dispositivos de entrada e saída. Vamos assumir que todo número inteiro que aparece nos problemas de que vamos tratar pode ser armazenado em qualquer das posições de memória do dispositivo computacional. O conjunto de instruções de nosso modelo RAM é o de 
um pseudocódigo semelhante a uma linguagem de alto nível como Pascal.

Definido o modelo de computação e dado um problema, podemos escrever seqüências finitas de instruções, chamadas de algoritmos, que, ao serem lidas e executadas pelo dispositivo abstrato dado pelo modelo, sejam capazes de resolver o problema em questão.

Um algoritmo tipicamente lê uma entrada, ou seja, uma estrutura de dados contendo a instância do problema a ser resolvida, e escreve uma saída, isto é, uma outra estrutura de dados contendo uma solução da instância que satisfaça a questão. Neste texto, entradas e saídas de algoritmos são descritas como entidades matemáticas abstratas; as estruturas de dados são empregadas diretamente apenas nas análises. O espaço ocupado por estas estruturas de dados será usado para indicar o tamanho das entradas e saídas, que indicaremos entre barras $(|\cdot|)$. Vamos indicar a saída de um algoritmo $A$ para uma entrada $x$ como $A(x)$.

Dizemos que um algoritmo é correto para um problema, ou que ele resolve o problema, se, para cada instância deste problema, ele pára e sua saída corresponde a uma solução da instância que satisfaz a questão.

Um tema de grande interesse em Ciência da Computação é o desenvolvimento e a análise de algoritmos para resolver problemas. Neste contexto, surge naturalmente a questão da existência de algoritmos eficientes, ou seja, que consomem apenas uma porção razoável dos recursos computacionais disponibilizados pelo modelo de computação em função do tamanho da entrada. Esta noção induz naturalmente o conceito de problemas tratáveis e intratáveis (dependendo se existem ou não algoritmos eficientes que os resolvam). Estas questões, pioneiramente discutidas por Cobham [10] e Edmonds [14], são formalmente tratadas através da noção de complexidade de algoritmos e de problemas.

Neste texto, consideramos dois tipos de recursos computacionais: tempo e espaço de computação. Entende-se por complexidade de tempo de um algoritmo o número máximo de instruções requeridas pelo algoritmo sobre todas as entradas possíveis, e por complexidade de espaço de um algoritmo o número máximo, sobre todas as entradas possíveis, de símbolos escritos pelo algoritmo como "rascunho" durante a sua execução ${ }^{1}$. A complexidade de tempo (espaço) de um problema é a complexidade de tempo (espaço) mínima necessária para resolver o problema usando algum algoritmo. Estas complexidades são em geral escritas como funções no tamanho da entrada, geralmente expressas através da notação $O$ (Cormen et al. [12]).

O critério de eficiência de um algoritmo e tratabilidade de um problema mais aceito é o de que todas as complexidades acima sejam polinomialmente limitadas no tamanho da entrada. Dizemos que uma função $f: \mathbb{N} \rightarrow \mathbb{N}$ é polinomialmente limitada em $n$ se $f(n)=O\left(n^{k}\right)$ para algum $k \geq 1$. Algoritmos com complexidade de

\footnotetext{
${ }^{1} \mathrm{O}$ tamanho da entrada e da saída não é computado na definição de complexidade de espaço.
} 
tempo polinomialmente limitado no tamanho da entrada são chamados polinomiais. Neste trabalho estamos interessados em classificar problemas e algoritmos de acordo com este critério.

\subsection{Problemas de decisão}

Um dos tipos de problemas que exerceram e exercem um papel importante na teoria da complexidade são os problemas de decisão. Um problema de decisão é um problema em que cada instância admite apenas uma solução, que pode ser "SIM" ou "NÃO". A teoria da complexidade desenvolveu-se em torno de problemas de decisão, por dois motivos. Primeiro, eles podem ser facilmente modelados como problemas de linguagens sobre um alfabeto finito, de modo que a complexidade de se resolvê-los corresponde à complexidade de se decidir suas linguagens correspondentes. E segundo, outros tipos de problemas mais complexos podem ser facilmente convertidos em problemas de decisão com complexidades equivalentes, conforme veremos mais adiante.

Um problema de decisão $\Lambda$ é um par $\left(I_{\Lambda}, Y_{\Lambda}\right)$, onde $I_{\Lambda}$ é o conjunto das instâncias de $\Lambda$, e $Y_{\Lambda} \subseteq I_{\Lambda}$ é o conjunto das instâncias positivas de $\Lambda$. "SIM".

Em geral convencionamos que as instâncias positivas são aquelas cuja solução é

Os problemas de decisão tratáveis - aqueles que podem ser resolvidos por algoritmos polinomiais - são agrupados numa classe de problemas chamada $\mathbf{P}$. Nesta classe há problemas práticos importantes, como por exemplo o problema de programação linear. Entretanto, vários problemas igualmente importantes não puderam até o momento ser incorporados a esta classe, como por exemplo o problema clássico de se decidir se um grafo tem um circuito hamiltoniano.

Uma fração significativa destes problemas, no entanto, tem uma outra característica que foi observada cedo no desenvolvimento da teoria da complexidade: suas instâncias positivas admitem certificados sucintos de que elas são de fato positivas. Este é o caso do problema do circuito hamiltoniano, em que uma instância positiva pode ser verificada apresentando-se um circuito hamiltoniano desta instância. A classe que agrupa estes problemas é denominada de NP (Non-deterministic Polynomial time).

Um problema de decisão $\Lambda$ pertence à classe de problemas NP se:

- existe um problema de decisão $\Lambda^{\prime}$ em $\mathrm{P}$ cujas instâncias são pares ordenados da forma $(x, y)$, tal que

$$
Y_{\Lambda}=\left\{x:(x, y) \in Y_{\Lambda^{\prime}} \text { para alguma cadeia de caracteres } y\right\} ;
$$


- se $(x, y) \in Y_{\Lambda^{\prime}}$ então $|y| \leq|x|^{k}$ para algum $k \geq 1$.

Não se sabe se as classes P e NP coincidem ou se elas são distintas; de fato, esta é a questão em aberto mais importante da teoria da complexidade. Sabe-se apenas que $\mathrm{P} \subseteq \mathrm{NP}$, e acredita-se que estes conjuntos sejam distintos.

Uma forma de estudar a estrutura dos problemas em NP na tentativa de caracterizar porque eles aparentemente são mais difíceis de serem resolvidos em tempo polinomial do que aqueles em $\mathrm{P}$, é definir o que seriam os problemas mais difíceis (no sentido da existência de um algoritmo polinomial) em NP. Para isso, usamos os conceitos de oráculo e Karp-redução entre problemas.

Um oráculo para um problema é um dispositivo computacional abstrato que recebe como entrada uma instância do problema e escreve na saída, em uma unidade de tempo, uma solução da instância dada que satisfaça a questão.

Uma Karp-redução de um problema de decisão $\Lambda$ a um problema de decisão $\Lambda^{\prime}$ é um algoritmo polinomial $A$ que aceita como entrada uma instância $x$ de $\Lambda$ e devolve $A(x)$, tal que $A(x)$ é uma instância de $\Lambda^{\prime}$ e $x \in Y_{\Lambda} \Longleftrightarrow A(x) \in Y_{\Lambda^{\prime}}$. Dizemos que $\Lambda$ é Karp-redutível a $\Lambda^{\prime}$.

Um problema de decisão $\Lambda$ é dito NP-completo se $\Lambda \in \mathrm{NP}$ e todo problema $\Lambda^{\prime} \in$ NP é Karp-redutível a $\Lambda$.

Os problemas NP-completos podem ser considerados como os mais difíceis no conjunto NP, já que, como conseqüência da definição acima, se fosse conhecido um algoritmo polinomial que resolvesse um problema NP-completo, então todos os problemas em NP teriam complexidade de tempo polinomial, e portanto teríamos provado que $\mathrm{P}=\mathrm{NP}$. A primeira prova de NP-completude para um problema de decisão foi apresentada em 1971 por Cook [11] para o problema da satisfatibilidade; em seguida, Karp [23] apresentou uma coleção de resultados provando que vários problemas de decisão são tão difíceis quanto o problema da satisfatibilidade. Desde então demonstrou-se que muitos outros problemas são NP-completos. Garey e Johnson [16] e Ausiello et al. [3] trazem extensas coletâneas de problemas NP-completos.

Uma propriedade muito importante da Karp-redução é a transitividade: se temos três problemas de decisão $\Lambda, \Lambda^{\prime}$ e $\Lambda^{\prime \prime}$ tais que $\Lambda$ é Karp-redutível a $\Lambda^{\prime}$, e $\Lambda^{\prime}$ é Karpredutível a $\Lambda^{\prime \prime}$, então $\Lambda$ é Karp-redutível a $\Lambda^{\prime \prime}$. Uma conseqüência dessa propriedade é que podemos mostrar que um problema de decisão $\Lambda$ que está na classe NP é NPcompleto exibindo uma Karp-redução de um outro problema de decisão NP-completo a $\Lambda$. Dessa forma, as provas de NP-completude tornam-se muito mais simples. 


\subsection{Problemas de otimização}

Problemas de decisão têm um papel importante na teoria da complexidade. Os problemas encontrados na prática, no entanto, costumam aparecer sob formas mais gerais. Um exemplo são os problemas de otimização, em que tipicamente deseja-se saber o valor ótimo de uma função de medida dada pelo problema, junto com uma solução viável que otimize esta função.

Um problema de otimização $\Pi$ é uma quádrupla $\left(I_{\Pi}, \mathrm{SOL}_{\Pi}, m_{\Pi}, \mathrm{obj}_{\Pi}\right)$, onde:

- $I_{\Pi}$ é o conjunto das instâncias de $\Pi$;

- para $x \in I_{\Pi}, \mathrm{SOL}_{\Pi}(x)$ é o conjunto das soluções viáveis de $x$;

- para $x \in I_{\Pi}$ e $y \in \operatorname{SOL}_{\Pi}(x), m_{\Pi}(x, y) \in \mathbb{N}^{*}$ é a medida da solução viável $y$ para a instância $x$ de $\Pi$;

- $\operatorname{obj}_{\Pi} \in\{\min , \max \}$ define se o problema $\Pi$ é de minimização (min) ou de maximização $(\max )$.

Se $x$ é uma instância de um problema de otimização $\Pi$ tal que $\operatorname{SOL}_{\Pi}(x) \neq \emptyset$, então $x$ é uma instância viável de $\Pi$, caso contrário $x$ é uma instância inviável de $\Pi$.

Uma solução viável $y^{*}$ de uma instância $x$ de $\Pi$ é uma solução ótima de $x$ se $m_{\Pi}\left(x, y^{*}\right)=\operatorname{obj}_{\Pi}\left\{m_{\Pi}(x, y): y \in \operatorname{SOL}_{\Pi}(x)\right\}$. Denotamos o valor da solução ótima de $x$ (se existente) por $m_{\Pi}^{*}(x)$, e o conjunto das soluções ótimas de $x$ como $\operatorname{SOL}_{\Pi}^{*}(x)$.

Da mesma forma que procedemos com os problemas de decisão, também consideramos classes de problemas de otimização que podem ser resolvidas em tempo polinomial. Em primeiro lugar, definimos a classe dos problemas de otimização em que as soluções viáveis podem ser certificadas em tempo polinomial.

Um problema de otimização $\Pi$ pertence à classe de problemas NPO se satisfaz as seguintes condições:

- o problema de decidir se uma dada entrada é uma instância de П está em P;

- existe um problema de decisão $\Pi^{\prime} \in \mathrm{P}$ cujas instâncias são pares ordenados da forma $(x, y)$, e para $x \in I_{\Pi}$ temos que $\operatorname{SOL}_{\Pi}(x)=\left\{y:(x, y) \in Y_{\Pi^{\prime}}\right\}$;

- para todo $(x, y) \in Y_{\Pi^{\prime}}$ temos que $|y| \leq|x|^{k}$ para algum $k \geq 1$;

- $m_{\Pi}$ é polinomialmente computável.

Um problema de otimização $\Pi$ pertence à classe de problemas PO se $\Pi \in$ NPO e existe um algoritmo polinomial que, para cada instância $x$ de $\Pi$, devolve uma solução ótima $y \in \mathrm{SOL}_{\Pi}^{*}(x)$ e o valor $m_{\Pi}^{*}(x)$ da solução ótima. 
Pela definição acima, temos PO $\subseteq$ NPO, mas a exemplo do que ocorre com as classes de problemas P e NP, não sabemos se as classes PO e NPO coincidem. De fato, pode-se provar que elas são distintas a menos que P e NP coincidam.

A todo problema de otimização podemos associar um problema de decisão tal que o problema de otimização pode ser resolvido em tempo polinomial se dispusermos de um oráculo para o problema de decisão associado. Isso permite que aproveitemos os resultados de complexidade existentes para problemas de decisão para caracterizar a complexidade dos problemas de otimização.

Se $\Pi$ é um problema de otimização, então definimos $\Pi_{D}$, o problema de decisão associado a $\Pi$, da seguinte maneira:

$$
\begin{aligned}
& \text { - } I_{\Pi_{D}}:=\left\{(x, k): x \in I_{\Pi}, k \in \mathbb{N}^{*}\right\} \\
& \text { - } Y_{\Pi_{D}}:= \begin{cases}\left\{(x, k) \in I_{\Pi_{D}}: m_{\Pi}^{*}(x) \leq k\right\} & \text { se } \operatorname{obj}_{\Pi}=\min \\
\left\{(x, k) \in I_{\Pi_{D}}: m_{\Pi}^{*}(x) \geq k\right\} & \text { se } \operatorname{obj}_{\Pi}=\max \end{cases}
\end{aligned}
$$

O problema $\Pi_{D}$ é chamado versão decisão do problema $\Pi$.

Para estabelecer a relação entre as complexidades dos problemas de otimização e de seus problemas de decisão associados, vamos introduzir um tipo mais geral de transformação entre problemas.

Uma Turing-redução de um problema $\Pi$ para um problema $\Pi^{\prime}$ é um algoritmo polinomial $A$ que resolve $\Pi$ utilizando um oráculo para o problema $\Pi^{\prime}$. Se existe uma Turing-redução de $\Pi$ para $\Pi^{\prime}$, então dizemos que $\Pi$ é Turing-redutível a $\Pi^{\prime}$.

É possível mostrar que, se $\Pi$ é um problema de otimização em NPO, então $\Pi_{D}$ é Turing-redutível a $\Pi$, e se além disso $\Pi_{D}$ é NP-completo, então $\Pi$ é Turing-redutível a $\Pi_{D}$.

A seguinte definição caracteriza a complexidade dos problemas de otimização em NPO cujos problemas de decisão associados são NP-completos. Um problema de otimização $\Pi$ é NP-difícil se todo problema de decisão $\Pi^{\prime} \in N P$ é Turing-redutível a II.

Problemas NP-difíceis são tão difíceis quanto os problemas mais difíceis dentro de NP (isto é, os problemas NP-completos), e também são os problemas mais difíceis dentro de NPO, no seguinte sentido: se existisse um algoritmo polinomial que resolvesse um problema NP-difícil, então todos os problemas em NPO pertenceriam a PO e teríamos portanto $\mathrm{PO}=\mathrm{NPO}$ e $\mathrm{P}=\mathrm{NP}$.

Da mesma maneira que ocorre com as Karp-reduções, temos que as Turingreduções são transitivas. Uma conseqüência disso é que para se provar que um problema é NP-difícil pode-se empregar uma estratégia similar à que discutimos para resultados de NP-completude. Temos que, se II é um problema de otimização e $\Lambda$ 
é um problema de decisão NP-completo Turing-redutível a П, então П é NP-difícil. Em particular, se $\Pi_{D}$ é NP-completo então $\Pi$ é NP-difícil.

\subsection{Algoritmos de aproximação}

Conforme vimos nas seções anteriores, não se espera que existam algoritmos eficientes para resolver problemas de decisão em NP e problemas de otimização em NPO. O que se pode esperar, em termos de resoluções eficientes para estes tipos de problemas, é desenvolver algoritmos polinomiais que produzam soluções aproximadas, isto é, soluções viáveis que estejam "próximas" de uma solução ótima. Tais algoritmos são chamados algoritmos de aproximação.

Se $\Pi$ é um problema de otimização do tipo min, $x$ é uma instância de $\Pi$ e $y$ é uma solução viável de $x$, então a razão de desempenho ou razão de aproximação da solução $y$ é definida como

$$
R_{\Pi}(x, y):=\frac{m_{\Pi}(x, y)}{m_{\Pi}^{*}(x)} .
$$

Se П é um problema de otimização do tipo max, então a razão de desempenho da solução $y$ é definida como

$$
R_{\Pi}(x, y):=\frac{m_{\Pi}^{*}(x)}{m_{\Pi}(x, y)}
$$

Seja $A$ um algoritmo polinomial para um problema $\Pi$ em NPO. Se, para cada instância $x$ de $\Pi$ temos $R_{\Pi}(x, A(x)) \leq r$ para algum $r \geq 1$, então $A$ é um algoritmo de $r$-aproximação para o problema $\Pi$, ou uma $r$-aproximação para $\Pi$, e $\Pi$ é $r$ aproximável. A razão de aproximação ou razão de desempenho do algoritmo $A$ é definida como

$$
\inf \left\{r: R_{\Pi}(x, A(x)) \leq r \text { para toda instância } x \text { de } \Pi\right\} .
$$

A classe dos problemas em NPO que são $r$-aproximáveis para algum $r \geq 1$ é chamada APX.

Observe que as definições de razão de desempenho dadas acima implicam que tal razão é sempre maior ou igual a 1, sendo 1 para aqueles algoritmos que devolvem uma solução ótima para qualquer instância dada como entrada.

Pelo que vimos nas seções anteriores, não se espera conseguir algoritmos de aproximação com razão 1 para problemas de otimização NP-difíceis. Nestes casos, procurase desenvolver algoritmos de aproximação com razões o mais próximo possível de 1. A melhor maneira de conseguir isso é desenvolver algoritmos de aproximação parametrizados pela razão de desempenho, e que permitam que esta razão seja posicionada tão 
próxima de 1 quanto queiramos (geralmente, à custa de um acréscimo considerável na complexidade de tempo).

Seja $\Pi$ um problema em NPO. Um algoritmo $A$ é um esquema de aproximação polinomial para o problema $\Pi$ se $A$ admite como entradas uma instância de $\Pi$ e um racional, e se para cada racional $r>1$ e para cada instância x de $\Pi, A$ produz uma solução aproximada para $x$ com razão de desempenho no máximo $r$ em tempo polinomial em $|x|$. A classe dos problemas em NPO que admitem um esquema de aproximação polinomial é chamada de PTAS (Polynomial Time Approximation Scheme).

Temos $\mathrm{PO} \subseteq \mathrm{PTAS} \subseteq \mathrm{APX} \subseteq \mathrm{NPO}$, e pode-se provar que todas estas classes de problemas são distintas a menos que $\mathrm{P}=\mathrm{NP}$; além disso, conhecem-se problemas NP-difíceis que pertencem tanto a NPO \APX quanto a APX \PTAS. Isso mostra que as propriedades de aproximabilidade dos problemas em NPO e dos problemas NP-difíceis podem variar bastante entre si. Um estudo de problemas completos como o que foi feito para os problemas em NP, particularmente com relação às classes APX ou NPO, requer portanto uma noção de transformação entre problemas que preserve boas propriedades de aproximabilidade, para que problemas equivalentes (segundo a equivalência definida pela redução adotada) tenham as mesmas propriedades de aproximabilidade. A AP-redução, que apresentamos a seguir, possui esta característica, pois preserva a pertinência tanto em APX quanto em PTAS.

Sejam $\Pi_{1}$ e $\Pi_{2}$ dois problemas de otimização em NPO. Uma AP-redução de $\Pi_{1}$ a $\Pi_{2}$ é uma tripla ordenada $(A, B, \gamma)$ em que $A$ é um algoritmo que aceita como entradas uma instância de $\Pi_{1}$ e um racional $r$; $B$ é um algoritmo que aceita como entradas uma instância de $\Pi_{1}$, uma solução viável de uma instância de $\Pi_{2}$ e um racional $r$; e $\gamma \geq 1$ é um racional, tais que:

- para $x \in I_{\Pi_{1}}$ e racional $r>1$, temos que $A(x, r) \in I_{\Pi_{2}}$;

- para $x \in I_{\Pi_{1}}$, racional $r>1$ e $y \in \operatorname{SOL}_{\Pi_{2}}(A(x, r))$, temos que

$$
B(x, y, r) \in \operatorname{SOL}_{\Pi_{1}}(x)
$$

- para $x \in I_{\Pi_{1}}$, racional $r>1$ e $y \in \operatorname{SOL}_{\Pi_{2}}(A(x, r))$, temos que

$$
R_{\Pi_{2}}(A(x, r), y) \leq r \Rightarrow R_{\Pi_{1}}(x, B(x, y, r)) \leq 1+\gamma(r-1)
$$

- os algoritmos $A$ e $B$ têm complexidade de tempo polinomial para cada racional $r$.

Se existe uma AP-redução de um problema de otimização $\Pi_{1}$ a um problema de otimização $\Pi_{2}$, então dizemos que $\Pi_{1}$ é AP-redutível a $\Pi_{2}$. 
Um problema de otimização П em NPO é APX-difícil se todo problema em APX é AP-redutível a $\Pi$, e APX-completo se é APX-difícil e está em APX ${ }^{2}$. Observamos que, a exemplo das outras noções de redutibilidade que apresentamos, AP-reduções são transitivas; portanto podemos provar que um problema de otimização ПI é APXdifícil simplesmente exibindo uma AP-redução de um outro problema APX-difícil a $\Pi$.

De maneira análoga ao que ocorre com a classe NP, temos que problemas APXdifíceis não admitem esquemas de aproximação polinomial, a menos que $\mathrm{P}=\mathrm{NP}$, portanto uma prova de que um problema é APX-difícil indica que é pouco provável que esse problema admita um tal esquema.

Outra classe de problemas de otimização muito usada em resultados de inaproximabilidade é a classe MAX SNP. Problemas nesta classe caracterizam-se pela sua definição sintática, ao invés da sua complexidade, e problemas completos para esta classe são definidos com relação a um outro tipo de transformação entre problemas chamada L-redução. Provou-se que problemas nesta classe não admitem esquemas de aproximação polinomial a menos que P $=$ NP (Arora, Lund, Motwani, Sudan e Szegedy [2], ver Papadimitriou [30]), e, mais recentemente, que MAX SNP = APX quando definimos a classe MAX SNP usando um terceiro tipo de transformação entre problemas de otimização que preserva a aproximabilidade, diferente da AP-redução e da L-redução (Khanna, Motwani, Sudan e Vazirani [25], ver Ausiello et al. [3]). Damos a seguir a definição de L-redução, que é muito empregada na prática para provar-se que existem AP-reduções entre problemas de otimização, conforme veremos.

Sejam $\Pi_{1}$ e $\Pi_{2}$ dois problemas de otimização em NPO. Uma L-redução de $\Pi_{1}$ a $\Pi_{2}$ é uma quádrupla ordenada $(A, B, \gamma, \delta)$ em que $A$ é um algoritmo que aceita como entrada uma instância de $\Pi_{1}, B$ é um algoritmo que aceita como entradas uma instância de $\Pi_{1}$ e uma solução viável de uma instância de $\Pi_{2}$, e $\gamma$ e $\delta$ são dois racionais positivos tais que:

- para $x \in I_{\Pi_{1}}$, temos que $A(x) \in I_{\Pi_{2}}$;

- para $x \in I_{\Pi_{1}}$ e $y \in \mathrm{SOL}_{\Pi_{2}}(A(x))$, temos que $B(x, y) \in \operatorname{SOL}_{\Pi_{1}}(x)$;

- para $x \in I_{\Pi_{1}}$, temos que $m_{\Pi_{2}}^{*}(A(x)) \leq \gamma m_{\Pi_{1}}^{*}(x)$;

- para $x \in I_{\Pi_{1}}$ e $y \in \operatorname{SOL}_{\Pi_{2}}(A(x))$, temos que

$$
\left|m_{\Pi_{1}}^{*}(x)-m_{\Pi_{1}}(x, B(x, y))\right| \leq \delta\left|m_{\Pi_{2}}^{*}(A(x))-m_{\Pi_{2}}(A(x), y)\right|
$$

- os algoritmos $A$ e $B$ têm complexidade de tempo polinomial.

\footnotetext{
${ }^{2}$ Pode-se definir também problemas NPO-difíceis e NPO-completos, mas estas noções não serão empregadas neste trabalho.
} 
Se existe uma L-redução de um problema de otimização $\Pi_{1}$ a um problema de otimização $\Pi_{2}$, então dizemos que $\Pi_{1}$ é L-redutível a $\Pi_{2}$.

Uma propriedade importante das L-reduções é que, dados dois problemas de otimização $\Pi_{1} \in \mathrm{NPO}$ e $\Pi_{2} \in \mathrm{NPO}$, temos que, se $\Pi_{1} \in \mathrm{APX}$ e $\Pi_{1}$ é L-redutível a $\Pi_{2}$, então $\Pi_{1}$ é AP-redutível a $\Pi_{2}$. 


\section{Parte II}

\section{O problema da floresta filogenética de concordância ótima}




\section{Capítulo 3}

\section{Florestas filogenéticas de concordância}

Neste capítulo apresentamos as definições básicas sobre florestas de concordância e definimos os problemas envolvendo florestas de concordância que vamos abordar no presente trabalho.

\section{1 Árvores e florestas filogenéticas}

Uma floresta rotulada $\mathcal{G}$ é uma quádrupla ordenada $\left(T_{\mathcal{G}}, R_{\mathcal{G}}, S_{\mathcal{G}}, f_{\mathcal{G}}\right)$, onde:

- $T_{\mathcal{G}}$ é uma floresta enraizada;

- $\boldsymbol{R}_{\mathcal{G}}$ é o conjunto das raízes dos componentes de $T_{\mathcal{G}}$;

- $S_{\mathcal{G}}$ é um conjunto finito não-vazio;

- $f_{\mathcal{G}}$ é uma bijeção de $S_{\mathcal{G}}$ em $L_{\mathcal{G}}$, onde $L_{\mathcal{G}}$ é o conjunto das folhas de $\mathcal{G}$.

A floresta enraizada $T_{\mathcal{G}}$ é chamada de topologia da floresta rotulada $\mathcal{G}$ e o conjunto $S_{\mathcal{G}}$ é o conjunto dos rótulos das folhas de $\mathcal{G}$.

Se $u$ é um nó de uma floresta rotulada $\mathcal{G}$, então denotamos por $D(u)$ o conjunto dos rótulos das folhas de $\mathcal{G}$ que descendem de $u$. Se é um arco de $\mathcal{G}$, denotamos por $D_{e}$ o conjunto $D(l(e))$.

Uma árvore rotulada é uma floresta rotulada cuja topologia é conexa. A raiz de uma árvore rotulada é a raiz de sua topologia. Se $\mathcal{W}$ é uma árvore rotulada, então denotamos a sua raiz por $\boldsymbol{r}_{\mathcal{W}}$. Se $W$ é um componente da topologia $T_{\mathcal{G}}$ de uma floresta rotulada $\mathcal{G}$, então a árvore rotulada $\left(W,\left\{r_{W}\right\}, D\left(r_{W}\right),\left.f_{\mathcal{G}}\right|_{D\left(r_{W}\right)}\right)$ é um componente de $\mathcal{G}$. Uma árvore rotulada é trivial se sua topologia for trivial. Uma floresta rotulada é trivial se todos os seus componentes são triviais. 
Denotamos por $\mathcal{S}_{\mathcal{G}}$ a família dos conjuntos de rótulos dos componentes de $\mathcal{G}$, definida como:

$$
\mathcal{S}_{\mathcal{G}}:=\left\{D(r): r \in R_{\mathcal{G}}\right\}
$$

Note que $S_{\mathcal{G}}=\bigcup \mathcal{S}_{\mathcal{G}}$.

Uma floresta filogenética é uma floresta rotulada cuja topologia não admite nós com grau 1. Uma árvore filogenética é uma floresta filogenética cuja topologia é conexa.

\subsection{Restrições e projeções}

Se $\mathcal{G}$ e $\mathcal{H}$ são duas florestas rotuladas, então dizemos que $\mathcal{G}$ e $\mathcal{H}$ são isomorfas se:

- $T_{\mathcal{G}}$ e $T_{\mathcal{H}}$ são isomorfas;

- se o isomorfismo entre as topologias leva um componente $\mathcal{V}$ de $\mathcal{G}$ num componente $\mathcal{W}$ de $\mathcal{H}$, então este isomorfismo leva a raiz $r_{\mathcal{V}}$ na raiz $r_{\mathcal{W}}$;

- $S_{\mathcal{G}}=S_{\mathcal{H}}$

- para cada rótulo $a \in S_{\mathcal{G}}$, o isomorfismo leva $f_{\mathcal{G}}(a)$ em $f_{\mathcal{H}}(a)$.

Note que, se $\mathcal{G}$ e $\mathcal{H}$ são florestas isomorfas, então $\mathcal{S}_{\mathcal{G}}=\mathcal{S}_{\mathcal{H}}$.

Se $\mathcal{G}$ é uma floresta rotulada e $S$ é um subconjunto não-vazio dos rótulos de algum componente de $\mathcal{G}$, então:

- dizemos que $S$ define uma restrição simples em $\mathcal{G}$;

- a projeção simples de $S$ sobre $\mathcal{G}$, denotada por $\mathcal{G}[S]$, é a subárvore de $T_{\mathcal{G}}$ enraizada com conjunto de nós minimal que preserva os caminhos de cada nó de $f_{\mathcal{G}}(S)$ até o nó $\operatorname{lca}_{\mathcal{G}}\left(f_{\mathcal{G}}(S)\right)$;

- a restrição simples de $\mathcal{G}$ a $S$, denotada por $\mathcal{G} \mid S$, é a árvore rotulada com conjunto de nós minimal cuja topologia preserva os nós de $\mathcal{G}$ da forma lca $\left(f_{\mathcal{G}}\left(S^{\prime}\right)\right)$ para todo $S^{\prime} \subseteq S$, e que tem como raiz o nó $\operatorname{lca}_{\mathcal{G}}\left(f_{\mathcal{G}}(S)\right)$.

Observamos que $\mathcal{G}[S]$ é a subárvore enraizada de $T_{\mathcal{G}}$ com conjunto de nós minimal que preserva os nós de $\mathcal{G}$ da forma lca $\lg _{\mathcal{G}}\left(f_{\mathcal{G}}\left(S^{\prime}\right)\right)$ para todo $S^{\prime} \subseteq S$, e também os caminhos entre esses lcas. Observamos, ainda, que, se $\mathcal{G}$ é uma floresta filogenética, então os componentes de $\mathcal{G}$ são da forma $\mathcal{G} \mid S$ para cada $S \in \mathcal{S}_{\mathcal{G}}$.

A Figura 3.1 ilustra os conceitos de restrição simples e projeção simples. Podemos observar que cada nó de uma restrição simples corresponde a um nó da respectiva 
projeção simples, e cada arco de uma restrição simples corresponde a um caminho na projeção simples. Os nós intermediários de cada um destes caminhos, quando presentes, têm grau 1. Observe que uma restrição simples é sempre uma árvore filogenética (ou seja, todos os seus nós distintos da raiz têm grau diferente de 1).

Seja $\mathcal{G}$ uma floresta rotulada, e $\mathcal{S}=\left\{S_{1}, \ldots, S_{m}\right\}$ uma família não-vazia de subconjuntos de $S_{\mathcal{G}}$ tais que:

- $S_{i}$ é um subconjunto não-vazio dos rótulos de algum componente de $\mathcal{G}$ para $i=1, \ldots, m$;

- as projeções simples $\mathcal{G}\left[S_{i}\right]$ são duas a duas disjuntas nos nós.

Então:

- dizemos que $\mathcal{S}$ define uma restrição em $\mathcal{G}$;

- a projeção de $\mathcal{S}$ sobre $\mathcal{G}$, denotada por $\mathcal{G}[\mathcal{S}]$, é a floresta enraizada constituída dos componentes $\mathcal{G}\left[S_{i}\right]$;

- a restrição de $\mathcal{G}$ a $\mathcal{S}$, denotada por $\mathcal{G} \mid \mathcal{S}$, é a floresta rotulada constituída dos componentes $\mathcal{G} \mid S_{i}$.

Dizemos que uma floresta rotulada $\mathcal{G}^{\prime}$ é uma restrição de $\mathcal{G}$ se $\mathcal{G}^{\prime}=\mathcal{G} \mid \mathcal{S}$ para alguma família $\mathcal{S}$ de subconjuntos de $S_{\mathcal{G}}$ que define uma restrição em $\mathcal{G}$. Observe que neste caso temos $\mathcal{S}_{\mathcal{G}^{\prime}}=\mathcal{S}$.

Note que dada uma floresta rotulada $\mathcal{G}$, nem toda família de subconjuntos de $S_{\mathcal{G}}$ define uma restrição em $\mathcal{G}$. Para que um conjunto $S$ defina uma restrição simples em $\mathcal{G}$, é preciso que $S \subseteq S_{\mathcal{W}}$ para algum componente $\mathcal{W}$ de $\mathcal{G}$. Por exemplo, se $\mathcal{G}$ é a floresta mostrada na Figura 3.1, então os conjuntos $\{1,2,5,6\}$ e $\{3,4,7\}$ definem restrições simples de $\mathcal{G}$; o mesmo não ocorre com o conjunto $\{1,2,3,9,10\}$.

No caso de restrições não simples, é necessário que cada conjunto da família defina uma restrição simples e também que as respectivas projeções simples sejam disjuntas nos nós. Por exemplo, a família $\mathcal{S}:=\{\{1,2,5,6\} ;\{3,4,7\}\}$ não define uma restrição na floresta rotulada $\mathcal{G}$ indicada na Figura 3.1. Esta família satisfaz a primeira condição das definições de restrição mas não satisfaz a segunda: $\mathcal{G}\left[S_{1}\right]$ e $\mathcal{G}\left[S_{2}\right]$ intersectam-se nos nós $w, z, v, y$ e $t$.

Seja $\mathcal{G}$ uma floresta rotulada. O tamanho de uma restrição $\mathcal{G}^{\prime}$ de $\mathcal{G}$, denotado por $\left|\mathcal{G}^{\prime}\right|$, é o número de componentes de $\mathcal{G}^{\prime}$. Se $S_{\mathcal{G}^{\prime}}=S_{\mathcal{G}}$, então $\mathcal{G}^{\prime}$ é uma restrição completa de $\mathcal{G}$. Se uma família $\mathcal{S}$ de subconjuntos de $S_{\mathcal{G}}$ define uma restrição $\mathcal{G}^{\prime}$ em $\mathcal{G}$ tal que $S_{\mathcal{G}^{\prime}}=S_{\mathcal{G}}$, então dizemos que $\mathcal{S}$ define uma restrição completa em $\mathcal{G}$.

Uma restrição é trivial se ela é uma floresta filogenética trivial. 

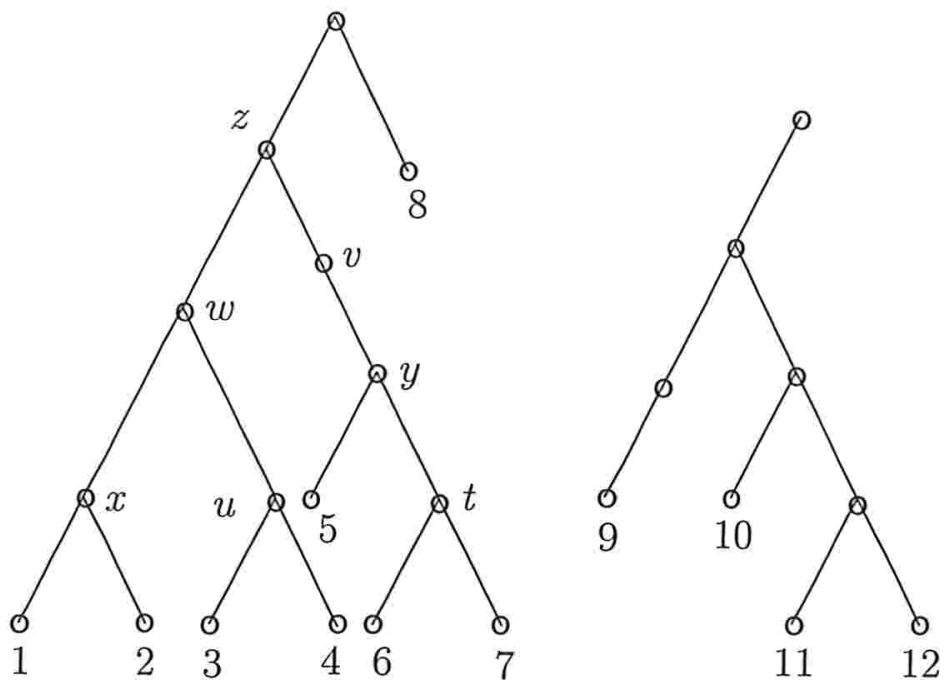

Floresta rotulada $\mathcal{G}$.

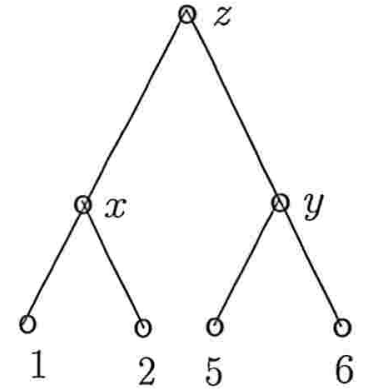

Restrição simples $\mathcal{G} \mid S_{1}$.

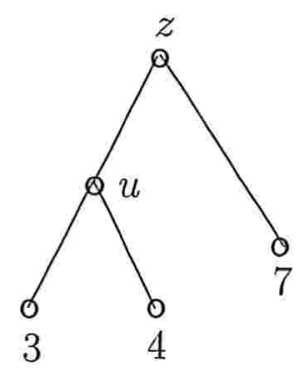

Restrição simples $\mathcal{G} \mid S_{2}$.

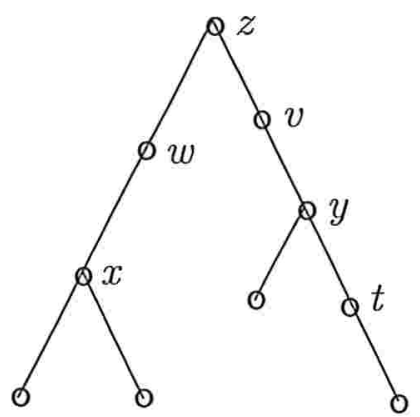

Projeção simples $\mathcal{G}\left[S_{1}\right]$.

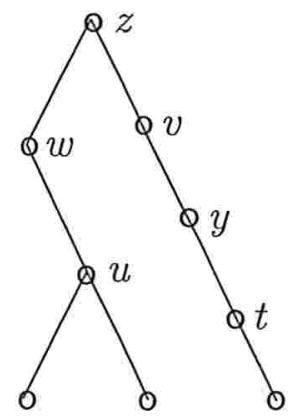

Projeção simples $\mathcal{G}\left[S_{2}\right]$.

Figura 3.1: Exemplos de restrição simples e projeção simples $\left(S_{1}:=\{1,2,5,6\} ; S_{2}:=\right.$ $\{3,4,7\})$. 
Os seguintes lemas podem ser facilmente provados a partir das definições de restrição e projeção.

Lema 3.1 Seja $\mathcal{G}$ uma floresta rotulada. Então:

1) $T_{\mathcal{G}}=\mathcal{G}\left[\mathcal{S}_{\mathcal{G}}\right]$ se e somente se as raízes dos componentes de $\mathcal{G}$ têm grau diferente de 1;

2) $\mathcal{G}=\mathcal{G} \mid \mathcal{S}_{\mathcal{G}}$ se e somente se $\mathcal{G}$ é uma floresta filogenética.

Prova. Vamos provar o lema apenas para o caso em que $\mathcal{G}$ tem um único componente. Neste caso, $S_{\mathcal{G}}$ define uma restrição em $\mathcal{G}$.

Suponhamos que $r_{\mathcal{G}}$ tem grau 1. Então $r_{\mathcal{G}}$ não é o antepassado comum mais recente de um subconjunto de folhas de $\mathcal{G}$, e portanto só pode pertencer a $\mathcal{G}\left[S_{\mathcal{G}}\right]$ se estiver em algum caminho entre dois antepassados comuns mais recentes. Mas todos os nós de $\mathcal{G}$ deste tipo são descendentes estritos de $r_{\mathcal{G}}$, e portanto nenhum caminho entre dois deles passa por $r_{\mathcal{G}}$. Deste modo, $r_{\mathcal{G}}$ não está em $\mathcal{G}\left[S_{\mathcal{G}}\right]$, e portanto $T_{\mathcal{G}} \neq \mathcal{G}\left[S_{\mathcal{G}}\right]$.

Suponhamos agora que $r_{\mathcal{G}}$ tem grau diferente de 1 . Temos que $\mathcal{G}$ preserva os antepassados comuns mais recentes dos subconjuntos de folhas de $\mathcal{G}$ e os caminhos entre eles. Resta provar que todos os nós de $\mathcal{G}$ estão em $\mathcal{G}\left[S_{\mathcal{G}}\right]$; se provarmos isso, então temos $T_{\mathcal{G}}=\mathcal{G}\left[S_{\mathcal{G}}\right]$ pela minimalidade de $\mathcal{G}\left[S_{\mathcal{G}}\right]$. Como todo nó de grau diferente de 1 em $\mathcal{G}$ é um antepassado comum mais recente, enquanto que todo nó de grau 1 em $\mathcal{G}$ pertence a um caminho entre dois destes antepassados (por exemplo, a raiz e uma folha descendente do nó em questão), temos que todos os nós de $\mathcal{G}$ aparecem em $\mathcal{G}\left[S_{\mathcal{G}}\right]$. Isso conclui a prova do item (1).

Para a prova do item (2), suponha que $\mathcal{G}=\mathcal{G} \mid S_{\mathcal{G}}$. Então é imediato que $\mathcal{G}$ é uma floresta filogenética, pois $\mathcal{G} \mid S_{\mathcal{G}}$ não pode ter nós de grau 1 já que preserva somente os antepassados comuns mais recentes dos subconjuntos de folhas de $\mathcal{G}$, não preservando os caminhos entre estes antepassados.

Vamos mostrar a outra implicação. Observe que $\mathcal{G}$ não tem nós de grau 1, pois é uma árvore filogenética. Neste caso, todos os nós de $\mathcal{G}$ são antepassados comuns mais recentes de subconjuntos de folhas, e portanto pertencem a $\mathcal{G} \mid S_{\mathcal{G}}$. Como as relações de adjacência entre os nós deste tipo têm que ser mantidas para que esses antepassados sejam preservados, então $\mathcal{G}=\mathcal{G} \mid S_{\mathcal{G}}$.

A floresta rotulada $\mathcal{G}$ na Figura 3.1 é um exemplo para o qual $T_{\mathcal{G}} \neq \mathcal{G}\left[\mathcal{S}_{\mathcal{G}}\right]$.

Lema 3.2 Seja $\mathcal{G}$ uma floresta rotulada, e um arco de $\mathcal{G}$, e $\mathcal{S}$ uma família de subconjuntos de $S_{\mathcal{G}}$ que define uma restrição em $\mathcal{G}$. Então existe no máximo um conjunto $S \in \mathcal{S}$ tal que $S \cap D_{e} \neq \emptyset$ e $S \backslash D_{e} \neq \emptyset$. 
Prova. Suponhamos que existam $S_{1}$ e $S_{2}$ em $\mathcal{S}$ distintos que satisfaçam o enunciado. Sejam $x_{1} \in S_{1} \cap D_{e}, y_{1} \in S_{1} \backslash D_{e}, x_{2} \in S_{2} \cap D_{e}$ e $y_{2} \in S_{2} \backslash D_{e}$. Considere os caminhos de $x_{1}$ a $y_{1}$ em $\mathcal{G}\left[S_{1}\right]$ e de $x_{2}$ a $y_{2}$ em $\mathcal{G}\left[S_{2}\right]$. Estes dois caminhos devem conter o arco $e$, e portanto eles não são disjuntos. Por outro lado, $\mathcal{G}\left[S_{1}\right]$ e $\mathcal{G}\left[S_{2}\right]$ são disjuntas nos nós, o que é uma contradição.

Seja $\mathcal{G}$ uma floresta rotulada, $S$ um subconjunto de $S_{\mathcal{G}}$ que define uma restrição simples em $\mathcal{G}$, e $\mathcal{G}^{\prime}$ uma restrição completa de $\mathcal{G}$. A restrição de $\mathcal{G} \mid S$ definida pela família de conjuntos $\left\{S^{\prime} \cap S: S^{\prime} \in \mathcal{S}_{\mathcal{G}^{\prime}}\right.$ e $\left.S^{\prime} \cap S \neq \emptyset\right\}$ é a restrição induzida por $\mathcal{G}^{\prime}$ sobre $\mathcal{G} \mid S$. A Figura 3.2 mostra um exemplo de restrição induzida; $\mathcal{G}^{\prime}$ é indicada em negrito.

\subsection{Florestas de concordância ótima}

Sejam $\mathcal{G}$ e $\mathcal{H}$ duas florestas filogenéticas tais que $S_{\mathcal{G}}=S_{\mathcal{H}}$. Uma floresta filogenética de concordância (ou, simplesmente, floresta de concordância) de $\mathcal{G}$ e $\mathcal{H}$ é uma floresta filogenética $\mathcal{F}$ tal que $\mathcal{S}_{\mathcal{F}}$ define restrições completas em $\mathcal{G}$ e em $\mathcal{H}$ e as restrições definidas, $\mathcal{G} \mid \mathcal{S}_{\mathcal{F}}$ e $\mathcal{H} \mid \mathcal{S}_{\mathcal{F}}$, são isomorfas. O tamanho de uma floresta de concordância $\mathcal{F}$, denotado por $|\mathcal{F}|$, é o número de componentes de $\mathcal{F}$. Uma floresta de concordância ótima é uma floresta de concordância de tamanho mínimo.

A Figura 3.3 mostra exemplos de florestas de concordância ótimas e não-ótimas de duas árvores filogenéticas. A família $\{\{1,2,3\} ;\{4\} ;\{5\} ;\{6,7\}\}$ define uma floresta de concordância de tamanho 4 para as duas árvores filogenéticas nesta figura. Esta floresta entretanto não é ótima, pois as árvores admitem uma restrição completa com três componentes, definida pela família $\{\{1,2,3,5\} ;\{4\} ;\{6,7\}\}$.

O problema da floresta de concordância ótima consiste no seguinte:

Dadas duas árvores filogenéticas $\mathcal{T}$ e $\mathcal{U}$ tais que $S_{\mathcal{T}}=S_{\mathcal{U}}$, construir uma floresta de concordância ótima de $\mathcal{T}$ e $\mathcal{U}$.

Chamamos este problema abreviadamente de problema MAF (Maximum Agreement Forest problem).

Neste trabalho vamos estudar o problema da floresta de concordância ótima para árvores filogenéticas com grau no máximo $d(d \geq 2)$, denotado por MAF- $d$ (Maximum Agreement Forest problem for trees with node degrees bounded by $d$ ), e definido a seguir:

Dadas duas árvores filogenéticas $\mathcal{T}$ e $\mathcal{U}$ com $S_{\mathcal{T}}=S_{\mathcal{U}}$, cujos nós têm grau no máximo $d$, construir uma floresta de concordância ótima de $\mathcal{T}$ e $\mathcal{U}$. 


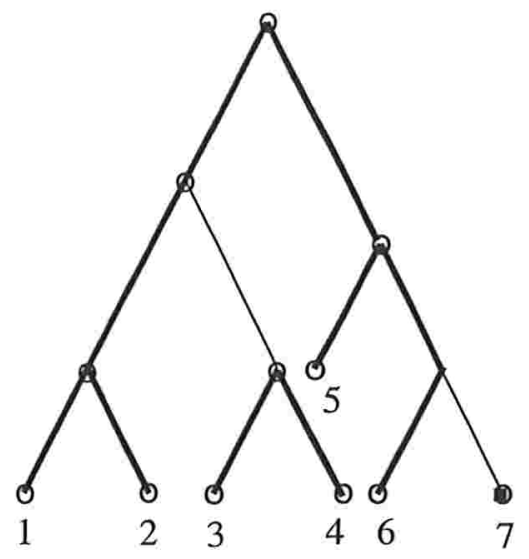

$$
\begin{gathered}
S_{\mathcal{G}}=\{1,2,3,4,5,6,7\} . \\
\mathcal{S}_{\mathcal{G}^{\prime}}=\{\{1,2,5,6\} ;\{3,4\} ;\{7\}\} .
\end{gathered}
$$

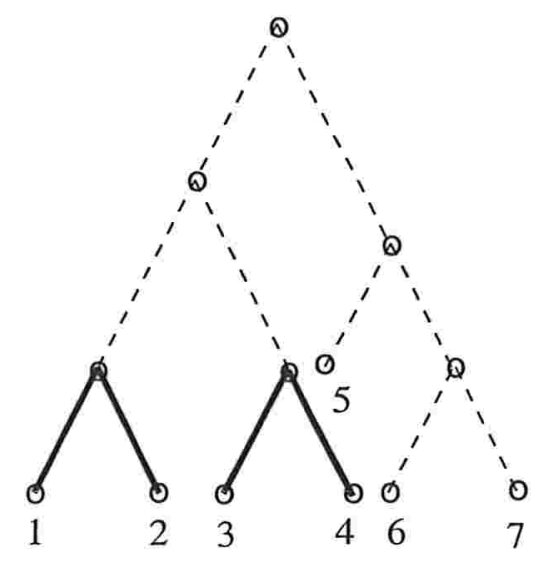

Restrição induzida por $\mathcal{G}^{\prime}$ sobre $\mathcal{G} \mid S$, sendo $S=\{1,2,3,4\}$.

Figura 3.2: Exemplo de restrição induzida. 


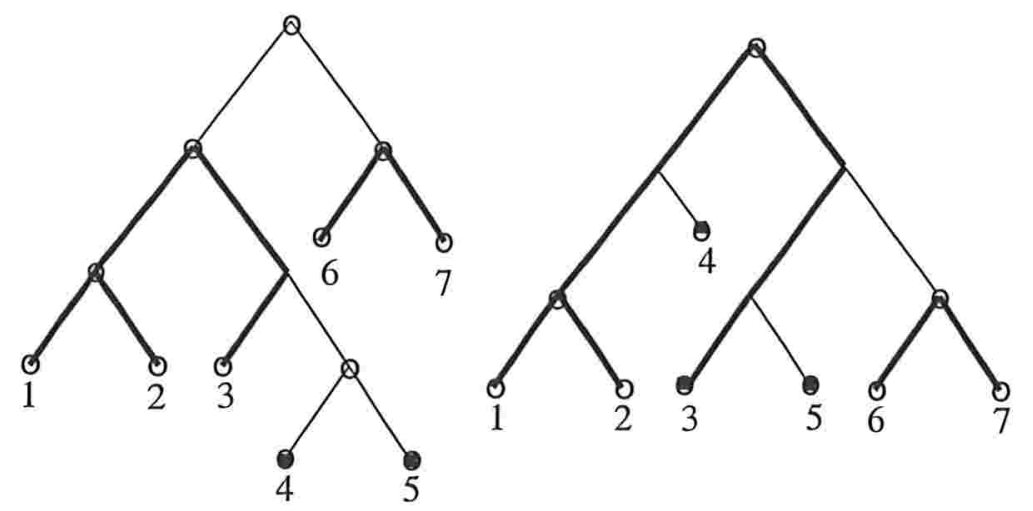

Família $\{\{1,2,3\} ;\{4\} ;\{5\} ;\{6,7\}\}$.

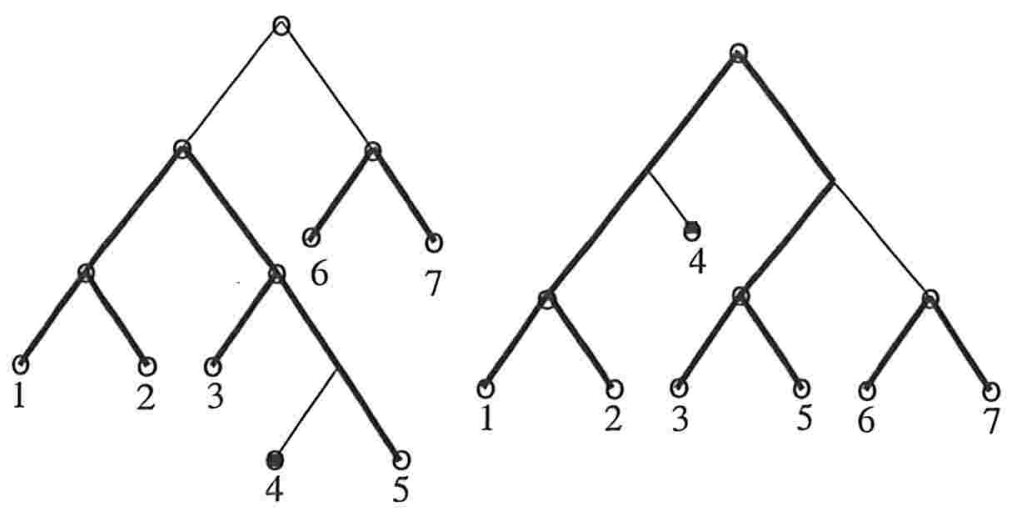

Família $\{\{1,2,3,5\} ;\{4\} ;\{6,7\}\}$.

Figura 3.3: Exemplos de florestas de concordância. 

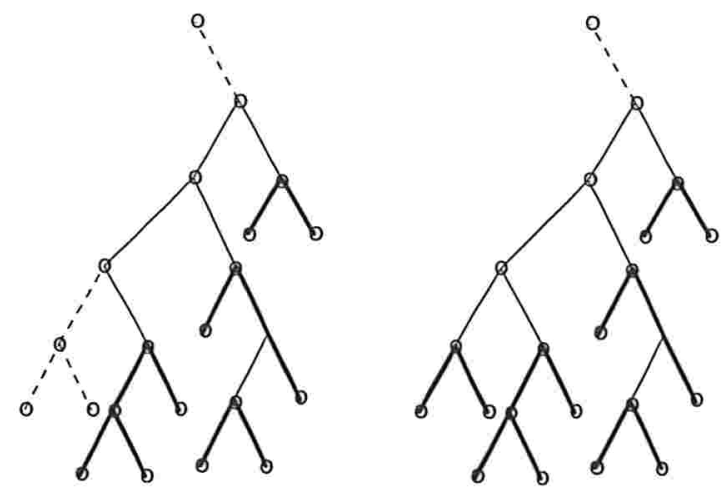

Figura 3.4: Exemplos de arcos de ligação em uma árvore rotulada com duas restrições diferentes.

\subsection{Arcos de ligação}

Nesta seção vamos estudar os objetos que conectam os componentes de uma restrição dentro de uma floresta rotulada: os arcos de ligação.

Se $\mathcal{G}$ é uma floresta rotulada e $\mathcal{S}$ é uma família de subconjuntos de $S_{\mathcal{G}}$ que define uma restrição em $\mathcal{G}$, então um arco $e$ de $\mathcal{G}$ é um arco de ligação de $\mathcal{G}$ com respeito (ou com relação) a $\mathcal{S}$ se $e$ não pertence a $\mathcal{G}[\mathcal{S}]$ e se existem rótulos $a$ e $b$ pertencentes a subconjuntos distintos $S_{a}$ e $S_{b}$ em $\mathcal{S}$, tais que $e$ pertence ao caminho de $f_{\mathcal{G}}(a)$ a $f_{\mathcal{G}}(b)$ em $\mathcal{G}$. Neste caso também dizemos que $e$ é um arco de ligação com respeito à restrição $\mathcal{G} \mid \mathcal{S}$.

Note que a definição de arco de ligação que acabamos de dar depende da restrição que estivermos considerando (ou da família de conjuntos que define esta restrição). Vamos omitir a indicação da restrição sempre que ela estiver clara pelo contexto.

A Figura 3.4 mostra dois exemplos de restrições em uma mesma árvore rotulada. Os arcos mais escuros pertencem à projeção da restrição, e os arcos em linhas contínuas claras são os arcos de ligação.

O lema a seguir caracteriza arcos de ligação em função dos conjuntos da família $\mathcal{S}$.

Lema 3.3 Seja $\mathcal{G}$ uma floresta rotulada, e um arco de $\mathcal{G}$, e $\mathcal{S}$ uma família de subconjuntos de $S_{\mathcal{G}}$ que define uma restrição em $\mathcal{G}$. Então e é um arco de ligação com respeito a $\mathcal{S}$ se e somente se as seguintes condições são satisfeitas:

1) para todo conjunto $S$ em $\mathcal{S}$ temos que $S \cap D_{e}=\emptyset$ ou $S \backslash D_{e}=\emptyset ; e$

2) existem conjuntos distintos $S_{1}$ e $S_{2}$ em $\mathcal{S}$ tais que $S_{1} \cap D_{e} \neq \emptyset$ e $S_{2} \backslash D_{e} \neq \emptyset$.

Prova. Se $e$ é um arco de ligação, então por definição $e$ não pertence a $\mathcal{G}[\mathcal{S}]$. Neste caso o primeiro item da caracterização acima é válido. Para o segundo item, sejam 
$a$ e $b$ dados na definição de arco de ligação. Supondo sem perda de generalidade que $f_{\mathcal{G}}(a) \in D_{e}$, temos que $f_{\mathcal{G}}(b) \notin D_{e}$, pois $e$ pertence ao caminho de $f_{\mathcal{G}}(a)$ a $f_{\mathcal{G}}(b)$ em $\mathcal{G}$. Tomamos $S_{1}:=S_{a}$ e $S_{2}:=S_{b}$, onde $S_{a}$ e $S_{b}$ são os conjuntos dados na definição de arco de ligação.

Vamos provar agora a outra implicação. Suponhamos por absurdo que $e$ pertence a $\mathcal{G}[\mathcal{S}]$. Seja $S$ o conjunto em $\mathcal{S}$ tal que $\mathcal{G}[S]$ contém $e$. Então temos $S \cap D_{e} \neq \emptyset$ e $S \backslash D_{e} \neq \emptyset$ : qualquer folha de $\mathcal{G}[S]$ que seja descendente de $l(e)$ tem rótulo em $S \cap D_{e}$, e qualquer folha de $\mathcal{G}[S]$ que seja descendente de $r_{\mathcal{G} \mid S}$ mas não de $l(e)$ (tal folha existe pois $r_{\mathcal{G} \mid S}$ tem grau diferente de 1 ) tem rótulo em $S \backslash D_{e}$. Isso contradiz o primeiro item da caracterização; portanto, e não pertence a $\mathcal{G}[\mathcal{S}]$.

Resta exibir dois rótulos que façam o papel de $a$ e $b$ na definição de arco de ligação. Podemos tomar como $a$ um rótulo em $S_{1} \cap D_{e}$ e como $b$ um rótulo em $S_{2} \backslash D_{e}$. Claramente, $e$ pertence ao caminho entre $a$ e $b$. Portanto podemos fazer $S_{a}:=S_{1}$ e $S_{b}:=S_{2}$.

O lema seguinte mostra que, se $\mathcal{G}$ é uma floresta filogenética e $\mathcal{G} \mid \mathcal{S}$ é uma restrição completa, então a segunda condição da definição de arco de ligação pode ser ignorada.

Lema 3.4 Seja $\mathcal{G}$ uma floresta filogenética, e um arco de $\mathcal{G}$, e $\mathcal{S}$ uma família de subconjuntos de $S_{\mathcal{G}}$ que define uma restrição completa em $\mathcal{G}$. Então e é um arco de ligação com respeito a $\mathcal{S}$ se e somente se e não pertence a $\mathcal{G}[\mathcal{S}]$.

Prova. É suficiente mostrarmos que se $e$ não pertence a $\mathcal{G}[\mathcal{S}]$ então $e$ é um arco de ligação.

Para isso, considere rótulos $a$ e $b$ pertencentes a um mesmo componente de $\mathcal{G}$, tais que $a \in D_{e}$ e $b \in S_{\mathcal{G}} \backslash D_{e}$. Então claramente $e$ pertence ao caminho que liga $f_{\mathcal{G}}(a)$ a $f_{\mathcal{G}}(b)$ em $\mathcal{G}$. Como $\mathcal{S}$ define uma restrição completa, então $S_{\mathcal{G}}=\bigcup \mathcal{S}$ e portanto existem conjuntos $S_{a} \in \mathcal{S}$ e $S_{b} \in \mathcal{S}$ tais que $a \in S_{a}$ e $b \in S_{b}$. Como $e$ não pertence a $\mathcal{G}[\mathcal{S}]$, então $S_{a} \neq S_{b}$.

A caracterização abaixo é análoga ao Lema 3.3 para restrições completas de florestas filogenéticas.

Lema 3.5 Seja $\mathcal{G}$ uma floresta filogenética, e um arco de $\mathcal{G}$, e $\mathcal{S}$ uma família de subconjuntos de $S_{\mathcal{G}}$ que define uma restrição completa em $\mathcal{G}$. Então e é um arco de ligação com respeito a $\mathcal{S}$ se e somente se para todo conjunto $S \in \mathcal{S}$ temos que $S \cap D_{e}=\emptyset$ ou $S \backslash D_{e}=\emptyset$.

Prova. O resultado segue do Lema 3.4 e do fato de que $\mathcal{S}$ define uma restrição completa em $\mathcal{G}$. 


\section{Capítulo 4}

\section{Algoritmos de aproximação para árvores com grau no máximo 2}

Neste capítulo vamos apresentar resultados de aproximabilidade para o problema da floresta de concordância ótima para árvores filogenéticas com grau no máximo 2. As operações e algoritmos que serão definidos aqui serão mais adiante estendidos para florestas filogenéticas que admitem nós com grau superior a 2. Neste capítulo e no capítulo seguinte, vamos supor que todas as árvores e florestas filogenéticas têm grau no máximo 2.

\subsection{Eliminações, inserções e transferências de subárvore}

A operação de eliminação de arcos em florestas filogenéticas, que introduzimos nesta seção, é a operação básica em função da qual vamos definir todas as operações usadas pelos algoritmos de aproximação que vamos apresentar neste trabalho.

Seja $\mathcal{G}$ uma floresta filogenética, e um arco de $\mathcal{G}$, e $\mathcal{W}$ o componente de $\mathcal{G}$ que contém $e$. Considere os arcos $e_{1}$ e $e_{2}$ indicados na Figura 4.1. Em todos os casos desta figura, o arco $e_{1}$ é tal que $l(e)$ e $l\left(e_{1}\right)$ são nós irmãos, e o arco $e_{2}$, quando existente, é tal que $l\left(e_{2}\right)=u(e)=u\left(e_{1}\right)$.

A eliminação do arco $e$ de $\mathcal{G}$ é a operação que produz como resultado a floresta filogenética obtida fazendo-se a remoção do arco $e$ seguida da contração do $\operatorname{arco} e_{1}$. Esta operação é denotada por $\operatorname{Elim}(\mathcal{G}, l(e))$.

Note que a eliminação de $e$ em $\mathcal{G}$ produz como resultado a floresta filogenética $\mathcal{G}$ com o componente $\mathcal{W}$ trocado pelos componentes $\mathcal{W} \mid D_{e}$ e $\mathcal{W} \mid\left(S_{\mathcal{W}} \backslash D_{e}\right)$. Veja os esquemas na Figura 4.1.

A remoção do arco $e$ em $\mathcal{W}$ produz como resultado a floresta rotulada com com- 
Capítulo 4. Algoritmos de aproximação para árvores com grau no máximo 237

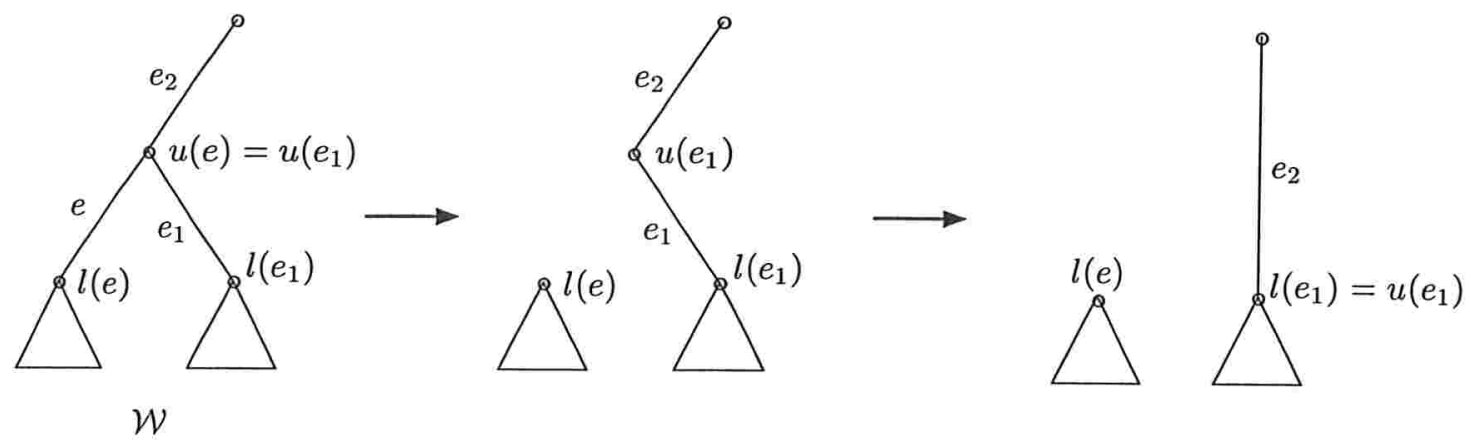

$u(e)$ não é uma raiz.

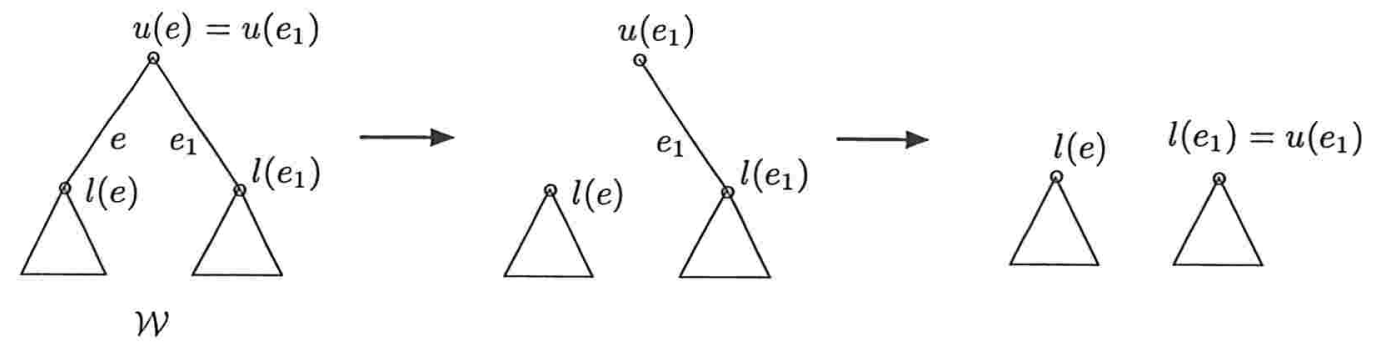

$u(e)$ é uma raiz.

Figura 4.1: Eliminação em árvores filogenéticas com grau no máximo 2. 
ponentes $\mathcal{W}\left[D_{e}\right]$ e $\mathcal{W}\left[S_{\mathcal{W}} \backslash D_{e}\right]$. Observe que estes dois componentes são sempre disjuntos nos nós. Como $u\left(e_{1}\right)$ é o único nó com grau $1 \mathrm{em} \mathcal{W}\left[S_{\mathcal{W}} \backslash D_{e}\right]$, o resultado da contração de $e_{1}$ neste componente é exatamente $\mathcal{W} \mid\left(S_{\mathcal{W}} \backslash D_{e}\right)$, enquanto que $\mathcal{W}\left[D_{e}\right]=\mathcal{W} \mid D_{e}$.

O lema seguinte justifica o uso que faremos, nos algoritmos de aproximação que serão estudados neste trabalho, da operação de eliminação para produzir restrições a partir das árvores filogenéticas dadas como entrada.

Lema 4.1 Se $\mathcal{H}$ é uma floresta flogenética, então:

1) Fazendo uma seqüência de $m$ eliminações a partir de $\mathcal{H}$, temos ao final uma restrição completa de $\mathcal{H}$ de tamanho $|\mathcal{H}|+m$.

2) Seja $\mathcal{S}$ uma familia de conjuntos que define uma restrição completa em $\mathcal{H}$. Então $\mathcal{H} \mid \mathcal{S}$ pode ser obtida a partir de $\mathcal{H}$ através de uma seqüência de $|\mathcal{S}|-|\mathcal{H}|$ eliminações.

Prova. O primeiro item do lema é imediato. A prova do segundo item é feita por indução em $|\mathcal{S}|-|\mathcal{H}|$. Se $|\mathcal{S}|-|\mathcal{H}|=0$, então $\mathcal{S}=\mathcal{S}_{\mathcal{H}}$. Como $\mathcal{H}$ é uma floresta filogenética, então $\mathcal{H}=\mathcal{H} \mid \mathcal{S}$.

Se $|\mathcal{S}|-|\mathcal{H}|>0$, então existe pelo menos um componente $\mathcal{W}$ de $\mathcal{H}$ e pelo menos um componente $\mathcal{W}^{\prime}$ de $\mathcal{H} \mid \mathcal{S}$ tais que $S_{\mathcal{W}^{\prime}} \subseteq S_{\mathcal{W}}$ e $r_{\mathcal{W}^{\prime}} \neq r_{\mathcal{W}}$.

Observe que, neste caso, $r_{\mathcal{W}^{\prime}}=l(e)$ para algum arco $e$ de $\mathcal{W}$. Eliminando o arco $e$, obtemos de $\mathcal{H}$ uma floresta filogenética $\mathcal{H}^{\prime}$ com um componente a mais do que $\mathcal{H}$, e tal que $\mathcal{H}^{\prime}|\mathcal{S}=\mathcal{H}| \mathcal{S}$. Pela hipótese de indução, existe uma seqüência de $|\mathcal{S}|-\left|\mathcal{H}^{\prime}\right|$ eliminações que transforma $\mathcal{H}^{\prime}$ em $\mathcal{H}^{\prime} \mid \mathcal{S}$. Acrescentando a eliminação do arco $e$ no início da seqüência dada pela hipótese de indução, obtemos uma seqüência de $|\mathcal{S}|-|\mathcal{H}|$ eliminações que transforma $\mathcal{H}$ em $\mathcal{H} \mid \mathcal{S}$.

O lema abaixo apresenta uma importante relação entre seqüências de eliminações que produzem restrições completas triviais a partir de uma árvore filogenética e os arcos de ligação definidos por uma restrição completa desta árvore. Observe que uma seqüência de $\left|S_{\mathcal{H}}\right|-|\mathcal{H}|$ eliminações a partir de $\mathcal{H}$ produz uma restrição completa de $\mathcal{H}$ com $\left|S_{\mathcal{H}}\right|$ componentes, e portanto trivial, de acordo com o Lema 4.1. Este lema será usado na análise da razão de aproximação dos algoritmos que vamos apresentar.

Outro conceito importante na análise é o da preservação de arcos de ligação. Seja $\mathcal{H}$ uma floresta filogenética, $\mathcal{S}$ uma família de subconjuntos de $S_{\mathcal{H}}$ que define uma restrição completa em $\mathcal{H}$, e um arco de $\mathcal{H}, \mathcal{H}^{\prime}$ a restrição obtida como resultado da eliminação do $\operatorname{arco} e$ em $\mathcal{H}$, e $\mathcal{S}^{\prime}$ definido a seguir: 
$\mathcal{S}^{\prime}:= \begin{cases}\mathcal{S} & \text { se e é um arco de ligação de } \mathcal{H} \text { com relação a } \mathcal{S} ; \\ (\mathcal{S} \backslash S) \cup\left\{S \cap D_{e}, S \backslash D_{e}\right\} & \text { caso contrário, sendo } S \text { tal que está em } \mathcal{H}[S] .\end{cases}$

Claramente, os arcos de ligação definidos por $\mathcal{S}$ em $\mathcal{H}$ exceto os arcos $e$ e $e_{1}$, sendo $e_{1}$ o arco indicado na Figura 4.1, são arcos de ligação de $\mathcal{H}^{\prime}$ com respeito a $\mathcal{S}^{\prime}$. Dizemos neste caso que $\mathcal{H}^{\prime}$ preserva os arcos de ligação definidos por $\mathcal{S}$ (ou, alternativamente, por $\mathcal{H} \mid \mathcal{S})$ em $\mathcal{H}$.

Vamos agora considerar uma seqüência de $p \geq 1$ eliminações feita a partir de $\mathcal{H}$. Seja $\mathcal{H}^{1}:=\mathcal{H}$, e para cada $j=2, \ldots, p+1$, seja $\mathcal{H}^{j}$ o resultado da $(j-1)$-ésima eliminação sobre $\mathcal{H}^{j-1}$. Seja $\mathcal{S}^{1}:=\mathcal{S}$, e para cada $j=2, \ldots, p+1$, seja $\mathcal{S}^{j}$ definido a seguir:

$\mathcal{S}^{j}:= \begin{cases}\mathcal{S}^{j-1} & \text { se o arco } e \text { eliminado em } \mathcal{H}^{j-1} \text { é um arco de } \\ \left(\mathcal{S}^{j-1} \backslash S\right) \cup\left\{S \cap D_{e}, S \backslash D_{e}\right\} & \text { ligação com relação a } \mathcal{S}^{j-1} ; \\ & \text { caso contrário, sendo } S \text { tal que e está } \\ & \text { em } \mathcal{H}^{j-1}[S] .\end{cases}$

Para cada $j=2, \ldots, p+1$, temos que $\mathcal{H}^{j}$ preserva os arcos de ligação definidos por $\mathcal{S}^{j-1}$ em $\mathcal{H}^{j-1}$. Neste caso, os arcos de ligação definidos por $\mathcal{S}$ em $\mathcal{H}$, exceto os arcos eliminados a cada passo bem como os seus arcos irmãos, são arcos de ligação de $\mathcal{H}^{p+1}$ com respeito a $\mathcal{S}^{p+1}$. Dizemos neste caso que $\mathcal{H}^{p+1}$ preserva os arcos de ligação definidos por $\mathcal{S}$ (ou, alternativamente, por $\mathcal{H} \mid \mathcal{S}$ ) em $\mathcal{H}$.

Lema 4.2 Seja $\mathcal{H}$ uma floresta filogenética, e $\mathcal{S}$ uma familia de subconjuntos de $S_{\mathcal{H}}$ que define uma restrição completa em $\mathcal{H}$. Se uma seqüência de $\left|S_{\mathcal{H}}\right|-|\mathcal{H}|$ eliminações é executada a partir de $\mathcal{H}$, então $|\mathcal{S}|-|\mathcal{H}|$ arcos de ligação com relação a $\mathcal{S}$ são eliminados.

Prova. A prova é feita por indução em $\left|S_{\mathcal{H}}\right|-|\mathcal{H}|$. Se $\left|S_{\mathcal{H}}\right|-|\mathcal{H}|=0$, então nenhuma eliminação é executada sobre $\mathcal{H}$. Além disso $\mathcal{H}$ é trivial, portanto $\mathcal{S}=\mathcal{S}_{\mathcal{H}}$ e $|\mathcal{S}|-|\mathcal{H}|=$ 0 .

Se $\left|S_{\mathcal{H}}\right|-|\mathcal{H}|>0$, então pelo menos uma eliminação é executada a partir de $\mathcal{H}$. Neste caso, seja $\mathcal{H}^{\prime}$ a floresta filogenética obtida na primeira eliminação executada a partir de $\mathcal{H}$. Se o arco e eliminado nesta operação é um arco de ligação com relação a $\mathcal{S}$, então seja $\mathcal{S}^{\prime}:=\mathcal{S}$. Caso contrário, seja $S$ o único elemento de $\mathcal{S}$ tal que $S \cap D_{e} \neq \emptyset$ e $S \backslash D_{e} \neq \emptyset$. O Lema 3.5 garante a existência de $S$, enquanto que o Lema 3.2 garante a sua unicidade. Seja $\mathcal{S}^{\prime}:=\mathcal{S} \backslash S \cup\left\{S \cap D_{e}, S \backslash D_{e}\right\}$. Claramente, $\mathcal{S}^{\prime}$ define uma restrição completa em $\mathcal{H}$ que preserva os arcos de ligação definidos por $\mathcal{S}$. 
É fácil ver que $\mathcal{S}^{\prime}$ define uma restrição completa em $\mathcal{H}^{\prime}$ e que $\left|S_{\mathcal{H}^{\prime}}\right|-\left|\mathcal{H}^{\prime}\right|=$ $\left|S_{\mathcal{H}}\right|-|\mathcal{H}|-1$. Portanto, pela hipótese de indução, $\left|\mathcal{S}^{\prime}\right|-\left|\mathcal{H}^{\prime}\right|$ arcos de ligação são eliminados em $\mathcal{H}^{\prime}$ depois que as eliminações restantes são executadas a partir de $\mathcal{H}^{\prime}$. Se $e$ é um arco de ligação com relação a $\mathcal{S}$, então $\left|\mathcal{S}^{\prime}\right|-\left|\mathcal{H}^{\prime}\right|+1=\left|\mathcal{S}^{\prime}\right|-\left(\left|\mathcal{H}^{\prime}\right|-1\right)=|\mathcal{S}|-|\mathcal{H}|$ arcos de ligação são eliminados em $\mathcal{H}$, caso contrário $\left|\mathcal{S}^{\prime}\right|-\left|\mathcal{H}^{\prime}\right|=(|\mathcal{S}|+1)-(|\mathcal{H}|+1)=$ $|\mathcal{S}|-|\mathcal{H}|$ arcos de ligação são eliminados em $\mathcal{H}$.

A seguir vamos apresentar a operação de transferência de subárvore. Esta operação é de grande importância no contexto do modelo de Hein para a evolução de seqüências de DNA sujeitas a recombinação. Um estudo desta operação e de sua relação com florestas de concordância será apresentado nos Capítulos 8 e 9. Preferimos definir esta operação na presente seção, pois ela é empregada no gerador de instâncias do problema MAF-2 que implementamos, o qual descrevemos no Capítulo 5.

Para definir transferências de subárvore, precisamos definir uma operação adicional, a operação de inserção de componentes em florestas filogenéticas. Seja $\mathcal{G}$ uma floresta filogenética, $v$ um nó de $\mathcal{G}, \mathcal{V}$ o componente de $\mathcal{G}$ que contém $v$, e $\mathcal{Y}$ uma árvore filogenética disjunta de $\mathcal{G}$ nos nós. A inserção do componente $\mathcal{Y}$ junto ao nó $v$ em $\mathcal{G}$ produz como resultado a floresta filogenética obtida com a seguinte seqüência de operações:

- Extensão do nó $v$ em $\mathcal{G}$. Vamos denotar por $\mathcal{G}^{*}$ o resultado desta operação, por $x$ e $y$ os dois novos nós que substituem o nó $v$ em $\mathcal{G}^{*}$, sendo $y$ o antecessor imediato de $x$ em $\mathcal{G}^{*}$, e por $\mathcal{V}^{*}$ o componente de $\mathcal{G}^{*}$ que contém os nós $x$ e $y$. Temos que se $v$ é raiz de $\mathcal{V}$ então $y$ é raiz de $\mathcal{V}^{*}$.

- Conexão de $\mathcal{Y}$ em $\mathcal{G}^{*}$ através do nó $y$.

A Figura 4.2 esquematiza esta operação para os casos em que $v \neq r_{\mathcal{V}}$ e em que $v=r_{\mathcal{V}}$.

Observe que a inserção é a operação inversa da eliminação. De fato, se $\mathcal{G}^{\prime}$ é o resultado de $\operatorname{Elim}(\mathcal{G}, l(e))$ e $\mathcal{W}^{\prime}$ é o componente de $\mathcal{G}^{\prime}$ que contém $l(e)$, então o resultado da inserção de $\mathcal{W}^{\prime}$ em $\mathcal{G}^{\prime}$ junto ao nó $l\left(e_{1}\right)$ é $\mathcal{G}$, sendo $e$ o arco indicado na Figura 4.1.

Seja $\mathcal{W}$ uma árvore filogenética, e $x$ e $y$ dois nós de $\mathcal{W}$ (não necessariamente distintos) tais que $x \neq r_{\mathcal{W}}$ e $y$ não é o antepassado imediato de $x$ em $\mathcal{W}$. A operação de transferência de subárvore em $\mathcal{W}$ definida pelo par de nós $(x, y)$ produz o resultado da seguinte seqüência de operações:

- Eliminação do arco $s_{\mathcal{G}}(x)$, produzindo uma floresta filogenética $\mathcal{W}^{*}$ composta de dois componentes distintos que vamos denominar de $\mathcal{W}_{y}$ e $\mathcal{W}^{\prime}$, sendo $\mathcal{W}_{y}$ o componente de $\mathcal{W}^{*}$ que contém o nó $y$. 
Capítulo 4. Algoritmos de aproximação para árvores com grau no máximo 241

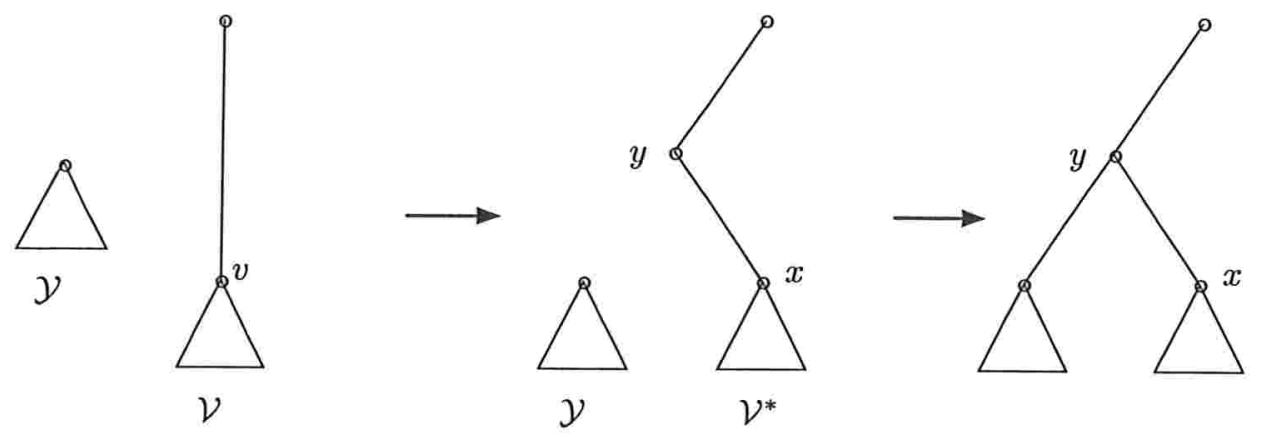

$$
v \neq r_{\mathcal{V}}
$$
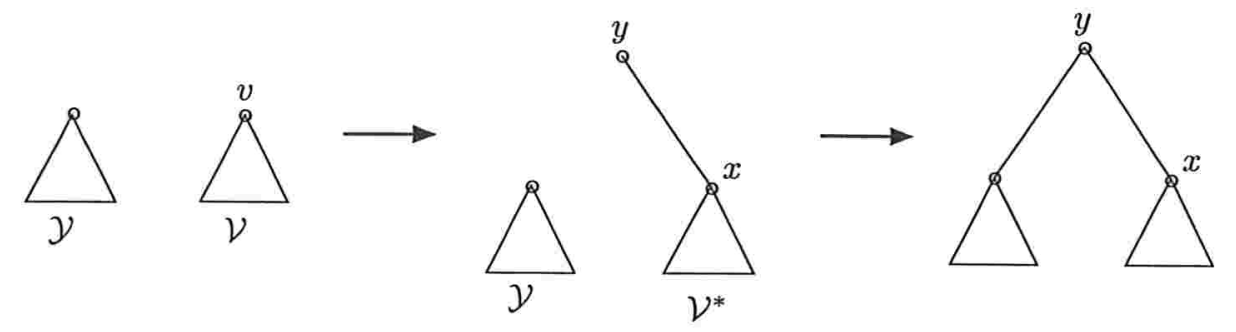

$$
v=r_{\mathcal{V}}
$$

Figura 4.2: Inserção em árvores filogenéticas com grau no máximo 2. 

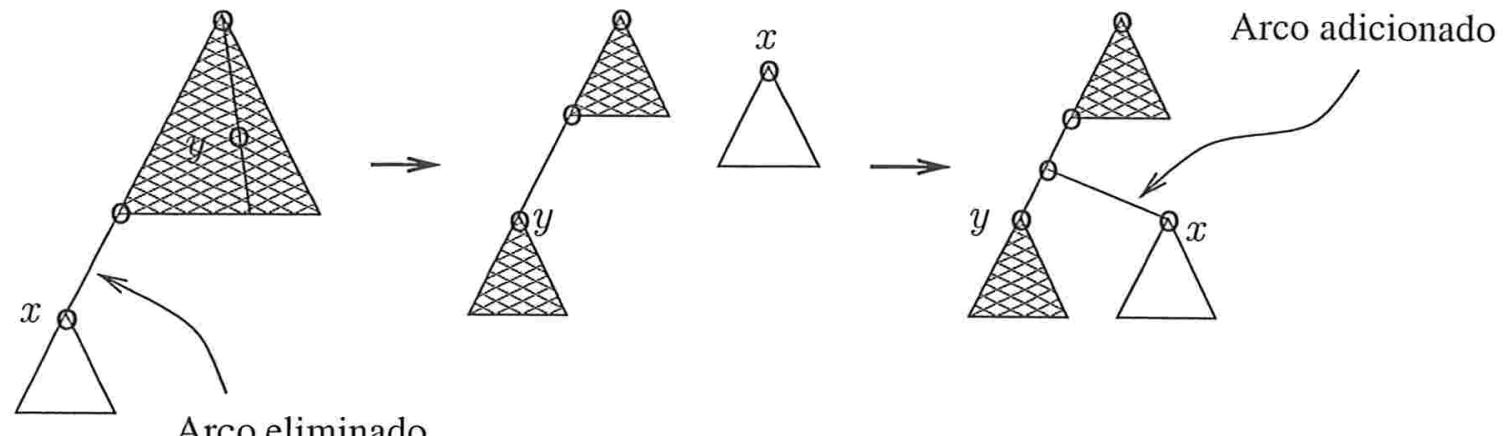

Arco eliminado

$y$ não é um descendente de $x$
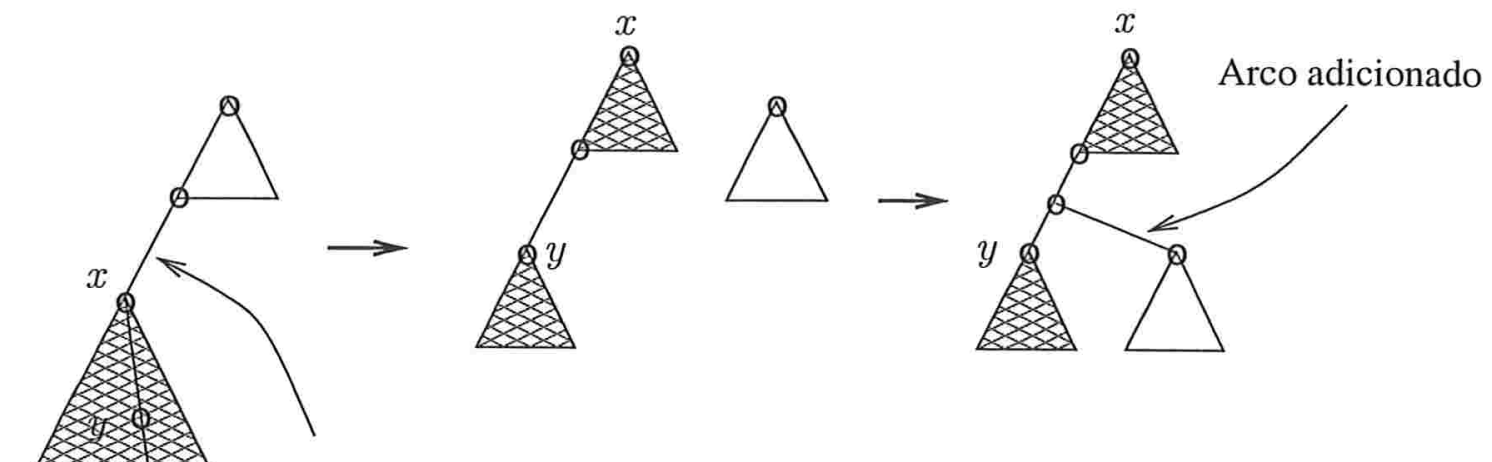

Arco eliminado

$y$ é um descendente de $x$

Figura 4.3: Transferência de subárvore em árvores filogenéticas com grau no máximo 2.

- Inserção do componente $\mathcal{W}^{\prime}$ em $\mathcal{W}_{y}$ junto ao nó $y$.

Denotamos esta operação por $\operatorname{TS}(\mathcal{W}, x, y)$.

A Figura 4.3 mostra dois esquemas para esta operação. Em ambos os esquemas, o componente $\mathcal{W}_{y}$, que contém o nó y após a eliminação, está sombreado. No primeiro esquema, $y$ não é um descendente de $x$, e no segundo, $y$ é um descendente de $x$.

A operação de transferência de subárvore admite uma operação inversa, que é uma outra operação de transferência de subárvore. De fato, se $x^{\prime}$ é o nó irmão de $x$ na árvore filogenética $\mathcal{W}$ e $\mathcal{V}$ é a árvore resultante da operação $\operatorname{TS}(\mathcal{W}, x, y)$, então a operação $\operatorname{TS}\left(\mathcal{V}, x, x^{\prime}\right)$ produz $\mathcal{W}$ como resultado.

Observe que a definição de transferência de subárvore que demos na Introdução deste trabalho é mais restrita do que esta que apresentamos aqui. 


\subsection{O método básico}

Nesta seção vamos apresentar uma família de algoritmos de aproximação para o problema MAF-2. Cada algoritmo desta família aceita como entrada duas árvores filogenéticas $\mathcal{T}$ e $\mathcal{U}$ com $S_{\mathcal{T}}=S_{\mathcal{U}}$, e fornece como saída uma floresta de concordância de $\mathcal{T}$ e $\mathcal{U}$ de tamanho no máximo um múltiplo fixo do tamanho de uma floresta de concordância ótima de $\mathcal{T}$ e $\mathcal{U}$. Nesta família de algoritmos estão inclusos o algoritmo de Hein et al. $[19,20]$ e também o primeiro dos três algoritmos de 3-aproximação que vamos apresentar para o problema MAF-2.

A família de algoritmos de aproximação que vamos mostrar baseia-se no método geral de solução que vamos apresentar em seguida, o qual vamos chamar de método básico. Este método será utilizado em todos os algoritmos que vamos apresentar neste trabalho para construção de florestas de concordância.

O método básico recebe como entrada duas árvores filogenéticas $\mathcal{T}$ e $\mathcal{U}$ com $S_{\mathcal{T}}=S_{\mathcal{U}}$ e devolve como saída o tamanho de uma floresta de concordância de $\mathcal{T}$ e $\mathcal{U}$. Cada iteração do método inicia-se com duas restrições completas obtidas a partir de $\mathcal{T}$ e $\mathcal{U}$ pelas operações (definidas em termos de eliminações, a seguir) executadas durante as iterações anteriores. No início de cada iteração, o método procura um par de rótulos cujas respectivas folhas são irmãs na restrição completa obtida a partir de $\mathcal{T}$. Se o método não encontra um tal par, então ele devolve o tamanho de uma floresta de concordância de $\mathcal{T}$ e $\mathcal{U}$, obtido a partir da contagem de operações de um determinado tipo, e pára. Caso contrário, ele identifica o caso satisfeito na restrição obtida de $\mathcal{U}$ pelo par de rótulos identificado. De acordo com este caso, ele então seleciona e aplica sobre as restrições uma seqüência pré-definida de operações e atualiza a contagem das operações. Cada uma destas seqüências de operações é chamada de transação.

Enquanto que os casos fazem parte do método básico, sendo portanto sempre os mesmos para qualquer algoritmo baseado neste método, as transações são diferentes para cada algoritmo, e em cada algoritmo pode haver uma transação diferente para cada caso. Casos e transações serão discutidos mais adiante.

Definimos a seguir as operações que são realizadas no método básico.

Seja $\mathcal{W}$ uma floresta filogenética, e $e:=x y$ um arco de $\mathcal{W}$. A operação de corte do $\operatorname{arco} e$, denotada por $\operatorname{Cte}(\mathcal{W}, x)$, é a eliminação de $e$. Sejam $x$ e $y$ duas folhas irmãs em $\mathcal{W}$. A operação de encolhimento das folhas $x$ e $y$ para $x$, escrita $\operatorname{Enc}(\mathcal{W}, x, y)$, é a eliminação do arco cuja extremidade inferior é $y$. Observe que há dois modos diferentes de encolher um par $x, y$ de folhas irmãs: podemos encolher o par para o nó $x$ ou para o nó $y$.

Vamos em seguida introduzir a notação para as operações realizadas e as restrições completas obtidas ao longo da execução do método.

As iterações do método básico são numeradas 1, 2, . . e assim por diante. Em 
cada iteração $i \geq 1$, rotulamos as operações feitas, dividindo-as em duas seqüências, $\left(g_{i}^{1}, \ldots, g_{i}^{\bar{\jmath}_{i}}\right)$ e $\left(h_{i}^{1}, \ldots, h_{i}^{\bar{\jmath}_{i}}\right)$, onde $\bar{\jmath}_{i}$ é o máximo entre o número de operações feitas sobre as restrições obtidas de $\mathcal{T}$ e de $\mathcal{U}$ na iteração $i$, e $g_{i}^{j}$ e $h_{i}^{j}$ são respectivamente a $j$-ésima operação aplicada sobre a restrição completa obtida de $\mathcal{T}$ e sobre a restrição completa obtida de $\mathcal{U}$ na iteração $i\left(1 \leq j \leq \bar{j}_{i}\right)$. Conforme veremos adiante, em algumas iterações o comprimento das duas seqüências de operações pode diferir. Neste caso, consideramos que a seqüência com menos operações é preenchida no final com "operações identidade", de modo que as duas seqüências tenham comprimento $\bar{\jmath}_{i}$.

Seja $\mathcal{G}_{1}$ a árvore $\mathcal{T}$, e para $i \geq 2$ seja $\mathcal{G}_{i}$ a restrição completa obtida de $\mathcal{G}_{i-1}$ após a execução sucessiva das operações $g_{i}^{1}, \ldots, g_{i}^{\bar{j}_{i}}$ na iteração $i$. Seja $\mathcal{G}_{i}^{1}$ a floresta filogenética $\mathcal{G}_{i}$, e para $j=2, \ldots, \bar{\jmath}_{i}+1$ seja $\mathcal{G}_{i}^{j}$ a restrição completa obtida de $\mathcal{G}_{i}^{j-1}$ pela aplicação da operação $g_{i}^{j-1}$. Observe que, pela definição, temos que $\mathcal{G}_{i+1}^{1}=\mathcal{G}_{i}^{\frac{i}{j_{i}}+1}$ para todo $i \geq 1$. De modo análogo, definimos as florestas filogenéticas $\mathcal{H}_{i}$ e $\mathcal{H}_{i}^{j}$ para $i \geq 1$ e $j=1, \ldots, \bar{j}_{i}+1$.

Vamos definir os casos, ilustrados na Figura 4.4. Se no início de uma iteração $i$ do método básico não ocorre o término das iterações, então $\mathcal{G}_{i}$ admite ao menos um par de folhas irmãs. Sejam $a$ e $b$ os rótulos destas folhas.

Se $f_{\mathcal{H}_{i}}(a)$ e $f_{\mathcal{H}_{i}}(b)$ pertencem a um mesmo componente de $\mathcal{H}_{i}$, então chamamos de eixo $(a, b)$ o caminho entre $f_{\mathcal{H}_{i}}(a)$ e $f_{\mathcal{H}_{i}}(b)$ em $\mathcal{H}_{i}$, e de hastes do eixo $(a, b)$ os $\operatorname{arcos} e$ de $\mathcal{H}_{i}$ fora do eixo e tais que $u(e)$ pertence ao eixo. As hastes são rotuladas de $s_{1}$ a $s_{k}, k \geq 1$, à medida que suas extremidades superiores se sucedem ao longo do eixo, no sentido de $a$ para $b$.

A Figura 4.4 mostra os cinco casos possíveis no início de cada iteração. O par $a, b$ de rótulos com $f_{\mathcal{G}_{i}}(a)$ e $f_{\mathcal{G}_{i}}(b)$ irmãs em $\mathcal{G}_{i}$ pode ser escolhido arbitrariamente. Os Casos 3 e 4 equivalem à situação em que $f_{\mathcal{H}_{i}}(a)$ e $f_{\mathcal{H}_{i}}(b)$ estão em componentes de $\mathcal{H}_{i}$ distintos. Nos Casos 1, 5 e 2, $f_{\mathcal{H}_{i}}(a)$ e $f_{\mathcal{H}_{i}}(b)$ estão juntas num mesmo componente em $\mathcal{H}_{i}$ e portanto existe o eixo $(a, b)$. No Caso 5 este eixo não possui hastes, e portanto $f_{\mathcal{H}_{i}}(a)$ e $f_{\mathcal{H}_{i}}(b)$ são irmãs. Nos Casos 1 e 2 o eixo possui pelo menos uma haste. $\mathrm{O}$ Caso 1 se distingue do Caso 2 por um limite $\bar{k} \geq 1$ no número de hastes, sendo $\bar{k}$ distinto e fixo para cada algoritmo da família. Os conjuntos de nós indicados por círculos na Figura 4.4 podem ser vazios, enquanto que os conjuntos indicados por triângulos são não-vazios.

A Figura 4.5 descreve o método básico. A existência de uma floresta de concordância de $\mathcal{T}$ e $\mathcal{U}$ cujo tamanho seja o valor devolvido pelo método, bem como a construção de uma tal floresta, dependem da especificação das transações para cada algoritmo e serão abordadas a seguir.

Como já observamos, a definição dos Casos 1 e 2 depende do valor de $\bar{k}$. Na família de algoritmos de aproximação que vamos apresentar, em que os algoritmos são parametrizados por $\bar{k}$, temos transações distintas para esses dois casos. Mais tarde, 


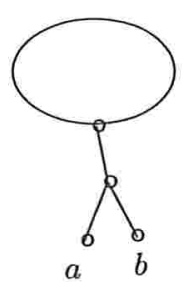

$\mathcal{G}_{i}$

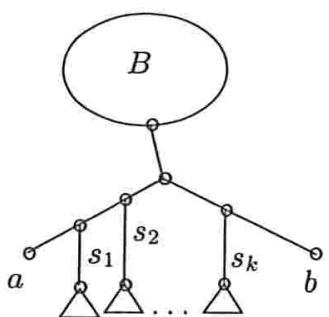

$\mathcal{H}_{i}$
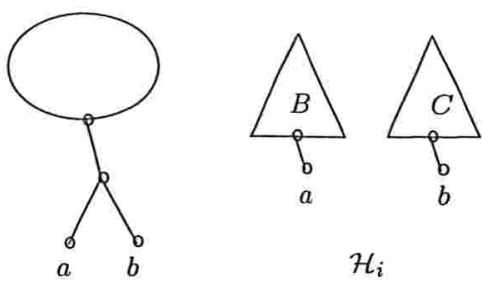

$\mathcal{H}_{i}$

$\mathcal{G}_{i}$

Caso $1(1 \leq k \leq \bar{k})$ e Caso $2(k>\bar{k})$. O Caso 3. Os conjuntos $B$ e $C$ são nãoconjunto $B$ pode ser vazio nos dois casos. vazios.
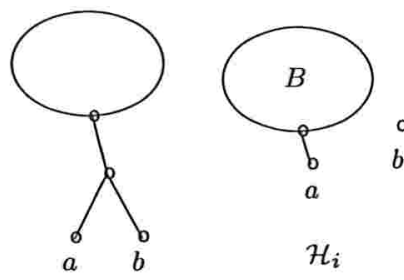

$\mathcal{G}_{i}$

Caso 4. O conjunto $B$ pode ser vazio.
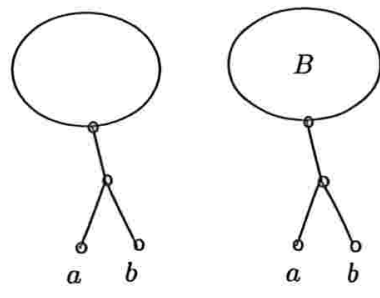

$\mathcal{G}_{i}$
Caso $5(k=0)$. O conjunto $B$ pode ser vazio.

Figura 4.4: Casos do método básico.

Método básico

Entrada: Árvores filogenéticas $\mathcal{T}$ e $\mathcal{U}$ tais que $S_{\mathcal{T}}=S_{\mathcal{U}}$;

Saída: Tamanho de uma floresta de concordância de $\mathcal{T}$ e $\mathcal{U}$.

(1) $\mathcal{G}_{1}:=\mathcal{T}$;

(2) $\mathcal{H}_{1}:=\mathcal{U}$;

(3) $i:=1$;

(4) Enquanto existe um par de folhas irmãs $f_{\mathcal{G}_{i}}(a), f_{\mathcal{G}_{i}}(b)$ em $\mathcal{G}_{i}$

(5) Verifique qual caso é satisfeito por $f_{\mathcal{H}_{i}}(a)$ e $f_{\mathcal{H}_{i}}(b)$ em $\mathcal{H}_{i}$;

(6) Aplique a transação correspondente, obtendo $\mathcal{G}_{i+1}$ a partir de $\mathcal{G}_{i}$ e $\mathcal{H}_{i+1}$ a partir de $\mathcal{H}_{i}$;

(7) $\quad i:=i+1$;

(8) Seja $t$ o número de operações de corte executadas a partir de $\mathcal{U}$;

(9) Devolva $t+1$.

Figura 4.5: O método básico. 


\begin{tabular}{|ll|}
\hline \multicolumn{2}{|l|}{ Transações para o Algoritmo $\mathcal{A}_{\bar{k}}$} \\
\hline Caso 1 & $\operatorname{Cte}\left(\mathcal{H}_{i}^{h}, l\left(s_{h}\right)\right) \operatorname{para} h=1, \ldots, k$ \\
Caso 2 & $\operatorname{Cte}\left(\mathcal{G}_{i}^{1}, f(a)\right) ; \operatorname{Cte}\left(\mathcal{G}_{i}^{2}, f(b)\right) ; \operatorname{Cte}\left(\mathcal{H}_{i}^{1}, f(a)\right) ; \operatorname{Cte}\left(\mathcal{H}_{i}^{2}, f(b)\right)$ \\
Caso 3 & $\operatorname{Cte}\left(\mathcal{G}_{i}^{1}, f(a)\right) ; \operatorname{Cte}\left(\mathcal{G}_{i}^{2}, f(b)\right) ; \operatorname{Cte}\left(\mathcal{H}_{i}^{1}, f(a)\right) ; \operatorname{Cte}\left(\mathcal{H}_{i}^{2}, f(b)\right)$ \\
Caso 4 & $\operatorname{Cte}\left(\mathcal{G}_{i}^{1}, f(b)\right)$ \\
Caso 5 & $\operatorname{Enc}\left(\mathcal{G}_{i}^{1}, f(a), f(b)\right) ; \operatorname{Enc}\left(\mathcal{H}_{i}^{1}, f(a), f(b)\right)$ \\
\hline
\end{tabular}

Tabela 4.1: Transações para o Algoritmo $\mathcal{A}_{\bar{k}}$.

na Seção 4.5, vamos descrever um outro algoritmo que define uma única transação para os Casos 1 e 2.

\subsection{O algoritmo de aproximação $\mathcal{A}_{\bar{k}}$}

Para cada inteiro $\bar{k}, \bar{k} \geq 1$, chamamos de $\mathcal{A}_{\bar{k}}$ o algoritmo que consiste na aplicação do método básico, considerando os casos descritos na Figura 4.4, realizando as transações descritas na Tabela 4.1. Com isso, definimos uma família de algoritmos de construção de florestas de concordância.

O Algoritmo $\mathcal{A}_{1}$ é o algoritmo de Hein et al. [19, 20] para o problema MAF-2. Mais adiante vamos provar que este algoritmo tem razão de aproximação 4, enquanto que o Algoritmo $\mathcal{A}_{2}$ tem razão de aproximação 3. Uma versão preliminar desses dois resultados encontra-se em Rodrigues et al. [31].

\subsubsection{Transações}

Na Tabela 4.1, descrevemos as transações possíveis para cada um dos casos apresentados na Figura 4.4. Os Casos 3, 4 e 5 são tratados da mesma forma por todos os algoritmos desta família. Já os Casos 1 e 2 são tratados de maneira diferente para cada algoritmo, de acordo com o valor de $\bar{k}$. Observe que a operação de encolhimento é utilizada em um contexto bastante particular, apenas no Caso 5, quando detectamos que um par de rótulos define folhas irmãs em ambas as árvores. 


\subsubsection{Prova da correção do algoritmo}

Provamos nesta seção que o Algoritmo $\mathcal{A}_{\bar{k}}$ está correto, e em particular mostramos a existência de uma floresta de concordância cujo tamanho é o valor devolvido pelo método básico. Para isto, considere a seqüência de famílias de subconjuntos de $S_{\mathcal{U}}$ que definimos a seguir.

Seja $\mathcal{S}_{1}:=\mathcal{S}_{1}^{1}:=\left\{\{a\}: a \in S_{\mathcal{U}}\right\}$. Para todo $i$ tal que $i \geq 1$ e $j=2, \ldots, \bar{\jmath}_{i}+1$ seja $\mathcal{S}_{i}^{j}$ a família obtida de $\mathcal{S}_{i}^{j-1}$ da seguinte maneira. Se a operação $h_{i}^{j-1}$ é um corte, então $\mathcal{S}_{i}^{j}:=\mathcal{S}_{i}^{j-1}$. Caso contrário, sejam $x$ e $y$ as duas folhas de $\mathcal{H}_{i}^{j-1}$ tais que $h_{i}^{j-1}=$ $\operatorname{Enc}\left(\mathcal{H}_{i}^{j-1}, x, y\right)$, e sejam $S_{x}$ e $S_{y}$ os conjuntos em $\mathcal{S}_{i}^{j-1}$ que contêm respectivamente $x$ e y. Então $\mathcal{S}_{i}^{j}:=\left(\mathcal{S}_{i}^{j-1} \backslash\left\{S_{x}, S_{y}\right\}\right) \cup\left\{S_{x} \cup S_{y}\right\}$. Para todo $i \geq 2$ seja $\mathcal{S}_{i}^{1}:=\mathcal{S}_{i-1}^{\bar{j}_{i}+1}$.

Nas Figuras 4.6, 4.7 e 4.8 mostramos uma execução do Algoritmo $\mathcal{A}_{2}$ aplicada às árvores da Figura 3.3. Os nós e arcos das restrições $\mathcal{G}_{i}$ e $\mathcal{H}_{i}$ aparecem em negrito, e os componentes de $\mathcal{T}\left[\mathcal{S}_{i}\right]$ e $\mathcal{U}\left[\mathcal{S}_{i}\right]$ estão indicados em cada árvore por linhas curvas.

Na primeira iteração $(i=1), \mathcal{T}\left[\mathcal{S}_{\mathcal{G}_{i}}\right]$ e $\mathcal{U}\left[\mathcal{S}_{\mathcal{H}_{i}}\right]$ são iguais respectivamente às topologias de $\mathcal{T}$ e $\mathcal{U}$, enquanto que $\mathcal{T}\left[\mathcal{S}_{i}\right]$ e $\mathcal{U}\left[\mathcal{S}_{i}\right]$ são restrições completas triviais de $\mathcal{T}$ e $\mathcal{U}$. À medida que a execução do Algoritmo $\mathcal{A}_{\bar{k}}$ prossegue, arcos de $\mathcal{T}\left[\mathcal{S}_{\mathcal{G}_{i}}\right]$ e $\mathcal{U}\left[\mathcal{S}_{\mathcal{H}_{i}}\right]$ são eliminados e componentes de $\mathcal{T}\left[\mathcal{S}_{i}\right]$ e $\mathcal{U}\left[\mathcal{S}_{i}\right]$ são unidos, formando os componentes de uma floresta de concordância de $\mathcal{T}$ e $\mathcal{U}$ cujo tamanho diminui à medida que as iterações se sucedem. Ao término, $\mathcal{T}\left[\mathcal{S}_{i}\right]$ e $\mathcal{U}\left[\mathcal{S}_{i}\right]$ constituem uma floresta de concordância de $\mathcal{T}$ e $\mathcal{U}$, e os componentes de $\mathcal{T}\left[\mathcal{S}_{\mathcal{G}_{i}}\right]$ e $\mathcal{U}\left[\mathcal{S}_{\mathcal{H}_{i}}\right]$ são triviais.

O seguinte lema mostra que a floresta de concordância definida por $\mathcal{S}_{i}$ ao término da execução do Algoritmo $\mathcal{A}_{\vec{k}}$ tem tamanho igual ao valor devolvido pelo algoritmo.

Lema 4.3 Em cada iteração $i \geq 1$ do Algoritmo $\mathcal{A}_{\bar{k}}$, temos que:

1) $S_{\mathcal{G}_{i}}=S_{\mathcal{H}_{i}}$;

2) para cada componente $\mathcal{W}$ de $\mathcal{H}_{i}$ existe um componente $\mathcal{V}$ de $\mathcal{G}_{i}$ tal que $S_{\mathcal{W}} \subseteq S_{\mathcal{V}}$

3) se $j \geq 1$ e $j \leq \bar{\jmath}_{i}$, então $\mathcal{U} \mid \mathcal{S}_{i}$ é uma floresta de concordância de $\mathcal{T}$ e $\mathcal{U}$ de tamanho igual a $\left|S_{\mathcal{U}}\right|$ menos o número de operações de encolhimento executadas a partir de $\mathcal{U}$.

Prova. Os invariantes (1), (2) e (3) podem ser verificados por indução nas seqüências de operações executadas pelo algoritmo a partir das árvores $\mathcal{G}_{1}$ (ou seja, $\mathcal{T}$ ) e $\mathcal{H}_{1}$ (ou seja, $\mathcal{U}$ ). Para $i=1$, temos que $S_{\mathcal{G}_{1}}=S_{\mathcal{T}}=S_{\mathcal{U}}=S_{\mathcal{H}_{1}}$ (invariante (1)); as topologias de $\mathcal{G}_{1}$ e $\mathcal{H}_{1}$ são conexas, portanto os únicos componentes $\mathcal{V}$ de $\mathcal{G}_{1}$ e $\mathcal{W}$ de $\mathcal{H}_{1}$ satisfazem $S_{\mathcal{W}} \subseteq S_{\mathcal{V}}$ (invariante $(2)$ ); e $\mathcal{U} \mid \mathcal{S}_{i}$ é trivial, portanto é uma restrição completa de $\mathcal{U}$ isomorfa a uma restrição completa de $\mathcal{T}$ (invariante (3)). Para $i>1$ 
Capítulo 4. Algoritmos de aproximação para árvores com grau no máximo 248

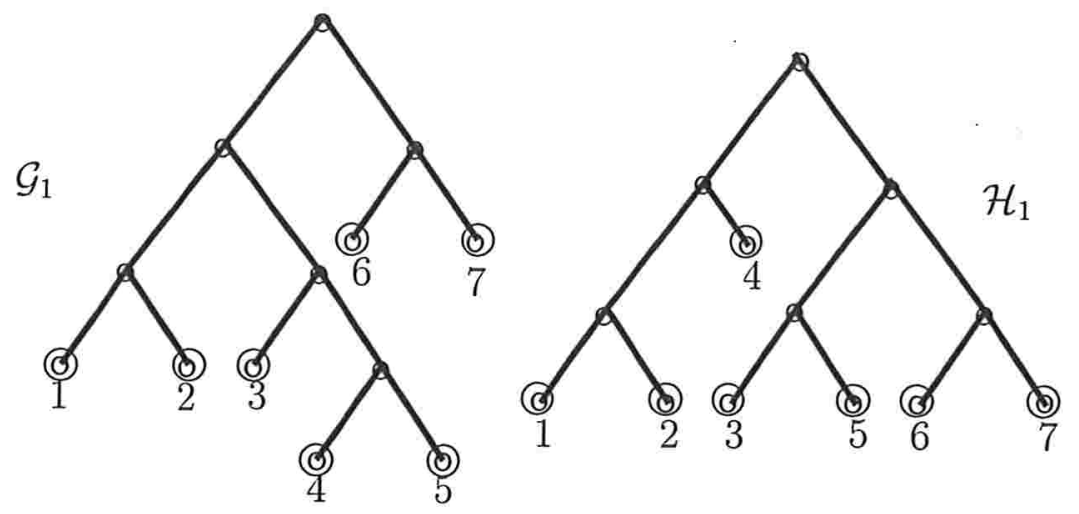

Iteração 1: Par $(1,2)$, caso 5.
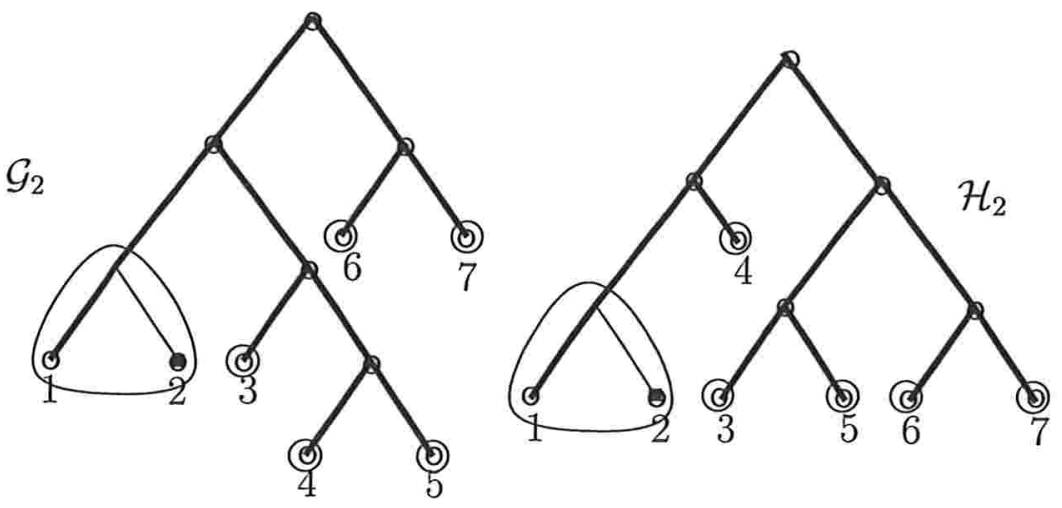

Iteração 2: Par $(4,5)$, caso $2, k=3$.
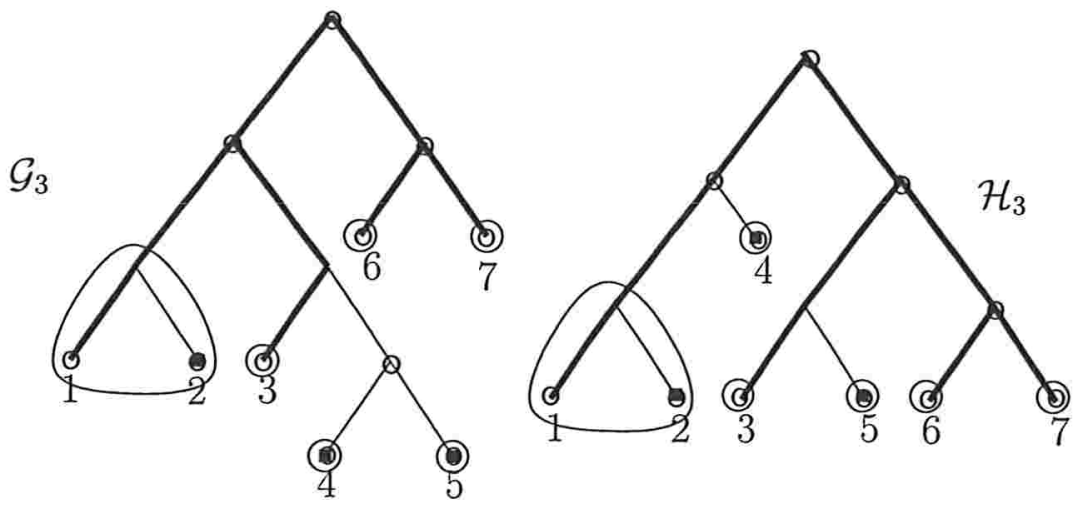

Iteração 3: $\operatorname{Par}(1,3)$, caso $1, k=1$.

Figura 4.6: Aplicação do Algoritmo $\mathcal{A}_{2}$ às árvores $\mathcal{T}$ e $\mathcal{U}$ da Figura 3.3: iterações 1 a 3. 
Capítulo 4. Algoritmos de aproximação para árvores com grau no máximo 249
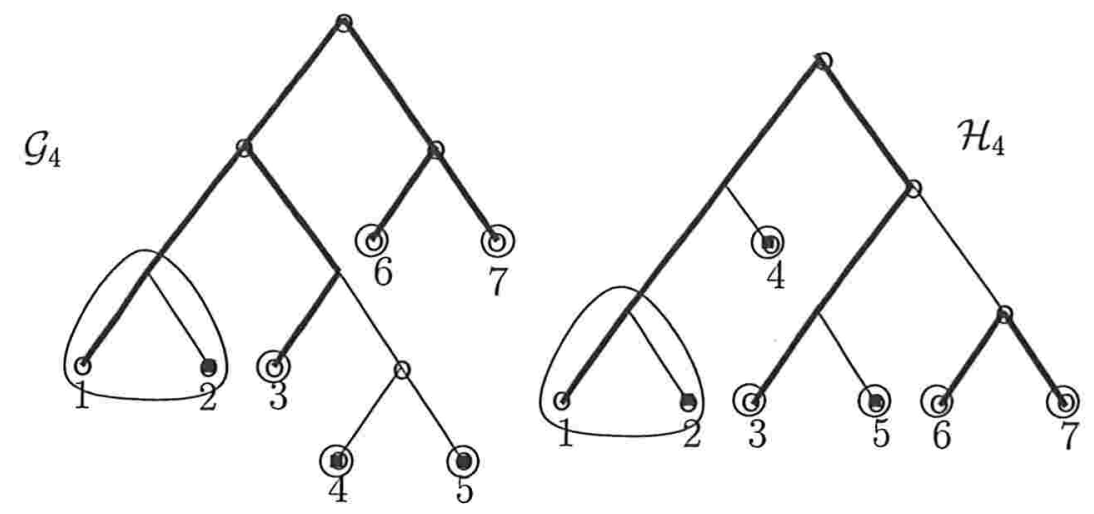

Iteração 4: Par $(1,3)$, caso 5.
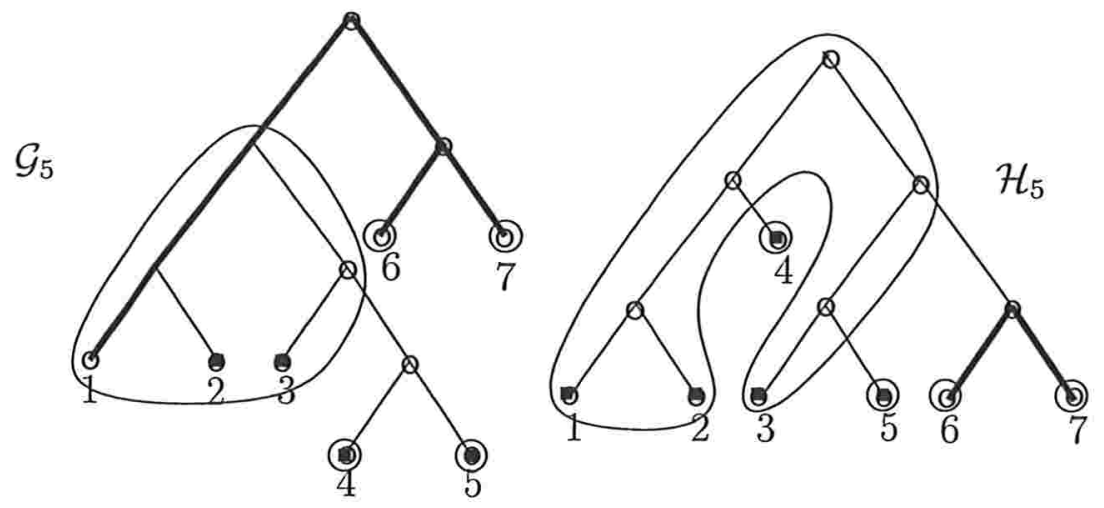

Iteração 5: Par $(6,7)$, caso 5 .
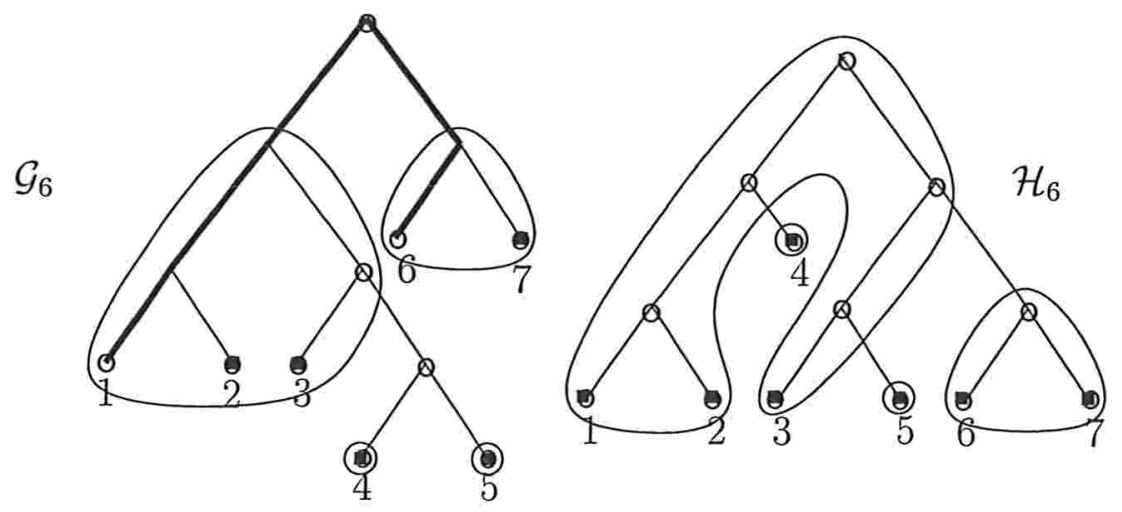

Iteração 6: Par $(1,6)$, caso 4.

Figura 4.7: Continuação da aplicação do Algoritmo $\mathcal{A}_{2}$ : iterações 4 a 6 . 

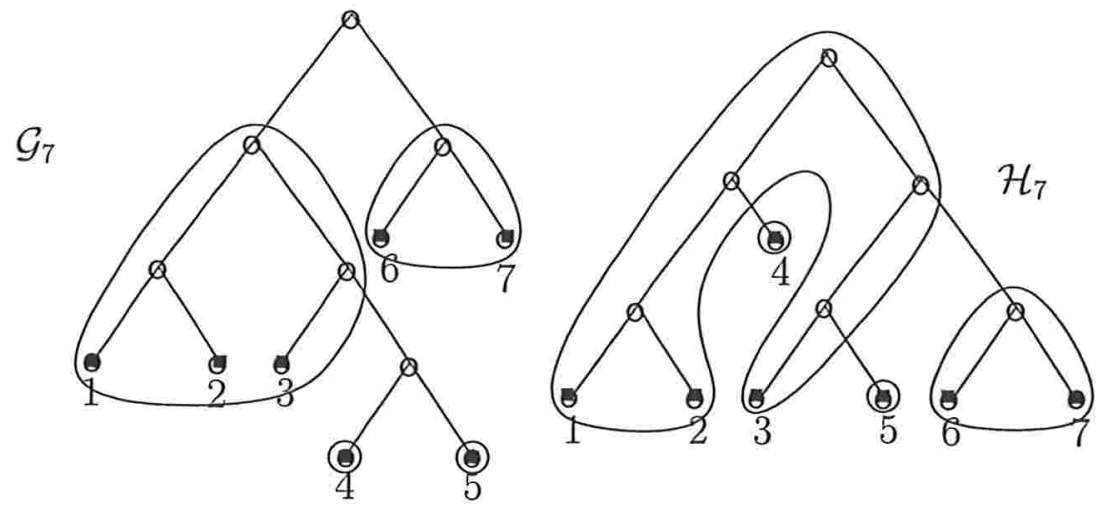

Última iteração: $\mathcal{G}_{7}$ é trivial.

Figura 4.8: Continuação da aplicação do Algoritmo $\mathcal{A}_{2}$ : iteração 7.

os invariantes (1), (2) e (3) podem ser facilmente provados a partir das respectivas hipóteses de indução por inspeção nas definições dos casos e das transações. Observe que $\left|\mathcal{S}_{i}^{j}\right|=\left|\mathcal{S}_{i}^{j-1}\right|$ se $h_{i}^{j-1}$ é um corte, e $\left|\mathcal{S}_{i}^{j}\right|=\left|\mathcal{S}_{i}^{j-1}\right|-1$ se $h_{i}^{j-1}$ é um encolhimento.

Examinando a Tabela 4.1, podemos verificar que o algoritmo $\mathcal{A}_{\bar{k}}$ pára, pois a cada iteração pelo menos um arco de $\mathcal{G}_{i}$ ou $\mathcal{H}_{i}$ é removido. O algoritmo pára quando $\mathcal{G}_{i}$ não tem mais arcos. Neste caso, pelo invariante (2) do Lema $4.3, \mathcal{H}_{i}$ também não tem mais arcos, sendo portanto trivial.

De acordo com o invariante (c), no início da última iteração $\bar{\imath}$ de $\mathcal{A}_{\bar{k}}$, a floresta de concordância $\mathcal{U} \mid \mathcal{S}_{\bar{\imath}}$ de $\mathcal{T}$ e $\mathcal{U}$ tem tamanho igual a $\left|S_{\mathcal{U}}\right|$ menos o número de operações de encolhimento feitas a partir de $\mathcal{U}$. Mas o número total de eliminações (cortes mais encolhimentos) feitos a partir de $\mathcal{U}$ é exatamente $\left|S_{\mathcal{U}}\right|-1$, de acordo com o Lema 4.1. Portanto $|\mathcal{U}| \mathcal{S}_{\bar{\imath}} \mid$ é igual ao número de operações de corte executadas a partir de $\mathcal{U}$ mais 1, que é o número devolvido na linha (9) do método básico (Figura 4.5).

\subsubsection{Complexidade computacional}

Não é difícil concluir que o Algoritmo $\mathcal{A}_{\bar{k}}$ pode ser implementado em tempo quadrático no número $n$ de folhas das árvores filogenéticas $\mathcal{T}$ e $\mathcal{U}$. Seja $m$ o número de arcos nas árvores $\mathcal{T}$ e $\mathcal{U}$.

Cada iteração $i$ do Algoritmo $\mathcal{A}_{\bar{k}}$ pode ser dividida em três fases: teste de parada (linha 4 na Figura 4.5), determinação do caso (linha 5) e aplicação da transação correspondente (linha 6). O teste de parada consiste em localizar um par de folhas irmãs $f_{\mathcal{G}_{i}}(a)$ e $f_{\mathcal{G}_{i}}(b)$ em $\mathcal{G}_{i}$. Isso pode ser feito em tempo $O(n)$ se armazenamos as folhas de $\mathcal{G}_{i}$ em um vetor na pré-ordem induzida por uma ordenação dos descendentes 
imediatos dos nós internos da topologia de $\mathcal{G}_{i}$. Neste caso, apenas pares de folhas vizinhas neste vetor podem ser irmãs em $\mathcal{G}_{i}$. A determinação do caso consiste em localizarmos o eixo $(a, b)$ em $\mathcal{H}_{i}$ e, caso ele exista, determinar o número de hastes deste eixo. Isso pode ser feito em tempo $O(m)$. Finalmente, as transações podem ser feitas em tempo $O(m)$ para cada $\bar{k}$ (veja as Seções 5.2 e 5.3). Portanto, cada iteração pode ser executada em tempo $O(n)+O(m)+O(m)$.

Um exame da Tabela 4.1 mostra que a cada iteração o número de arcos de pelo menos uma das florestas $\mathcal{G}_{i}$ e $\mathcal{H}_{i}$ diminui ao menos de um. Como $\mathcal{T}$ e $\mathcal{U}$ não admitem nós internos com grau 1, então $m=O(n)$. Portanto o número de iterações é $O(n)$, donde segue que o Algoritmo $\mathcal{A}_{\bar{k}}$ tem complexidade $O\left(n^{2}\right)$.

\subsection{Razão de aproximação do algoritmo $\mathcal{A}_{\bar{k}}$}

\subsubsection{Limitantes inferiores}

Inicialmente, vamos mostrar que os Algoritmos $\mathcal{A}_{1}$ e $\mathcal{A}_{2}$ têm razão de aproximação pelo menos 4 e 3 , respectivamente. Vamos fazer isso exibindo duas famílias de instâncias do problema MAF-2 tal que:

- Para todo $\epsilon>0$, a primeira família apresentada possui uma instância $\mathcal{T}, \mathcal{U}$ tal que:

$$
\frac{m_{\mathrm{MAF}-2}\left(\mathcal{T}, \mathcal{U}, \mathcal{A}_{1}(\mathcal{T}, \mathcal{U})\right)}{m_{\mathrm{MAF}-2}^{*}(\mathcal{T}, \mathcal{U})}>(4-\epsilon)
$$

- Para todo $\epsilon>0$, a segunda família apresentada possui uma instância $\mathcal{T}, \mathcal{U}$ tal que:

$$
\frac{m_{\mathrm{MAF}-2}\left(\mathcal{T}, \mathcal{U}, \mathcal{A}_{2}(\mathcal{T}, \mathcal{U})\right)}{m_{\mathrm{MAF}-2}^{*}(\mathcal{T}, \mathcal{U})}>(3-\epsilon){ }^{1}
$$

Em seguida, vamos generalizar estes resultados para obter, para cada $\bar{k} \geq 1$, um limitante inferior para a razão de aproximação do algoritmo $\mathcal{A}_{\bar{k}}$.

Para o Algoritmo $\mathcal{A}_{1}$, considere a família exemplificada pela instância mostrada na parte inferior da Figura 4.9. De acordo com essa figura, podemos identificar duas seções distintas na árvore $\mathcal{U}$. Vamos chamar estas seções de seção inferior e seção superior de $\mathcal{U}$. A seção inferior de $\mathcal{U}$ compõe-se de $m \geq 1$ configurações básicas $C_{1}, \ldots, C_{m}$ (veja a parte superior esquerda da Figura 4.9) conectadas umas às outras

\footnotetext{
${ }^{1}$ Nas duas expressões acima envolvendo a medida $m_{\mathrm{MAF}-2}$ das soluções viáveis do problema MAF-2, estamos omitindo alguns pares de parênteses, para maior clareza. De acordo com a notação definida np Capítulo 2, escreveríamos $m_{\mathrm{MAF}-2}^{*}((\mathcal{T}, \mathcal{U}))$ em lugar de $m_{\mathrm{MAF}-2}^{*}(\mathcal{T}, \mathcal{U})$ e $m_{\mathrm{MAF}-2}\left((\mathcal{T}, \mathcal{U}), \mathcal{A}_{1}((\mathcal{T}, \mathcal{U}))\right)$ em lugar de $m_{\mathrm{MAF}-2}\left(\mathcal{T}, \mathcal{U}, \mathcal{A}_{1}(\mathcal{T}, \mathcal{U})\right)$.
} 
através dos nós $x_{i}$ e $y_{i}, 1<i \leq m$. Para $i=1, \ldots, m-1$, as configurações $C_{i}$ e $C_{i+1}$ são conectadas identificando-se os nós $x_{i+1}$ de $C_{i+1}$ e $y_{i}$ de $C_{i}$, de modo que elas aparecem em $\mathcal{U}$ em ordem crescente $\left(C_{1}, \ldots, C_{m}\right)$ de baixo para cima. A seção superior de $\mathcal{U}$ por sua vez é composta de uma cadeia de $m+1$ folhas (veja a parte superior direita da Figura 4.9), e conecta-se à seção inferior através da identificação dos nós $y_{m}$ e $z$.

A árvore $\mathcal{T}$ é construída de modo a obrigar o Algoritmo $\mathcal{A}_{1}$ a executar uma seqüência de $m$ iterações caso 2 (cortando em $\mathcal{U}$ as folhas $3 i-2$ e $3 i$ para $i=$ $1, \ldots, m$ ), em seguida executar mais $m-1$ iterações caso 2 (cortando a folha $3 i-1$ para $i=1, \ldots, m-1$, mais as folhas $3 m+1, \ldots, 4 m-1$ ), e finalmente executar uma iteração caso 1 (cortando em $\mathcal{U}$ a folha $x_{1}$ ), uma iteração caso 5 e uma iteração caso 4 (deixando as folhas $3 m-1$ e $4 m$ num mesmo componente).

A instância mostrada na Figura 4.9 exemplifica esta construção para $m=9$. Na Figura 4.10 vemos alguns estados intermediários desta instância durante uma execução do Algoritmo $\mathcal{A}_{1}$. Nas primeiras $m$ iterações o algoritmo corta em $\mathcal{U}$ as folhas $1,3,4,6, \ldots, 25,27$ (Figura 4.10, esquerda), nas $m-1$ iterações seguintes são cortadas as folhas $2,28,5,29, \ldots, 23,35$ (Figura 4.10, direita), e nas três últimas iterações corta-se a folha 0 em $\mathcal{U}$, encolhe-se as folhas 26 e 36 em $\mathcal{T}$ e $\mathcal{U}$ e separa-se a folha resultante deste encolhimento da folha $0 \mathrm{em} \mathcal{T}$.

Para cada membro desta família de instâncias o algoritmo $\mathcal{A}_{1}$ produz uma floresta de concordância com $2 m+2(m-1)+1+1=4 m$ componentes, enquanto que existe uma solução viável de tamanho $m+2$, indicada em negrito na Figura 4.9. Portanto, se tomarmos $m>\max (8 / \epsilon-2,0)$, teremos:

$$
\frac{m_{\mathrm{MAF}-2}\left(\mathcal{T}, \mathcal{U}, \mathcal{A}_{1}(\mathcal{T}, \mathcal{U})\right)}{m_{\mathrm{MAF}-2}^{*}(\mathcal{T}, \mathcal{U})} \geq \frac{4 m}{m+2}=4-\frac{8}{m+2}>4-\epsilon .
$$

Para o Algoritmo $\mathcal{A}_{2}$, considere a família de instâncias construída de modo similar à família que apresentamos para o Algoritmo $\mathcal{A}_{1}$, porém usando a configuração básica mostrada na parte superior da Figura 4.11 em lugar da configuração mostrada na Figura 4.9 na construção da seção inferior da árvore $\mathcal{U}$, e empregando $2 m+1$ folhas (incluindo o nó $z$ ) na cadeia que forma a seção superior de $\mathcal{U}$. A parte inferior da Figura 4.11 exibe um exemplo de instância desta família para $m=9$. De modo similar à família apresentada anteriormente, a árvore $\mathcal{T}$ induz o Algoritmo $\mathcal{A}_{2}$ a executar $m$ iterações caso 2 (cortando as folhas 1, 4, 5, 8, ., 33, 36 no exemplo da Figura 4.11), em seguida mais $2(m-1)$ iterações caso 2 (cortando com isso as folhas $2,37,3,38$, $\ldots, 31,52$ no exemplo), e finalmente mais cinco iterações: uma caso 1 (em que são cortados os nós 35 e 0), duas caso 5 e duas caso 4 .

Para cada membro desta família de instâncias, o Algoritmo $\mathcal{A}_{2}$ produz uma floresta de concordância com $2 m+4(m-1)+2+1=6 m-1$ componentes, enquanto que existe uma solução viável de tamanho $2 m+2$, mostrada em negrito na Figura 4.11. 
Capítulo 4. Algoritmos de aproximação para árvores com grau no máximo 253
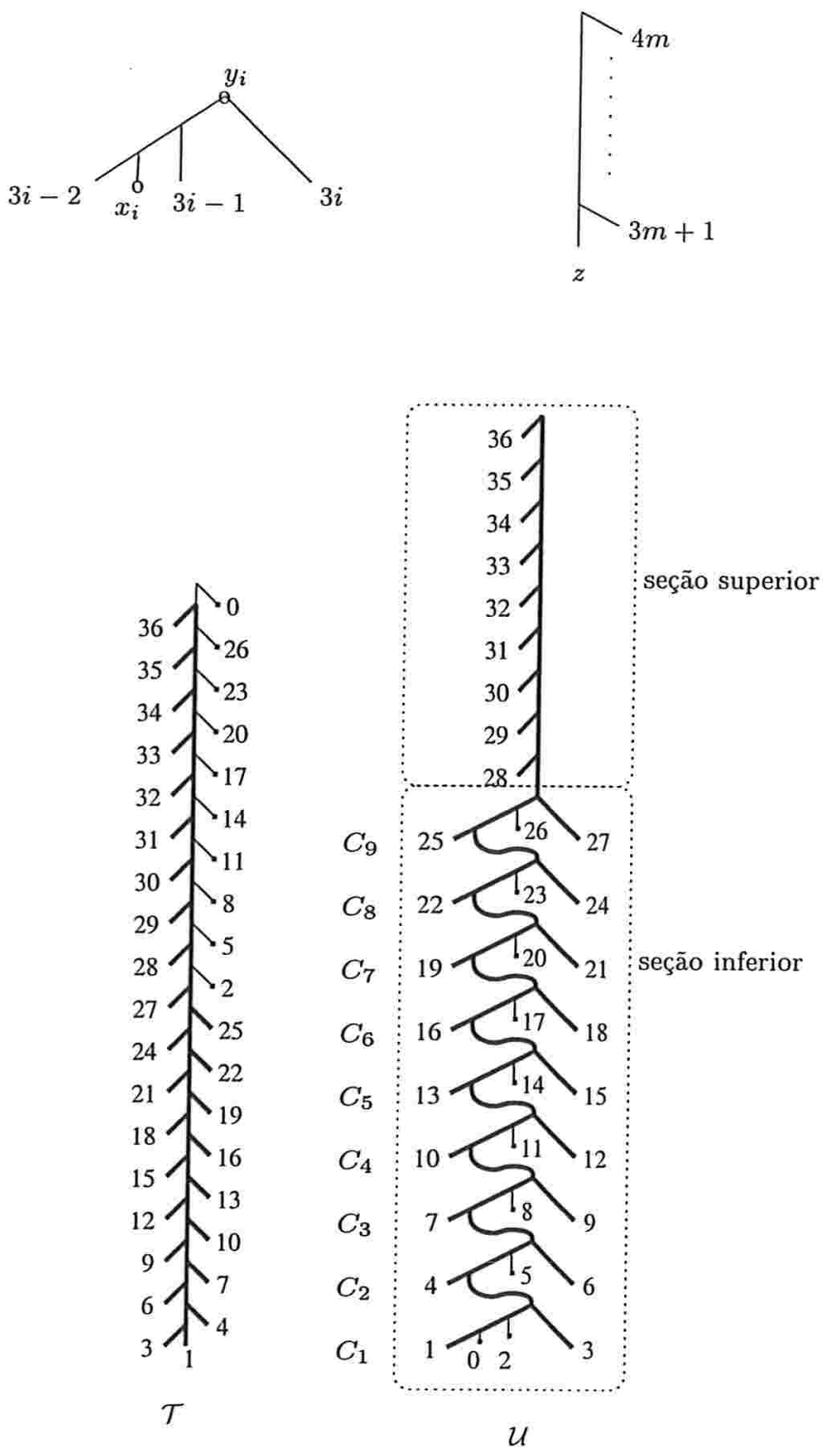

Figura 4.9: Instância de uma família que mostra que o Algoritmo $\mathcal{A}_{1}$ tem razão de desempenho pelo menos 4 . 
Capítulo 4. Algoritmos de aproximação para árvores com grau no máximo 254
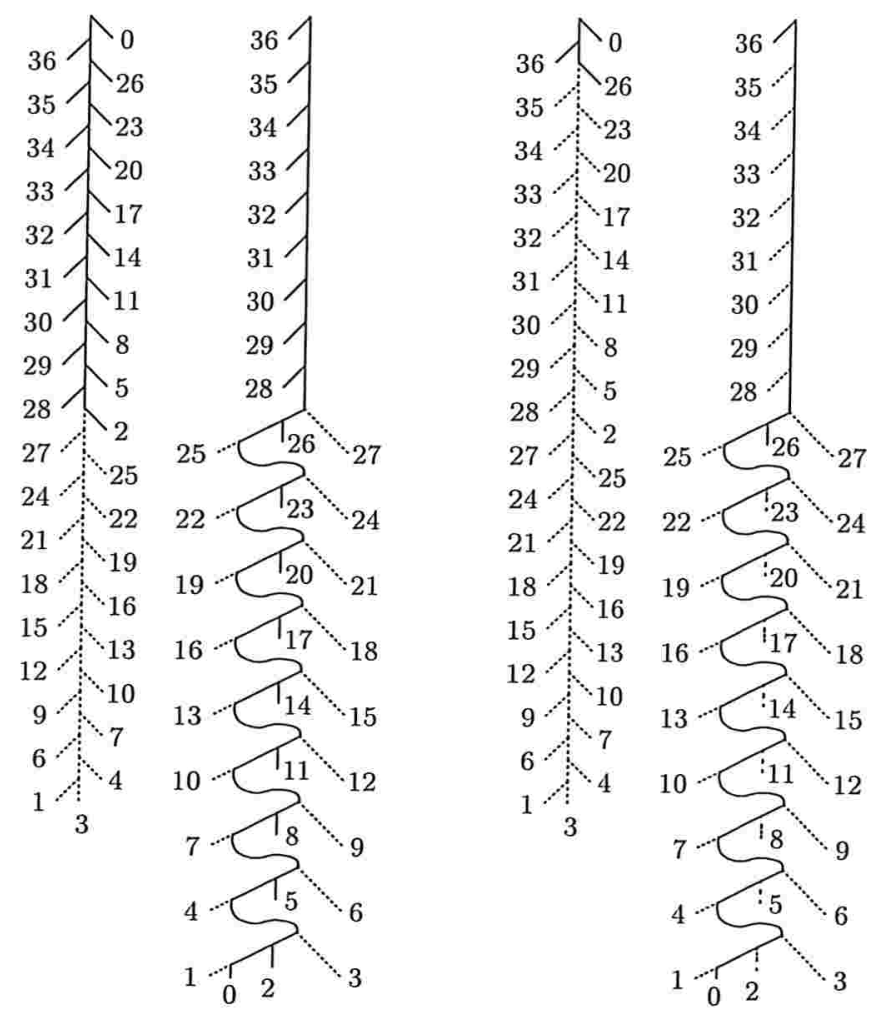

Figura 4.10: Exemplo da Figura 4.9 depois das $m$ primeiras iterações (esquerda) e depois das $m-1$ iterações seguintes (direita). 
Portanto, para $m>\max (7 / 2 \epsilon-1,0)$, temos:

$$
\frac{m_{\mathrm{MAF}-2}\left(\mathcal{T}, \mathcal{U}, \mathcal{A}_{1}(\mathcal{T}, \mathcal{U})\right)}{m_{\mathrm{MAF}-2}^{*}(\mathcal{T}, \mathcal{U})} \geq \frac{6 m-1}{2 m+2}=3-\frac{7}{2 m+2}>3-\epsilon
$$

É possível obter limitantes inferiores para todos os algoritmos $\mathcal{A}_{\bar{k}}, \bar{k} \geq 1$, generalizando-se os exemplos exibidos acima. A construção das famílias de instâncias segue os mesmos princípios gerais vistos acima, sendo necessário alterar apenas os seguintes detalhes: a configuração básica utilizada na construção da seção inferior de $\mathcal{U}$ é como mostra a parte superior da Figura 4.12; e a seção superior de $\mathcal{U}$ deve possuir $\bar{k} m+1$ folhas. A parte inferior da Figura 4.12 esquematiza uma instância desta família com $m \geq 1$ configurações para o Algoritmo $\mathcal{A}_{\bar{k}}$.

Em cada instância desta família, o Algoritmo $\mathcal{A}_{\bar{k}}$ executa inicialmente $m$ iterações caso 2 (cortando as folhas $i(\bar{k}+2)$ e $i(\bar{k}+2)-(\bar{k}+1)$ para $i=1, \ldots, m)$, em seguida executa mais $\bar{k}(m-1)$ iterações caso 2 (cortando as folhas $m(\bar{k}+2)+\bar{k} i+j$ e $(i+1)(\bar{k}+2)-(\bar{k}-j+1)$ para $i=0, \ldots, m-2$ e $j=1, \ldots, \bar{k})$, e finalmente executa mais uma iteração caso 1 (cortando as folhas $0, m(\bar{k}+2)-\bar{k}+1, \ldots, m(\bar{k}+2)-1), \bar{k}$ iterações caso 5 e $\bar{k}$ iterações caso 4 . Portanto o algoritmo produz para esta instância uma solução de tamanho $2 m+2 \bar{k}(m-1)+\bar{k}+1$, enquanto que existe uma solução de tamanho $\bar{k} m+2$, marcada em negrito na Figura 4.12 .

Portanto, se tomarmos

$$
m>\max \left(\frac{\bar{k}^{2}+3 \bar{k}+4}{\bar{k}^{2} \epsilon}-\frac{2}{\bar{k}}, 0\right),
$$

teremos:

$$
\begin{aligned}
\frac{m_{\mathrm{MAF}-2}\left(\mathcal{T}, \mathcal{U}, \mathcal{A}_{k}(\mathcal{T}, \mathcal{U})\right)}{m_{\mathrm{MAF}-2}^{*}(\mathcal{T}, \mathcal{U})} & \geq \frac{2 m+2 \bar{k}(m-1)+\bar{k}+1}{\bar{k} m+2} \\
& =\frac{2 \bar{k} m+2 m-\bar{k}+1}{\bar{k} m+2} \\
& =\frac{2 \bar{k} m+4}{\bar{k} m+2}+\frac{2 m-\bar{k}-3}{\bar{k} m+2} \\
& =2+\frac{2 m-\bar{k}-3}{\bar{k} m+2} \\
& =2+\frac{2 m+\frac{4}{\bar{k}}}{\bar{k} m+\frac{\bar{k}}{\bar{k}} m+3+\frac{4}{\bar{k}}} \\
& =2+\frac{2}{\bar{k}}-\frac{\bar{k}+3 \bar{k}+4}{\bar{k}(\bar{k} m+2)} \\
& >2+\frac{2}{\bar{k}}-\epsilon .
\end{aligned}
$$

Com isso, mostramos que a razão de aproximação do Algoritmo $\mathcal{A}_{\bar{k}}$ não pode ser inferior a $2+2 / \bar{k}$ para todo $\bar{k}$. 
Capítulo 4. Algoritmos de aproximação para árvores com grau no máximo 256
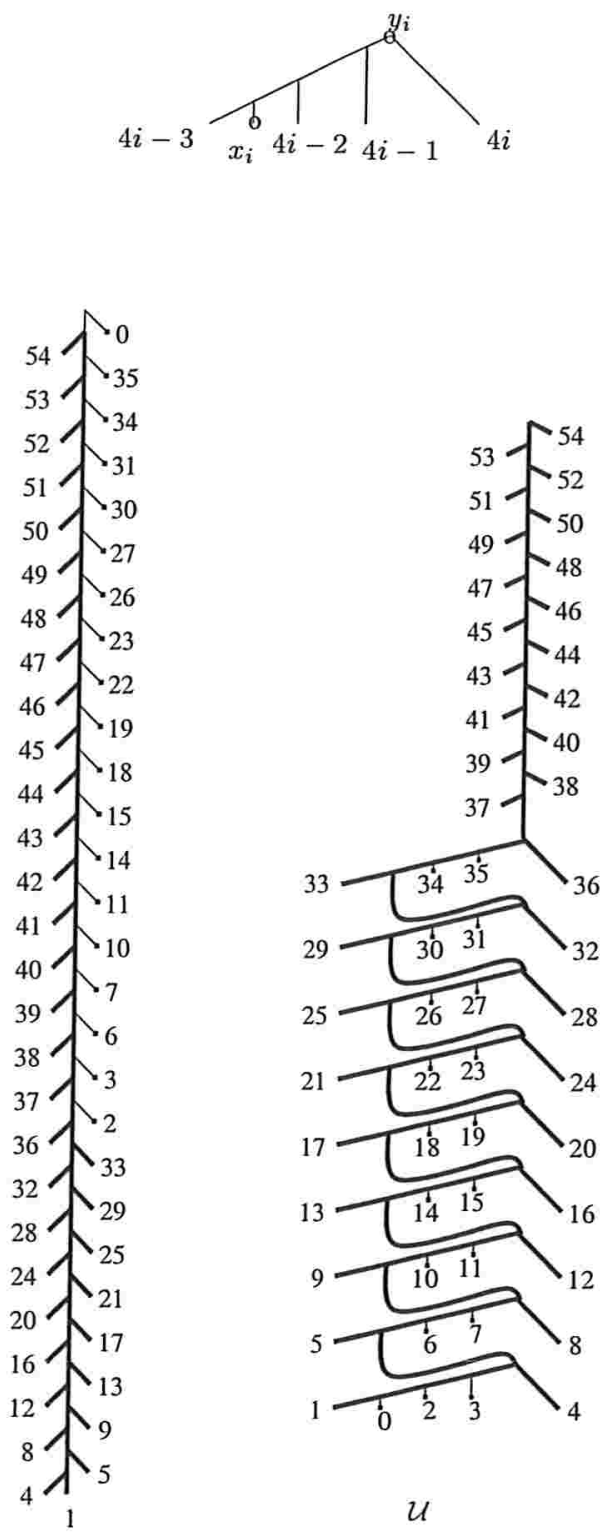

$\mathcal{T}$

Figura 4.11: Instância de uma família que mostra que o Algoritmo $\mathcal{A}_{2}$ tem razão de desempenho pelo menos 3 . 

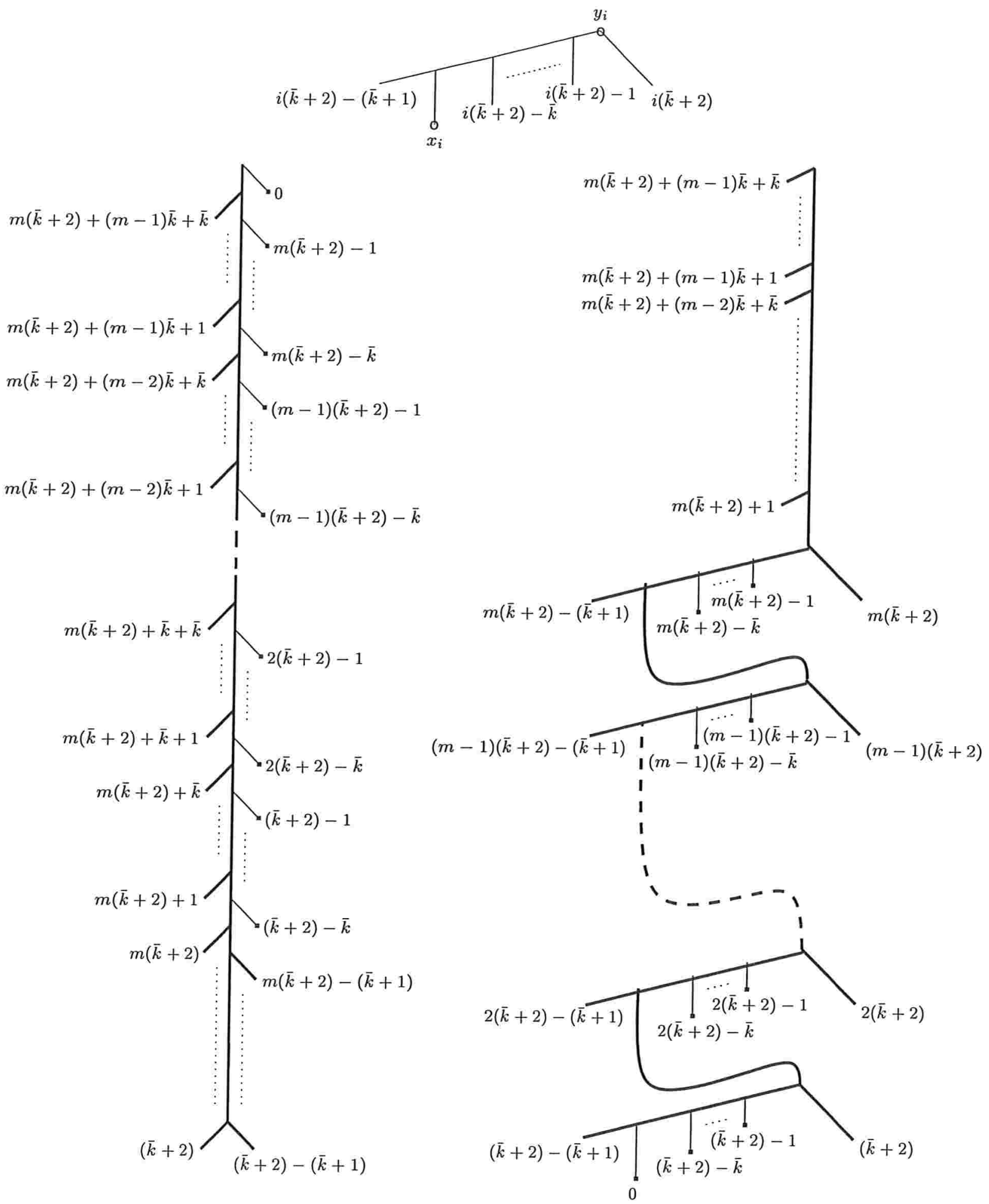

Figura 4.12: Generalização dos exemplos anteriores para o Algoritmo $\mathcal{A}_{\bar{k}}$. 


\subsubsection{Restrições completas e o método básico}

A análise da razão de aproximação do Algoritmo $\mathcal{A}_{\bar{k}}$ baseia-se na idéia de comparar os arcos cortados pelo Algoritmo $\mathcal{A}_{\bar{k}}$ com os arcos de uma floresta de concordância ótima das árvores $\mathcal{T}$ e $\mathcal{U}$ fixada no início das iterações do algoritmo. Para fazer essa comparação, vamos usar o seguinte artifício.

Fixamos uma floresta de concordância ótima $\mathcal{F}$ das árvores $\mathcal{T}$ e $\mathcal{U}$ dadas na entrada e consideramos a restrição $\mathcal{U} \mid \mathcal{S}_{\mathcal{F}}$ induzida por $\mathcal{S}_{\mathcal{F}}$ em $\mathcal{U}$. A análise é baseada na comparação entre os arcos de $\mathcal{U}$ cortados pelo algoritmo e os arcos de ligação relativos à restrição $\mathcal{U} \mid \mathcal{S}_{\mathcal{F}}$. Para fazer essa comparação, começamos com as restrições completas induzidas pela floresta $\mathcal{F}$ sobre $\mathcal{G}_{1}$ e $\mathcal{H}_{1}$ no início da primeira iteração. Depois, acompanhando as operações efetuadas pelo algoritmo $\mathcal{A}_{\bar{k}}$ nos sucessivos $\mathcal{G}_{i}$ e $\mathcal{H}_{i}$, vamos fazendo transformações nas sucessivas restrições completas $\mathcal{G}_{i}^{j}$ e $\mathcal{H}_{i}^{j}$ que vão sendo obtidas. Tais restrições preservam os arcos de ligação definidos inicialmente em $\mathcal{T}$ e $\mathcal{U}$ por $\mathcal{F}$

As Figuras 4.13, 4.14 e 4.15 mostram a evolução de uma floresta de concordância ótima nas restrições $\mathcal{G}_{i}$ e $\mathcal{H}_{i}$ do exemplo das Figuras 4.6 a 4.8 à medida que as iterações do algoritmo se sucedem. Na figura, $\mathcal{G}_{i}$ e $\mathcal{H}_{i}$ são indicadas pelos arcos escuros e os componentes da floresta de concordância $\mathcal{F}$ são indicados por linhas curvas. Note que nas iterações 2 e 3 há arcos de ligação sendo cortados.

Pelo Lema 4.2 (fazendo $\mathcal{H}$ referir-se a $\mathcal{U}$ e $\mathcal{S}$ referir-se à floresta de concordância $\mathcal{F}$ ) temos que o número destes arcos de ligação que são eliminados ao longo da execução do algoritmo é exatamente $|\mathcal{F}|-1$. Por outro lado, vimos na Seção 4.3.2 que existe uma floresta de concordância de $\mathcal{T}$ e $\mathcal{U}$ cujo tamanho é exatamente o número de arcos cortados pelo algoritmo mais um.

Assim, se $t$ é o valor devolvido pelo Algoritmo $\mathcal{A}_{\bar{k}}$ quando executado com entradas $\mathcal{T}$ e $\mathcal{U}$, então pelo argumento que acabamos de expor, ao longo da execução o algoritmo corta $t-1$ arcos e elimina $|\mathcal{F}|-1$ arcos de ligação com relação à restrição $\mathcal{U} \mid \mathcal{S}_{\mathcal{F}}$.

O objetivo da análise é mostrar que o número total de arcos de $\mathcal{U}$ cortados pelo Algoritmo $\mathcal{A}_{\bar{k}}$ é menor ou igual a um múltiplo do número de arcos de ligação eliminados. Uma vez que isso esteja estabelecido, temos que $t$ é limitado por um múltiplo de $|\mathcal{F}|$, conforme desejamos mostrar.

Há alguns itens que precisam ser detalhados para que este argumento possa ser aplicado. Em primeiro lugar, precisamos mostrar como obter restrições completas de $\mathcal{H}_{i}^{j}$ a partir de $\mathcal{U} \mid \mathcal{S}_{\mathcal{F}}$ que preservam os arcos de ligação definidos inicialmente por $\mathcal{U} \mid \mathcal{S}_{\mathcal{F}}$ em $\mathcal{U}$. E em segundo lugar, precisamos introduzir algumas propriedades do método básico que nos permitam relacionar os números de arcos comuns cortados e arcos de ligação eliminados durante cada iteração de $\mathcal{A}_{\bar{k}}$.

Definimos a seguir, para $i \geq 1$, as restrições completas de $\mathcal{H}_{i}^{j}$ obtidas a partir da 
Capítulo 4. Algoritmos de aproximação para árvores com grau no máximo 259
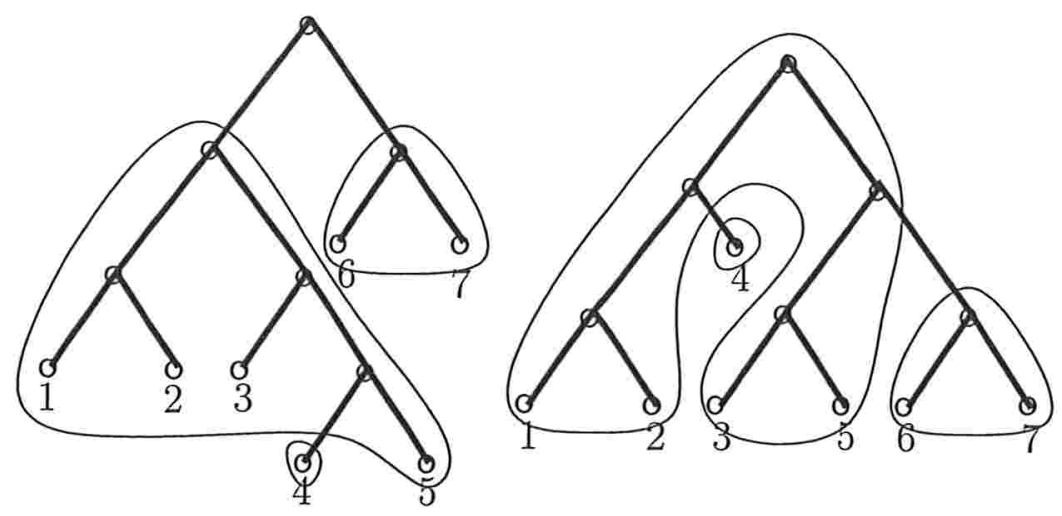

Iteração 1: Par $(1,2)$, caso 5.
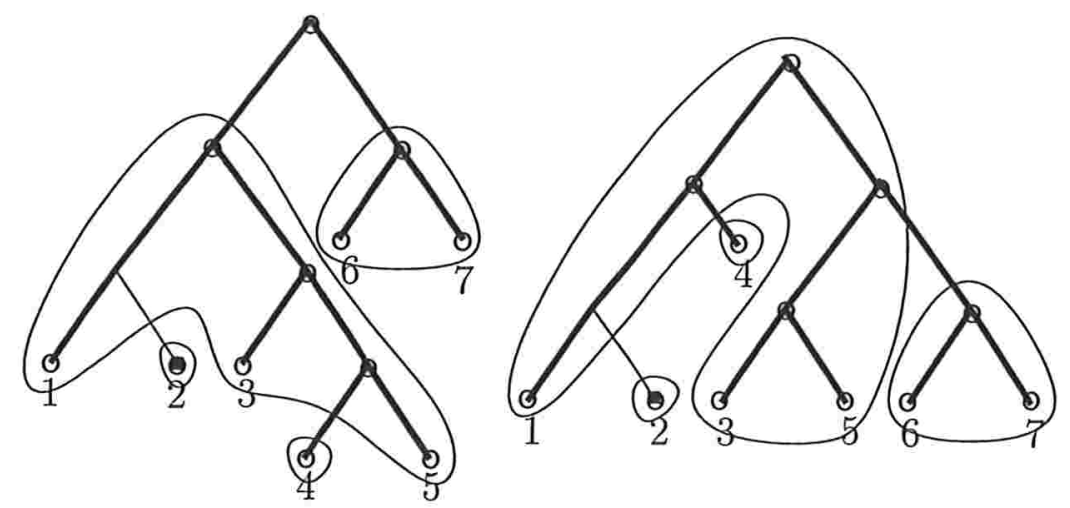

Iteração 2: Par $(4,5)$, caso 2 .
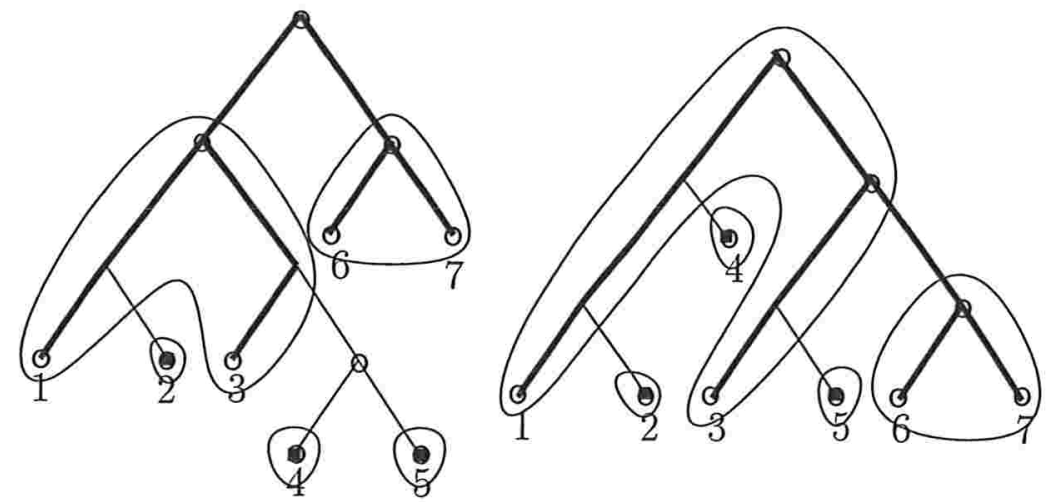

Iteração 3: Par $(1,3)$, caso 1 .

Figura 4.13: Restrições $\mathcal{G}_{i}^{\prime}$ e $\mathcal{H}_{i}^{\prime}$ na execução do Algoritmo $\mathcal{A}_{2}$ correspondentes às iterações 1 a 3 na Figura 4.6. 
Capítulo 4. Algoritmos de aproximação para árvores com grau no máximo 260

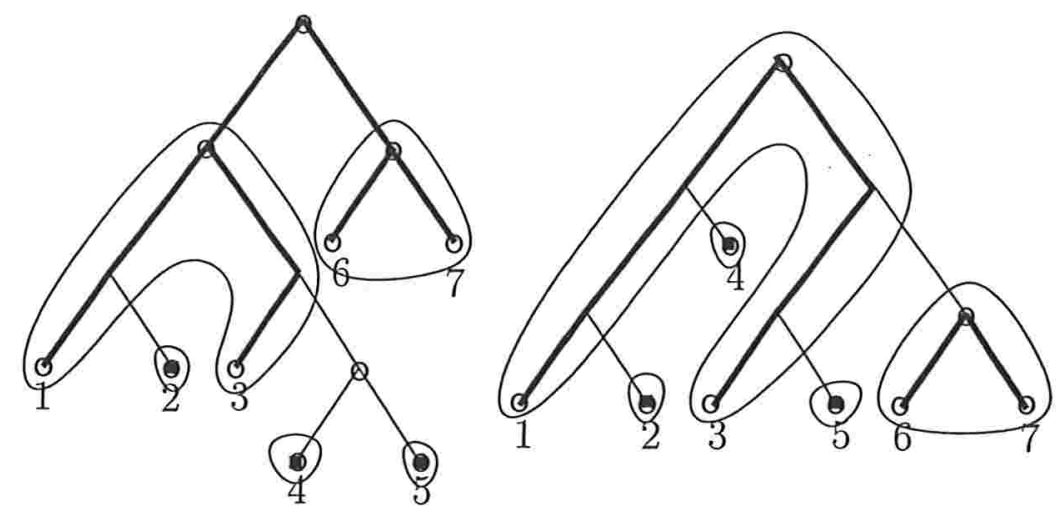

Iteração 4: Par $(1,3)$, caso 5.

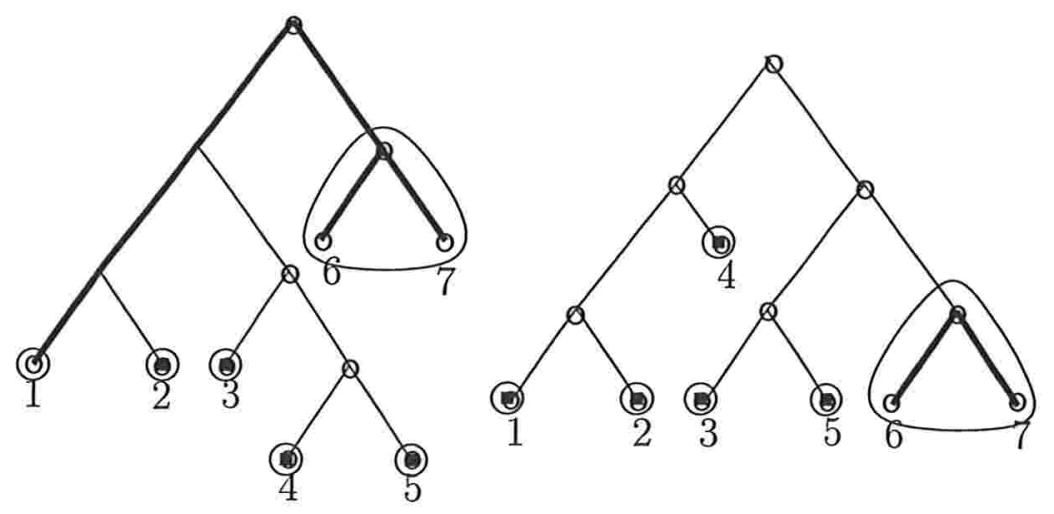

Iteração 5: Par $(6,7)$, caso 5 .
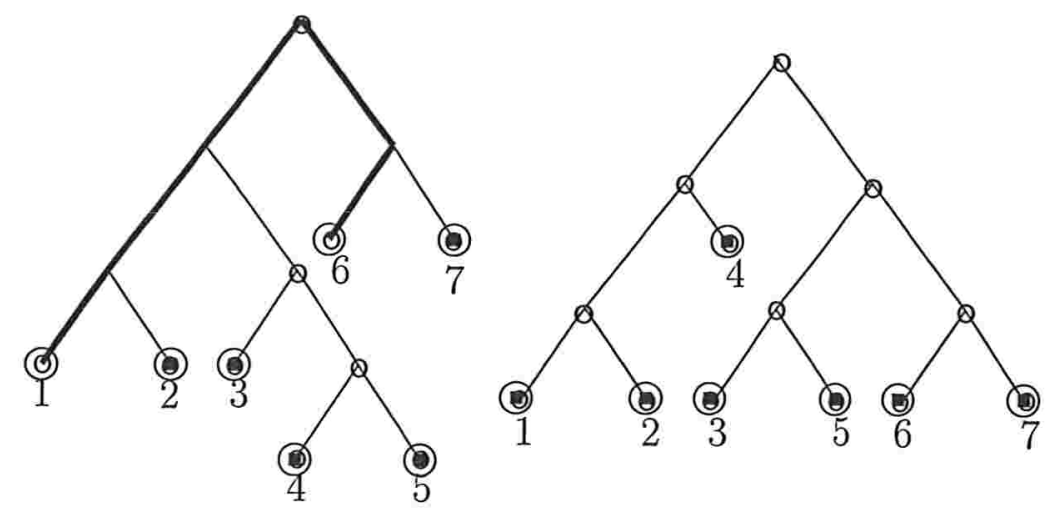

Iteração 6: Par $(1,6)$, caso 4.

Figura 4.14: Restrições $\mathcal{G}_{i}^{\prime}$ e $\mathcal{H}_{i}^{\prime}$ na execução do Algoritmo $\mathcal{A}_{2}$ correspondentes às iterações 4 a 6 na Figura 4.7 . 


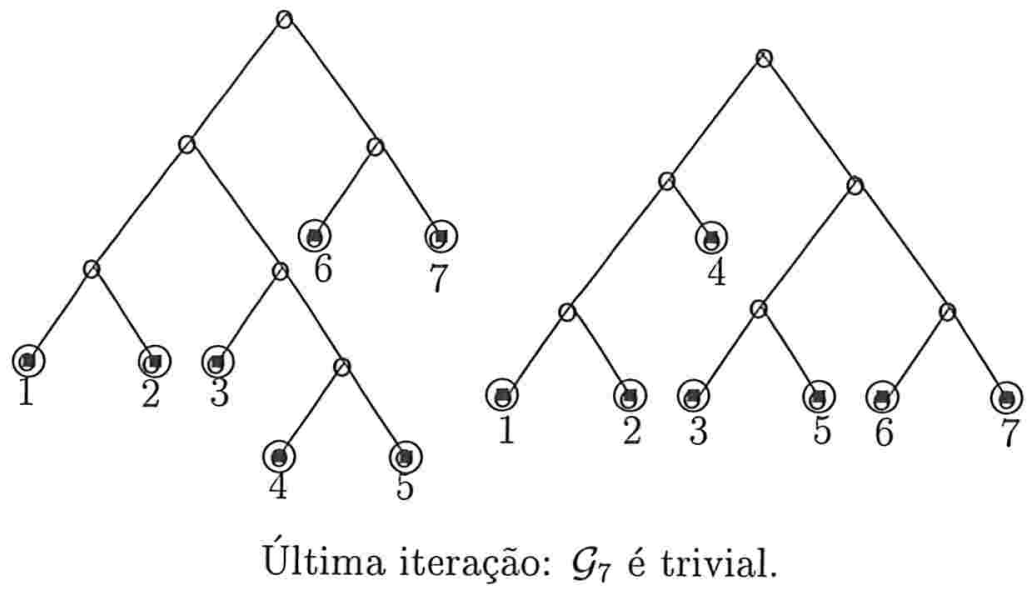

Figura 4.15: Restrições $\mathcal{G}_{i}^{\prime}$ e $\mathcal{H}_{i}^{\prime}$ na execução do Algoritmo $\mathcal{A}_{2}$ correspondentes à iteração 7 na Figura 4.8.

restrição $\mathcal{U} \mid \mathcal{S}_{\mathcal{F}}$.

Seja $\mathcal{H}_{1}^{\prime}$ a restrição completa $\mathcal{U} \mid \mathcal{S}_{\mathcal{F}}$. Para $i \geq 2$, seja $\mathcal{H}_{i}^{\prime}$ a restrição que obtemos de $\mathcal{H}_{i-1}^{\prime}$ após executar a partir desta a seqüência de operações que definimos a seguir.

Para $i \geq 1$, seja $\mathcal{H}_{i}^{\prime \mathbf{1}}$ a restrição $\mathcal{H}_{i}$, e para $j=2, \ldots, \bar{\jmath}_{i}+1$, seja $\mathcal{H}^{\prime j}{ }_{i}$ a restrição $\mathcal{H}_{i}^{j-1}$ se o arco $e$ de $\mathcal{H}_{i}^{j-1}$ eliminado quando o algoritmo executa a operação $h_{i}^{j-1}$ é de ligação com respeito a $\mathcal{H}_{i}^{\prime j-1}$; caso contrário, seja $\mathcal{H}_{i}^{\prime j}:=\mathcal{H}_{i}^{\prime j-1} \mid \mathcal{S}$, onde $\mathcal{S}$ é uma família de subconjuntos de $\mathcal{S}_{\mathcal{H}_{i}^{j}}$ tal que $|\mathcal{S}|=\left|\mathcal{S}_{\mathcal{H}_{i}^{j-1}}\right|+1, \mathcal{S}$ define uma restrição completa em $\mathcal{H}_{i}^{j}$ e $\mathcal{S}$ preserva todos os arcos de ligação definidos por $\mathcal{H}_{i}^{\prime j-1}$ em $\mathcal{H}_{i}^{j-1}$.

Podemos verificar facilmente, a partir desta definição, que $\mathcal{H}_{i}^{\prime j}$ é o resultado da operação $\operatorname{Elim}\left(\mathcal{H}_{i}^{\prime j-1}, l(e)\right)$ quando e não é um arco de ligação com respeito a $\mathcal{H}_{i}^{\prime j-1}$.

$\mathrm{O}$ invariante dado no lema abaixo estabelece que $\mathcal{H}_{i}^{\prime j}$ de fato satisfaz as propriedades listadas acima.

Lema 4.4 Para cada iteração $i \geq 1$ e $j=1, \ldots, \bar{j}_{i}+1$, temos que $\mathcal{H}_{i}^{\prime j}$ é uma restrição completa de $\mathcal{H}_{i}^{j}$. Além disso, ela é isomorfa a uma restrição completa de $\mathcal{G}_{i}^{j}$ e preserva os arcos de ligação definidos por $\mathcal{U} \mid \mathcal{S}_{\mathcal{F}}$ em $\mathcal{U}$ que ainda não foram eliminados até essa iteração.

Prova. Provamos este lema por indução nas seqüências de operações feitas pelo algoritmo a partir de $\mathcal{U}$.

A base da indução é válida pois $\mathcal{H}_{1}^{\prime 1}=\mathcal{U} \mid \mathcal{S}_{\mathcal{F}}$, que é uma floresta de concordância de $\mathcal{G}_{1}^{1}=\mathcal{T}$ e $\mathcal{H}_{1}^{1}=\mathcal{U}$.

Sejam $i \geq 1$ e $j=2, \ldots, \bar{\jmath}_{i}+1$. No caso em que o arco e eliminado em $\mathcal{H}_{i}^{j-1}$ 
pela operação $h_{i}^{j-1}$ é de ligação com relação a $\mathcal{H}_{i}^{\prime j-1}$, temos que $\mathcal{H}_{i}^{\prime j}$ é uma restrição completa de $\mathcal{H}_{i}^{j-1}$ pela definição de $\mathcal{H}_{i}^{\prime j}$ e pela hipótese de indução. Como $\mathcal{H}_{i}^{j}$ é obtida da eliminação do arco e em $\mathcal{H}_{i}^{j-1}$ e esse arco é de ligação com relação a $\mathcal{H}_{i}^{\prime j-1}=\mathcal{H}_{i}^{\prime j}$, então $\mathcal{H}_{i}^{\prime j}$ é uma restrição completa de $\mathcal{H}_{i}^{j}$. Claramente, $\mathcal{H}_{i}^{\prime j}$ preserva os arcos de ligação definidos por $\mathcal{H}_{i}^{\prime j-1}$ em $\mathcal{H}_{i}^{j-1}$ exceto $e$.

No caso em que o arco e não é de ligação com relação a $\mathcal{H}_{i}^{\prime j-1}$, a existência da família $\mathcal{S}$ mencionada na definição de $\mathcal{H}_{i}^{\prime j}$ é garantida pelos Lemas 3.2 e 3.5.

Analisando a Tabela 4.1 e pela hipótese de indução, podemos verificar que $\mathcal{H}_{i}^{\prime j}$ é isomorfa a uma restrição completa de $\mathcal{G}_{i}^{j}$.

Prosseguimos apresentando uma propriedade fundamental dos casos definidos na Seção 4.2 .

Lema 4.5 Sejam $\mathcal{G}$ e $\mathcal{H}$ duas florestas filogenéticas com $S_{\mathcal{G}}=S_{\mathcal{H}}$, e seja $\mathcal{H}^{\prime}$ uma restrição completa de $\mathcal{H}$ isomorfa a uma restrição completa de $\mathcal{G}$. Suponhamos que a e $b \in S_{\mathcal{G}}$ são tais que $f_{\mathcal{G}}(a)$ e $f_{\mathcal{G}}(b)$ são folhas irmãs em $\mathcal{G}$.

1) $S$ e $f_{\mathcal{H}^{\prime}}(a)$ e $f_{\mathcal{H}^{\prime}}(b)$ são folhas isoladas em componentes de $\mathcal{H}^{\prime}$ distintos e $f_{\mathcal{H}}(a)$ e $f_{\mathcal{H}}(b)$ não estão isoladas, então pelo menos um dos arcos que incidem em $f_{\mathcal{H}}(a)$ e $f_{\mathcal{H}}(b)$ é um arco de ligação de $\mathcal{H}$ com respeito a $\mathcal{S}_{\mathcal{H}^{\prime}}$.

2) Se $f_{\mathcal{H}^{\prime}}(a)$ e $f_{\mathcal{H}^{\prime}}(b)$ estão em um mesmo componente de $\mathcal{H}^{\prime}$ e o eixo $(a, b)$ em $\mathcal{H}$ admite ao menos uma haste, então as hastes $s_{1}, \ldots, s_{k}$ são arcos de ligação com respeito a $S_{\mathcal{H}^{\prime}}$.

Prova. O primeiro item é uma conseqüência do Lema 3.2 e da definição de arco de ligação. Vamos provar o segundo item.

Se $f_{\mathcal{H}^{\prime}}(a)$ e $f_{\mathcal{H}^{\prime}}(b)$ estão num mesmo componente de $\mathcal{H}^{\prime}$, então $f_{\mathcal{H}}(a)$ e $f_{\mathcal{H}}(b)$ estão num mesmo componente de $\mathcal{H}$ e portanto existe o eixo $(a, b)$ em $\mathcal{H}$. Suponhamos que este eixo admita pelo menos uma haste, digamos $e:=s_{h}$.

Se e não é um arco de ligação, então pelos Lemas 3.2 e 3.5 existe um único componente $\mathcal{W}$ de $\mathcal{H}^{\prime} \operatorname{com} S_{\mathcal{W}} \cap D_{s_{h}} \neq \emptyset$ e $S_{\mathcal{W}} \backslash D_{s_{h}} \neq \emptyset$. Claramente $\mathcal{W}$ é o mesmo componente que contém $f_{\mathcal{H}^{\prime}}(a)$ e $f_{\mathcal{H}^{\prime}}(b)$. Seja $c$ o rótulo de uma folha em $S_{\mathcal{W}} \cap D_{s_{h}}$. Temos, portanto, em $\mathcal{G}$, lca $\left(f_{\mathcal{G}}(a), f_{\mathcal{G}}(b)\right)$ como um descendente estrito de $\operatorname{lca}_{\mathcal{G}}\left(f_{\mathcal{G}}(a), f_{\mathcal{G}}(c)\right)$ e $\operatorname{lca}_{\mathcal{G}}\left(f_{\mathcal{G}}(b), f_{\mathcal{G}}(c)\right)$, enquanto que em $\mathcal{H}$ temos $\operatorname{lca}_{\mathcal{H}}\left(f_{\mathcal{H}}(a), f_{\mathcal{H}}(b)\right)$ como um antepassado estrito de $\operatorname{lca}_{\mathcal{H}}\left(f_{\mathcal{H}}(a), f_{\mathcal{H}}(c)\right)$ ou de $\operatorname{lca}_{\mathcal{H}}\left(f_{\mathcal{H}}(b), f_{\mathcal{H}}(c)\right)$. Como $\mathcal{H}^{\prime}$ é uma floresta de concordância de $\mathcal{G}$ e $\mathcal{H}$, temos uma contradição, pois nenhum componente de $\mathcal{H}^{\prime}$ pode conter três rótulos como $a, b$ e $c$.

Uma conseqüência do lema acima é que a cada iteração $i$ do método básico, ou pelo menos um dos arcos que incidem em $f_{\mathcal{H}_{i}}(a)$ e $f_{\mathcal{H}_{i}}(b)$ são de ligação, ou então 
todas as hastes do eixo $(a, b)$ (quando existentes) são arcos de ligação com relação a $\mathcal{H}^{\prime}$.

Se fosse possível garantir que em cada iteração $i$ do Algoritmo $\mathcal{A}_{\bar{k}}$ pelo menos um arco de ligação de $\mathcal{H}_{i}$ é eliminado, então poderíamos mostrar facilmente que este algoritmo é uma $\max (2, \bar{k})$-aproximação para o problema MAF-2. Isto seria válido pois o algoritmo $\mathcal{A}_{\bar{k}}$ executa a cada iteração no máximo $\max (2, \bar{k})$ operações de corte a partir de $\mathcal{H}_{i}$. Assim, se no início da última iteração $i$ temos que $\mathcal{G}_{i}$ é trivial, então

$$
t-1 \leq i \max (2, \bar{k}) \leq(|\mathcal{F}|-1) \max (2, \bar{k})
$$

o que implica

$$
t \leq|\mathcal{F}| \max (2, \bar{k})-\max (2, \bar{k})+1 \leq|\mathcal{F}| \max (2, \bar{k})
$$

A hipótese de que em cada iteração do Algoritmo $\mathcal{A}_{\bar{k}}$ pelo menos um arco de ligação de $\mathcal{H}_{i}$ com respeito a $\mathcal{H}_{i}^{\prime}$ é eliminado, entretanto, não é válida. No Caso 4 não ocorrem eliminações em $\mathcal{H}_{i}$, e no Caso 3 o Lema 4.5 garante que esta hipótese vale, pois $f_{\mathcal{H}_{i}}(a)$ e $f_{\mathcal{H}_{i}}(b)$ estão necessariamente em componentes de $\mathcal{H}^{\prime}{ }_{i}$ distintos. Nos outros três casos, porém, não podemos garantir a eliminação de ao menos um arco de ligação por iteração. De fato, pode-se verificar que, no exemplo da Figura 4.12, esta hipótese falha tantas vezes quantas são as configurações básicas utilizadas na construção da seção inferior de $\mathcal{U}$. ( $\mathrm{Na}$ Seção 4.5 apresentaremos outro algoritmo que garante a eliminação de um arco de ligação por iterações à custa de um número maior de cortes por iteração.)

Prosseguindo com a análise do Algoritmo $\mathcal{A}_{\bar{k}}$, observamos que o Lema 4.5 garante que, nas iterações em que nenhum arco de ligação é eliminado, existe um conjunto de arcos de ligação "nas proximidades" dos arcos que estão sendo cortados pelo algoritmo. Assim, a idéia é aproveitar estes conjuntos de arcos para compensar a falta de arcos de ligação em algumas iterações. Vamos formalizar esta idéia através de um protocolo de cobrança de taxas. Este protocolo será apresentado em duas partes: um protocolo de créditos e um protocolo de débitos.

\subsubsection{Protocolo de cobrança de taxas}

\subsubsection{Protocolo de créditos}

No que segue, vamos considerar uma execução do Algoritmo $\mathcal{A}_{\bar{k}}$ para uma dada entrada $\mathcal{T}$ e $\mathcal{U}$

Vamos supor que uma taxa unitária deve ser paga cada vez que o Algoritmo $\mathcal{A}_{\bar{k}}$ executa uma operação de corte, enquanto que as operações de encolhimento podem ser executadas sem o pagamento de taxas. Cada taxa deve ser paga com uma unidade 
de crédito, sendo que no início da execução do algoritmo é feita uma provisão inicial destas unidades suficiente para pagar todas as taxas cobradas durante essa execução.

No início da primeira iteração do Algoritmo $\mathcal{A}_{\bar{k}}$, é feito um depósito inicial de unidades de crédito sobre o único componente de $\mathcal{H}_{1}^{1}$ (que é $\mathcal{U}$ ). Ao longo da execução, estas unidades de crédito são guardadas pelos componentes de cada $\mathcal{H}_{i}^{j}$. Quando ocorre uma eliminação em $\mathcal{H}_{i}^{j}$, parte das unidades de crédito guardadas sobre o componente que se particiona é liberada e parte é redistribuída entre os dois componentes resultantes da operação, e depositada sobre eles. Dentre as unidades liberadas, uma parcela pode ser usada para pagar taxas, e o restante expira ao final da iteração (isto é, não pode ser usado para pagar taxas em iterações posteriores).

O objetivo da análise será mostrar que o protocolo acima (a ser detalhado a seguir) permite que, ao término da execução do Algoritmo $\mathcal{A}_{\bar{k}}$, todas as operações de corte tenham sido pagas. Há duas condições que devem ser satisfeitas para que esta afirmação seja válida, e que enunciamos a seguir.

\section{Condição 4.6}

1) O montante do depósito inicial deve ser suficiente para que seja possível efetuar todas as liberações de crédito.

2) A quantidade de crédito liberada cada vez que um arco de ligação é eliminado deve ser suficiente para pagar todas as obrigações daquela iteração.

Na segunda condição acima, entendemos por obrigações tanto as taxas por operações de corte realizadas na mesma iteração em que os créditos são liberados, quanto qualquer montante devido por operações de corte realizadas em iterações anteriores e ainda não pagas. A especificação destes montantes devidos será apresentada mais adiante, na descrição do protocolo de débitos.

De acordo com as regras do protocolo acima, para cada arco de ligação eliminado deve ocorrer uma liberação de crédito. Se, para cada $\bar{k} \geq 1$, a quantidade de crédito liberada quando um arco de ligação é eliminado é dada por uma constante $\alpha_{\bar{k}} \geq 1$, e $\mathcal{F}$ é a floresta de concordância ótima que tomamos como referência na Seção 4.4.2, então podemos considerar o montante do depósito inicial como sendo $\alpha_{\bar{k}}(|\mathcal{F}|-1)$, lembrando que o número de arcos de ligação eliminados em uma execução de $\mathcal{A}_{\bar{k}}$ é $|\mathcal{F}|-1$ pelo Lema 4.2. Com isso, satisfazemos o primeiro item da Condição 4.6.

Vamos em seguida verificar que, durante a execução de $\mathcal{A}_{\bar{k}}$, as unidades de crédito se conservam.

Para cada $i \leq 1$ e $j=1, \ldots, \bar{\jmath}_{i}$, seja $\mathcal{W}$ o componente de $\mathcal{H}_{i}^{j}$ que se particiona após a operação $h_{i}^{j}$, e seja $e$ o arco de $\mathcal{H}_{i}^{j}$ eliminado nesta operação. De acordo com 
a definição de eliminação, a operação $h_{i}^{j}$ acarreta a troca de $\mathcal{W}$ pelos componentes $\mathcal{W} \mid D_{e}$ e $\mathcal{W} \mid\left(S_{\mathcal{W}} \backslash D_{e}\right)$ em $\mathcal{H}_{i}^{j}$

Sejam:

1) $m^{\mathcal{W}}:=$ número de componentes $\mathcal{W}^{\prime}$ de $\mathcal{H}_{i}^{\prime j}$ tais que $S_{\mathcal{W}^{\prime}} \subseteq S_{\mathcal{W}}$

2) $m^{\backslash}:=$ número de componentes $\mathcal{W}^{\prime}$ de $\mathcal{H}_{i}^{\prime j+1}$ tais que $S_{\mathcal{W}^{\prime}} \subseteq S_{\mathcal{W}} \backslash D_{e}$, e

3) $m^{\cap}:=$ número de componentes $\mathcal{W}^{\prime}$ de $\mathcal{H}_{i}^{\prime j+1}$ tais que $S_{\mathcal{W}^{\prime}} \subseteq D_{e}$.

Considere que manipulamos o crédito sobre $\mathcal{W}$ segundo as regras abaixo:

1) Se $e$ é um arco de ligação com relação a $\mathcal{H}_{i}^{\prime j}$, então das $\alpha_{\bar{k}}\left(m^{\mathcal{W}}-1\right)$ unidades de crédito guardadas por $\mathcal{W}, \alpha_{\bar{k}}$ unidades são liberadas, $\alpha_{\bar{k}}\left(m^{\cap}-1\right)$ unidades são depositadas sobre $\mathcal{W} \mid D_{e}$ e $\alpha_{\bar{k}}(m \backslash-1)$ unidades são depositadas sobre $\mathcal{W} \mid\left(S_{\mathcal{W}} \backslash\right.$ $\left.D_{e}\right)$.

Como neste caso vale $m^{\mathcal{W}}=m^{n}+m$, então o total de créditos se conserva durante a operação.

2) Se $e$ não é um arco de ligação com relação a $\mathcal{H}^{\prime j}{ }_{i}$, então os $\alpha_{\bar{k}}\left(m^{\mathcal{W}}-1\right)$ créditos associados a $\mathcal{W}$ são divididos em $\alpha_{\bar{k}}\left(m^{n}-1\right)$ créditos para $\mathcal{W} \mid D_{e}$ e $\alpha_{\bar{k}}(m \backslash-1)$ créditos para $\mathcal{W} \mid\left(S_{\mathcal{W}} \backslash D_{e}\right)$, e nenhum crédito é liberado.

Como neste caso vale $m^{\mathcal{W}}=m^{\cap}+m^{\backslash}-1$, então o total de créditos também se conserva.

Se depositamos inicialmente $\alpha_{\bar{k}}(|\mathcal{F}|-1)$ créditos sobre $\mathcal{U}$, então a discussão acima demonstra o seguinte lema:

Lema 4.7 Seja $i$ uma iteração do Algoritmo $\mathcal{A}_{\bar{k}}$, e $j$ tal que $1 \leq j \leq \bar{\jmath}_{i}$. Então cada componente $\mathcal{W}$ de $\mathcal{H}_{i}^{j}$ guarda $\alpha_{\bar{k}}\left(m^{\mathcal{W}}-1\right)$ créditos, sendo $m^{\mathcal{W}} \bar{o}$ número de componentes $\mathcal{W}^{\prime}$ de $\mathcal{H}^{\prime j}$ tais que $S_{\mathcal{W}^{\prime}} \subseteq S_{\mathcal{W}}$.

Observe que inicialmente temos $m^{\mathcal{H}_{1}^{1}}=|\mathcal{F}|$ e $m^{n}=m \backslash=0$.

A Figura 4.16 traz alguns exemplos de cortes a partir de uma árvore filogenética, com a correspondente redistribuição de crédito. No exemplo da figura, o depósito inicial é de $4 \alpha_{\bar{k}}$ unidades de crédito e $m^{\mathcal{W}}=5$. Os arcos em negrito indicam os componentes $\mathcal{W}^{\prime}$ de $\mathcal{H}_{i}^{\prime j}$ tais que $S_{\mathcal{W}^{\prime}} \subseteq S_{\mathcal{W}}$. A primeira eliminação em $\mathcal{W}$ é feita sobre um arco de ligação, produzindo os componentes $\mathcal{W}^{n}$ com $\alpha_{\bar{k}}$ unidades de crédito e $m^{n}=2$, e $\mathcal{W} \backslash$ com $2 \alpha_{\bar{k}}$ unidades de crédito e $m \backslash=3$. A segunda eliminação é feita sobre um arco que não é de ligação, dividindo $\mathcal{W} \backslash$ em dois componentes: $\mathcal{W}^{\backslash n}$ com $\alpha_{\bar{k}}$ unidades de crédito e $m \backslash \cap=2$, e $\mathcal{W} \backslash \backslash$ com $\alpha_{\bar{k}}$ unidades de crédito e $m \backslash \backslash=2$. Na 

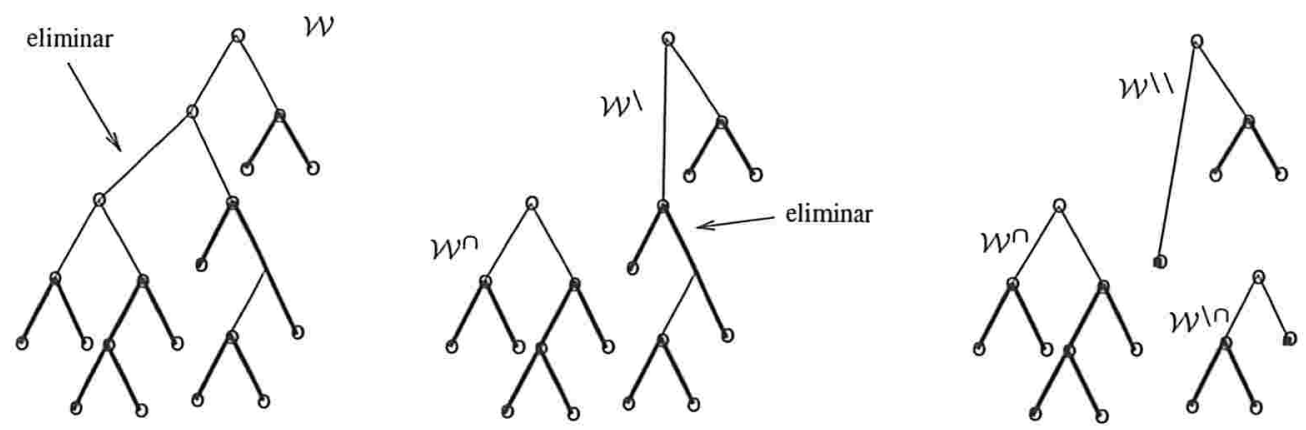

Figura 4.16: Um exemplo de redistribuição de crédito com duas eliminações.

primeira eliminação, são liberadas $\alpha_{\bar{k}}$ unidades de crédito, enquanto que na segunda eliminação, não ocorre liberação de créditos.

Observe que, neste protocolo, o montante de depósito inicial, de $\alpha_{\bar{k}}(|\mathcal{F}|-1)$ unidades de crédito, está relacionado ao tamanho da solução ótima $\mathcal{F}$ para o problema MAF-2 com instância $\mathcal{T}, \mathcal{U}$, enquanto que a soma de todas as taxas unitárias relaciona-se com o tamanho da solução devolvido pelo Algoritmo $\mathcal{A}_{\bar{k}}$. O objetivo da análise daqui por diante será mostrar que o segundo item da Condição 4.6 pode ser satisfeito para um determinado valor de $\alpha_{\bar{k}}$.

Esta técnica de análise elabora o argumento esboçado em Hein et al. [20, 19] para justificar a afirmação - que não é correta, conforme mostramos na Seção 4.4.1 — de que o Algoritmo $\mathcal{A}_{1}$ é um algoritmo de 3-aproximação. Em nossa análise, acrescentamos ao argumento em questão um controle mais rigoroso sobre o comportamento das unidades de crédito e débito, de modo a eliminar os pontos falhos da justificativa em $[20,19]$.

\subsubsection{Protocolo de débitos}

De acordo com o protocolo de créditos, as taxas das operações de corte de cada iteração devem ser pagas com unidades de crédito liberadas na mesma iteração, pois o crédito liberado expira de uma iteração para a iteração seguinte. A liberação de crédito ocorre apenas quando um arco de ligação é eliminado. Entretanto, vimos na seção anterior que podem ocorrer iterações em que não há eliminação de arcos de ligação. Portanto, nas iterações em que são executados cortes mas nenhum arco de ligação é eliminado, não há unidades de crédito com que pagar as taxas das operações de corte realizadas nestas iterações.

O protocolo de cobrança de taxas resolve este problema utilizando unidades de débito. Se em uma iteração ocorrem cortes mas nenhum arco de ligação é eliminado, então são emitidas unidades de débito em quantidade equivalente às taxas a serem 


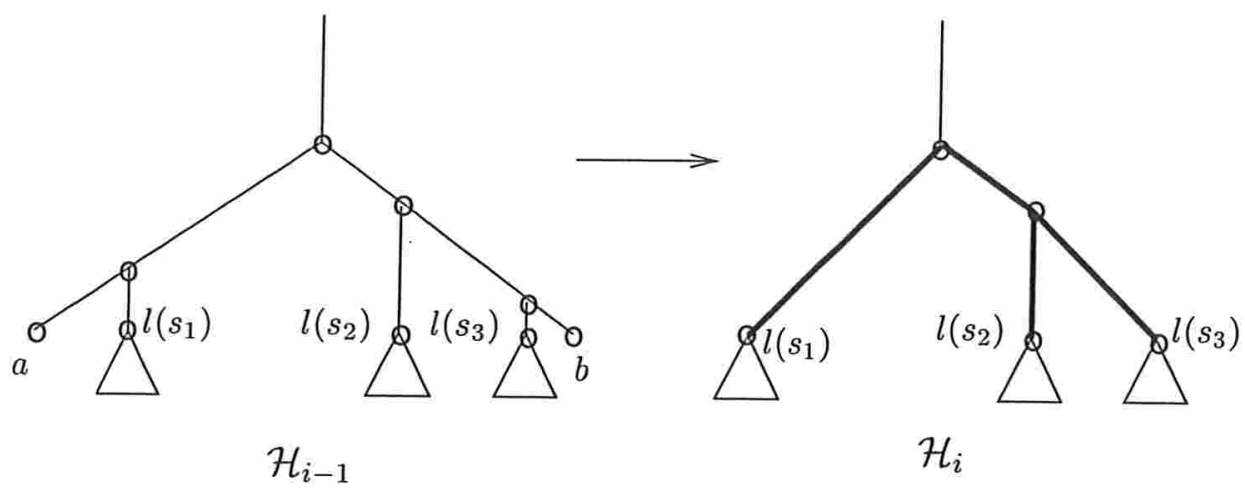

Figura 4.17: Um exemplo de resíduo de hastes.

pagas, sendo que cada taxa unitária é paga com uma destas unidades. As unidades de débito, ao contrário das unidades de crédito, não expiram, e devem estar todas quitadas com unidades de crédito ao encerramento da execução.

Vimos que unidades de crédito são guardadas durante a execução do Algoritmo $\mathcal{A}_{\bar{k}}$ pelos componentes das restrições $\mathcal{H}_{i}^{j}$. Com as unidades de débito ocorre algo semelhante, pois elas também permanecem ligadas a alguns elementos estruturais de $\mathcal{H}_{i}^{j}$ até o momento em que são quitadas. Para especificar estes elementos, precisamos introduzir algumas definições.

Seja $\mathcal{W}$ uma árvore filogenética, e $B$ um conjunto de nós de $\mathcal{W}$ com pelo menos dois elementos. Denotamos por $\left.\mathbf{U P}_{\mathcal{W}}(B)\right)$ o conjunto dos arcos cujas extremidades superiores são os lcas em $\mathcal{W}$ de pelo menos dois nós de $B$.

Se para algum $i \geq 2$ a iteração $i-1$ é de Caso 2, então denominamos resíduo das hastes $s_{1}, \ldots, s_{k}$ o conjunto de $\operatorname{arcos} \operatorname{UP}_{\mathcal{H}_{i}}\left(\left\{l\left(s_{h}\right): 1 \leq h \leq k\right\}\right)$. A Figura 4.17 mostra um exemplo de resíduo, com arcos realçados em negrito.

Iterações em que ocorrem cortes mas não há eliminação de arcos de ligação a partir de $\mathcal{H}_{i-1}$ podem ser de dois tipos:

1) a iteração $i-1$ é de Caso 1 em que $s_{h}$ não é um arco de ligação em $\mathcal{H}_{i-1}^{h}$ para $h=1, \ldots, \bar{k}$;

2) a iteração $i-1$ é de Caso 2 em que $f_{\mathcal{H}_{i-1}}(a)$ e $f_{\mathcal{H}_{i-1}}(b)$ estão num mesmo componente de $\mathcal{H}_{i-1}^{\prime}$ e este componente tem pelo menos mais uma folha.

Na situação (1), temos pelo Lema 4.5 que um dos arcos incidindo em $f_{\mathcal{H}_{i-1}}(a)$ e $f_{\mathcal{H}_{i-1}}(b)$ é de ligação com relação a $\mathcal{H}_{i}$. Na situação (2), o Lema 4.5 garante que as hastes $s_{1}, \ldots, s_{k}$ são arcos de ligação com relação a $\mathcal{H}_{i-1}$, e portanto os arcos do resíduo das hastes também são de ligação. Assim, em ambos os casos, existem 
Capítulo 4. Algoritmos de aproximação para árvores com grau no máximo 268

conjuntos de arcos de ligação que serão eliminados em alguma iteração futura, e a cujo crédito liberado podemos recorrer para pagar taxas em iterações em que não há eliminação de arcos de ligação.

Portanto, podemos resolver o problema das iterações em que não há liberação de crédito tomando as unidades de débito emitidas nestas iterações e associando-as com os conjuntos de arcos de ligação que identificamos nos itens (1) e (2) acima. No item (1), associamos a cobrança a um único arco, e no item (2), associamos a cobrança ao resíduo das hastes. Deste modo podemos garantir que, se o crédito liberado no futuro por estes conjuntos é suficiente (ou seja, se o segundo item da Condição 4.6 é válido), então o débito associado a eles pode ser quitado durante a execução do algoritmo.

Finalizamos esta seção ressaltando um detalhe importante: o fato de associarmos unidades de débito a conjuntos de arcos. Esta idéia é fundamental para o sucesso da técnica nos casos em que associamos débito com resíduos, pois ela permite que o débito associado seja pago em quotas de igual tamanho à medida que os arcos do resíduo vão sendo eliminados.

A seção seguinte completa a descrição do protocolo de débitos, especificando o que ocorre com as unidades de débito à medida que os conjuntos de arcos aos quais elas se associam são modificados pelas operações do Algoritmo $\mathcal{A}_{\bar{k}}$.

\subsubsection{Barreiras}

Na seção anterior mencionamos resíduos de hastes como sendo os conjuntos de arcos de ligação ao qual associamos unidades de débito quando ocorre a situação (2). Na verdade, vamos associar débitos a um tipo mais geral de conjunto de arcos de ligação, que introduzimos nesta seção.

Resíduos formam-se quando temos uma iteração $i-1$ de Caso 2 em que $f_{\mathcal{H}_{i-1}}(a)$ e $f_{\mathcal{H}_{i-1}}(b)$ estão num mesmo componente de $\mathcal{H}_{i-1}^{\prime}$ e este componente tem pelo menos mais uma folha além de $f_{\mathcal{H}_{i-1}}(a)$ e $f_{\mathcal{H}_{i-1}}(b)$. Neste caso, como o algoritmo não elimina nenhum arco de ligação, não são liberadas unidades de crédito com que pagar as taxas das operações de corte.

O Lema 4.5 garante que neste caso as hastes do eixo $(a, b)$ são arcos de ligação. Portanto, no início da iteração seguinte o resíduo destas hastes também é composto por arcos de ligação, e podemos associar as unidades de débito emitidas a este resíduo.

Este é o protocolo que delineamos na Seção 4.4.3.2. Entretanto, precisamos considerar também um outro tipo de conjunto de arcos de ligação para associação de débitos, conforme explicamos abaixo.

Seria desejável que associações de débito a resíduos fossem mantidas até que todas as unidades de débito associadas tivessem sido quitadas. Entretanto, estes resíduos são particionados ao longo da execução do Algoritmo $\mathcal{A}_{\bar{k}}$, à medida que seus arcos 
são eliminados.

Nestes casos, as unidades de débito ainda não quitadas precisam ser novamente associadas a algum outro conjunto de arcos de ligação. Uma escolha lógica é, a cada eliminação de um arco em um resíduo com débito associado, reassociar o débito não quitado aos dois conjuntos de arcos em que este resíduo se particiona.

Seja $\mathcal{H}_{i}^{j}$ uma restrição com $(i, j) \neq(1,1)$, e seja $e$ o arco cuja eliminação produziu $\mathcal{H}_{i}^{j}$. Um conjunto de nós $B$ em $\mathcal{H}_{i}^{j}$ com pelo menos dois nós é uma barreira se:

- $B$ é uma barreira primária. Ou seja, $j=1$; a iteração $i-1$ é de Caso 2 , com $f_{\mathcal{H}_{i-1}}(a)$ e $f_{\mathcal{H}_{i-1}}(b)$ pertencendo a um componente de $\mathcal{H}^{\prime}{ }_{i-1}$ que tem pelo menos mais uma folha; $B=\left\{l\left(s_{h}\right): 1 \leq h \leq k\right\}$; ou

- $B$ admite uma barreira antecessora $B^{\prime}$. Isto é, $j \geq 2$ e existe uma barreira $B^{\prime}$ em $\mathcal{H}_{i}^{j-1}$ tal que vale uma das condições abaixo:

$$
\begin{aligned}
& \text { - }\left(e \in \mathrm{UP}_{\mathcal{H}_{i}^{j-1}}\left(B^{\prime}\right)\right) ; B=B^{\prime} \cap D_{e} \text { ou } B=B^{\prime} \backslash D_{e} ; \\
& \text { - }\left(e \notin \mathrm{UP}_{\mathcal{H}_{i}^{j-1}}\left(B^{\prime}\right)\right) \text { e } B=B^{\prime} .
\end{aligned}
$$

Neste caso, $B$ é uma barreira sucessora de $B^{\prime}$.

A Figura 4.18 mostra os casos possíveis de barreiras primárias. Na primeira coluna mostramos $\mathcal{H}_{i-1}$ para cada um dos cinco casos listados, e na segunda coluna temos $\mathcal{H}_{i}$ com os resíduos em negrito. $\mathrm{O}$ inteiro $k^{\prime}$ denota o número de hastes que estão do mesmo lado que o rótulo $a$ no eixo $(a, b)$ em $\mathcal{H}_{i-1}$.

A Figura 4.19 lista todos os casos possíveis de barreiras com antecessoras. Sejam $e, e_{1}$ e $e_{2}$ os arcos mostrados na Figura 4.1 para a eliminação feita na operação que resultou na restrição $\mathcal{H}_{i}^{j}$. Na coluna esquerda, estão marcadas as barreiras antecessoras $B^{\prime}$ em $\mathcal{H}_{i}^{j-1}$, e na coluna direita estão assinaladas as barreiras sucessoras $B$ de $B^{\prime}$ em $\mathcal{H}_{i}^{j}$. Alguns casos da definição estão desmembrados em subcasos na figura. $\mathrm{O}$ arco $e$ está em negrito.

Damos a seguir algumas propriedades das barreiras.

Lema 4.8 Seja $\mathcal{H}_{i}^{j}$ tal que $(i, j) \neq(1,1)$, e seja $B$ uma barreira em $\mathcal{H}_{i}^{j}$. Então todos os arcos em $U P_{\mathcal{H}_{i}^{j}}(B)$ são arcos de ligação.

Prova. Este lema é uma conseqüência imediata do Lema 4.5 se $B$ é uma barreira primária, e pode ser provada por indução se $B$ é uma barreira sucessora.

O Lema 4.8 tem um papel importante na análise de desempenho do algoritmo. 
Capítulo 4. Algoritmos de aproximação para árvores com grau no máximo 270

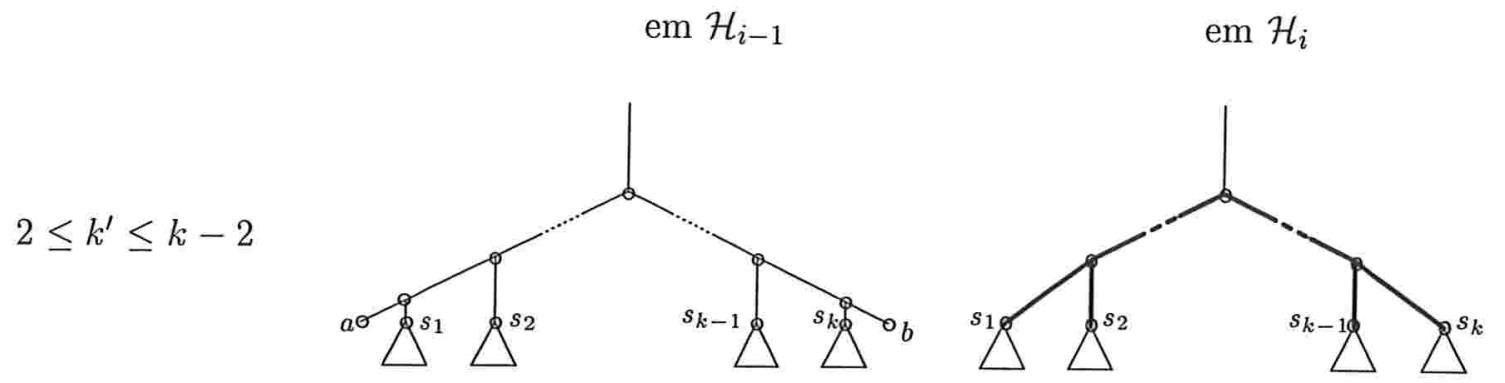

$$
\begin{gathered}
k^{\prime}=1 \text { ou } \\
k^{\prime}=k-1 \\
k \geq 3
\end{gathered}
$$
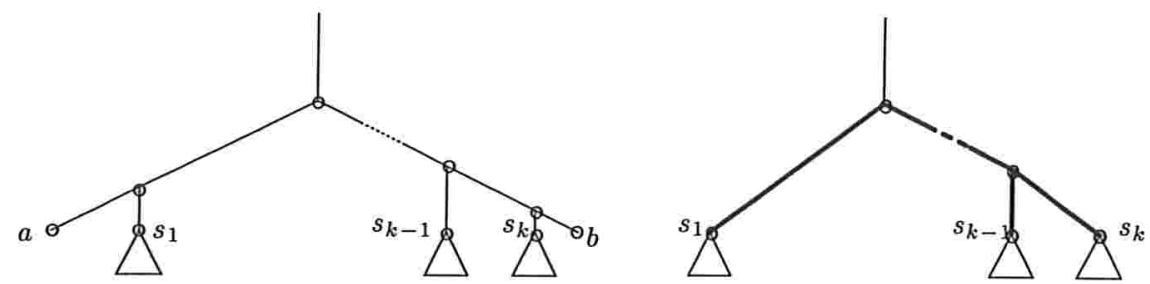

$$
\begin{gathered}
k^{\prime}=1 \text { ou } \\
k^{\prime}=k-1 ; \\
k=2
\end{gathered}
$$
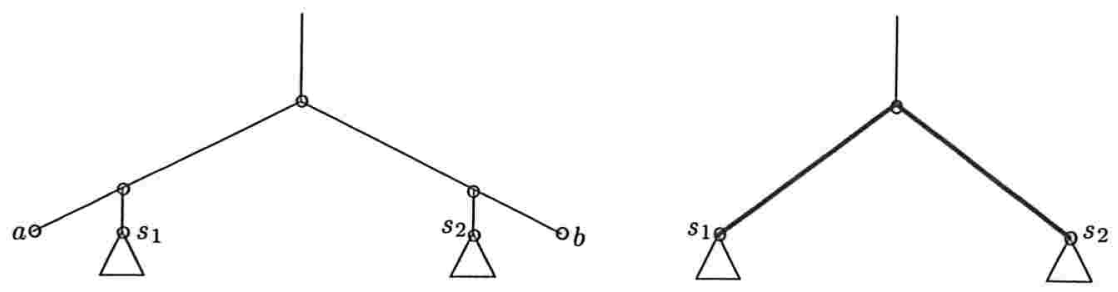

$$
\begin{gathered}
k^{\prime}=0 \text { ou } \\
k^{\prime}=k ; \\
k \geq 3
\end{gathered}
$$
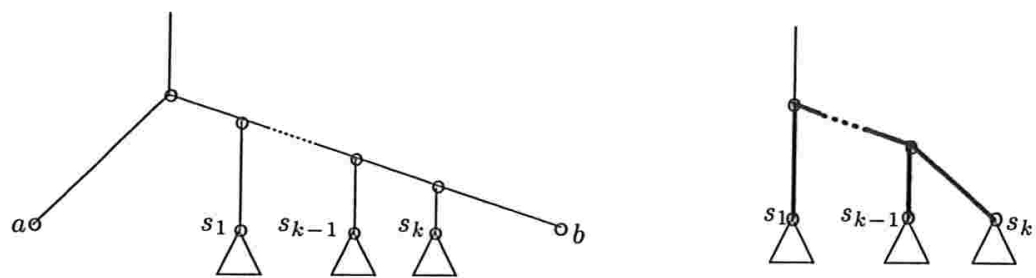

$$
\begin{gathered}
k^{\prime}=0 \text { ou } \\
k^{\prime}=k \\
k=2
\end{gathered}
$$
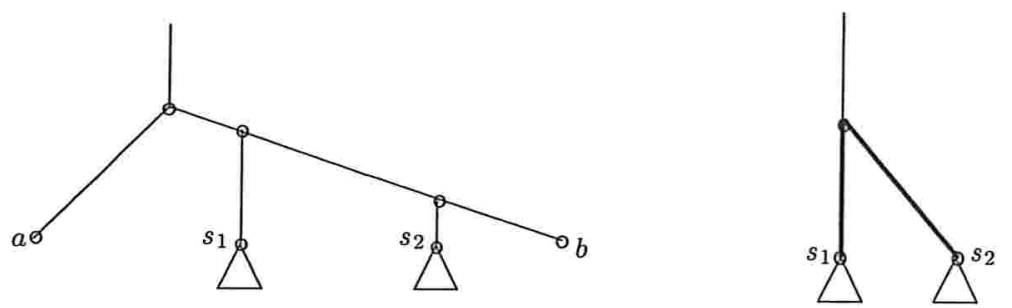

Figura 4.18: Barreiras primárias. 
Capítulo 4. Algoritmos de aproximação para árvores com grau no máximo 271

$e \in \mathrm{UP}_{\mathcal{H}_{i}^{j-1}}\left(B^{\prime}\right)$

$\exists e_{2}$ e $e_{2} \in \mathrm{UP}_{\mathcal{H}_{i}^{j-1}} B^{\prime}$

em $\mathcal{H}_{i}^{j-1}$

em $\mathcal{H}_{i}^{j}$
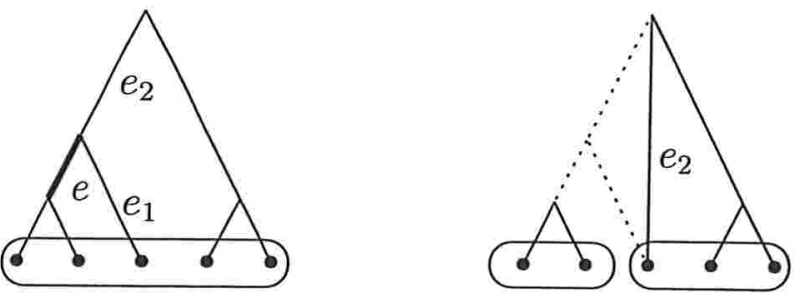

$e \in \mathrm{UP}_{\mathcal{H}_{i}^{j-1}}\left(B^{\prime}\right)$

$\nexists e_{2}$ ou $\left(\exists e_{2}\right.$ e $\left.e_{2} \notin \mathrm{UP}_{\mathcal{H}_{i}^{j-1}}\left(B^{\prime}\right)\right)$
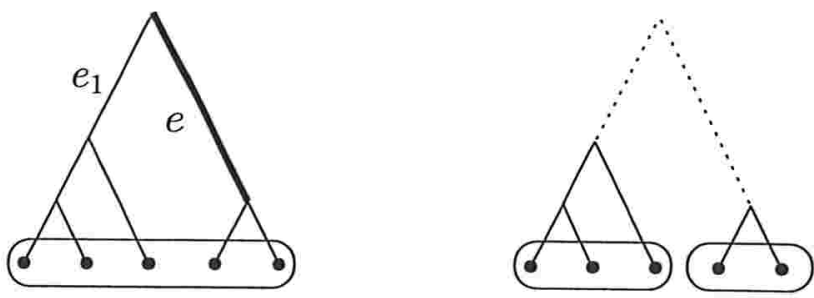

$e \notin \mathrm{UP}_{\mathcal{H}_{i}^{j-1}}\left(B^{\prime}\right)$

$\nexists e_{2}$ ou $\left(\exists e_{2}\right.$ e $\left.e_{2} \notin U \mathrm{UP}_{\mathcal{H}_{i}^{j-1}}\left(B^{\prime}\right)\right)$
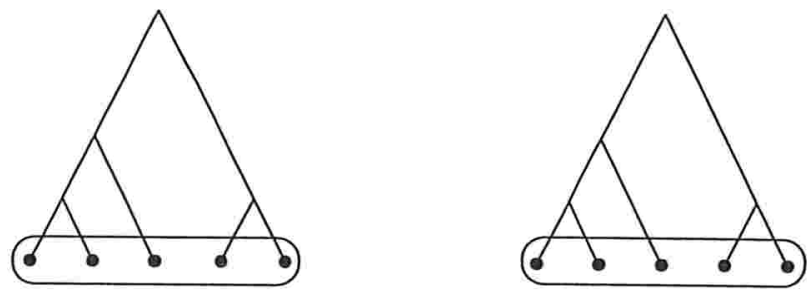

$e \notin \mathrm{UP}_{\mathcal{H}_{i}^{j-1}}\left(B^{\prime}\right)$

$\exists e_{2}$ e $e_{2} \in \mathrm{UP}_{\mathcal{H}_{i}^{j-1}}\left(B^{\prime}\right)$
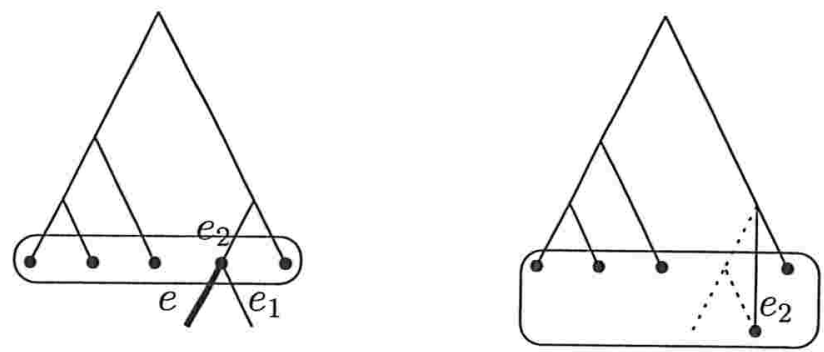

Figura 4.19: Barreiras com antecessoras. 

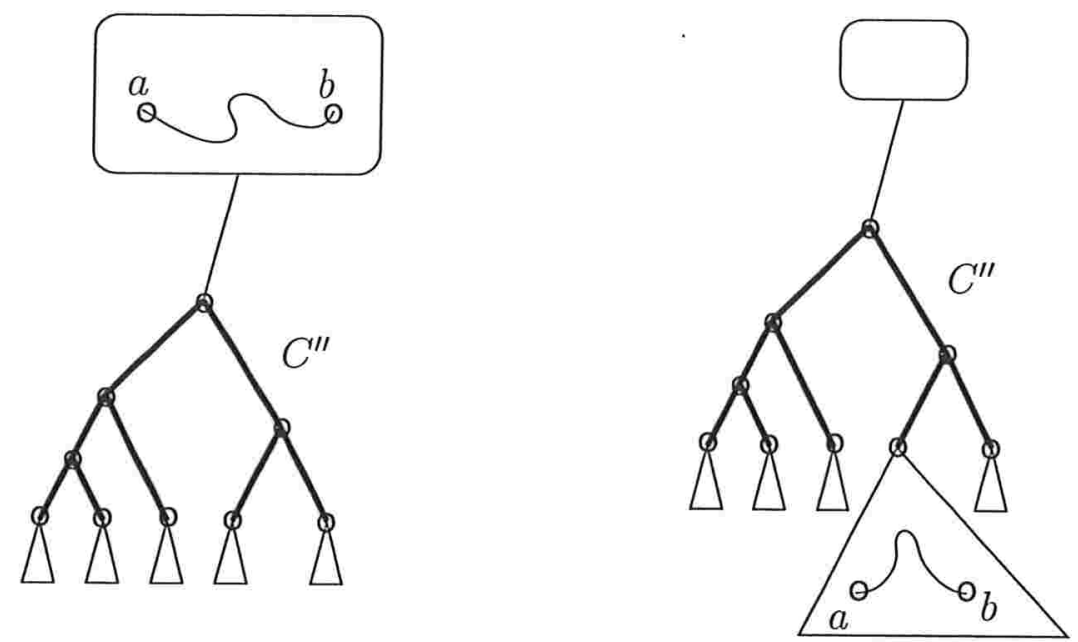

Figura 4.20: Restrição $\mathcal{H}_{i-1}$ e barreira antecessora $C^{\prime \prime}$ na prova do Lema 4.9.

Lema 4.9 Seja $\mathcal{H}_{i}^{j}$ tal que $(i, j) \neq(1,1)$, e considere duas barreiras distintas $B$ e $C$ em $\mathcal{H}_{i}^{j}$. Então $U P_{\mathcal{H}_{i}^{j}}(B)$ e UP $\mathcal{H}_{i}^{j}(C)$ são disjuntos nos arcos.

Prova. A prova é feita por indução no número de operações desde que a barreira primária mais recente, dentre as barreiras primárias que deram origem a $B$ e $C$, foi criada. A base da indução é quando este número de operações é zero, ou seja, quando $B$ é primária ou $C$ é primária.

Suponhamos sem perda de generalidade que $B$ seja primária. Então:

- A restrição $\mathcal{H}_{i-1}$ satisfaz as condições da definição de barreira primária, e portanto foram feitas duas operações a partir de $\mathcal{H}_{i-1}$ na iteração $i-1$ (isto é, $\left.\bar{\jmath}_{i-1}=2\right)$.

- $C$ admite uma barreira antecessora em $\mathcal{H}_{i-1}^{2}$, que vamos chamar de $C^{\prime}$, e $C^{\prime}$ admite uma barreira antecessora em $\mathcal{H}_{i-1}^{1}=\mathcal{H}_{i-1}$, que chamamos de $C^{\prime \prime}$.

A Figura 4.20 esquematiza $C^{\prime \prime}$ em $\mathcal{H}_{i-1}$, mostrando os dois casos possíveis em que $B$ é uma barreira primária em $\mathcal{H}_{i}$. O conjunto $\mathrm{UP}_{\mathcal{H}_{i-1}}\left(C^{\prime \prime}\right)$ está indicado em negrito.

Considere o eixo $(a, b)$ em $\mathcal{H}_{i-1}$, e suponha que o caminho entre lca $(a, b)$ e $r_{\mathcal{H}_{i-1}}$ não passa por lca $\left(C^{\prime \prime}\right)$ (lado esquerdo da Figura 4.20). Pela definição de barreira primária, todos os arcos deste eixo estão em um mesmo componente de $\mathcal{H}_{i-1}^{\prime}$, e portanto nenhum dos arcos de $\mathrm{UP}_{\mathcal{H}_{i-1}}\left(C^{\prime \prime}\right)$ (que são de ligação, de acordo com o Lema 4.8) é usado pelo eixo. Como o arco $s\left(\operatorname{lca}\left(C^{\prime \prime}\right)\right)$ é de ligação, então $\mathrm{UP}_{\mathcal{H}_{i}^{j}}(B)$ e $\mathrm{UP}_{\mathcal{H}_{i}^{j}}(C)$ são disjuntos nos arcos. A prova é análoga para o caso em que o caminho entre lca $(a, b)$ e $r_{\mathcal{H}_{i-1}}$ passa por lca $\left(C^{\prime \prime}\right)$ (lado direito da Figura 4.20). 
Capítulo 4. Algoritmos de aproximação para árvores com grau no máximo 273

O passo da indução é quando $B$ e $C$ admitem barreiras antecessoras. Podemos facilmente verificar que a tese segue da hipótese de indução para todos os casos listados na Figura 4.19.

O Lema 4.9 é fundamental para a análise. Ele garante que barreiras primárias não se sobrepõem a barreiras sucessoras pré-existentes e também que não ocorre fusão entre barreiras diferentes à medida que o algoritmo segue eliminando arcos. Os dois fatos juntos certificam que nenhuma barreira pode ter mais unidades de débito associadas do que o número máximo de operações de corte por iteração de Caso 2.

Em vista disso, o débito associado a cada barreira primária é dividido em quotas de mesmo montante. Cada quota é quitada quando cortamos um arco de ligação na barreira, e as quotas restantes (se houver alguma) são associadas com as barreiras sucessoras.

Toda barreira primária de cardinalidade $k$ gera $k-2$ barreiras sucessoras, e portanto permite o pagamento de $k-1$ quotas. Como a cardinalidade mínima de uma barreira primária é $\bar{k}+1$ e o débito máximo associado a uma barreira primária é de duas unidades de débito, as quotas devem ser de $2 / \bar{k}$ unidades de débito.

\subsubsection{Limitantes superiores}

Nesta seção vamos verificar a validade do segundo item da Condição 4.6.

Temos vários casos a considerar na nossa análise:

1) iterações Caso 1 em que nenhum arco de ligação é eliminado;

2) iterações Caso 2 em que nenhum arco de ligação é eliminado;

3) iterações Caso 3 em que nenhum arco de ligação é eliminado;

4) iterações Caso 4 em que nenhum arco de ligação é eliminado;

5) iterações Caso 5 em que nenhum arco de ligação é eliminado;

6) iterações Caso 1 em que eliminamos pelo menos um arco de ligação.

7) iterações Caso 2 em que eliminamos pelo menos um arco de ligação.

8) iterações Caso 3 em que eliminamos pelo menos um arco de ligação.

9) iterações Caso 4 em que eliminamos pelo menos um arco de ligação.

10) iterações Caso 5 em que eliminamos pelo menos um arco de ligação. 
No primeiro caso, conforme o protocolo de débitos, no máximo $\bar{k}$ unidades de débito são emitidas e associadas com um dos arcos incidentes em $f_{\mathcal{H}_{i}}(a)$ e $f_{\mathcal{H}_{i}}(b)$; sabemos que pelo menos um destes arcos é de ligação. Este arco só será eliminado posteriormente em uma iteração Caso 5. Sem perda de generalidade, podemos supor que o arco que incide em $f_{\mathcal{H}_{i}}(b)$ é de ligação, pois podemos permutar os rótulos $a$ e $b$ em $\mathcal{H}^{\prime}{ }_{i}$ em uma iteração Caso 5.

No segundo caso temos a formação de uma barreira primária, conforme discutido na Seção 4.4.3.3. A esta barreira inicialmente associamos duas unidades de débito, que são divididas em quotas de $2 / \bar{k}$ unidades, sendo que cada quota deve ser quitada quando um arco da barreira é eliminado.

O terceiro caso jamais ocorre por causa do Lema 4.5, enquanto que no quarto e no penúltimo casos não ocorre nenhuma eliminação em $\mathcal{H}_{i}$.

No quinto caso não ocorrem cortes, e portanto não são emitidas novas unidades de débito.

Observe que nos cinco primeiros casos não ocorrem liberação de unidades de crédito ou quitação, já que nestes casos não estamos eliminando arcos de ligação.

No sexto caso, de acordo com a transação aplicada no Caso 1 (ver a Tabela 4.1 para a definição das transações, e a Figura 4.4 para a definição dos casos do método básico), devem ser pagos no máximo $\bar{k}$ cortes, correspondentes às hastes eliminadas pela transação. Adicionalmente, cada arco de ligação eliminado poder pertencer a uma barreira, e para cada arco de ligação pertencente a uma barreira que é eliminado deve ser quitada uma quota de $2 / \bar{k}$ unidades de débito. Se $j$ arcos de ligação são eliminados, então devemos ter $j \alpha_{\bar{k}} \geq \bar{k}+j(2 / \bar{k})$ para satisfazer o segundo item da Condição 4.6 neste caso.

No sétimo e no oitavo casos, devem ser pagos por iteração exatamente 2 cortes, mais uma quota de $2 / \bar{k}$ unidades de débito para cada arco de ligação eliminado que pertença a uma barreira. Se $j$ arcos de ligação são eliminados, então para satisfazer o segundo item da Condição 4.6, devemos ter $j \alpha_{\bar{k}} \geq 2+j(2 / \bar{k})$.

Finalmente, no último caso, podemos ter no máximo $\bar{k}$ unidades de débito associadas ao arco incidente em $f_{\mathcal{H}_{i}}(b)$ (que é eliminado sem nenhum custo), mais uma quota de $2 / \bar{k}$ unidades de débito a ser paga se este arco pertencer a uma barreira. Portanto, devemos ter $\alpha_{\bar{k}} \geq \bar{k}+2 / \bar{k}$.

Portanto, para satisfazer a demanda por unidades de crédito em todas essas situações, é suficiente fazer $\alpha_{\bar{k}}:=\max (2, \bar{k})+2 / \bar{k}$. Com isso, mostramos que o segundo item da Condição 4.6 é válido, e temos que o montante do depósito inicial (que é de $\alpha_{\bar{k}}(|\mathcal{F}|-1)$ unidades de crédito) é suficiente para pagar todas as taxas durante a execução de $\mathcal{A}_{\bar{k}}$; ou seja, $t-1 \leq \alpha_{\bar{k}}(|\mathcal{F}|-1)$.

Com isso, provamos o seguinte resultado: 
Capítulo 4. Algoritmos de aproximação para árvores com grau no máximo 275

Teorema 4.10 Para cada inteiro $\bar{k} \geq 1$, o Algoritmo $\mathcal{A}_{\bar{k}}$ é uma $\max (2, \bar{k})+2 / \bar{k}$ aproximação para o problema $M A F-\mathscr{Q}$.

A razão de aproximação $\max (2, \bar{k})+2 / \bar{k}$ é mínima para $\bar{k}=2$, sendo nesse caso igual a 3. Com isso, temos que o Algoritmo $\mathcal{A}_{2}$ para o problema MAF-2 possui razão de aproximação 3. Já o algoritmo proposto por Hein et al. [20,19], que é $\mathcal{A}_{1}$, possui razão de aproximação 4.

Da Seção 4.4.1 temos que estas razões, 4 e 3 , são justas para $\mathcal{A}_{1}$ e para $\mathcal{A}_{2}$, respectivamente. Para $\bar{k} \geq 3$, sabemos que a menor razão de aproximação do Algoritmo $\mathcal{A}_{\bar{k}}$ está entre $2+2 / \bar{k}$ e $\max (2, \bar{k})+2 / \bar{k}$.

Com isso, provamos os seguintes resultados:

Teorema 4.11 $\mathrm{O}$ Algoritmo $\mathcal{A}_{2}$ é uma 3-aproximação para o problema MAF-2. Ademais, a razâo 3 é justa para este algoritmo.

Teorema 4.12 $O$ Algoritmo $\mathcal{A}_{1}$ é uma 4-aproximação para o problema MAF-2. Ademais, a razão \& é justa para este algoritmo.

Teorema 4.13 O problema MAF-2 é 3-aproximável.

\section{5 $O$ algoritmo de aproximação $\mathcal{B}$}

Vimos na Seção 4.4.2 que um algoritmo obtido a partir do método básico cujas transações sejam capazes de eliminar um arco de ligação com relação à restrição completa $\mathcal{H}^{\prime j}$ de $\mathcal{H}_{i}^{j}$ para $i \geq 1$ e $j=1, \ldots, \bar{j}_{i}$ possui razão de aproximação igual ao número máximo de arcos cortados em $\mathcal{H}_{i}$ por transação. Vimos também que as transações do Algoritmo $\mathcal{A}_{\bar{k}}$ não satisfazem esta propriedade.

Exibimos a seguir um conjunto de transações para o método básico que cortam no máximo três arcos em $\mathcal{H}_{i}^{j}$ e eliminam pelo menos um arco de ligação por iteração. A Tabela 4.2 mostra estas transações. Chamamos de Algoritmo $\mathcal{B}$ o novo algoritmo cujas transações estão especificadas nessa tabela.

Não é difícil verificar, por indução nas seqüências de operações $\left(g_{i}^{j}\right)_{i, j}$ e $\left(h_{i}^{j}\right)_{i, j}$ executadas por $\mathcal{B}$ a partir de $\mathcal{T}$ e $\mathcal{U}$, que os três itens do Lema 4.3 valem para $\mathrm{o}$ Algoritmo $\mathcal{B}$, e que este algoritmo devolve o tamanho de uma floresta de concordância de $\mathcal{T}$ e $\mathcal{U}$.

O Algoritmo $\mathcal{B}$ pode ser implementado em tempo quadrático no número $n$ de folhas nas árvores $\mathcal{T}$ e $\mathcal{U}$ dadas como entrada. $\mathrm{O}$ argumento é o mesmo que empregamos para o Algoritmo $\mathcal{A}_{\bar{k}}$. 


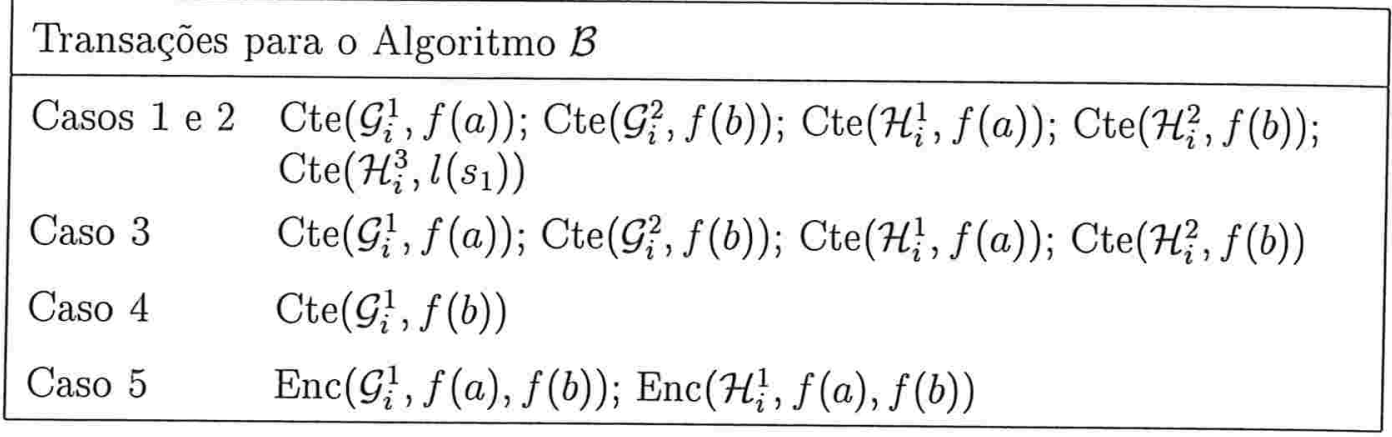

Tabela 4.2: Transações para o Algoritmo $\mathcal{B}$.

O fato de que o Algoritmo $\mathcal{B}$ elimina um arco de ligação por iteração é uma consequiência do Lema 4.5. Este lema nos permite afirmar que, em cada iteração Caso 1 ou Caso 2, ou um dos arcos incidentes em $f_{\mathcal{H}_{i}}(a)$ e $f_{\mathcal{H}_{i}}(b)$ é de ligação, ou então todas as hastes do eixo $(a, b)$ são de ligação. Portanto, cortando nestas iterações os arcos incidindo em $f_{\mathcal{H}_{i}}(a), f_{\mathcal{H}_{i}}(b)$ e mais uma das hastes, certamente o Algoritmo $\mathcal{B}$ elimina ao menos um arco de ligação por iteração de Casos 1 ou 2. As transações do Algoritmo $\mathcal{B}$ para os Casos 3, 4 e 5 são iguais às do Algoritmo $\mathcal{A}_{\bar{k}}$.

É digno de nota que a análise da razão de aproximação do Algoritmo $\mathcal{B}$ seja muito mais simples do que a do Algoritmo $\mathcal{A}_{2}$, cuja razão também é 3 . A análise que fizemos para o algoritmo $\mathcal{A}_{\bar{k}}, \bar{k} \geq 1$, embora seja um tanto longa e complicada, tem a vantagem de englobar o Algoritmo $\mathcal{A}_{1}$ (devido a Hein et al. $[19,20]$ ), que carecia de um resultado a respeito de seu desempenho, e também englobar o Algoritmo $\mathcal{A}_{2}$, colocando-os sub um mesmo foco. Embora os Algoritmos $\mathcal{A}_{2}$ e $\mathcal{B}$ tenham a mesma razão de aproximação, conforme veremos adiante, em experimentos com implementações de ambos os algoritmos observamos que o Algoritmo $\mathcal{A}_{2}$ parece produzir sistematicamente soluções com tamanho menor do que as soluções produzidas pelo Algoritmo $\mathcal{B}$. 


\section{Capítulo 5}

\section{Experimentos computacionais}

Este capítulo descreve a implementação dos algoritmos vistos no Capítulo 4, e mostra resultados de alguns testes feitos com essas implementações.

\subsection{O programa e formato dos arquivos de entrada e saída}

Os algoritmos vistos nos capítulos anteriores foram implementados em um programa, denominado MAF. Este programa lê um arquivo de entrada contendo a descrição de duas árvores filogenéticas com conjunto de rótulos iguais, e constrói uma floresta de concordância destas duas árvores empregando um dos algoritmos descritos no Capítulo 4. O usuário pode especificar o algoritmo de construção da floresta de concordância a ser aplicado, bem como a informação a ser fornecida pelo programa na saída, usando opções de linha de comando. Uma lista completa dessas opções pode ser obtida executando-se maf -h na linha de comando.

Os arquivos de entrada aceitos pelo programa MAF são arquivos ASCII contendo a descrição das árvores em um formato compatível com o padrão Newick de representação de árvores filogenéticas, que é utilizado pelo programa de manipulação de filogenias PHYLIP.

Uma descrição do padrão Newick pode ser encontrada em http://evolution.genetics . washington.edu/phylip/newicktree.html.

Os arquivos de saída produzidos por MAF empregam o mesmo padrão usado nos arquivos de entrada com uma modificação, definida neste trabalho, que permite representar componentes de restrições em árvores filogenéticas. Vamos descrever a seguir os formatos da entrada e da saída do programa MAF. 


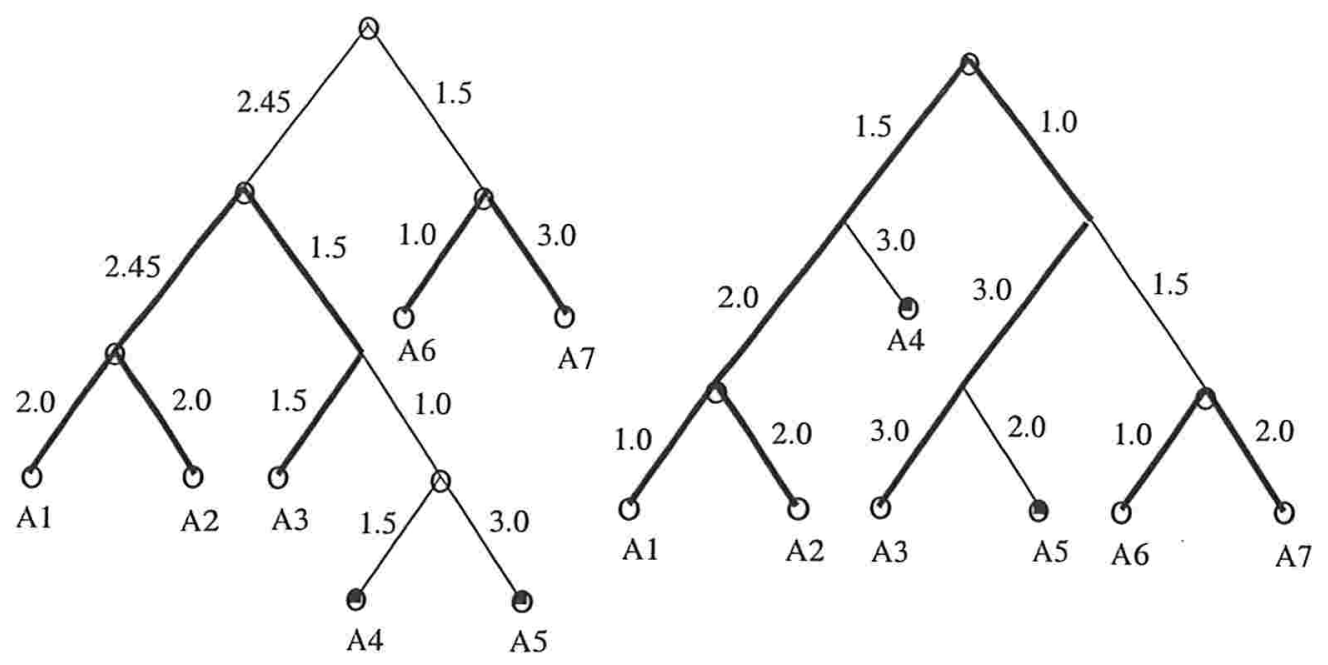

Figura 5.1: Exemplo da Figura 3.3 com comprimentos nos arcos.

O padrão que utilizamos para o arquivo de entrada emprega a correspondência entre árvores rotuladas e listas representadas por pares de parênteses aninhados. Nesse padrão, as folhas de uma árvore rotulada são representadas por cadeias de caracteres, sendo que o programa MAF trunca cadeias com mais do que 10 caracteres. Essas cadeias podem ter caracteres alfabéticos (maiúsculos ou minúsculos), dígitos e mais o caractere _, e devem começar por um caractere alfabético. Os nós internos da árvore são representados pela lista das representações dos seus descendentes imediatos. Essas representações são separadas por vírgulas, e a lista é colocada entre parênteses. Ao final, acrescenta-se um ponto-e-vírgula à representação completa da árvore. Observe que uma árvore pode ter mais de uma representação possível, pois a ordem dos filhos dos nós internos não é fixa (ou seja, a árvore não é ordenada).

O arquivo de entrada do programa MAF para as árvores da Figura 5.1, sem os comprimentos de arcos indicados na figura, é mostrado abaixo:

Arquivo 1:

$(((A 1, A 2),(A 3,(A 4, A 5))),(A 6, A 7))$;

$(((A 1, A 2), A 4),((A 3, A 5),(A 6, A 7)))$;

Nesta representação, podemos adicionalmente especificar para cada arco $e$ um comprimento $c_{e}$. Isto é feito acrescentando-se o símbolo ':' seguido de $c_{e}$ logo depois da representação da extremidade inferior do arco e. No exemplo acima, o arquivo de entrada incluindo os comprimentos dos arcos poderia ser: 


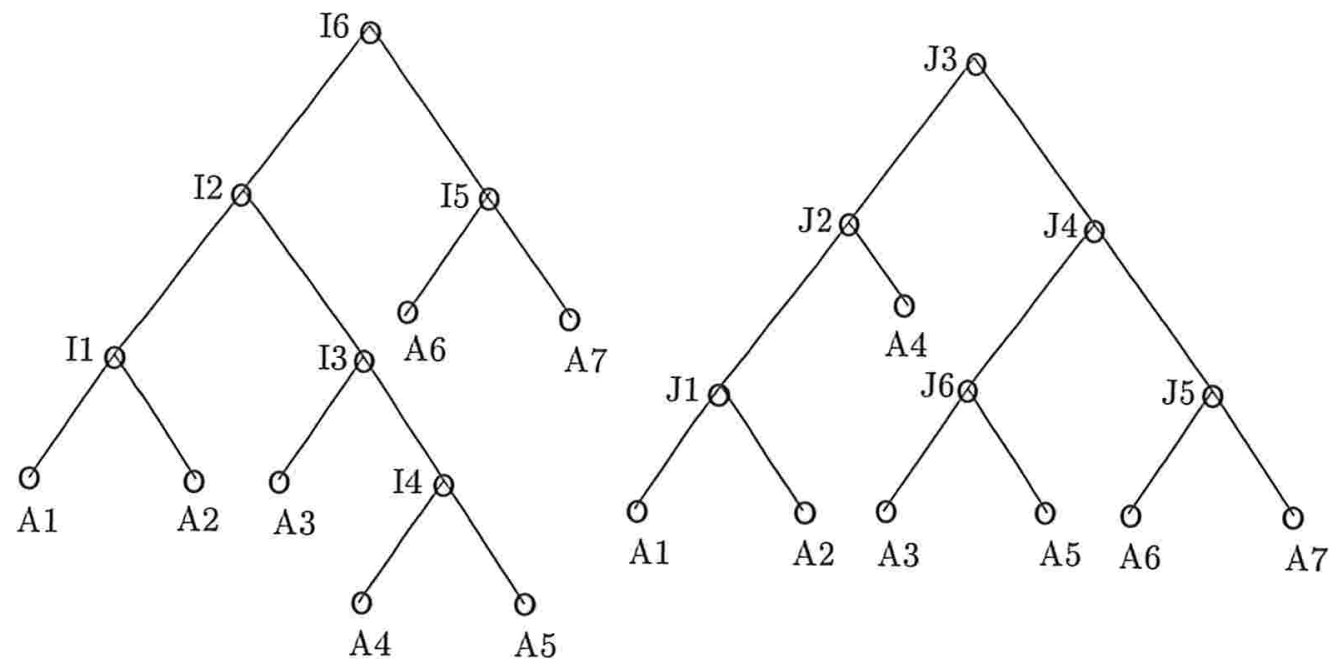

Figura 5.2: Exemplo da Figura 3.3 com rótulos nos nós internos.

Arquivo 2:

$(((A 1: 2.0, A 2: 2.0): 2.45$

$(\mathrm{A} 3: 1.5,(\mathrm{~A} 4: 1.5, \mathrm{~A} 5: 3.0): 1.0): 1.5): 2.45, \quad(\mathrm{~A} 6: 1.0, \mathrm{~A} 7: 3.0): 1.5)$;

$(((\mathrm{A} 1: 1.0, \mathrm{~A} 2: 2.0): 2.0, \mathrm{~A} 4: 3.0): 1.5$,

$((A 3: 3.0, A 5: 2.0): 3.0,(A 6: 1.0, A 7: 2.0): 1.5): 1.0)$

Nós internos podem também ter rótulos, conforme a Figura 5.2 exemplifica. O arquivo de entrada para as árvores nesta figura poderia ser:

Arquivo 3:

$(((A 1, A 2) I 1,(A 3,(A 4, A 5) I 4) I 3) I 2,(A 6, A 7) I 5) I 6$;

( ( (A1, A2) J1, A4) J2, ( (A3, A5) J6, (A6, A7) J5) J4) J3;

Tanto os rótulos dos nós internos quanto os comprimentos dos arcos, quando presentes no arquivo de entrada, são ignorados pelos algoritmos de construção de florestas de concordância implementados em MAF. Rótulos nos nós internos, quando presentes, são impressos na saída.

As florestas de concordância construídas internamente pelo programa podem ser mostradas para o usuário na saída do programa com a opção de linha de comando $-r$. Neste caso, o programa imprime na saída as árvores dadas como entrada no formato apresentado acima, com as raízes dos componentes da floresta obtida precedidas por asteriscos. Abaixo, mostramos a saída que o programa obtém para o exemplo da 
Figura 5.1 com os Arquivos 1 ou 2. A floresta de concordância representada por essa saída está indicada em linhas escuras na Figura 5.1.

$$
\begin{aligned}
& (*(A 6, A 7), *((A 1, A 2),((* A 5, * A 4), A 3))) ; \\
& *((* A 4,(A 1, A 2)),(*(A 6, A 7),(* A 5, A 3))) ;
\end{aligned}
$$

Observamos que este formato utilizado nos arquivos de saída de MAF não é compatível com o padrão Newick devido à presença dos asteriscos.

Para facilitar a visualização das árvores e das florestas de concordância representadas no padrão que descrevemos aqui, escrevemos um utilitário chamado PRINTTREE, que lê da entrada padrão um arquivo com duas árvores nesse formato, e imprime na saída padrão diagramas dessas árvores. Esse utilitário aceita arquivos contendo rótulos nos nós internos, comprimentos nos arcos e asteriscos marcando componentes de restrições. Nas Figuras 5.3, 5.4 e 5.5, mostramos a saída desse utilitário para os arquivos exibidos nesta seção.

\subsection{Estrutura de dados para florestas filogenéticas}

Em nossas implementações dos algoritmos vistos neste trabalho, utilizamos estruturas de dados com apontadores para representar árvores e florestas filogenéticas. Vamos considerar, para a exposição que faremos neste capítulo, que NIL representa um apontador distinto de quaisquer outros apontadores utilizados neste trabalho.

Se $\mathcal{G}$ é uma floresta filogenética, então consideramos uma ordem total sobre os componentes de $\mathcal{G}$ e, dentro de cada componente, ordenamos os descendentes imediatos de cada nó interno. Seja $m$ o número de componentes de $\mathcal{G}$, e $\mathcal{V}_{1}, \ldots, \mathcal{V}_{m}$ os componentes.

A floresta filogenética $\mathcal{G}$ possui os atributos raiz e $f$. O atributo raiz(G) é um nó distinto de NIL e dos nós de $\mathcal{G}$, e $f(\mathcal{G})$ é um vetor de nós de $\mathcal{G}$ representando a bijeção $f_{\mathcal{G}}$. Sobre o conjunto de rótulos de $\mathcal{G}$ é estabelecida uma ordem total, e cada rótulo é referenciado pela sua posição nesta ordem, que chamamos de índice do rótulo. $\mathrm{O}$ vetor $f(\mathcal{G})$ é indexado por esses índices.

Se $\mathcal{T}$ e $\mathcal{U}$ são as árvores dadas como entrada para o programa MAF, então estabelecemos sobre $S_{\mathcal{T}}$ e $S_{\mathcal{U}}$ a ordem total induzida pela pré-ordem de $\mathcal{T}$. Essa convenção facilita a localização de pares de folhas irmãs pelo método básico, conforme veremos adiante.

Os nós de $\mathcal{G}$ mais o nó $\operatorname{raiz}(\mathcal{G})$ possuem os atributos pai, grau, distância, rótulo, predecessor e sucessor.

Se $x$ é um nó de $\mathcal{G}$, então: 
Arquivo 1:

$(((A 1, A 2),(A 3,(A 4, A 5))),(A 6, A 7))$;

$(((A 1, A 2), A 4),((A 3, A 5),(A 6, A 7)))$;

Arquivo 2:

$(((\mathrm{A} 1: 2.0, \mathrm{~A} 2: 2.0): 2.45,(\mathrm{~A} 3: 1.5,(\mathrm{~A} 4: 1.5, \mathrm{~A} 5: 3.0): 1.0): 1.5): 2.45$, $(A 6: 1.0, A 7: 3.0): 1.5)$

$(((A 1: 1.0, A 2: 2.0): 2.0, A 4: 3.0): 1.5$,

$((A 3: 3.0, A 5: 2.0): 3.0,(A 6: 1.0, A 7: 2.0): 1.5): 1.0)$;

Saída do PRINTTREE:
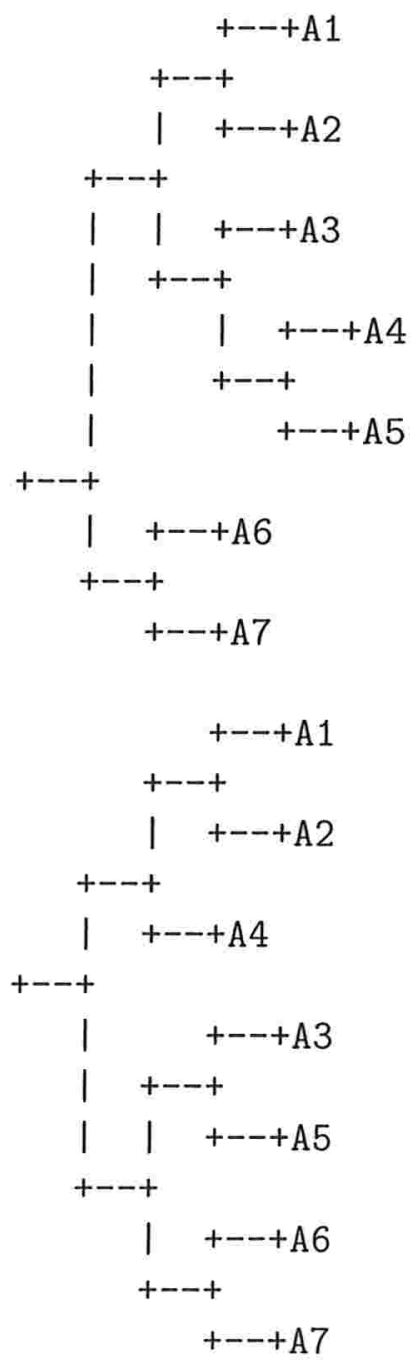

Figura 5.3: Saída do programa PRINTTREE para os Arquivos 1 e 2. 
Arquivo 3:

( ( (A1, A2)I1, (A3, (A4, A5) I4) I3)I2, (A6, A7) I5) I6 ;

( ( (A1, A2) J1, A4) J2, ( (A3, A5) J6, (A6, A7) J5) J4) J3;

Saída do PRINTTREE:

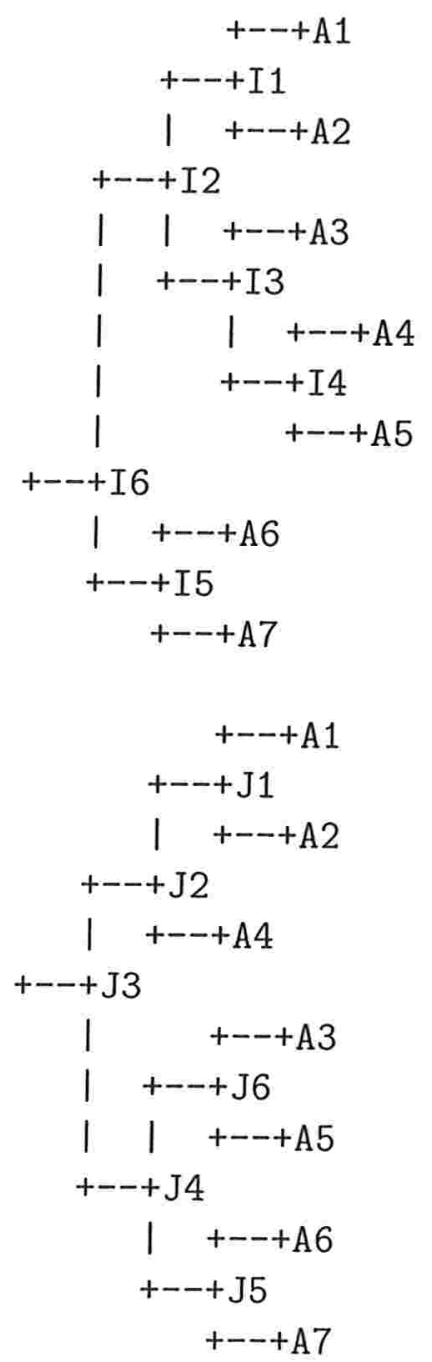

Figura 5.4: Saída do programa PRINTTREE para o Arquivo 3. 
Arquivo 4:

$(*(\mathrm{~A} 6, \mathrm{~A} 7), *((\mathrm{~A} 1, \mathrm{~A} 2),((* A 5, * A 4), \mathrm{A} 3)))$;

$*((* \mathrm{~A} 4,(\mathrm{~A} 1, \mathrm{~A} 2)),(*(\mathrm{~A} 6, \mathrm{~A} 7),(* \mathrm{~A} 5, \mathrm{~A} 3)))$;

Saída do PRINTTREE:
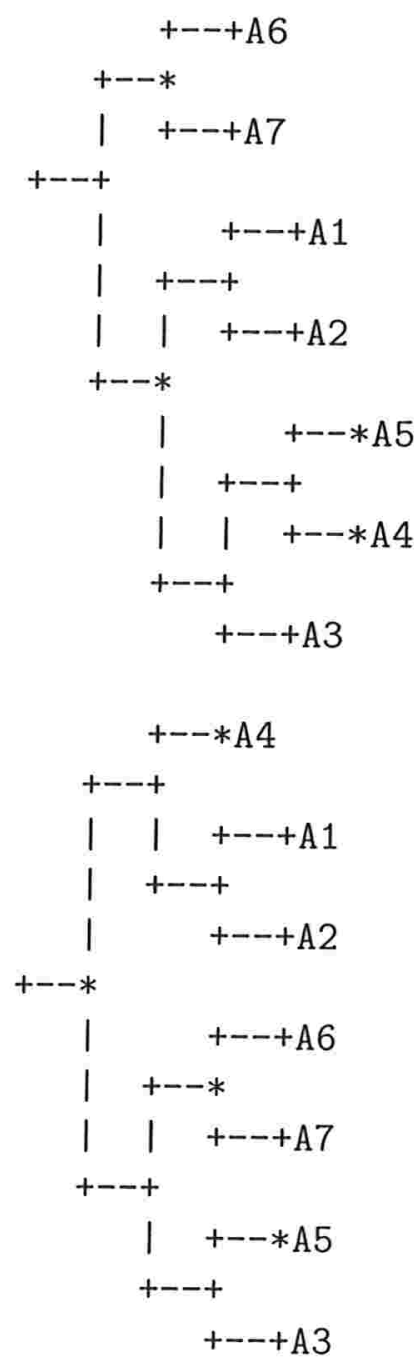

Figura 5.5: Saída do programa PRINTTREE para o Arquivo 4. 
- pai(x) é o nó antepassado imediato de $x$ se $x$ não é raiz de um componente de $\mathcal{G}$, caso contrário $\operatorname{pai}(x)$ é o nó $\operatorname{raiz}(\mathcal{G})$;

- $\operatorname{grau}(x)$ é o grau de $x$ em $\mathcal{G}$;

- distância $(x)$ é 1 mais a distância de $x$ à raiz do componente de $\mathcal{G}$ que contém $x$;

- rótulo $(x)$ é o rótulo de $x$ se $x$ é uma folha, caso contrário rótulo $(x)$ é NIL;

- predecessor $(x)$ e sucessor $(x)$ são respectivamente o nó predecessor e o nó sucessor de $x$ na pré-ordem induzida pela ordenação que adotamos para $\mathcal{G}$. Para $i=$ $1, \ldots, m-1$, o predecessor de $r_{\mathcal{V}_{i+1}}$ é o último nó na pré-ordem de $\mathcal{V}_{i}$, e o sucessor do último nó na pré-ordem de $\mathcal{V}_{i}$ é $r_{\mathcal{V}_{i+1}}$; o predecessor de $r_{\mathcal{V}_{1}}$ e o sucessor do último nó na pré-ordem de $\mathcal{V}_{m}$ são definidos como $\operatorname{raiz}(\mathcal{G})$.

Se $x=\operatorname{raiz}(\mathcal{G})$, então:

- $\operatorname{pai}(x)=\mathrm{NIL}$;

- $\operatorname{grau}(x)=m$;

- $\operatorname{distância}(x)=0$;

- $\operatorname{rótulo}(x)=$ NIL;

- $\operatorname{predecessor}(x)$ é o último nó na pré-ordem de $\mathcal{V}_{m}$;

- $\operatorname{sucessor}(x)=r_{\mathcal{V}_{1}}$.

Esta estrutura de dados é semelhante à estrutura que é utilizada em implementações do Método Simplex para Redes (ver Chvátal [9]) para guardar a árvore geradora induzida pelos arcos na base. A semelhança aparece no emprego dos atributos pai, distância, predecessor e sucessor. Conforme veremos, esta estrutura é conveniente para a localização de eixos e contagem de hastes em restrições, na determinação dos casos em cada iteração dos Algoritmos $\mathcal{A}_{\bar{k}}$ e $\mathcal{B}$, e também na reconstrução das restrições induzidas pela floresta de concordância obtida sobre as árvores da entrada.

A Figura 5.6 esquematiza um exemplo desta estrutura para os componentes da floresta de concordância mostrada na Figura 5.1. Na figura estão mostrados entre parênteses os atributos grau, distância e rótulo para cada nó da floresta e também para o atributo raiz desta floresta. Rótulos não especificados são indicados por NIL. Os valores do atributo pai para os nós da floresta que não são raízes de componentes estão indicados por setas simples e para as raízes em setas tracejadas. Os atributos predecessor e sucessor estão indicados por setas pontilhadas. Os atributos raiz e $f$ da árvore estão representados por setas tracejadas. 


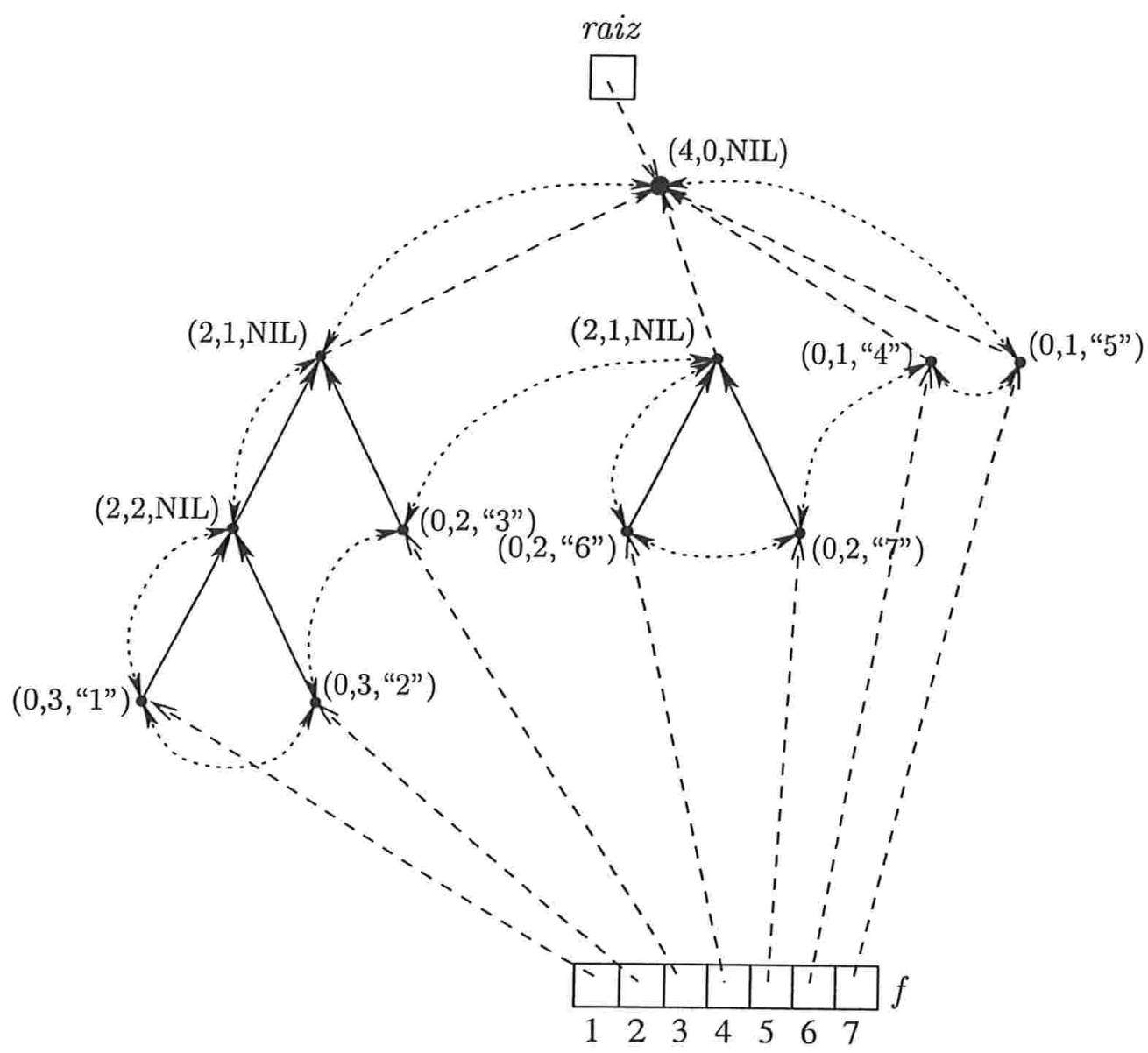

Figura 5.6: Representação interna da floresta de concordância na Figura 5.1. 


\subsection{Implementação dos algoritmos}

Seja $\mathcal{G}$ uma floresta filogenética. Os procedimentos básicos implementados pelo programa MAF, que compõem as implementações dos algoritmos do Capítulo 4, executam as seguintes operações sobre uma estrutura de dados que armazene $\mathcal{G}$ :

1) A busca por um par de folhas irmãs $f_{\mathcal{G}}(a), f_{\mathcal{G}}(b)$ com $a, b \in S_{\mathcal{G}}$.

2) A localização do lca do par de nós $f_{\mathcal{G}}(a)$ e $f_{\mathcal{G}}(b)$ tais que $a, b \in S_{\mathcal{G}}$.

3) A movimentação, na estrutura de dados, de uma restrição simples $\mathcal{G} \mid D(x)$ tal que $x$ é um nó de $\mathcal{G}$ para um local na estrutura indicado por um nó $y$. Especificamente, o nó $x$ passa a ser um descendente imediato, na estrutura de dados, do nó $y$, e também o o sucessor deste na pré-ordem da estrutura. Observe que podemos ter $y=\operatorname{raiz}(\mathcal{G})$.

4) A contração de um arco $s(x)$ tal que $x$ é um nó de $\mathcal{G}$ com grau 1 .

5) A extensão de um nó $x$ de $\mathcal{G}$ (esta operação restaura a situação anterior em $x$ contraído).

A primeira operação é executada sobre a floresta $\mathcal{G}_{i}$ para toda iteração $i$ dos algoritmos de construção de florestas de concordância. Como a ordem dos nós no vetor $f\left(\mathcal{G}_{i}\right)$ é induzida pela pré-ordem de $\mathcal{T}$, então podemos implementar esta operação em tempo linear no número de folhas da floresta $\mathcal{G}_{i}$, conforme explicamos a seguir.

Inicialmente, o vetor $f\left(\mathcal{G}_{i}\right)$ é percorrido na ordem crescente dos índices em busca de um nó que não esteja isolado. Se todos os nós em $f\left(\mathcal{G}_{i}\right)$ estão isolados, então não existem pares de folhas irmãs em $\mathcal{G}_{i}$ e o procedimento pára devolvendo um valor que indique esta condição. Se existe um nó $x$ em $f\left(\mathcal{G}_{i}\right)$ que não está isolado, então o procedimento verifica se sucessor $(x)$ é uma folha. Se ele é, então $x$ e o seu sucessor formam um par de folhas irmãs, e o procedimento pára, devolvendo esses dois nós. Se sucessor $(x)$ não é uma folha, então o procedimento testa o nó seguinte no vetor $f\left(\mathcal{G}_{i}\right)$.

A operação (2) é executada sobre a floresta $\mathcal{H}_{i}$. Durante a sua execução, fazse a localização do eixo $(a, b)$ em $\mathcal{H}_{i}$ e a contagem das hastes, ambas necessárias para a determinação do caso que se aplica em cada iteração. Esta operação pode ser facilmente implementada em tempo linear no número de arcos de $\mathcal{U}$ usando-se a informação dada pelos atributos pai e distância para a localização do lca e grau para a contagem das hastes.

A operação (3) pode ser implementada em tempo linear no número de arcos de $\mathcal{T}$ e $\mathcal{U}$. Essa implementação executa a seguinte seqüência ações: ajuste dos atributos $\operatorname{grau}(y)$ e grau $(\operatorname{pai}(x))$; atribuição de $y$ ao atributo pai $(x)$; ajuste do atributo 
distância para todos os nós descendentes de $x$ em $\mathcal{G}$; e ajuste da lista de pré-ordem implementada pelos atributos predecessor e sucessor. O terceiro e o quarto destes passos requerem tempo linear no número de nós de $\mathcal{T}$ ou $\mathcal{U}$ para serem executados, enquanto os demais passos requerem tempo constante.

A operação de movimentação é usada para implementar as operações de remoção e conexão. A operação de remoção de um arco $e$ de $\mathcal{G}$ equivale à movimentação da restrição simples $\mathcal{G} \mid D_{e}$ para o nó $\operatorname{raiz}(\mathcal{G})$. Observe que, com isso, a nova estrutura de dados representa a restrição $\mathcal{G} \mid D_{e}$ como um novo componente, e o atributo grau $(\operatorname{raiz}(\mathcal{G}))$ registra o novo número de componentes. A operação de conexão de um componente $\mathcal{W}$ de $\mathcal{G}$ através de um nó $x$ de $\mathcal{G}$ não contido em $\mathcal{W}$ é implementada pela movimentação do componente $\mathcal{W}$ (cuja raiz é descendente imediata de $\operatorname{raiz}(\mathcal{G})$ na estrutura de dados de $\mathcal{G}$ ) para o nó $x$.

As operações (4) e (5) são implementadas pela seguinte seqüência de ações sobre a estrutura de dados de $\mathcal{G}$ : alteração do atributo pai do nó $x$; ajuste do atributo distância para todos os nós descendentes de $x$ em $\mathcal{G}$; e ajuste da lista de pré-ordem. Da mesma forma que ocorre com a operação de movimentação, as etapas de ajuste de distância e da pré-ordem requerem tempo linear.

A implementação das operações de eliminação, inserção e transferência de subárvore usando as operações (1) a (5) é imediata. Em particular, implementamos transferências de subárvore utilizando movimentações, ao invés de utilizarmos eliminações e inserções.

Ao término da execução dos algoritmos de construção de florestas de concordância, as florestas $\mathcal{G}_{i}$ e $\mathcal{H}_{i}$ são triviais, conforme vimos no Capítulo 4. Para que as restrições das florestas de concordância construídas possam ser exibidas para o usuário do programa MAF, seus componentes precisam ser reconstruídos para serem posteriormente impressos na saída, usando o padrão que especificamos na Seção 5.1. O programa executa esta tarefa a partir de informação adicional que é armazenada quando as operações de corte e encolhimento são executadas. O espaço necessário para essa informação adicional é linear no número de folhas das árvores dadas como entrada.

\subsection{Testes e o algoritmo de aproximação $\mathcal{C}$}

Foram implementados neste trabalho os seguintes algoritmos para o problema MAF-2 descritos no Capítulo 4: o Algoritmo $\mathcal{A}_{1}$, o Algoritmo $\mathcal{A}_{2}$ e o Algoritmo $\mathcal{B}$. Adicionalmente, fizemos testes com um quarto algoritmo, chamado Algoritmo $\mathcal{C}$, que é uma variante do Algoritmo $\mathcal{B}$, conforme explicamos a seguir.

O Algoritmo $\mathcal{C}$, assim como os Algoritmos $\mathcal{A}_{\bar{k}}$ e $\mathcal{B}$, utiliza o método básico, e suas transações estão listadas na Tabela 5.1. As transações para os Casos 1, 2, 4 e 5 


\begin{tabular}{|ll|}
\hline \multicolumn{2}{|c|}{ Transações para o Algoritmo $\mathcal{C}$} \\
\hline Casos 1 e 2 & $\operatorname{Cte}\left(\mathcal{G}_{i}^{1}, f(a)\right) ; \operatorname{Cte}\left(\mathcal{G}_{i}^{2}, f(b)\right) ; \operatorname{Cte}\left(\mathcal{H}_{i}^{1}, f(a)\right) ; \operatorname{Cte}\left(\mathcal{H}_{i}^{2}, f(b)\right) ;$ \\
& $\operatorname{Cte}\left(\mathcal{H}_{i}^{3}, l\left(s_{1}\right)\right)$ \\
Caso 3 & $\operatorname{Cte}\left(\mathcal{G}_{i}^{1}, f(a)\right) ; \operatorname{Cte}\left(\mathcal{G}_{i}^{2}, f(b)\right) ; \operatorname{Cte}\left(\mathcal{H}_{i}^{1}, f(a)\right) ; \operatorname{Cte}\left(\mathcal{H}_{i}^{2}, f(b)\right) ;$ \\
& $\operatorname{Cte}\left(\mathcal{H}_{i}^{3}, f(c)\right)$ \\
Caso 4 & $\operatorname{Cte}\left(\mathcal{G}_{i}^{1}, f(b)\right)$ \\
Caso 5 & $\operatorname{Enc}\left(\mathcal{G}_{i}^{1}, f(a), f(b)\right) ; \operatorname{Enc}\left(\mathcal{H}_{i}^{1}, f(a), f(b)\right)$ \\
\hline
\end{tabular}

Tabela 5.1: Transações para o Algoritmo $\mathcal{C}$.

são as mesmas do Algoritmo $\mathcal{B}$. A transação do Caso 3 difere daquela empregada no Algoritmo $\mathcal{B}$.

No Algoritmo $\mathcal{C}$, se ocorre o Caso 3 na iteração $i$ e $\mathcal{H}_{i}$ admite uma terceira folha além de $f_{\mathcal{H}_{i}}(a)$ e $f_{\mathcal{H}_{i}}(a)$, então a respectiva transação corta, além dos $\operatorname{arcos} s\left(f_{\mathcal{H}_{i}}(a)\right)$ e $s\left(f_{\mathcal{H}_{i}}(b)\right)$, o arco $s\left(f_{\mathcal{H}_{i}}(c)\right)$, sendo $c$ um rótulo em $S_{\mathcal{H}_{i}}$ diferente de $a$ e $b$.

Não é difícil ver que o Algoritmo $\mathcal{C}$ é uma 3-aproximação para o problema MAF-2: suas transações satisfazem a propriedade de que em cada iteração pelo menos um arco de ligação é eliminado, e o número máximo de cortes por iteração é 3, como no Algoritmo $\mathcal{B}$.

Nossa motivação para desenvolver e implementar o Algoritmo $\mathcal{C}$ foi prover bons limitantes inferiores para o valor da solução ótima das instâncias com que testamos as implementações de $\mathcal{A}_{1}, \mathcal{A}_{2}$ e $\mathcal{B}$. A idéia é que se tivermos à disposição um algoritmo de 3-aproximação para o problema MAF-2 que construa para cada instância deste problema uma floresta de concordância cujo tamanho fique próximo de três vezes o tamanho de uma floresta de concordância ótima, então podemos obter limitantes inferiores de boa qualidade para este tamanho ótimo dividindo por três o tamanho das soluções viáveis produzidas por este algoritmo. Nos testes que fizemos com as implementações de $\mathcal{A}_{1}, \mathcal{A}_{2}, \mathcal{B}$ e $\mathcal{C}$, o Algoritmo $\mathcal{C}$ produziu consistentemente soluções de qualidade pior do que a dos demais algoritmos. Assim, o Algoritmo $\mathcal{C}$ forneceu os melhores limitantes inferiores para o valor da solução ótima das instâncias do problema MAF-2 que testamos.

Seja $\mathcal{X}$ qualquer um dos algoritmos $\mathcal{A}_{2}, \mathcal{B}$ ou $\mathcal{C}$. Sabemos que, para cada instância $\mathcal{T}, \mathcal{U}$ de $M A F-2$, vale:

(valor devolvido pelo Algoritmo $\mathcal{X})-1 \leq 3(\mid$ solução ótima para $\mathcal{T}$ e $\mathcal{U} \mid-1)$.

Portanto, temos que:

$$
l_{\mathcal{X}}:=\lceil((\text { valor devolvido pelo Algoritmo } \mathcal{X})+2) / 3\rceil
$$


é um limitante inferior para o valor da solução ótima do problema MAF-2.

Foi desenvolvido um utilitário, chamado SCRAMBLE, para gerar as instâncias que utilizamos nos testes relatados nesta seção. Esse utilitário recebe como entradas através de opções de linha de comando o número de folhas desejadas na instância, um valor inicial para o gerador de números aleatórios (opcional) e o número de transferências de subárvore a serem executadas na geração de instâncias. Uma lista completa das opções de linha de comando disponíveis pode ser obtida executando-se scramble -h na linha de comando. A saída do programa é um arquivo ASCII contendo um par de árvores no formato descrito na Seção 5.1 para os arquivos de entrada do programa MAF.

O algoritmo utilizado por SCRAMBLE inicialmente lê um par de árvores filogenéticas de um arquivo no padrão descrito na Seção 5.1, ou, caso o usuário prefira, gera uma árvore aleatoriamente e faz uma cópia dessa árvore para compor a segunda árvore do par. A opção para leitura ou geração de árvores pode ser especificada através da linha de comando. Caso o usuário prefira que o programa gere as árvores, o seguinte algoritmo é usado para criar a primeira árvore do par.

Inicialmente, uma árvore filogenética com um único nó é criado. A partir daí, executa-se $n-1$ vezes a seguinte seqüência de operações, sendo $n$ o número de folhas especificado pelo usuário. Sorteia-se um nó da árvore que está sendo construída e estende-se esse nó. Com isso, um novo nó de grau 1 é criado na árvore. Em seguida, cria-se um novo nó, que é conectado a esse nó de grau 1. A escolha do nó a ser estendido é feita sorteando-se um inteiro $p$ de 1 a $2 j-1$, sendo $j$ o número de folhas na árvore $(1 \leq j<n)$, e em seguida tomando-se o $(p-1)$-ésimo sucessor da raiz dessa árvore em pré-ordem.

Se o programa lê um par de árvores da entrada, ele imprime a primeira árvore desse par na saída; caso ele gere uma árvore aleatoriamente, ele imprime essa árvore na saída. Em seguida, o programa executa sobre a árvore gerada aleatoriamente, ou sobre a segunda árvore lida da entrada, uma seqüência de $t$ operações de transferência de subárvore escolhidas aleatoriamente, sendo $t$ o número de transferências especificado pelo usuário. A árvore resultante é, então, impressa na saída, compondo assim, junto com a árvore impressa anteriormente, uma entrada para o programa MAF. As operações de transferência são especificadas escolhendo-se aleatoriamente dois nós da árvore, $x$ e $y$, tais que $x$ não é a raiz da árvore e $y$ não é o antepassado imediato de $x$. A escolha aleatória desses nós é feita como na escolha dos nós no algoritmo de geração de árvores, visto acima ${ }^{1}$.

Para executar os testes aqui relatados, geramos com o SCRAMBLE dez arquivos de entrada para o programa MAF contendo instâncias com valor ótimo 1 (isto é, com duas árvores isomorfas em cada arquivo) e 1000 folhas em cada árvore. Denominamos estes arquivos de 001-001, 002-001, .., 010-001. Em seguida, aplicamos à segunda

\footnotetext{
${ }^{1} \mathrm{~A}$ pré-ordem é armazenada internamente em uma lista ligada circular.
} 
árvore de cada um desses arquivos uma seqüência de 4 operações de transferência de subárvore, formando com isso uma segunda família de arquivos, os quais denominamos 001-005, 002-005, ..., 010-005. Aplicando sucessivamente o mesmo procedimento a partir destes arquivos, obtivemos os arquivos

$$
\begin{aligned}
& \text { 001-010, ., 010-010, } \\
& 001-020, \ldots, 010-020, \\
& 001-050, \ldots, 010-050, \\
& 001-100, \ldots, 010-100, \\
& 001-200, \ldots, 010-200, \\
& 001-300, \ldots, 010-300, \mathrm{e} \\
& 001-400, \ldots, 010-400,
\end{aligned}
$$

aplicando seqüências de respectivamente 5, 10, 30, 50, 100, 100 e 100 transferências de subárvore.

Os resultados obtidos executando-se MAF com cada um desses arquivos estão listados nas Tabelas 5.2, 5.3, 5.4 e 5.5. A razão calculada na coluna (Alg. $\left.\mathcal{A}_{2}\right) / l_{\mathcal{C}}$ exibe um limitante superior para a razão de desempenho da solução fornecida por $\mathcal{A}_{2}$ (ver Seção 2.4). A última coluna da tabela exibe os limitantes inferior $\left(l_{\mathcal{C}}\right)$ e superior $(t+1$, sendo $t$ o número de transferências de subárvore usadas para gerar a instância) para o valor ótimo de cada instância.

As seguintes observações podem ser feitas a respeito destas tabelas:

- $\mathrm{O}$ algoritmo $\mathcal{A}_{2}$ produz sistematicamente soluções de tamanho igual ou menor do que as soluções produzidas pelo Algoritmo $\mathcal{A}_{1}$. As soluções dadas pelo Algoritmo $\mathcal{A}_{2}$ são consistentemente melhores (têm menor número de componentes) quando o tamanho da solução ótima é maior ou igual a 100 componentes para árvores com 1000 folhas.

- Podemos ver, pela coluna $\left(\mathrm{Alg} . \mathcal{A}_{2}\right) / l_{\mathcal{C}}$ de cada tabela, que o Algoritmo $\mathcal{A}_{2}$ produz soluções com tamanho menor ou igual a 2 vezes o valor da solução ótima quando o tamanho da solução ótima é menor ou igual a 200 componentes em árvores com 1000 folhas. Esta razão aproxima-se de 1,5 à medida que o tamanho da solução ótima diminui.

- Se a razão entre o valor da solução ótima e o número de rótulos da instância é menor ou igual a $0,5 \%$, o Algoritmo $\mathcal{C}$ permite determinar o valor da solução ótima. (Na Tabela 5.2, este valor é 5 .)

Destas observações, concluímos que, dentre os quatro algoritmos de aproximação que implementamos para o problema MAF-2 (sendo três deles de 3-aproximação e um de 4-aproximação), o Algoritmo $\mathcal{A}_{2}$ é o que tem melhor desempenho com relação 


\begin{tabular}{|c|c|c|c|c|c|c|c|}
\hline \multicolumn{8}{|c|}{ Ótimo $\leq 5(t=4$ transferências de subárvore $) ; \mathrm{n}=1000$} \\
\hline $001-005$ & 5 & 5 & 13 & 13 & 5 & 1.00 & $5-5$ \\
\hline 002-005 & 8 & 8 & 13 & 13 & 5 & 1.60 & $5-5$ \\
\hline 003-005 & 6 & 6 & 13 & 13 & 5 & 1.20 & $5-5$ \\
\hline 004-005 & 7 & 7 & 13 & 13 & 5 & 1.40 & $5-5$ \\
\hline 005-005 & 7 & 7 & 13 & 13 & 5 & 1.40 & $5-5$ \\
\hline 006-005 & 7 & 7 & 13 & 13 & 5 & 1.40 & $5-5$ \\
\hline 007-005 & 7 & 7 & 13 & 13 & 5 & 1.40 & $5-5$ \\
\hline 008-005 & 8 & 8 & 13 & 13 & 5 & 1.60 & $5-5$ \\
\hline 009-005 & 8 & 8 & 13 & 13 & 5 & 1.60 & $5-5$ \\
\hline 010-005 & 8 & 8 & 13 & 13 & 5 & 1.60 & $5-5$ \\
\hline \multicolumn{8}{|c|}{ Ótimo $\leq 10(t=9$ transferências de subárvore $) ; \mathrm{n}=1000$} \\
\hline 001-010 & 15 & 15 & 28 & 28 & 10 & 1.50 & $10-10$ \\
\hline 002-010 & 15 & 15 & 28 & 28 & 10 & 1.50 & $10-10$ \\
\hline 003-010 & 16 & 16 & 28 & 28 & 10 & 1.60 & $10-10$ \\
\hline 004-010 & 15 & 15 & 27 & 28 & 10 & 1.50 & $10-10$ \\
\hline 005-010 & 13 & 13 & 28 & 28 & 10 & 1.30 & $10-10$ \\
\hline 006-010 & 16 & 16 & 28 & 28 & 10 & 1.60 & $10-10$ \\
\hline 007-010 & 16 & 16 & 28 & 28 & 10 & 1.60 & $10-10$ \\
\hline 008-010 & 14 & 14 & 25 & 25 & 9 & 1.56 & $9-10$ \\
\hline 009-010 & 13 & 13 & 24 & 25 & 9 & 1.45 & $9-10$ \\
\hline 010-010 & 13 & 13 & 28 & 28 & 10 & 1.30 & $10-10$ \\
\hline
\end{tabular}

Tabela 5.2: Testes com os Algoritmos $\mathcal{A}_{2}, \mathcal{A}_{1}, \mathcal{B}$ e $\mathcal{C}$ (parte 1). 


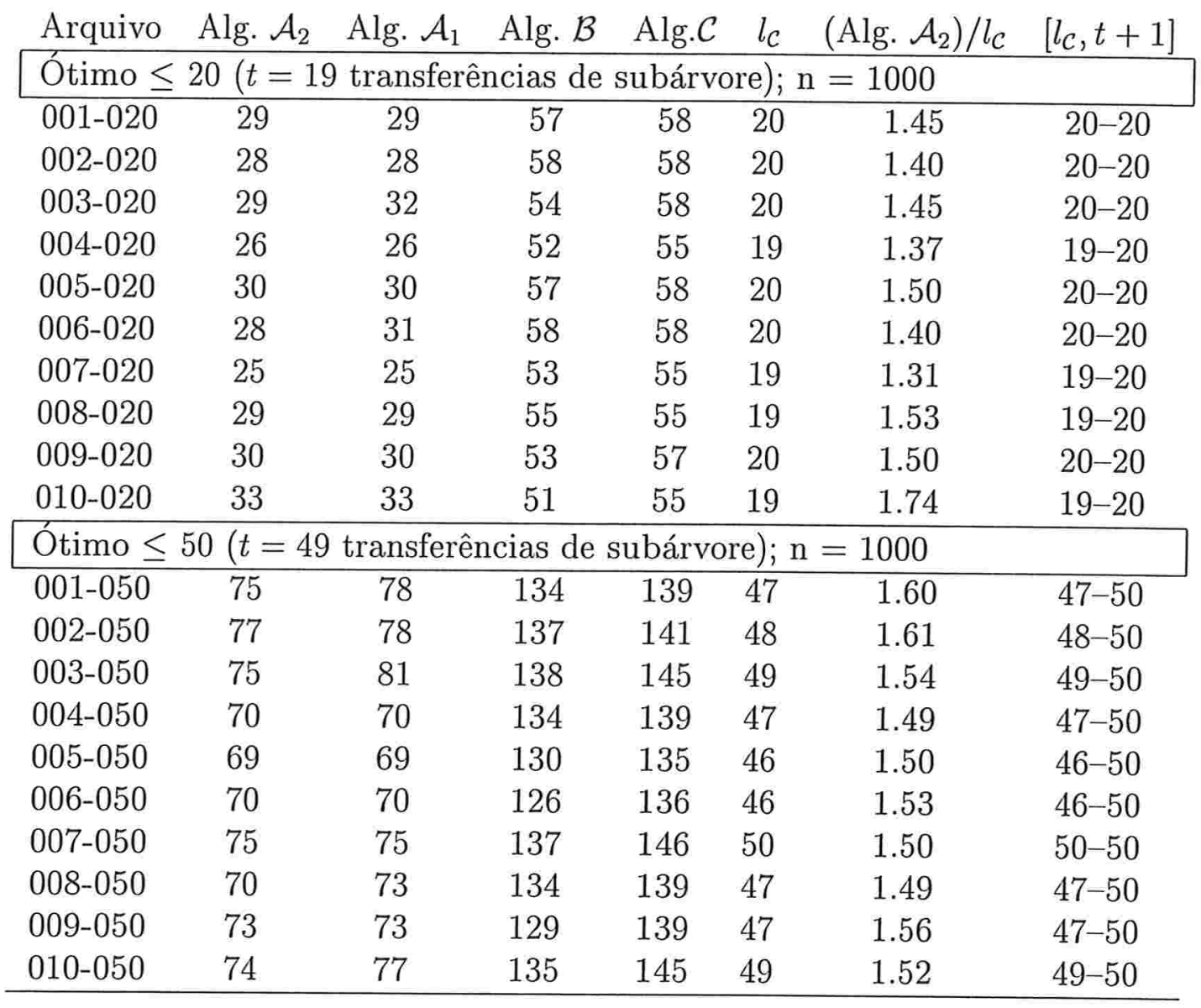

Tabela 5.3: Testes com os Algoritmos $\mathcal{A}_{2}, \mathcal{A}_{1}, \mathcal{B}$ e $\mathcal{C}$ (parte 2). 


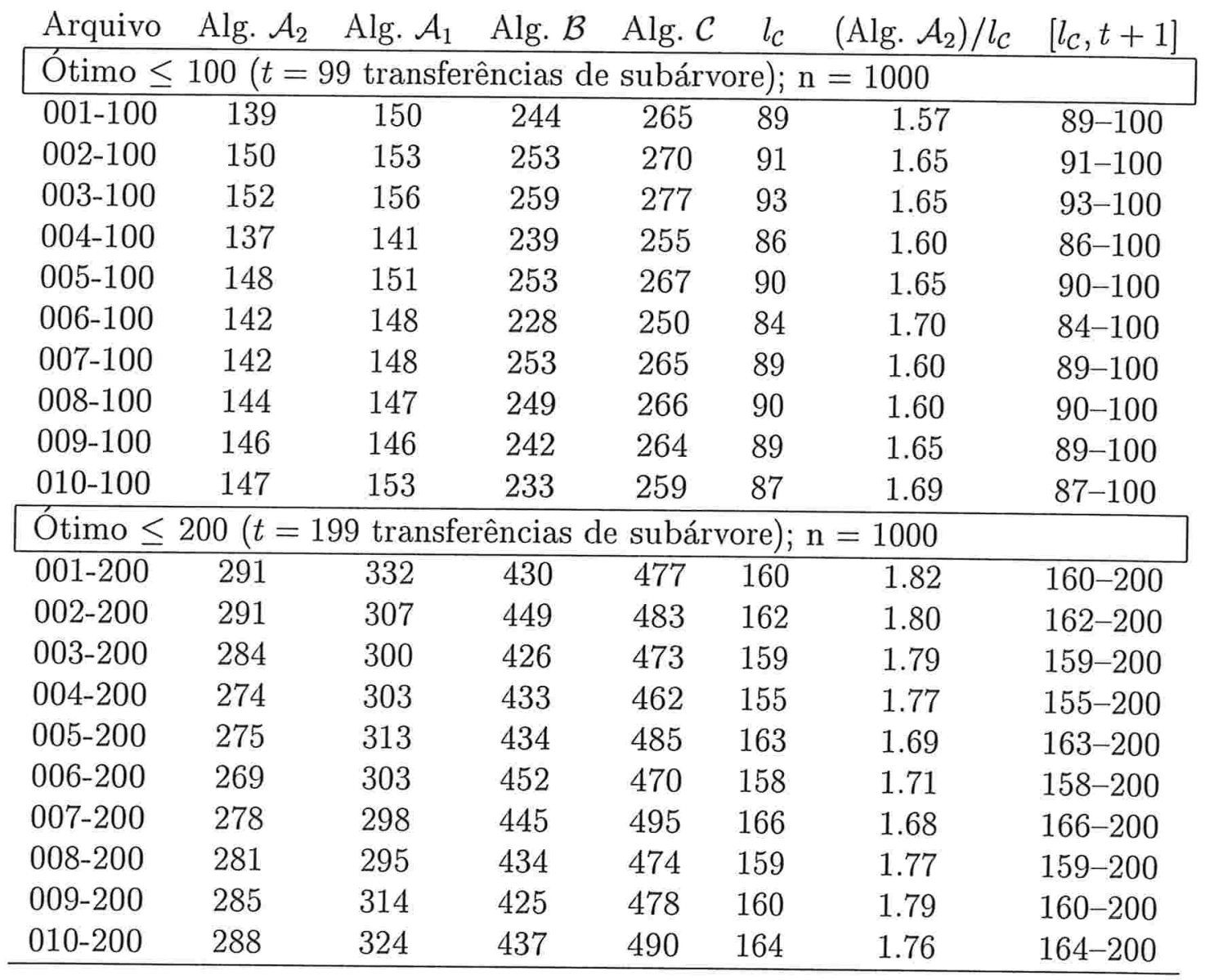

Tabela 5.4: Testes com os Algoritmos $\mathcal{A}_{2}, \mathcal{A}_{1}, \mathcal{B}$ e $\mathcal{C}$ (parte 3). 


\begin{tabular}{|c|c|c|c|c|c|c|c|}
\hline Arquivo & Alg. $\mathcal{A}_{2}$ & Alg. $\mathcal{A}_{1}$ & Alg. $\mathcal{B}$ & Alg. $\mathcal{C}$ & $l_{\mathcal{C}}$ & $\left(\right.$ Alg. $\left.\mathcal{A}_{2}\right) / l_{\mathcal{C}}$ & {$\left[l_{c}, t+1\right]$} \\
\hline \multicolumn{8}{|c|}{ Ótimo $\leq 300(t=299$ transferências de subárvore $) ; \mathrm{n}=1000$} \\
\hline $001-300$ & 397 & 440 & 583 & 633 & 212 & 1.88 & $212-300$ \\
\hline $002-300$ & 422 & 471 & 595 & 651 & 218 & 1.94 & $218-300$ \\
\hline $003-300$ & 425 & 437 & 566 & 625 & 209 & 2.04 & $209-300$ \\
\hline $004-300$ & 403 & 451 & 584 & 628 & 210 & 1.92 & $210-300$ \\
\hline 005-300 & 417 & 444 & 576 & 621 & 208 & 2.01 & $208-300$ \\
\hline 006-300 & 396 & 435 & 568 & 618 & 207 & 1.92 & $207-300$ \\
\hline 007-300 & 400 & 436 & 589 & 620 & 208 & 1.93 & $208-300$ \\
\hline $008-300$ & 420 & 436 & 574 & 618 & 207 & 2.03 & $207-300$ \\
\hline 009-300 & 413 & 449 & 570 & 623 & 209 & 1.98 & $209-300$ \\
\hline 010-300 & 421 & 454 & 585 & 627 & 210 & 2.01 & $210-300$ \\
\hline \multicolumn{8}{|c|}{ Ótimo $\leq 400(t=399$ transferências de subárvore) $; \mathrm{n}=1000$} \\
\hline $001-400$ & 515 & 556 & 683 & 744 & 249 & 2.07 & $249-400$ \\
\hline $002-400$ & 526 & 562 & 688 & 731 & 245 & 2.15 & $245-400$ \\
\hline $003-400$ & 513 & 553 & 690 & 731 & 245 & 2.10 & $245-400$ \\
\hline $004-400$ & 492 & 546 & 675 & 723 & 242 & 2.04 & $242-400$ \\
\hline 005-400 & 514 & 562 & 680 & 733 & 245 & 2.10 & $245-400$ \\
\hline $006-400$ & 526 & 569 & 676 & 708 & 237 & 2.22 & $237-400$ \\
\hline $007-400$ & 508 & 558 & 688 & 758 & 254 & 2.00 & $254-400$ \\
\hline $008-400$ & 501 & 551 & 673 & 711 & 238 & 2.11 & $238-400$ \\
\hline $009-400$ & 529 & 570 & 673 & 747 & 250 & 2.12 & $250-400$ \\
\hline 010-400 & 539 & 582 & 703 & 751 & 251 & 2.15 & $251-400$ \\
\hline
\end{tabular}

Tabela 5.5: Testes com os Algoritmos $\mathcal{A}_{2}, \mathcal{A}_{1}, \mathcal{B}$ e $\mathcal{C}$ (parte 4 ). 
à qualidade das soluções construídas. Além disso, a razão entre os tamanhos das soluções obtidos pelo Algoritmo $\mathcal{A}_{2}$ e o ótimo é consideravelmente menor do que 3 , aproximando-se de 1,5 à medida que o tamanho da solução ótima se aproxima de 1 .

Todos os programas relatados neste capítulo foram implementados e executados usando-se o sistema operacional Debian GNU/Linux 2.2 em uma máquina Pentium III $866 \mathrm{MHz} 256 \mathrm{Mb}$. O tempo de execução de cada teste neste ambiente foi de poucos segundos.

O código fonte do programa MAF e dos utilitários PRINTTREE, SCRAMBLE, bem como os arquivos gerados por SCRAMBLE para os testes que relatamos neste trabalho, estão disponíveis na página:

http://www.ime.usp.br/〜estela/ime-tese.html 


\section{Capítulo 6}

\section{Algoritmos de aproximação para árvores com grau superior a 2}

Neste capítulo apresentamos uma extensão do Algoritmo $\mathcal{A}_{2}$, que resulta em um algoritmo de $(d+1)$-aproximação para o problema MAF- $d$, onde $d$ é um limitante superior para o grau dos nós das árvores dadas como entrada para este problema. Chamamos esse algoritmo de $\mathcal{D}$.

Para estendermos o Algoritmo $\mathcal{A}_{2}$, precisamos em primeiro lugar estender a operação de eliminação para árvores com grau no máximo $d$. As operações de corte e encolhimento são definidas como no Capítulo 4.

Seja $\mathcal{G}$ uma floresta filogenética com grau no máximo $d$, e um arco de $\mathcal{G}, e_{1}$ um arco de $\mathcal{G}$ tal que $u\left(e_{1}\right)=u(e)$, e $\mathcal{W}$ o componente de $\mathcal{G}$ que contém $e$. A eliminação do arco $e$ de $\mathcal{G}$ é a operação que produz como resultado a floresta filogenética obtida fazendo-se a remoção do arco $e$, resultando na árvore $\mathcal{G}^{*}$, seguida da contração do arco $e_{1}$ em $\mathcal{G}^{*}$ se o nó $u\left(e_{1}\right)$ tiver grau 1 em $\mathcal{G}^{*}$. Veja a Figura 6.1.

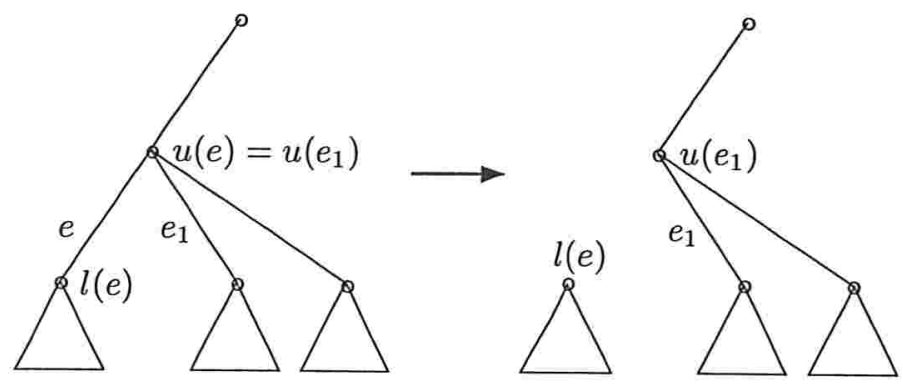

$u(e)$ : grau maior ou igual a 2 .

Figura 6.1: Eliminação em árvores filogenéticas com grau maior ou igual a 2. 
O algoritmo que vamos apresentar para o problema MAF- $d$ é baseado no método básico, com algumas mudanças na definição dos casos, que vamos apresentar a seguir. As definições dadas a seguir estão esquematizadas nas Figuras 6.2, 6.3 e 6.4. A terminologia usada aqui é basicamente a mesma do Capítulo 4.

Em cada iteração do Algoritmo $\mathcal{D}$, procura-se primeiramente um conjunto maximal $P$ com pelo menos dois rótulos tais que as respectivas folhas são irmãs em $\mathcal{G}_{i}$. Vamos chamar esses conjuntos maximais de rótulos de tuplas. Se $\mathcal{G}_{i}$ não admite uma tupla, então o algoritmo devolve 1 mais o número de operações de corte feitas pelo algoritmo e pára.

O passo seguinte é encontrar o subconjunto maximal $A$ de $P$ com pelo menos dois elementos tal que a distância do nó lca $\mathcal{H}_{i}\left(f_{\mathcal{H}_{i}}(A)\right)$ até a raiz do respectivo componente é máxima. Vamos nos referir daqui por diante a este nó como o lca mais distante da raiz em $\mathcal{H}_{i}$. Se todas as folhas de $\mathcal{H}_{i}$ cujos rótulos pertencem a $P$ estão em componentes de $\mathcal{H}_{i}$ distintos, então este lca não existe, e neste caso $A:=P$. Seja $B:=P \backslash A$. Chamamos o conjunto $A$ de a-tupla e o conjunto $B$ de b-tupla. Chamamos de moldura o conjunto de caminhos de cada folha com rótulo na a-tupla até o lca mais distante, e chamamos de hastes da moldura os arcos de $\mathcal{H}_{i}$ que não pertencem aos caminhos da moldura mas cujas extremidades superiores pertencem. Seja $p$ a cardinalidade de $P, q$ a cardinalidade de $B$, e $r$ o número de hastes em $\mathcal{H}_{i}$. Temos, portanto, $p \geq 2, q \geq 0$ e $r \geq 0$.

Definimos cinco casos para o Algoritmo $\mathcal{D}$. Se o lca mais distante não existe, então temos dois casos possíveis, o Caso 3 e o Caso 4. Se o lca mais distante existe, então temos os Casos 1, 2, e 5. As definições de cada caso são:

\begin{tabular}{|ll|}
\hline Caso 1 & $q+r>0$ e $r \leq p$. \\
Caso 2 & $q+r>0$ e $r>p$. \\
Caso 5 & $q+r=0$. \\
\hline Caso 3 & Não há rótulos na tupla com folhas isoladas em $\mathcal{H}_{i}$. \\
Caso 4 & Há pelo menos um rótulo na tupla com a respectiva folha isolada em $\mathcal{H}_{i}$. \\
\hline
\end{tabular}

A Tabela 6.1 lista as transações para o Algoritmo $\mathcal{D}$.

A busca pela tupla em $\mathcal{G}_{i}$ pode ser feita em tempo $O(n)$, sendo $n$ o número de folhas das árvores $\mathcal{T}$ e $\mathcal{U}$ dadas como entrada para o algoritmo, e cada transação pode ser executada em tempo $O(n d)$. Como vimos antes, são executadas $O(n)$ iterações, já que cada eliminação remove ao menos um arco de $\mathcal{T}$ ou de $\mathcal{U}$. A principal diferença entre os Algoritmos $\mathcal{D}$ e $\mathcal{A}_{2}$ está na divisão da tupla entre uma a-tupla e uma b-tupla; essa operação, em conjunto com a contagem das hastes, pode ser implementada em tempo $O\left(n d^{2}\right)$. Portanto o algoritmo $\mathcal{D}$ tem complexidade de tempo $O\left(n^{2} d^{2}\right)$.

A mesma técnica de análise que aplicamos ao Algoritmo $\mathcal{A}_{\bar{k}}$ pode ser utilizada para provar que o Algoritmo $\mathcal{D}$ tem razão de aproximação $d+1$. Em particular, é 
Capítulo 6. Algoritmos de aproximação para árvores com grau superior a 298

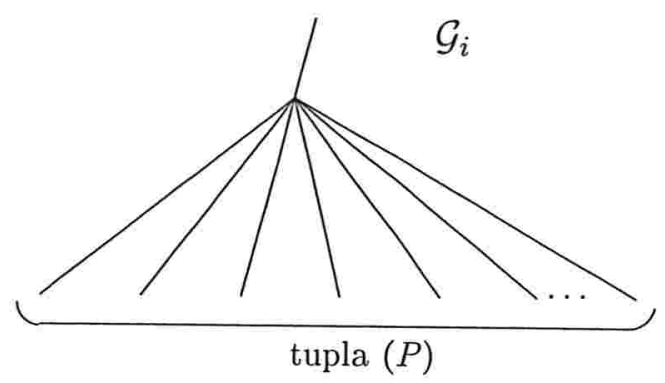

Figura 6.2: Algoritmo $\mathcal{D}$ : tupla em $\mathcal{G}_{i}$.
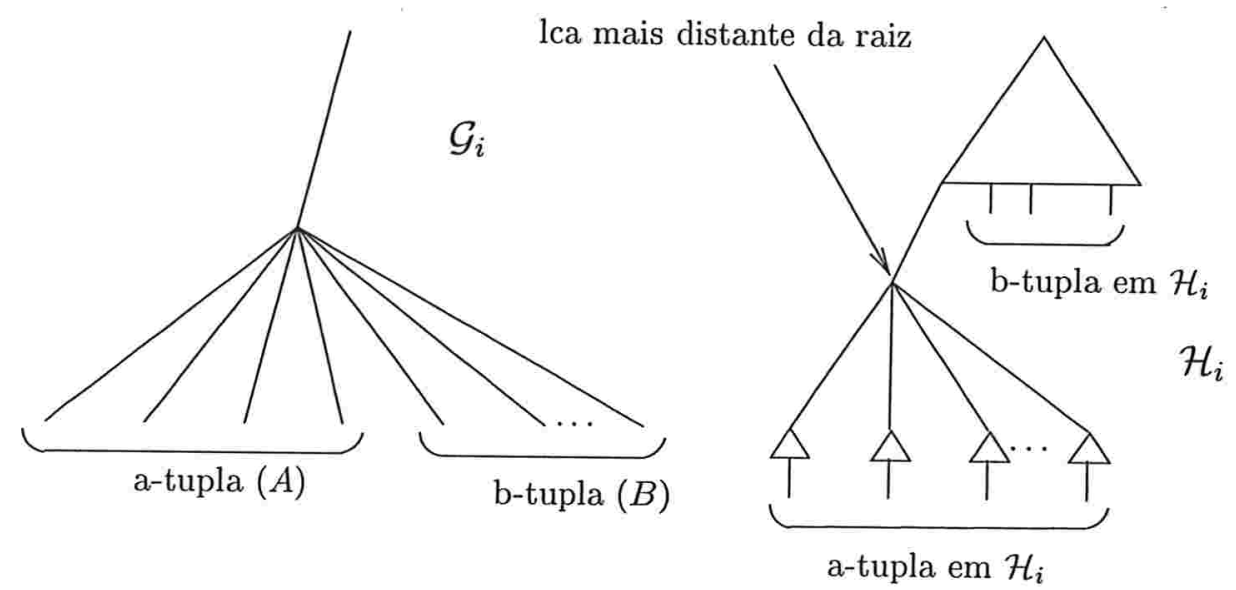

Figura 6.3: Algoritmo $\mathcal{D}$ : a-tupla e b-tupla em $\mathcal{G}_{i}$ e $\mathcal{H}_{i}$.

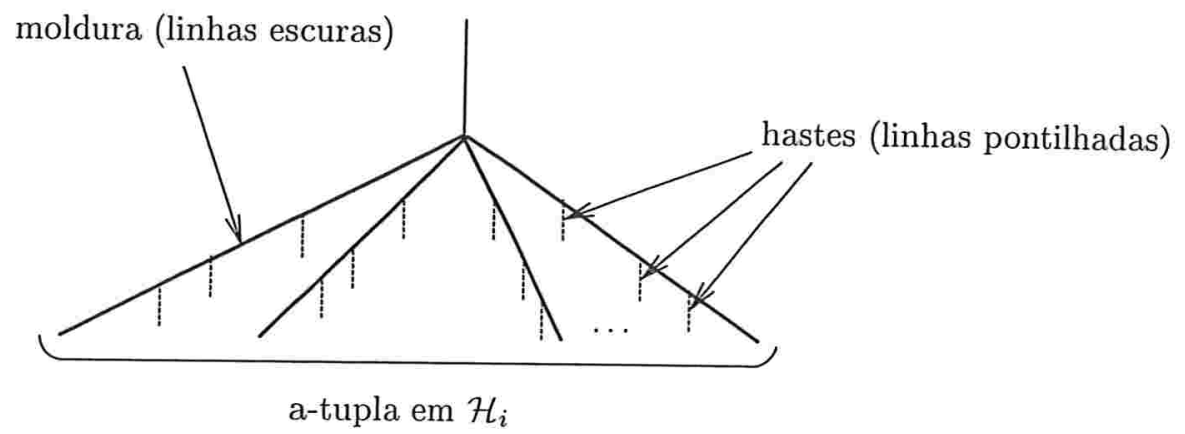

Figura 6.4: Algoritmo $\mathcal{D}$ : moldura e hastes em $\mathcal{H}_{i}$. 
Transações para o Algoritmo $\mathcal{D}$

Caso 1 Cortar em $\mathcal{H}_{i}$ as hastes e as folhas cujos rótulos estão na b-tupla.

Caso 2 Cortar em $\mathcal{G}_{i}$ e $\mathcal{H}_{i}$ as folhas cujos rótulos estão na a-tupla.

Caso 3 Cortar em $\mathcal{H}_{i}$ as folhas cujos rótulos estão na tupla.

Caso 4 Cortar em $\mathcal{G}_{i}$ as folhas cujos rótulos estão na tupla e estão isolados em $\mathcal{H}_{i}$.

Caso 5 Encolher em $\mathcal{G}_{i}$ e $\mathcal{H}_{i}$ as folhas cujos rótulos estão na tupla.

Tabela 6.1: Transações para o Algoritmo $\mathcal{D}$.

válida a seguinte versão do Lema 4.5 :

Lema 6.1 Sejam $\mathcal{G}$ e $\mathcal{H}$ duas florestas filogenéticas com $S_{\mathcal{G}}=S_{\mathcal{H}}$, e seja $\mathcal{H}^{\prime}$ uma restriçãa completa de $\mathcal{H}$ isomorfa a uma restrição completa de $\mathcal{G}$. Suponhamos que $P$ seja uma tupla em $\mathcal{G}$.

1) Se $f_{\mathcal{H}^{\prime}}\left(a_{1}\right)$ e $f_{\mathcal{H}^{\prime}}\left(a_{2}\right)$ com $a_{1}, a_{2} \in A$ estão em componentes de $\mathcal{H}^{\prime}$ distintos e $f_{\mathcal{H}}\left(a_{1}\right)$ e $f_{\mathcal{H}}\left(a_{2}\right)$ não estão isoladas, então pelo menos um dos arcos que incidem em $f_{\mathcal{H}}\left(a_{1}\right)$ e $f_{\mathcal{H}}\left(a_{2}\right)$ é um arco de ligação de $\mathcal{H}$ com respeito a $\mathcal{S}_{\mathcal{H}^{\prime}}$.

2) Se as folhas $f_{\mathcal{H}^{\prime}}(a)$ com $a \in A$ estão em um mesmo componente de $\mathcal{H}^{\prime}$ e a moldura em $\mathcal{H}$ admite ao menos uma haste, então todas as hastes são arcos de ligação com respeito a $S_{\mathcal{H}^{\prime}}$.

Usamos o Lema 6.1 para garantir que, em toda iteração $i$ do Algoritmo $\mathcal{D}$ em que algum arco é cortado mas não ocorre a eliminação de arcos de ligação, existe um conjunto de arcos de ligação ao qual podemos associar as unidades de débito emitidas. Para árvores com grau no máximo $d$, o protocolo de débitos deve ser reformulado conforme explicamos a seguir.

- Se uma iteração do Algoritmo $\mathcal{D}$ é Caso 1 e nenhum arco de ligação é eliminado, então, de acordo com o Lema 6.1, pelo menos um dos arcos incidentes em uma folha cujo rótulo esteja em $A$ é um arco de ligação. Neste caso, as $q+r \leq$ $p+r \leq d$ unidades de débito emitidas nesta iteração são associadas com este arco.

- Se uma iteração do Algoritmo $\mathcal{D}$ é Caso 2 e nenhum arco de ligação é eliminado, então o Lema 6.1 garante que todos os arcos do resíduo das hastes em $\mathcal{H}_{i+1}$ são de ligação. As $p-q \leq d$ unidades de débito emitidas na iteração $i$ são então associadas com este resíduo. 
Capítulo 6. Algoritmos de aproximação para árvores com grau superior a 2100

Resíduos de hastes em $\mathcal{H}_{i}$ produzem barreiras sucessoras à medida que o algoritmo prossegue com as eliminações nas iterações seguintes. Para garantir que, sob as regras do protocolo de débitos, todas as unidades de débito estão quitadas quando o algoritmo pára, a Condição 4.6 precisa ser verificada. O primeiro item desta condição pode ser verificado com um depósito inicial de montante $\alpha(s-1)$ sendo $s$ o tamanho de uma floresta de concordância ótima de $\mathcal{T}$ e $\mathcal{U}$ e $\alpha$ a quantidade de crédito liberada por arco de ligação eliminado. O segundo item deste lema pode ser verificado utilizando-se $d$ quotas de 1 unidade de crédito cada uma. Como o número de cortes feitos por iteração é no máximo $d$, então fazendo $\alpha=d+1$ satisfazemos também este item. 


\section{Capítulo 7}

\section{Resultados de inaproximabilidade}

Hein et al. [20] provam que MAF-2 é NP-difícil e afirmam em seu artigo que esta prova pode ser "facilmente fortalecida para que funcione para MAX SNP-completude", sem contudo apresentarem uma prova de que MAF-2 é MAX SNP-difícil. Neste capítulo apresentamos uma prova de que MAF-2 é APX-difícil. Em nossa prova, a Karpredução da prova de NP-completude de Hein et al. para o MAF-2 é adaptada para que consigamos uma L-redução a partir de um problema ligeiramente diferente daquele utilizado por Hein et al.

Como conseqüências desse resultado temos que os problemas MAF- $d$ para $d \geq 2$ e MAF são APX-difíceis. Mais ainda, como vimos nos capítulos anteriores que existe uma $(d+1)$-aproximação para o problema MAF- $d$, esses resultados mostram que MAF-d é APX-completo.

\subsection{O problema da floresta de concordância ótima com grau no máximo 2 é APX-completo}

A Karp-redução de Hein et al. transforma o problema da cobertura exata por 3conjuntos no problema MAF-2 ${ }_{D}$, a versão decisão do problema MAF (ver Capítulo 2). O problema da cobertura exata por 3-conjuntos, denotado por problema X3C (exact 3-set cover), é definido a seguir:

Dados um conjunto $Z$ com $|Z|=3 q\left(q \in \mathbb{Z}_{*}^{+}\right)$e uma família $\mathcal{C}$ não-vazia de 3 -subconjuntos de $Z$ tal que cada elemento de $Z$ ocorre em no máximo três elementos de $\mathcal{C}$, decidir se $\mathcal{C}$ contém uma cobertura exata de $Z$.

É um fato conhecido que o problema X3C é NP-difícil (ver Garey e Johnson [16]). 
Em nossa adaptação da Karp-redução proposta por Hein et al. vamos utilizar, em lugar de $\mathrm{X} 3 \mathrm{C}$, o problema do empacotamento máximo por 3-conjuntos, que denotamos pela notação M3SP (maximum 3-set packing).

Dados um conjunto finito $Z$ e uma família não-vazia $\mathcal{C}$ de 3 -subconjuntos de $Z$, construir um empacotamento $\mathcal{C}^{\prime} \subseteq \mathcal{C}$ de $Z$ tal que $\mathcal{C}^{\prime}$ tenha cardinalidade máxima.

Berman e Fujito [5] provam que este problema é APX-completo mesmo com a restrição de que cada elemento de $Z$ pertença a no máximo dois conjuntos de $\mathcal{C}$. Vamos nos referir a esta versão do problema como o problema do empacotamento máximo por 3-conjuntos restrito, denotado por M3SP restrito.

Vamos provar a seguir que existe uma L-redução do problema do empacotamento máximo por 3-conjuntos restrito para o problema MAF-2.

Lembramos que uma L-redução de um problema de otimização $\Pi_{1} \in$ NPO para um problema de otimização $\Pi_{2} \in$ NPO consiste em dois algoritmos: um para transformar instâncias de $\Pi_{1}$ em instâncias de $\Pi_{2}$, que chamamos de algoritmo $A$; e o outro, chamado de algoritmo $B$, para transformar instâncias $x$ de $\Pi_{1}$ e soluções viáveis $y$ de instâncias de $\Pi_{2}$ da forma $A(x)$ em soluções viáveis de $\Pi_{1}$. Na L-redução que propomos, o algoritmo $A$ transforma instâncias $(Z, \mathcal{C})$ do problema M3SP restrito em instâncias $A(Z, \mathcal{C}):=(\mathcal{T}, \mathcal{U})$ do problema MAF-2 em que $\mathcal{T}$ e $\mathcal{U}$ seguem a descrição dada a seguir. As árvores $\mathcal{T}$ e $\mathcal{U}$ podem claramente ser construídas em tempo polinomial a partir de $Z$ e $\mathcal{C}$.

Lema 7.1 Existe um algoritmo polinomial que transforma instâncias do problema $M 3 S P$ restrito em instâncias do problema MAF-2.

Prova. Considere uma instância $(Z, \mathcal{C})$ do problema M3SP restrito, constituída por um conjunto $Z:=\left\{z_{1}, \ldots, z_{m}\right\}$ e uma família $\mathcal{C}:=\left\{C_{1}, \ldots, C_{n}\right\}$ de 3 -subconjuntos de $Z$ tais que cada elemento de $Z$ pertence a no máximo dois conjuntos de $\mathcal{C}$.

A instância do problema MAF-2 que vamos construir a partir de $(Z, \mathcal{C})$ é composta de alguns elementos básicos análogos aos elementos do conjunto $Z$ e aos 3-conjuntos pertencentes à família $\mathcal{C}$.

Considere os rótulos $a_{i, j}, b_{i, j}, c_{i, j}$ para $i=1, \ldots, n$ e $j=1, \ldots, m$. Para cada 3conjunto $C_{i}=\left\{z_{i_{1}}, z_{i_{2}}, z_{i_{3}}\right\} \in \mathcal{C}$, sejam $A_{i}$ e $D_{i}$ as árvores filogenéticas da Figura 7.1, sendo que as folhas de $A_{i}$ possuem os rótulos $a_{i, i_{1}}, a_{i, i_{2}}, a_{i, i_{3}}, b_{i, i_{1}}, b_{i, i_{2}}, b_{i, i_{3}}, c_{i, i_{1}}, c_{i, i_{2}}$ e $c_{i, i_{3}}$, e as folhas de $D_{i}$ possuem os rótulos $c_{i, i_{1}}, c_{i, i_{2}}$ e $c_{i, i_{3}}$. Finalmente, para cada elemento $z_{j} \in Z$, seja $B_{j}$ uma árvore filogenética como indicado na Figura 7.1, sendo que as folhas de $B_{j}$ possuem os rótulos $a_{i, j}$ e $b_{i, j}$ para todo $i$ tal que $z_{j} \in C_{i}$ (note que podem existir no máximo dois índices $i$ com esta propriedade; na figura, estes índices estão indicados como $j_{1}$ e $j_{2}$ ). 

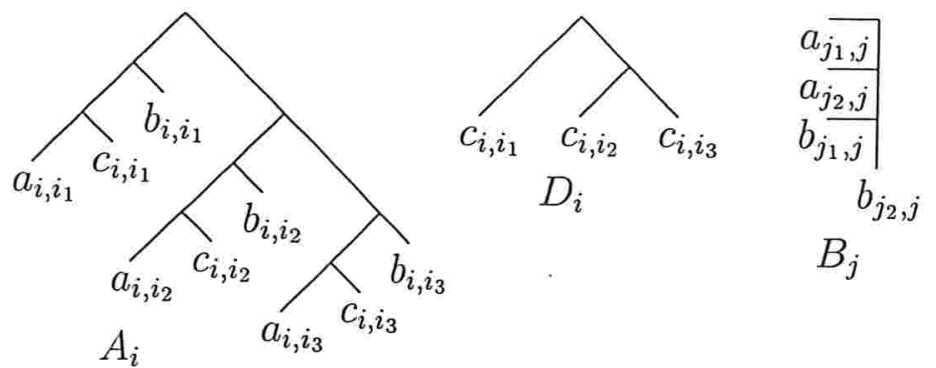

Figura 7.1: Esquemas das árvores $A_{i}, B_{j}$ e $D_{i}$ na L-redução de M3SP para MAF-2 (Lema 7.1).

A árvore filogenética $\mathcal{T}$ da instância desejada consiste de uma cadeia de folhas rotuladas $x_{1}, \ldots, x_{2 n}, y_{1}, \ldots, y_{2 m}$, com cada árvore $A_{i}$ conectada a esta cadeia através de um arco $s\left(r_{A_{i}}\right)$, sendo que as árvores $A_{i}$ aparecem em $\mathcal{T}$ intercaladas a cada duas folhas com rótulos entre $x_{1}$ e $x_{2 n}$. A árvore filogenética $\mathcal{U}$ também consiste em uma cadeia de folhas como em $\mathcal{T}$, porém com as árvores $B_{j}$ conectadas cada uma à cadeia via um arco $s\left(r_{B_{j}}\right)$ e intercaladas a cada duas folhas rotuladas $y_{1}, \ldots$, $y_{2 m}$, e as árvores $D_{i}$ conectadas por arcos $s\left(r_{D_{i}}\right)$ em seqüência no final da cadeia. A Figura 7.2 esquematiza $\mathcal{T}$ e $\mathcal{U}$, e a Figura 7.3 traz um exemplo de instância do problema M3SP restrito com as árvores $\mathcal{T}$ e $\mathcal{U}$ correspondentes. Observe que as árvores $A_{i}, D_{i}$ e $B_{j}$ podem ser vistas também como restrições simples de $\mathcal{T}$ e $\mathcal{U}$; de fato, temos $A_{i}=\mathcal{T}\left|\left\{a_{i, i_{1}}, a_{i, i_{2}}, a_{i, i_{3}}, b_{i, i_{1}}, b_{i, i_{2}}, b_{i, i_{3}}, c_{i, i_{1}}, c_{i, i_{2}}, c_{i, i_{3}}\right\}, B_{j}=\mathcal{U}\right|\left\{a_{i, j}, b_{i, j}\right.$ : $\left.z_{j} \in C_{i}\right\}$ e $D_{i}=\mathcal{U} \mid\left\{c_{i, i_{1}}, c_{i, i_{2}}, c_{i, i_{3}}\right\}$. Claramente esta construção pode ser feita em tempo polinomial.

Um fato importante sobre as árvores $\mathcal{T}$ e $\mathcal{U}$ que acabamos de definir está enunciado no seguinte lema:

Lema 7.2 Se existe uma floresta de concordância $\mathcal{F}$ das árvores $\mathcal{T}$ e $\mathcal{U}$, então existe uma floresta de concordância $\mathcal{F}^{\prime}$ de $\mathcal{T}$ e $\mathcal{U}$ de tamanho $|\mathcal{F}|$ tal que os arcos $s\left(r_{A_{i}}\right)$ para $i=1, \ldots, n$ são arcos de ligação com relação a $\mathcal{F}^{\prime}$.

Prova. Seja $\mathcal{F}$ uma floresta de concordância de $\mathcal{T}$ e $\mathcal{U}$. Suponhamos que $\bar{\imath}$ seja o menor índice entre 1 e $n$ tal que $s\left(r_{A_{\bar{\imath}}}\right)$ não é um arco de ligação com relação a $\mathcal{F}$. Seja $S$ o conjunto em $\mathcal{S}_{\mathcal{F}}$ tal que o componente $\mathcal{T}[S]$ de $T\left[\mathcal{S}_{\mathcal{F}}\right]$ contém o arco $s\left(r_{A_{\bar{\imath}}}\right)$. É fácil verificar que:

(a) Se existe pelo menos um $\hat{\imath}$ tal que $2 \bar{\imath}-1 \leq \hat{\imath} \leq 2 n$ para qual o nó $f_{\mathcal{T}}\left(x_{\hat{\imath}}\right)$ pertence a $\mathcal{T}[S]$, então todas as folhas $f_{\mathcal{T}}\left(x_{2 \bar{\imath}-1}\right), \ldots, f_{\mathcal{T}}\left(x_{2 n}\right)$ exceto $f_{\mathcal{T}}\left(x_{\hat{\imath}}\right)$ estão isoladas em $\mathcal{T}\left[\mathcal{S}_{\mathcal{F}}\right]$. Veja o lado esquerdo da Figura 7.4. 


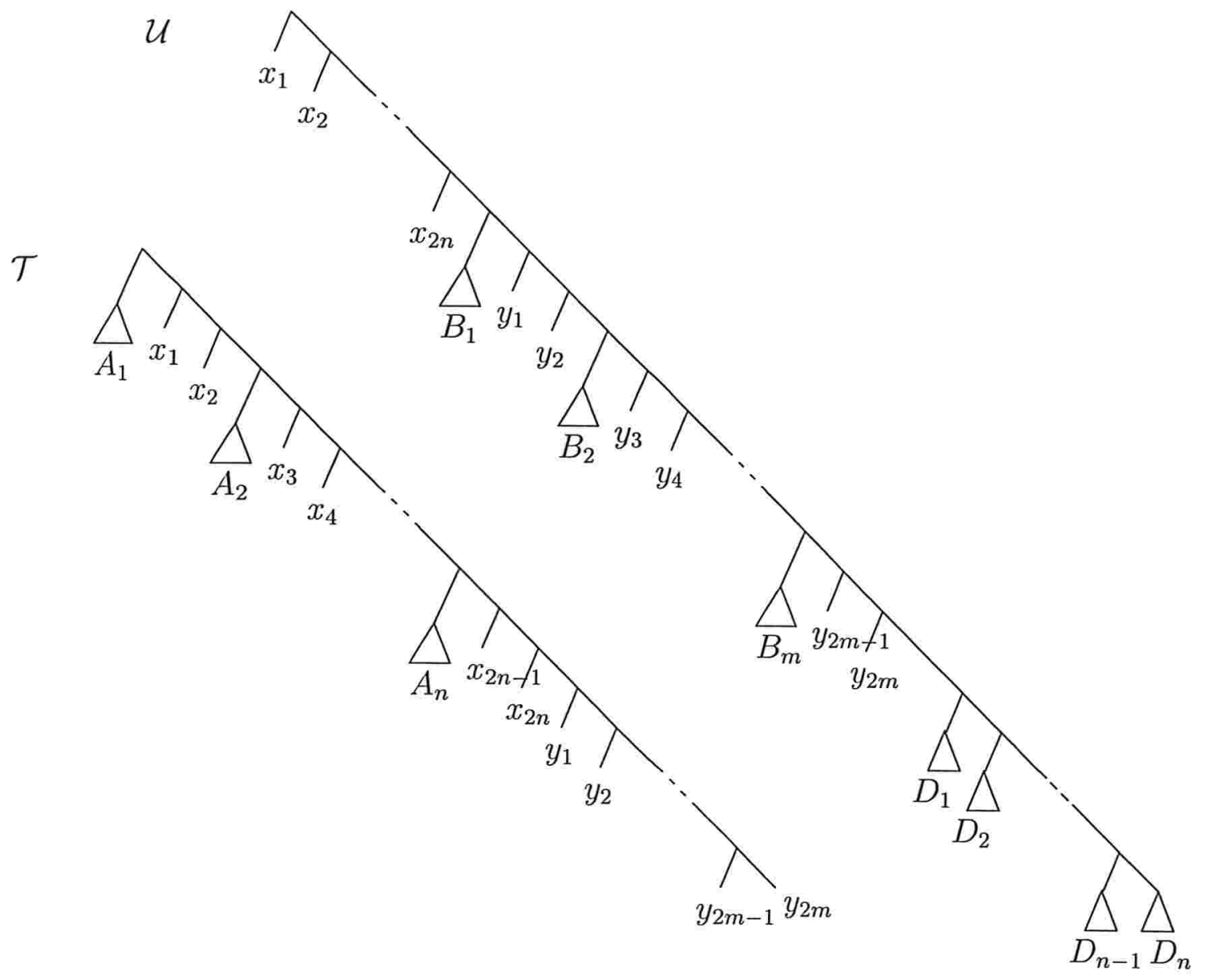

Figura 7.2: Esquemas das árvores $\mathcal{T}$ e $\mathcal{U}$ na L-redução de M3SP para MAF-2 do lema 7.1. 


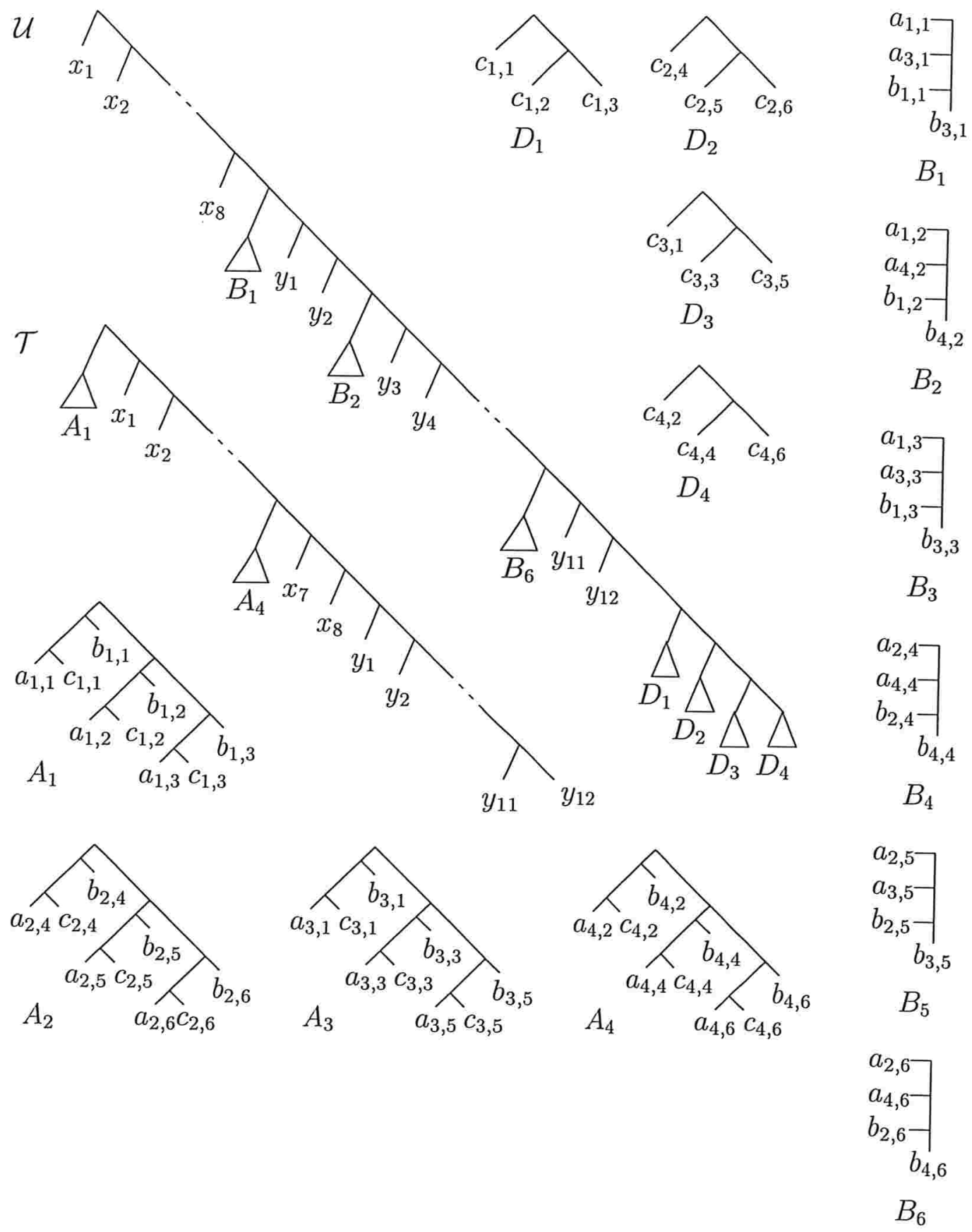

Instância do problema M3SP restrito:

$Z=\left\{z_{1}, z_{2}, z_{2}, z_{4}, z_{5}, z_{6}\right\}$

$\mathcal{C}=\left\{\left\{z_{1}, z_{2}, z_{3}\right\} ;\left\{z_{4}, z_{5}, z_{6}\right\} ;\left\{z_{1}, z_{3}, z_{5}\right\} ;\left\{z_{2}, z_{4}, z_{6}\right\}\right\}$.

Figura 7.3: Um exemplo da construção do Lema 7.1. 


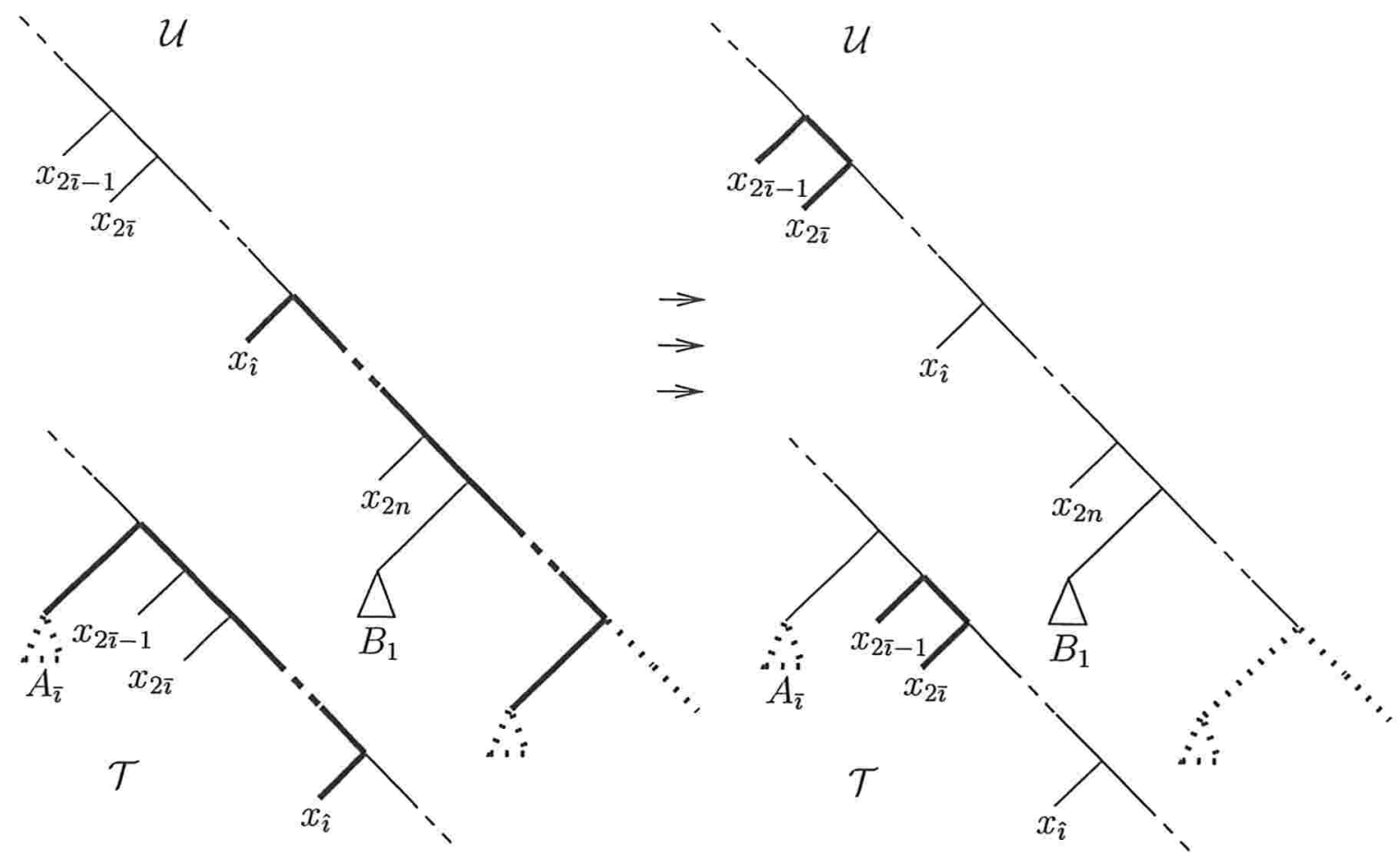

Figura 7.4: Prova do caso (a) do Lema 7.2. Nesta figura e nas duas seguintes os arcos de $\mathcal{T}[S]$ estão em negrito.

(b) Se existe pelo menos um $\hat{\imath}$ tal que $1 \leq \hat{\imath} \leq 2 \bar{\imath}-2$ para o qual o nó $f_{\mathcal{T}}\left(x_{\hat{\imath}}\right)$ pertence a $\mathcal{T}[S]$, então, como no caso acima, todas as folhas $f_{\mathcal{T}}\left(x_{2 \bar{\imath}-1}\right), \ldots, f_{\mathcal{T}}\left(x_{2 n}\right)$, exceto no máximo uma, estão isoladas em $\mathcal{T}\left[\mathcal{S}_{\mathcal{F}}\right]$. Veja o lado esquerdo da Figura 7.5.

(c) Se para $i=1, \ldots, 2 n$ temos que os nós $f_{\mathcal{T}}\left(x_{i}\right)$ pertencem a componentes de $\mathcal{T}\left[\mathcal{S}_{\mathcal{F}}\right]$ diferentes de $\mathcal{T}[S]$, então $f_{\mathcal{T}}\left(x_{2 \bar{\imath}-1}\right)$ e $f_{\mathcal{T}}\left(x_{2 \bar{\imath}}\right)$ estão isoladas em $\mathcal{T}$. Veja o lado esquerdo da Figura 7.6.

Nos três casos acima é possível definir a partir de $\mathcal{F}$ uma floresta de concordância de $\mathcal{T}$ e $\mathcal{U}$ com o mesmo tamanho de $\mathcal{F}$ e tal que $s\left(r_{A_{i}}\right)$ é um arco de ligação de $\mathcal{T}$ com relação a $\mathcal{F}$ para $i=1, \ldots, \bar{\imath}$, como está indicado nos lados direitos das Figuras 7.4, 7.5 e 7.6. Aplicando para cada $\bar{\imath}$ tal que $s\left(r_{A_{i}}\right)$ não é de ligação o procedimento esquematizado nestas figuras, obtemos uma floresta de concordância $\mathcal{F}^{\prime}$ de $\mathcal{T}$ e $\mathcal{U}$ em que todos os $\operatorname{arcos} s\left(r_{A_{i}}\right)$ para $i=1, \ldots, n$ são de ligação.

Pelo mesmo argumento usado na prova do Lema 7.2, podemos obter de $\mathcal{F}^{\prime}$ uma floresta de concordância $\mathcal{F}^{\prime \prime}$ de $\mathcal{T}$ e $\mathcal{U}$ de tamanho $\left|\mathcal{F}^{\prime}\right|$ tal que os $\operatorname{arcos} s\left(r_{A_{i}}\right)$ em $\mathcal{T}$ para $i=1, \ldots, n$ e $s\left(r_{B_{j}}\right)$ em $\mathcal{U}$ para $j=1, \ldots, m$ são arcos de ligação com relação a $\mathcal{F}^{\prime \prime}$. Neste caso, os arcos da forma $s\left(r_{D_{i}}\right)$ em $\mathcal{U}$ para $i=1, \ldots, n$ também são de ligação com relação a $\mathcal{F}^{\prime \prime}$. 


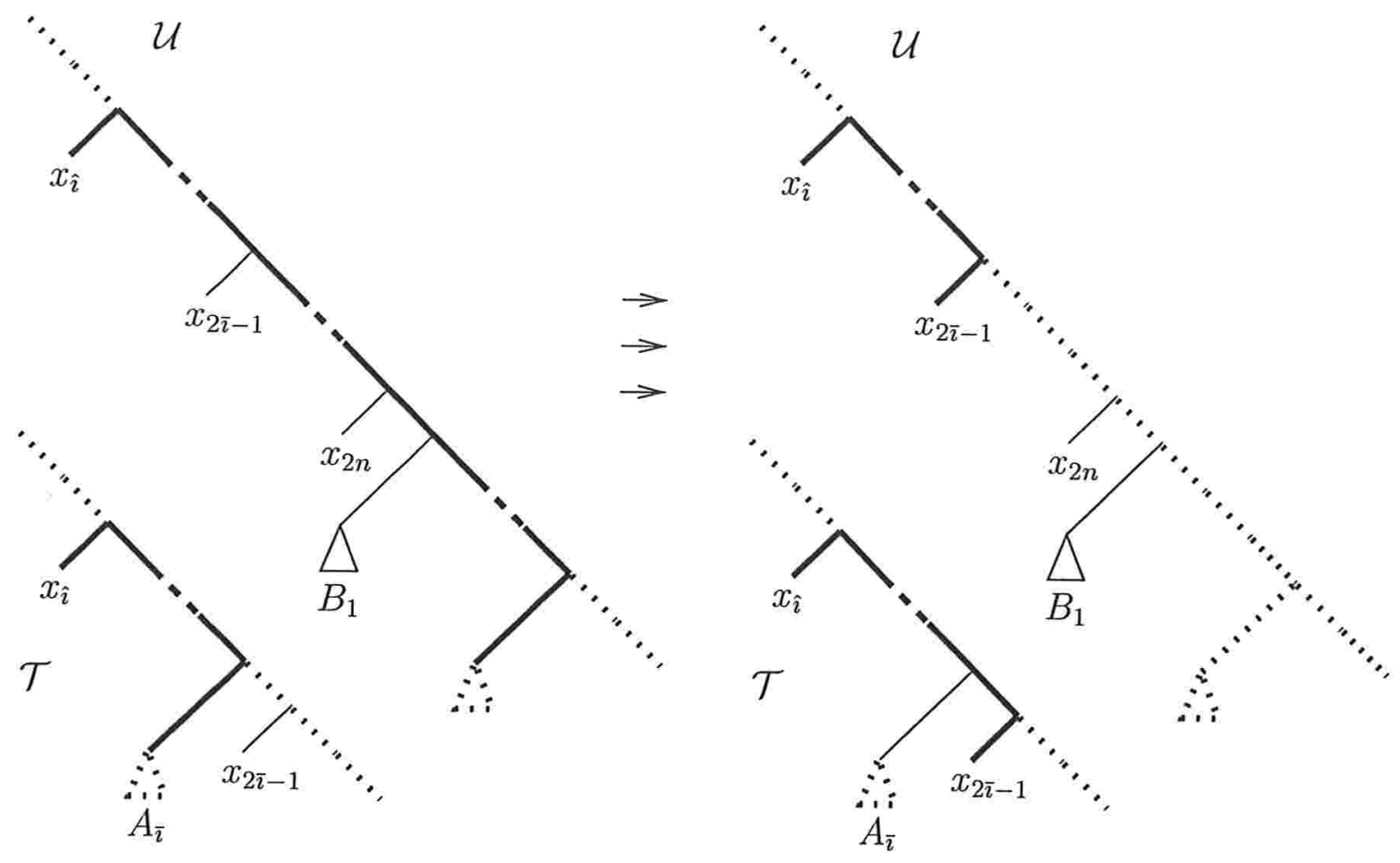

Figura 7.5: Prova do caso (b) do Lema 7.2.
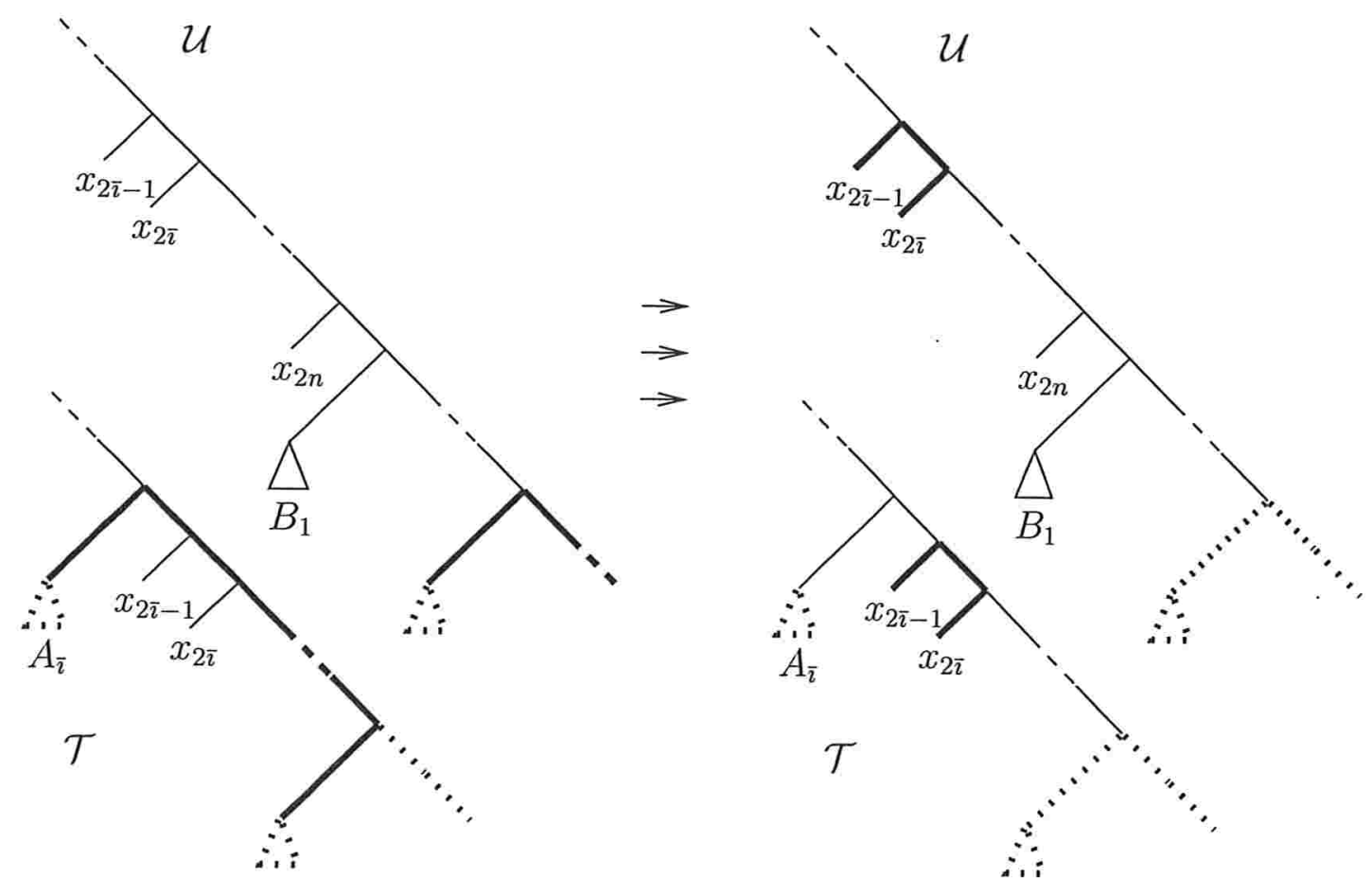

Figura 7.6: Prova do caso (c) do Lema 7.2. 
Para especificarmos o algoritmo $B$ da L-redução, precisamos do lema seguinte:

Lema 7.3 Sejam $\mathcal{T}$ e $\mathcal{U}$ tais que $(\mathcal{T}, \mathcal{U})=A(Z, \mathcal{C})$, onde $A$ é o algoritmo do Lema 7.1 . Se existe uma floresta de concordância de $\mathcal{T}$ e $\mathcal{U}$ com tamanho $7 n+1-p$ para algum $p \geq 0$, então existe um empacotamento $\mathcal{C}^{\prime} \subseteq \mathcal{C}$ de $Z$ de cardinalidade pelo menos $p$.

Prova. Seja $\mathcal{F}$ uma floresta de concordância de $\mathcal{T}$ e $\mathcal{U}$ com tamanho $7 n+1-p$. Pelo Lema 7.2, podemos assumir que os $\operatorname{arcos} s\left(r_{A_{i}}\right)$ de $\mathcal{T}$ e $s\left(r_{D_{i}}\right)$ e $s\left(r_{B_{j}}\right)$ de $\mathcal{U}$ são de ligação com respeito a $\mathcal{S}_{\mathcal{F}}$.

Sejam $A_{i}^{\prime}$ a restrição induzida por $\mathcal{T} \mid \mathcal{S}_{\mathcal{F}}$ sobre $A_{i}, B_{j}^{\prime}$ a restrição induzida por $\mathcal{U} \mid \mathcal{S}_{\mathcal{F}}$ sobre $B_{j}$, e $D_{i}^{\prime}$ a restrição induzida por $\mathcal{U} \mid \mathcal{S}_{\mathcal{F}}$ sobre $D_{i}$. Podemos verificar facilmente que em toda floresta de concordância de $\mathcal{T}$ e $\mathcal{U}$, para $i=1, \ldots, n$ vale:

- Se $f_{\mathcal{T}}\left(c_{i, i_{1}}\right), f_{\mathcal{T}}\left(c_{i, i_{2}}\right)$ e $f_{\mathcal{T}}\left(c_{i, i_{3}}\right)$ não estão todos isolados em $\mathcal{T}\left[\mathcal{S}_{\mathcal{F}}\right]$, então $A_{i}^{\prime}$ possui pelo menos 7 componentes.

- Se $f_{\mathcal{T}}\left(c_{i, i_{1}}\right), f_{\mathcal{T}}\left(c_{i, i_{2}}\right)$ e $f_{\mathcal{T}}\left(c_{i, i_{3}}\right)$ estão isolados em $\mathcal{T}\left[\mathcal{S}_{\mathcal{F}}\right]$, então $A_{i}^{\prime}$ possui pelo menos 6 componentes. Neste caso, se $A_{i}^{\prime}$ possui exatamente 6 componentes, então:

$$
\mathcal{S}_{A_{i}^{\prime}}=\left\{\left\{c_{i, i_{1}}\right\},\left\{c_{i, i_{2}}\right\},\left\{c_{i, i_{3}}\right\},\left\{a_{i, i_{1}}, b_{i, i_{1}}\right\},\left\{a_{i, i_{2}}, b_{i, i_{2}}\right\},\left\{a_{i, i_{3}}, b_{i, i_{3}}\right\}\right\} .
$$

Os 3-conjuntos $C_{i} \in \mathcal{C}$ tais que $A_{i}^{\prime}$ possui exatamente 6 componentes formam portanto um empacotamento de $Z$, já que para cada $z_{j} \in Z, \mathcal{F}$ admite no máximo um componente com as folhas rotuladas $\left\{a_{i, j}, b_{i, j}\right\}$ tal que $z_{j} \in C_{i}$.

Seja $q$ o número de restrições $A_{i}^{\prime}$ que possuem 6 componentes. Temos que $\mathcal{T}\left[\mathcal{S}_{\mathcal{F}}\right]$ possui um componente com as folhas rotuladas $x_{1}, \ldots, x_{2 n}, y_{1}, \ldots, y_{2 m}$, mais $6 q$ componentes derivados das restrições $A_{i}^{\prime}$ que possuem seis componentes, mais pelo menos $7(n-q)$ componentes derivados das demais restrições $A_{i}^{\prime}$. Portanto o tamanho de $\mathcal{F}$ é pelo menos $1+6 q+7(n-q)=7 n+1-q$. Do enunciado do lema temos $7 n+1-p \geq 7 n+1-q$, de onde segue que $q \geq p$.

Como $\mathcal{T}$ e $\mathcal{U}$ sempre admitem (quaisquer que sejam $Z$ e $\mathcal{C}$ ) uma floresta de concordância de tamanho $7 n+1$, então para cada $\mathcal{F} \in \operatorname{SOL}_{\mathrm{MAF}-2}(\mathcal{T}, \mathcal{U})$ temos $m_{\mathrm{MAF}-2}(\mathcal{T}, \mathcal{U}, \mathcal{F})=7 n+1-p$ para algum $p \geq 0$. O Lema 7.3 garante que, neste caso, existe um empacotamento de $Z$ de cardinalidade pelo menos $p$. Vamos, portanto, definir $B(Z, \mathcal{C}, \mathcal{F})$ para $(Z, \mathcal{C}) \in I_{\mathrm{M} 3 S P}$ e $\mathcal{F} \in \mathrm{SOL}_{\mathrm{MAF}-2}(\mathcal{T}, \mathcal{U})$ como sendo este empacotamento. A prova do lema mostra que $B(Z, \mathcal{C}, \mathcal{F})$ pode ser facilmente calculado em tempo polinomial a partir de $Z, \mathcal{C}$ e $\mathcal{F}$. Note que temos, por este mesmo argumento, $m_{\mathrm{MAF}-2}(\mathcal{T}, \mathcal{U}, \mathcal{F}) \geq 7 n+1-m_{\mathrm{M} 3 \mathrm{SP}}((Z, \mathcal{C}), B(Z, \mathcal{C}, \mathcal{F}))$.

Resta provar o seguinte resultado: 
Lema 7.4 Se existe um empacotamento de $Z$ e $\mathcal{C}$ de cardinalidade $p$, então existe uma floresta de concordância de $\mathcal{T}$ e $\mathcal{U}$ com tamanho $7 n+1-p$.

Prova. Seja $\mathcal{C}^{\prime}$ um empacotamento de $Z$ e $\mathcal{C}$ de cardinalidade $p$. Para cada $C_{i} \in \mathcal{C}$, seja:

$$
S_{i}:=\left\{\begin{array}{lll} 
& \left.\left\{c_{i, i_{1}}\right\},\left\{c_{i, i_{2}}\right\},\left\{c_{i, i_{3}}\right\},\left\{a_{i, i_{1}}, b_{i, i_{1}}\right\},\left\{a_{i, i_{2}}, b_{i, i_{2}}\right\},\left\{a_{i, i_{3}}, b_{i, i_{3}}\right\}\right\} & \text { se } C_{i} \in \mathcal{C}^{\prime} \\
\left\{\left\{c_{i, i_{1}}, c_{i, i_{2}}, c_{i, i_{3}}\right\},\left\{a_{i, i_{1}}\right\},\left\{b_{i, i_{1}}\right\},\left\{a_{i, i_{2}}\right\},\left\{b_{i, i_{2}}\right\},\left\{a_{i, i_{3}}\right\},\left\{b_{i, i_{3}}\right\}\right\} & \text { se } C_{i} \notin \mathcal{C}^{\prime} .
\end{array}\right.
$$

Temos que a família $\mathcal{S}:=\left\{\left\{x_{1}, \ldots, x_{2 n}, y_{1}, \ldots, y_{2 m}\right\}\right\} \cup \bigcup_{i=1}^{n} S_{i}$ define uma floresta de concordância de $\mathcal{T}$ e $\mathcal{U}$ com tamanho $1+6 p+7(n-p)=7 n+1-p \square$.

Agora podemos concluir a prova da L-redução que prometemos.

Seja $(Z, \mathcal{C}) \in I_{\mathrm{M} 3 \mathrm{SP}}$. Temos $m_{\mathrm{MAF}-2}^{*}(\mathcal{T}, \mathcal{U}) \leq 7 n$, pois pelo Lema 7.4 vale $m_{\mathrm{MAF}-2}^{*}(\mathcal{T}, \mathcal{U}) \leq 7 n+1-m_{\mathrm{M} 3 \mathrm{SP}}^{*}(Z, \mathcal{C})$, e se $n>0$ então $m_{\mathrm{M} 3 \mathrm{SP}}^{*}(Z, \mathcal{C}) \geq 1$

Devido à restrição feita sobre as instâncias de M3SP de que cada elemento de $Z$ pertence a no máximo dois 3 -conjuntos em $\mathcal{C}$, podemos limitar $n$ por um múltiplo de $m_{\mathrm{M} 3 \mathrm{SP}}^{*}(Z, \mathcal{C})$. Se um empacotamento de $Z$ de cardinalidade máxima tem $p$ 3-conjuntos, então $\mathcal{C}$ possui $p$ 3-conjuntos neste empacotamento máximo, mais no máximo $3 p$ 3-conjuntos, já que cada um dos 3-conjuntos fora do empacotamento máximo intercepta um dos conjuntos neste empacotamento. Portanto, $n \leq 4 m_{\mathrm{M} 3 \mathrm{SP}}^{*}(Z, \mathcal{C})$ e $m_{\mathrm{MAF}-2}^{*}(\mathcal{T}, \mathcal{U}) \leq 28 m_{\mathrm{M} 3 \mathrm{SP}}^{*}(Z, \mathcal{C})$

Finalmente, temos que se $\Delta:=\left|m_{\mathrm{MAF}-2}^{*}(\mathcal{T}, \mathcal{U})-m_{\mathrm{MAF}-2}(\mathcal{T}, \mathcal{U}, \mathcal{F})\right|$, então:

$$
\begin{aligned}
\Delta & =m_{\mathrm{MAF}-2}(\mathcal{T}, \mathcal{U}, \mathcal{F})-m_{\mathrm{MAF}-2}^{*}(\mathcal{T}, \mathcal{U}) \\
& \geq 7 n+1-m_{\mathrm{M} 3 \mathrm{SP}}((Z, \mathcal{C}), B(Z, \mathcal{C}, \mathcal{F}))-m_{\mathrm{MAF}-2}^{*}(\mathcal{T}, \mathcal{U}) \\
& \geq m_{\mathrm{M} 3 \mathrm{SP}}^{*}(Z, \mathcal{C})-m_{\mathrm{M} 3 \mathrm{SP}}((Z, \mathcal{C}), B(Z, \mathcal{C}, \mathcal{F})) \\
& =\left|m_{\mathrm{M} 3 \mathrm{SP}}^{*}(Z, \mathcal{C})-m_{\mathrm{M} 3 \mathrm{SP}}((Z, \mathcal{C}), B(Z, \mathcal{C}, \mathcal{F}))\right|
\end{aligned}
$$

Nesta seção provamos portanto o seguinte resultado:

Teorema 7.5 O problema MAF-2 é APX-difícil.

Uma conseqüência imediata deste resultado é o seguinte teorema:

Teorema 7.6 Os problemas MAF e MAF-d para todo $d \geq 2$ são APX-difíceis.

Como provamos no Capítulo 6 que o problema MAF- $d$ é $(d+1)$-aproximável, então vale o resultado abaixo: 
Teorema 7.7 O problema MAF-d é APX-completo para todo $d \geq 2$.

O Teorema 7.5 justifica o desenvolvimento de algoritmos de aproximação com razão de desempenho constante para resolver MAF-2 e MAF-d, já, que, sob a hipótese de que $\mathrm{P} \neq \mathrm{NP}$, sabemos que não devem existir algoritmos polinomiais, ou mesmo esquemas de aproximação polinomial, que resolvam estes problemas. 


\section{Parte III}

\section{O Problema da Recombinação}




\section{Capítulo 8}

\section{Transferências de subárvore}

\subsection{Transferências canônicas}

A operação geral de transferências de subárvore foi definida no Capítulo 4 como segue:

Seja $\mathcal{W}$ uma árvore filogenética, e $x$ e $y$ dois nós de $\mathcal{W}$ (não necessariamente distintos) tais que $x \neq r_{\mathcal{W}}$ e $y$ não é o antepassado imediato de $x$. A operação de transferência de subárvore em $\mathcal{W}$ definida pelo par de nós $(x, y)$ produz o resultado da seguinte seqüência de operações:

- Eliminação do arco $s_{\mathcal{G}}(x)$, produzindo uma floresta filogenética $\mathcal{W}^{*}$ composta de dois componentes distintos que vamos denominar de $\mathcal{W}_{y}$ e $\mathcal{W}^{\prime}$, sendo $\mathcal{W}_{y}$ o componente de $\mathcal{W}^{*}$ que contém o nó $y$.

- Inserção do componente $\mathcal{W}^{\prime}$ em $\mathcal{W}_{y}$ junto ao nó y.

Esta operação é denotada por $\operatorname{TS}(\mathcal{W}, x, y)$.

Usando a operação geral de transferência de subárvore TS, vamos definir oito subtipos de transferência, cada um dado pelo contexto em que a operação geral pode acontecer. As Figuras 8.1 e 8.2 mostram todos os tipos de transferência de subárvore possíveis. Os arcos eliminados estão assinalados por cruzes e os arcos acrescentados nas operações de inserção são indicados por setas.

É fácil observar que podemos nos concentrar apenas em quatro desses tipos de transferência, sendo que os outros quatro tipos podem ser simulados por estes em apenas um passo. As transferências TS 4 e TS 8 não alteram a árvore dada como argumento, a transferência TS 6 fornece o mesmo resultado que TS 2 e a transferência TS 7 fornece o mesmo resultado que TS 3. Vamos nos concentrar, daqui por diante, apenas nas transferências de tipo TS $1, \operatorname{TS} 2$, TS 3 e TS 5, que serão chamadas transferências canônicas (Figura 8.1). 

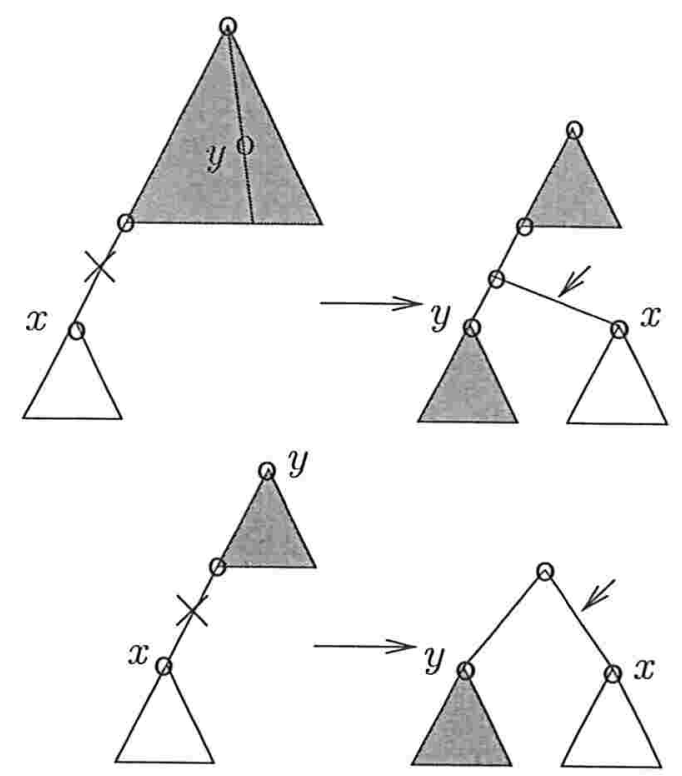

TS 2

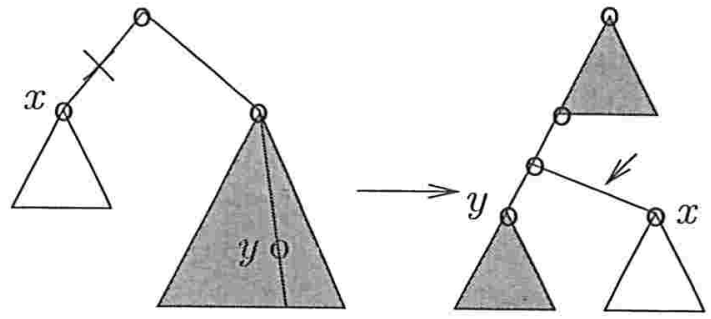

TS 3

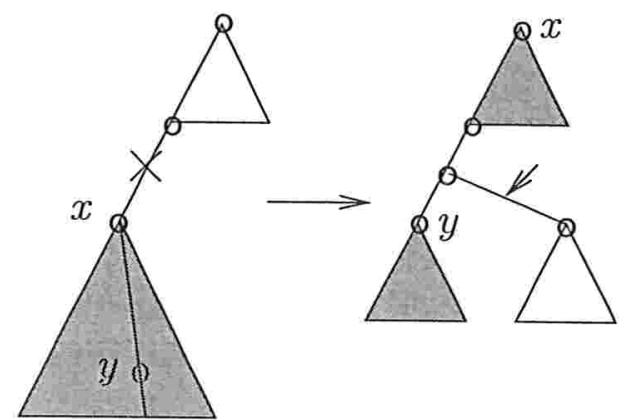

TS 5

Figura 8.1: Tipos possíveis de transferências de subárvore (transferências canônicas). 


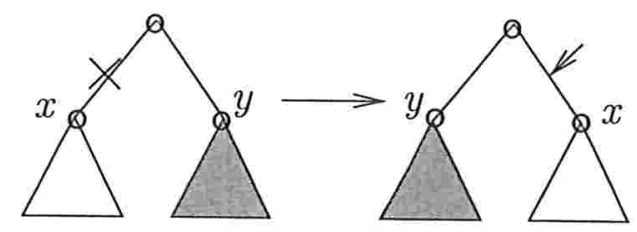

TS 4

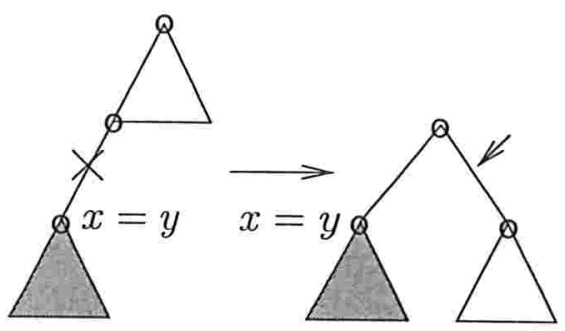

TS 6
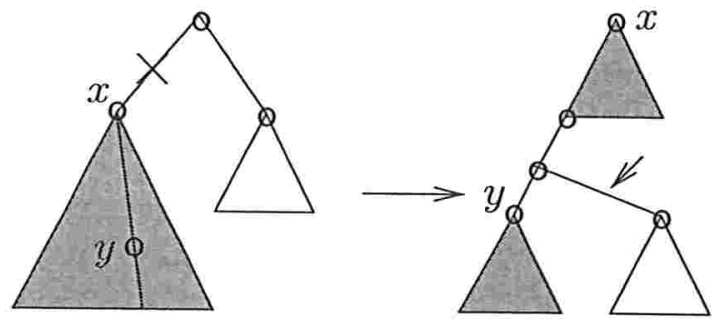

TS 7

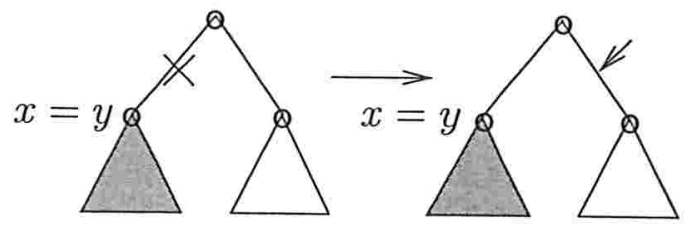

TS 8

Figura 8.2: Tipos possíveis de transferências de subárvore (demais transferências). 
Observe que todos os oito tipos de transferência admitem uma transferência de subárvore inversa, e que a inversa de uma transferência canônica é canônica em todos os quatro casos: a inversa de uma transferência TS 1 é uma outra transferência TS 1, a inversa de uma transferência TS 2 é uma transferência TS 3, a inversa de uma transferência TS 3 é uma transferência TS 2 e a inversa de uma transferência TS 5 é uma outra transferência TS 5.

\subsection{Relação entre seqüências de transferências canônicas e florestas de concordância}

Sejam $\mathcal{T}$ e $\mathcal{U}$ duas árvores filogenéticas. O seguinte resultado estabelece a relação entre uma seqüência de transferências canônicas de comprimento $m$ que transforma $\mathcal{T}$ em $\mathcal{U}$ e uma floresta de concordância de $\mathcal{T}$ e $\mathcal{U}$ de tamanho $m+1$.

Lema 8.1 Sejam $\mathcal{T}$ e $\mathcal{U}$ duas árvores filogenéticas com $S_{\mathcal{T}}=S_{\mathcal{U}}$, e seja $m$ um natural.

- Se existe uma seqüência de transferências de subárvore canônicas de comprimento $m$ que transforma $\mathcal{T}$ em $\mathcal{U}$, então $\mathcal{T}$ e $\mathcal{U}$ admitem uma floresta de concordância com não mais do que $m+1$ componentes.

- Se $\mathcal{T}$ e $\mathcal{U}$ admitem uma floresta de concordância com $m+1$ componentes, então existe uma seqüência de transferências canônicas de comprimento no máximo $m$ que transforma $\mathcal{T}$ em $\mathcal{U}$.

Prova. Vamos provar o primeiro item do resultado acima por indução no comprimento $m$ de uma seqüência de transferências de subárvore que transforma $\mathcal{T}$ em $\mathcal{U}$.

Se $m=0$ então o resultado é imediato.

Se $m \geq 1$, seja $\mathcal{W}$ o resultado da primeira transferência a partir de $\mathcal{T}$. Temos que existe uma seqüência de transferências que transforma $\mathcal{W}$ em $\mathcal{U}$ de comprimento $m-1$, e portanto por hipótese de indução existe uma floresta de concordância $\mathcal{F}$ de $\mathcal{W}$ e $\mathcal{U}$ com não mais do que $m$ componentes. Se o arco $e$ eliminado na transferência que transforma $\mathcal{T}$ em $\mathcal{W}$ é de ligação com respeito a $\mathcal{S}_{\mathcal{F}}$, então $\mathcal{F}$ é uma floresta de concordância de $\mathcal{T}$ e $\mathcal{U}$ com no máximo $m$ componentes. Caso contrário, e pertence a $\mathcal{F}$ e portanto existe um componente de $\mathcal{F}$ que pode ser dividido em dois para compor uma floresta de concordância de $\mathcal{T}$ e $\mathcal{U}$ com não mais do que $m+1$ componentes.

O segundo item também vai ser provado por indução em $m$.

Se $m=0$ então o resultado é imediato. Se $m \geq 1$, seja $\mathcal{F}$ uma floresta de concordância de $\mathcal{T}$ e $\mathcal{U}$ de tamanho $m+1, \mathcal{T}^{\prime}:=\mathcal{T} \mid \overline{\mathcal{S}}_{\mathcal{F}}$ a restrição completa de $\mathcal{T}$ 


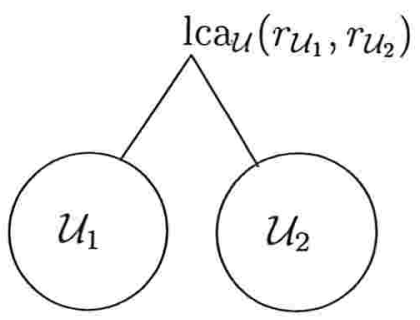

Caso 1

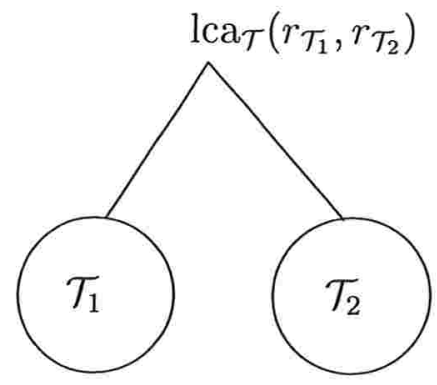

Subcaso 1.1

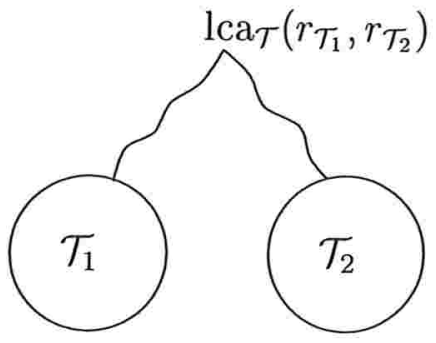

Subcaso 1.2

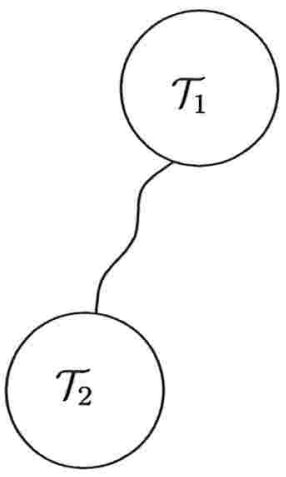

Subcaso 1.3

Figura 8.3: Caso 1 da prova do Lema 8.1.

isomorfa a $\mathcal{F}$, e $\mathcal{U}^{\prime}:=\mathcal{U} \mid \mathcal{S}_{\mathcal{F}}$ a restrição completa de $\mathcal{U}$ isomorfa a $\mathcal{F}$. Vamos dividir a prova em dois casos:

Caso 1: $\mathcal{U}^{\prime}$ possui ao menos um par de componentes $\mathcal{U}_{1}$ e $\mathcal{U}_{2}$ tais que lcau $\left(r_{\mathcal{U}_{1}}, r_{\mathcal{U}_{2}}\right)$ não pertence a nenhum outro componente de $\mathcal{U}^{\prime}$.

Sejam $\mathcal{U}_{1}$ e $\mathcal{U}_{2}$ tais que a distância de $r_{\mathcal{U}_{1}}$ a $r_{\mathcal{U}_{2}}$ é mínima. Então lca ${ }_{\mathcal{U}}\left(r_{\mathcal{U}_{1}}, r_{\mathcal{U}_{2}}\right)$ é o antepassado imediato de $r_{\mathcal{U}_{1}}$ e $r_{\mathcal{U}_{2}}$. Veja a Figura 8.3 (topo).

Sejam $\mathcal{T}_{1}$ e $\mathcal{T}_{2}$ os componentes de $\mathcal{T}^{\prime}$ isomorfos respectivamente a $\mathcal{U}_{1}$ e $\mathcal{U}_{2}$. Podemos ter três subcasos:

Subcaso 1.1: $\operatorname{lca}_{\mathcal{T}}\left(r_{\mathcal{T}_{1}}, r_{\mathcal{T}_{2}}\right) \notin\left\{r_{\mathcal{T}_{1}}, r_{\mathcal{T}_{2}}\right\}$ e $\operatorname{lca}_{\mathcal{T}}\left(r_{\mathcal{T}_{1}}, r_{\mathcal{T}_{2}}\right)$ é o antepassado imediato tanto de $r_{\mathcal{T}_{1}}$ quanto de $r_{\mathcal{T}_{2}}$.

Veja a Figura 8.3 (embaixo, esquerda).

Subcaso 1.2: $\operatorname{lca}_{\mathcal{T}}\left(r_{\mathcal{T}_{1}}, r_{\mathcal{T}_{2}}\right) \notin\left\{r_{\mathcal{T}_{1}}, r_{\mathcal{T}_{2}}\right\}$ e $\operatorname{lca}_{\mathcal{T}}\left(r_{\mathcal{T}_{1}}, r_{\mathcal{T}_{2}}\right)$ não é o antepassado imediato de (sem perda de generalidade) $r_{\mathcal{T}_{2}}$.

Veja a Figura 8.3 (embaixo, centro).

Subcaso 1.3: $\operatorname{lca}_{\mathcal{T}}\left(r_{\mathcal{T}_{1}}, r_{\mathcal{T}_{2}}\right) \in\left\{r_{\mathcal{T}_{1}}, r_{\mathcal{T}_{2}}\right\}$.

Sem perda de generalidade, vamos supor que $\operatorname{lca}_{\mathcal{T}}\left(r_{\mathcal{T}_{1}}, r_{\mathcal{T}_{2}}\right)=r_{\mathcal{T}_{1}}$. Veja a Figura 8.3 (embaixo, direita). 


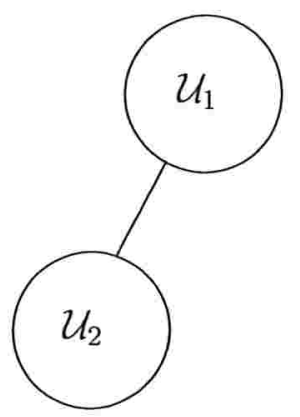

Caso 2

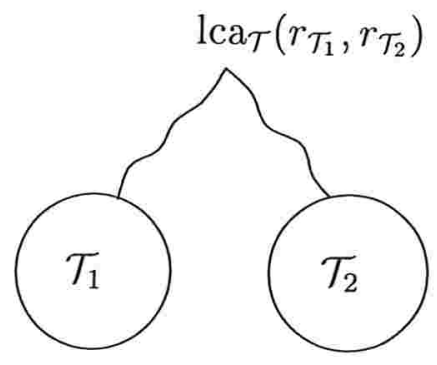

Subcaso 2.1

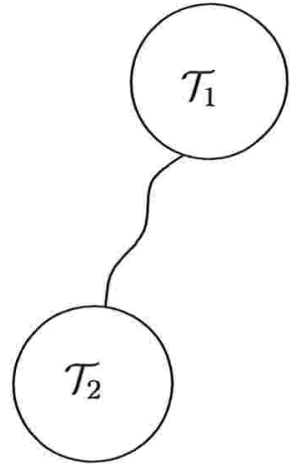

Subcaso 2.2

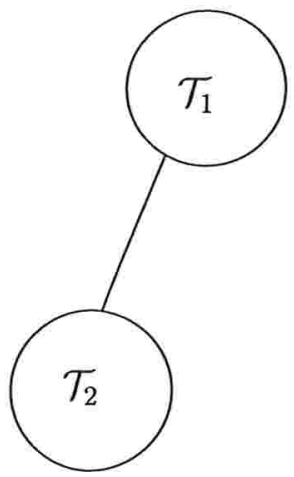

Subcaso 2.3

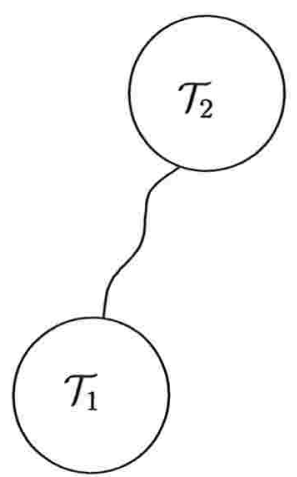

Subcaso 2.4

Figura 8.4: Caso 2 da prova do Lema 8.1.

Nos Subcasos 1.2 e 1.3, usando TS 1 ou TS2, podemos transformar $\mathcal{T}$ em uma árvore filogenética $\mathcal{W}$ tal que $\mathcal{W}$ e $\mathcal{U}$ admitem uma floresta de concordância de tamanho $m$. Pela hipótese de indução, existe uma seqüência com no máximo $m-1$ transferências de subárvore que transforma $\mathcal{W}$ em $\mathcal{U}$. Concatenando a esta seqüência a operação inicial que transforma $\mathcal{T}$ em $\mathcal{W}$, temos uma seqüência de no máximo $m$ transferências que transforma $\mathcal{T}$ em $\mathcal{U}$.

No Subcaso 1.1, os componentes $\mathcal{U}_{1}$ e $\mathcal{U}_{2}$ em $\mathcal{U}^{\prime}$ e $\mathcal{T}_{1}$ e $\mathcal{T}_{2}$ em $\mathcal{T}^{\prime}$ podem ser unidos para formar um par de componentes isomorfos, e portanto podemos obter de $\mathcal{F}$ uma floresta de concordância de $\mathcal{T}$ e $\mathcal{U}$ de tamanho $m$. Pela hipótese de indução, existe uma seqüência com não mais do que $m-1$ transferências de subárvore que transforma $\mathcal{T}$ em $\mathcal{U}$.

Caso 2: Para todo par $\mathcal{U}_{1}, \mathcal{U}_{2}$ de componentes de $\mathcal{U}^{\prime}$, temos que lcau $\left(r_{\mathcal{U}_{1}}, r_{\mathcal{U}_{2}}\right)$ pertence a algum componente de $\mathcal{U}^{\prime}$.

Sem perda de generalidade, vamos supor que $r_{\mathcal{U}_{2}}$ pertence a $\mathcal{U}_{1}$. Veja a Figura 8.4 (topo). Podemos ter quatro subcasos, que listamos a seguir. Nesses casos, considere que $\mathcal{T}_{1}$ e $\mathcal{T}_{2}$ são definidos como no Caso 1 . 
Subcaso 2.1: Existe um par de componentes $\mathcal{U}_{1}, \mathcal{U}_{2}$ para o qual lca ${ }_{\mathcal{T}}\left(r_{\mathcal{T}_{1}}, r_{\mathcal{T}_{2}}\right) \notin$ $\left\{r_{\mathcal{T}_{1}}, r_{\mathcal{T}_{2}}\right\}$.

Veja a Figura 8.4 (embaixo, esquerda).

Subcaso 2.2: Existe um par de componentes $\mathcal{U}_{1}, \mathcal{U}_{2}$ de $\mathcal{U}^{\prime}$ para o qual lca $\left(r_{\mathcal{T}_{1}}, r_{\mathcal{T}_{2}}\right)=r_{\mathcal{T}_{1}}$ e $r_{\mathcal{T}_{2}}$ não pertence a $\mathcal{T}_{1}$.

Veja a Figura 8.4 (embaixo, centro-esquerda).

Subcaso 2.3: Existe um par de componentes $\mathcal{U}_{1}, \mathcal{U}_{2}$ de $\mathcal{U}^{\prime}$ para o qual lca $\left(r_{\mathcal{T}_{1}}, r_{\mathcal{T}_{2}}\right)=r_{\mathcal{T}_{1}}$ e $r_{\mathcal{T}_{2}}$ pertence a $\mathcal{T}_{1}$.

Veja a Figura 8.4 (embaixo, centro-direita).

Subcaso 2.4: $\operatorname{lca}_{\mathcal{T}}\left(r_{\mathcal{T}_{1}}, r_{\mathcal{T}_{2}}\right)=r_{\mathcal{T}_{2}}$ para todo par $\mathcal{U}_{1}, \mathcal{U}_{2}$ de $\mathcal{U}^{\prime}$ que satisfazem o Caso 2.

Veja a Figura 8.4 (embaixo, direita).

Nos Subcasos 2.1 e 2.2 é possível transformar $\mathcal{T}$ em uma árvore filogenética $\mathcal{W}$ tal que $\mathcal{W}$ e $\mathcal{U}$ admitem uma floresta de concordância de tamanho $m$ aplicando TS 1 ou TS 3. Pelo mesmo argumento usado nos Subcasos 1.2 e 1.3, obtemos uma seqüência de transferências de subárvore com no máximo $m$ operações que transforma $\mathcal{T}$ em $\mathcal{U}$. O Subcaso 2.3 é similar ao Subcaso 1.1.

Já no Subcaso 2.4 temos uma situação diferente. Neste subcaso temos necessariamente todos os componentes de $\mathcal{T}^{\prime}$ com as raízes aparecendo em um único caminho folha-raiz, e todas as raízes dos componentes de $\mathcal{U}^{\prime}$ em caminhos folharaiz em que as raízes aparecem na ordem inversa em que elas aparecem em $\mathcal{T}$. A Figura 8.5 exemplifica essa situação.

Neste caso, sejam $\mathcal{T}_{2}$ o componente de $\mathcal{T}^{\prime}$ tal que $r_{\mathcal{T}_{2}}=r_{\mathcal{T}}$ (componente $4_{\mathcal{T}}$ na Figura 8.5), $\mathcal{U}_{2}$ o componente de $\mathcal{U}^{\prime}$ correspondente a $\mathcal{T}_{2}$ (componente $\mathcal{U}_{\mathcal{U}}$ na Figura 8.5), $\mathcal{U}_{1}$ o componente de $\mathcal{U}^{\prime}$ tal que $r_{\mathcal{U}_{2}}$ pertence a $\mathcal{U}_{1}$ (componente $3 \mathcal{U}$ na Figura 8.5) e $\mathcal{T}_{1}$ o componente de $\mathcal{T}^{\prime}$ correspondente a $\mathcal{U}_{1}$ (componente $3_{\mathcal{T}}$ ). Aplicando TS 5, obtemos de $\mathcal{T}$ uma árvore filogenética $\mathcal{W}$ tal que $\mathcal{W}$ e $\mathcal{U}$ admitem uma floresta de concordância de tamanho $m$; a Figura 8.6 mostra o resultado da aplicação de TS 5 ao exemplo da Figura 8.5. Observe que $\mathcal{W}$ também satisfaz o Subcaso 2.4. Portanto aplicando o procedimento para este caso $m$ vezes a partir de $\mathcal{T}$ e $\mathcal{U}$, obtemos uma seqüência de $m$ transferências de subárvore que transforma $\mathcal{T}$ em $\mathcal{U}$

O resultado acima tem uma conseqüência importante: ele permite estabelecer a equivalência entre uma determinada noção de distância entre árvores filogenéticas baseada em transferências de subárvore, e a distância que é dada por florestas de concordância. Vamos definir em primeiro lugar a noção de distância baseada em transferências.

Se $\mathcal{T}$ e $\mathcal{U}$ são duas árvores filogenéticas tais que $S_{\mathcal{T}}=S_{\mathcal{U}}$, então denotamos por $\operatorname{SPR}$-canônica $(\mathcal{T}, \mathcal{U})$ o número mínimo de operações de transferência de subárvore 


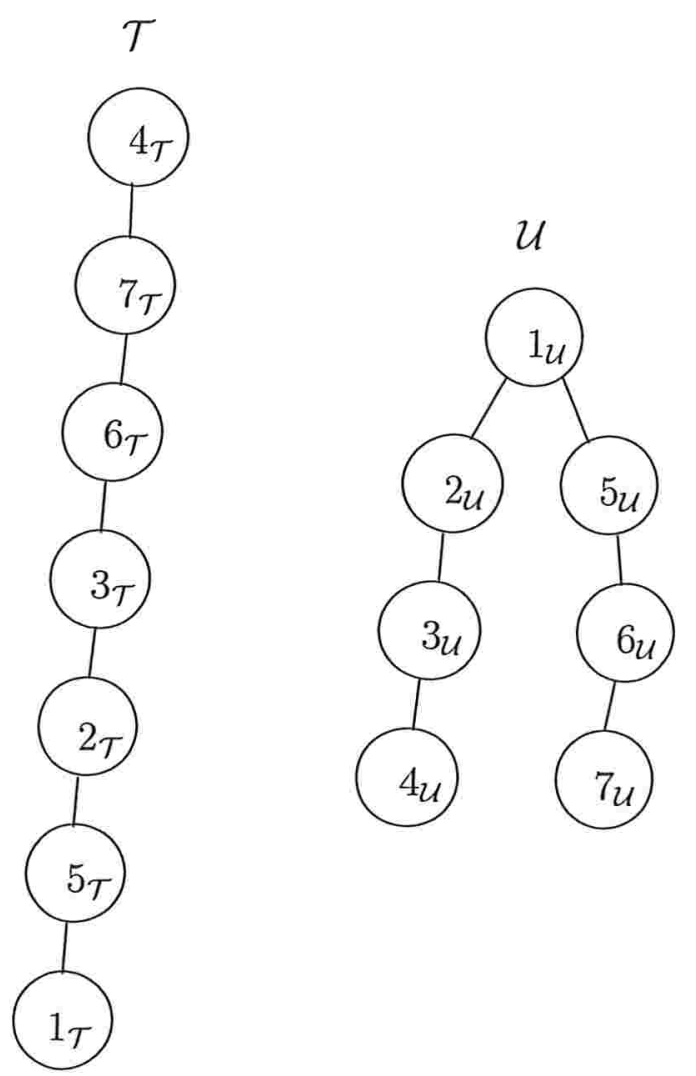

Figura 8.5: Exemplo para o Subcaso 2.4 da prova do Lema 8.1. 


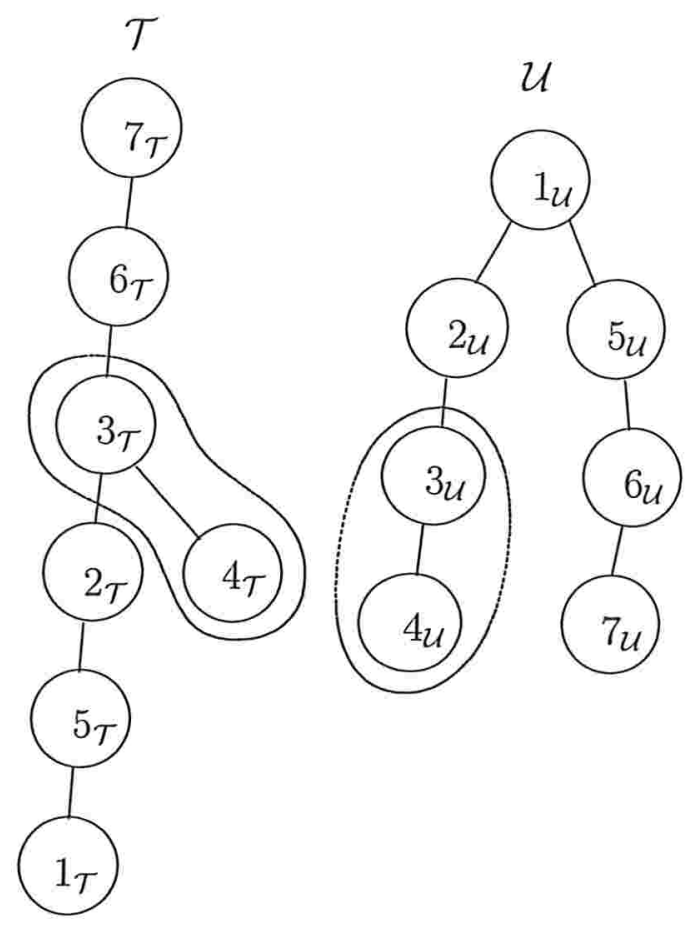

Figura 8.6: Resultado da aplicação de TS 5 ao exemplo da Figura 8.5.

que transforma $\mathcal{T}$ em $\mathcal{U}$, e por $\operatorname{MAF}(\mathcal{T}, \mathcal{U})$ o tamanho de uma floresta de concordância ótima de $\mathcal{T}$ e $\mathcal{U}$.

O seguinte resultado, que estabelece a equivalência entre $\operatorname{MAF}(\mathcal{T}, \mathcal{U})$ e $\operatorname{SPR}$-canônica $(\mathcal{T}, \mathcal{U})$, é uma conseqüência da propriedade anterior:

Teorema 8.2 Sejam $\mathcal{T}$ e $\mathcal{U}$ árvores filogenéticas com $S_{\mathcal{T}}=S_{\mathcal{U}}$. Então

$$
\operatorname{SPR} \text {-canônica }(\mathcal{T}, \mathcal{U})=\operatorname{MAF}(\mathcal{T}, \mathcal{U})-1
$$

Prova. Suponhamos que $m:=\operatorname{SPR}$-canônica $(\mathcal{T}, \mathcal{U})$. Então, pela primeira parte do Lema 8.1 temos que $\operatorname{MAF}(\mathcal{T}, \mathcal{U}) \leq m+1$. Pela segunda parte podemos concluir que $\operatorname{MAF}(\mathcal{T}, \mathcal{U})=m+1$. De fato, $\operatorname{se} \operatorname{MAF}(\mathcal{T}, \mathcal{U})$ fosse menor do que $m+1$ então existiria uma seqüência de transferências de subárvore de comprimento menor que $m$ que transforma $\mathcal{T}$ em $\mathcal{U}$, contrariando o fato de que $\operatorname{SPR}$-canônica $(\mathcal{T}, \mathcal{U})=m$. 


\subsection{Conversão de florestas de concordância em seqüên- cias de transferências de subárvore}

A prova do Lema 8.1 fornece um algoritmo que, dada uma floresta de concordância $\mathcal{F}$ de $\mathcal{T}$ e $\mathcal{U}$, calcula uma seqüência de transferências de subárvore canônicas que transforma $\mathcal{T}$ em $\mathcal{U}$ com $|\mathcal{F}|-1$ operações. Este algoritmo, que vamos chamar de Algoritmo TransfCan, é especificado a seguir. Comentários no algoritmo são colocados entre chaves.

Algoritmo TransfCan

Entrada: Duas árvores filogenéticas $\mathcal{T}$ e $\mathcal{U}$ tais que $S_{\mathcal{T}}=S_{\mathcal{U}}$ e uma floresta de concordância $\mathcal{F}$ de $\mathcal{T}$ e $\mathcal{U}$;

Saída: Seqüência de transferências de subárvore com no máximo $|\mathcal{F}|-1$ operações canônicas que transforma $\mathcal{T}$ em $\mathcal{U}$.

(1) $\mathcal{T}^{\prime}:=\mathcal{T} \mid \mathcal{S}_{\mathcal{F}}$;

(2) $\mathcal{U}^{\prime}:=\mathcal{U} \mid \mathcal{S}_{\mathcal{F}}$;

(3) Enquanto $\mathcal{U}^{\prime}$ possui mais do que um componente

(4) Se $\mathcal{U}^{\prime}$ possui um par de componentes $\mathcal{U}_{1}, \mathcal{U}_{2}$ tal que lca ${ }_{\mathcal{U}}\left(r_{\mathcal{U}_{1}}, r_{\mathcal{U}_{2}}\right)$ não pertence a nenhum componente de $\mathcal{U}^{\prime}$ então $\{$ Caso 1$\}$

(5) Sejam $\mathcal{U}_{1}$ e $\mathcal{U}_{2}$ dois tais componentes tais que $r_{\mathcal{U}_{1}}$ e $r_{\mathcal{U}_{2}}$ têm distância mínima;

(6) Sejam $\mathcal{T}_{1}$ e $\mathcal{T}_{2}$ os componentes de $\mathcal{T}^{\prime}$ isomorfos respectivamente a $\mathcal{U}_{1}$ e $\mathcal{U}_{2}$;

(7) $\quad \operatorname{Se~} \operatorname{lca} \mathcal{T}_{\mathcal{T}}\left(r_{\mathcal{T}_{1}}, r_{\mathcal{T}_{2}}\right) \notin\left\{r_{\mathcal{T}_{1}}, r_{\mathcal{T}_{2}}\right\}$ então

senão

Se a distância entre $r_{\mathcal{T}_{1}}$ e $r_{\mathcal{T}_{2}}$ é maior do que 2 então $\{$ Subcaso 1.2\} $\operatorname{TS}\left(\mathcal{T}, r_{\mathcal{T}_{2}}, r_{\mathcal{T}_{1}}\right)$; $\{$ senão Subcaso 1.1\}

senão $\{$ Subcaso 1.3$\}$

$$
\begin{array}{r}
\text { Se lca } \operatorname{lca}_{\mathcal{T}}\left(r_{\mathcal{T}_{1}}, r_{\mathcal{T}_{2}}\right)=r_{\mathcal{T}_{1}} \\
\text { então } \operatorname{TS}\left(\mathcal{T}, r_{\mathcal{T}_{2}}, r_{\mathcal{T}_{1}}\right) ; \\
\text { senão } \operatorname{TS}\left(\mathcal{T}, r_{\mathcal{T}_{1}}, r_{\mathcal{T}_{2}}\right) ;
\end{array}
$$

Se existem $\mathcal{U}_{1}$ e $\mathcal{U}_{2}$ tais que $r_{\mathcal{U}_{2}}$ pertence a $\mathcal{U}_{1}$ e lca $\operatorname{lc}_{\mathcal{T}}\left(r_{\mathcal{T}_{1}}, r_{\mathcal{T}_{2}}\right) \neq r_{\mathcal{T}_{2}}$; então

Se $r_{\mathcal{T}_{2}}$ não pertence a $\mathcal{T}_{1}$ então $\{$ Subcasos 2.1 e 2.2$\}$

Seja $v$ o nó de $\mathcal{T}_{1}$ que corresponde ao nó irmão de $r_{\mathcal{U}_{2}}$ em $\mathcal{U}_{1}$; $\operatorname{TS}\left(\mathcal{T}, r_{\mathcal{T}_{2}}, v\right)$ \{senão Subcaso 2.3$\}$ senão $\{$ Subcaso 2.4 \} 
(24) Imprima todas as transferências de subárvore realizadas.

A atualização no passo 21 deste algoritmo consiste em obtermos a partir de $\mathcal{F}$ uma floresta de concordância das árvores $\mathcal{T}$ e $\mathcal{U}$ como elas se apresentam antes das atualizações nas linhas 22 e 23. Se $\mathcal{F}_{1}$ e $\mathcal{F}_{2}$ são os componentes de $\mathcal{F}$ isomorfos a $\mathcal{T}_{1}$ e $\mathcal{T}_{2}$ no início de uma dada iteração, então a atualização une $\mathcal{F}_{1}$ e $\mathcal{F}_{2}$ em um novo componente e com isso obtém uma nova floresta de concordância de $\mathcal{T}$ e $\mathcal{U}$ no passo 21. O fato de que o tamanho de $\mathcal{F}$ diminui em uma unidade a cada iteração garante a parada do algoritmo e também que o comprimento da seqüência de operações obtida construída é no máximo $|\mathcal{F}|-1$. 


\section{Capítulo 9}

\section{Modelagem de eventos de recombinação}

Definições de conceitos gerais de Biologia Computacional e Sistemática presentes neste capítulo podem ser encontradas respectivamente em Swofford et al. [33] e Setubal e Meidanis [32].

Dados sequenciais podem estar sujeitos a fenômenos que violam a suposição do modelo evolutivo tradicional de que a informação filogenética se difunde entre os táxons seguindo uma topologia em árvore. O mais importante desses fenômenos é a recombinação, ou seja, a produção de seqüências descendentes pelo crossing-over de duas seqüências antepassadas imediatas. Essas novas seqüências possuem, em geral, trechos intercalados herdados de cada uma de suas seqüências antepassadas imediatas. Os limites entre esses trechos são chamados de pontos de recombinação.

Considere três cadeias de caracteres $s, t, u$ sobre um alfabeto $\Sigma$. Se existem um inteiro $c>0$ e seqüências

$$
\bar{s}=\left(s_{1}, s_{2}, \ldots, s_{c+1}\right), \bar{t}=\left(t_{1}, t_{2}, \ldots, t_{c+1}\right), \bar{u}=\left(u_{1}, u_{2}, \ldots, u_{c+1}\right)
$$

tais que $s, t, u$ podem ser escritas respectivamente como

$$
s_{1}, s_{2}, \ldots, s_{c+1} ; t_{1}, t_{2}, \ldots, t_{c+1} ; u_{1}, u_{2}, \ldots, u_{c+1}
$$

tal que $u_{i}$ é obtido de $s_{i}$ para $i$ ímpar e de $t_{i}$ para $i$ par através de uma série de mutações pontuais (ou seja, inserção, deleção e substituição de caracteres), e temos $\left|s_{i}\right|>0$ para $i$ ímpar, $\left|t_{i}\right|>0$ para $i$ par e $\left|u_{i}\right|>0$ para todo $i$, então $(c, \bar{s}, \bar{t}, \bar{u})$ é uma recombinação entre $s$ e $t$ com resultado $u$. O inteiro $c$ indica o número de pontos de recombinação.

Em um contexto que envolve fenômenos de recombinação a hipótese da topologia em árvore claramente não se sustenta. Já que toda cadeia que é produto de um evento 
de recombinação herda informação genética de duas fontes, devem aparecer circuitos na topologia da história evolutiva.

Portanto, é necessário discutir as topologias a serem empregadas para representar a evolução de grupos de seqüências sujeitas a recombinação. Uma proposta nesse sentido foi apresentada por Ma, Wang e Li [29]. Nessa proposta, as seguintes condições devem ser satisfeitas para que um grafo orientado acíclico represente tais processos evolutivos:

- haja pelo menos um nó designado como raiz;

- nenhum arco saia de qualquer das raízes;

- não haja circuitos orientados no grafo;

- se $v$ for um nó distinto de qualquer das raízes, então existe pelo menos um e não mais do que dois arcos que saem de $v$.

Vamos nos referir a tais topologias como topologias com recombinação, ou redes (networks). Os nós dos quais saem dois arcos são ditos nós de recombinação, e tais arcos são chamados de arcos de recombinação. Observamos que topologias do tipo acima, quando não apresentam nós de recombinação, não apresentam também circuitos, e portanto correspondem às árvores enraizadas definidas no Capítulo 1.

\subsection{O modelo de Hein para recombinações}

O modelo de Hein para representação de seqüências sobreviventes de DNA préalinhadas relacionadas por um mesmo processo evolutivo e sujeitas a recombinação é descrito em Hein [18]. No modelo evolutivo tradicional, em que não consideramos a ocorrência de eventos de recombinação, supomos que uma única árvore filogenética modela a relação evolutiva das seqüências ao longo do alinhamento. Hein argumenta que, na presença de recombinação, para cada coluna do alinhamento podemos ter uma árvore filogenética diferente, e que árvores filogenéticas de colunas vizinhas estão relacionadas por operações de transferência de subárvore, sendo que cada evento de recombinação que acontece entre duas colunas vizinhas no alinhamento corresponde a uma transferência de subárvore entre as árvores filogenéticas correspondentes.

O esquema na Figura 9.1 mostra o modelo de Hein com seqüências de DNA presentes nos nós. Na parte superior temos a rede que representa a evolução das seqüências $7,10,11$ e 12 . A notação $(5,6)$ para a seqüência presente no nó de recombinação mostrado neste esquema indica que esta seqüência é resultado do crossingover de duas outras seqüências, 5 e 6 , sendo que 5 é descendente da seqüência 4 e 6 é 

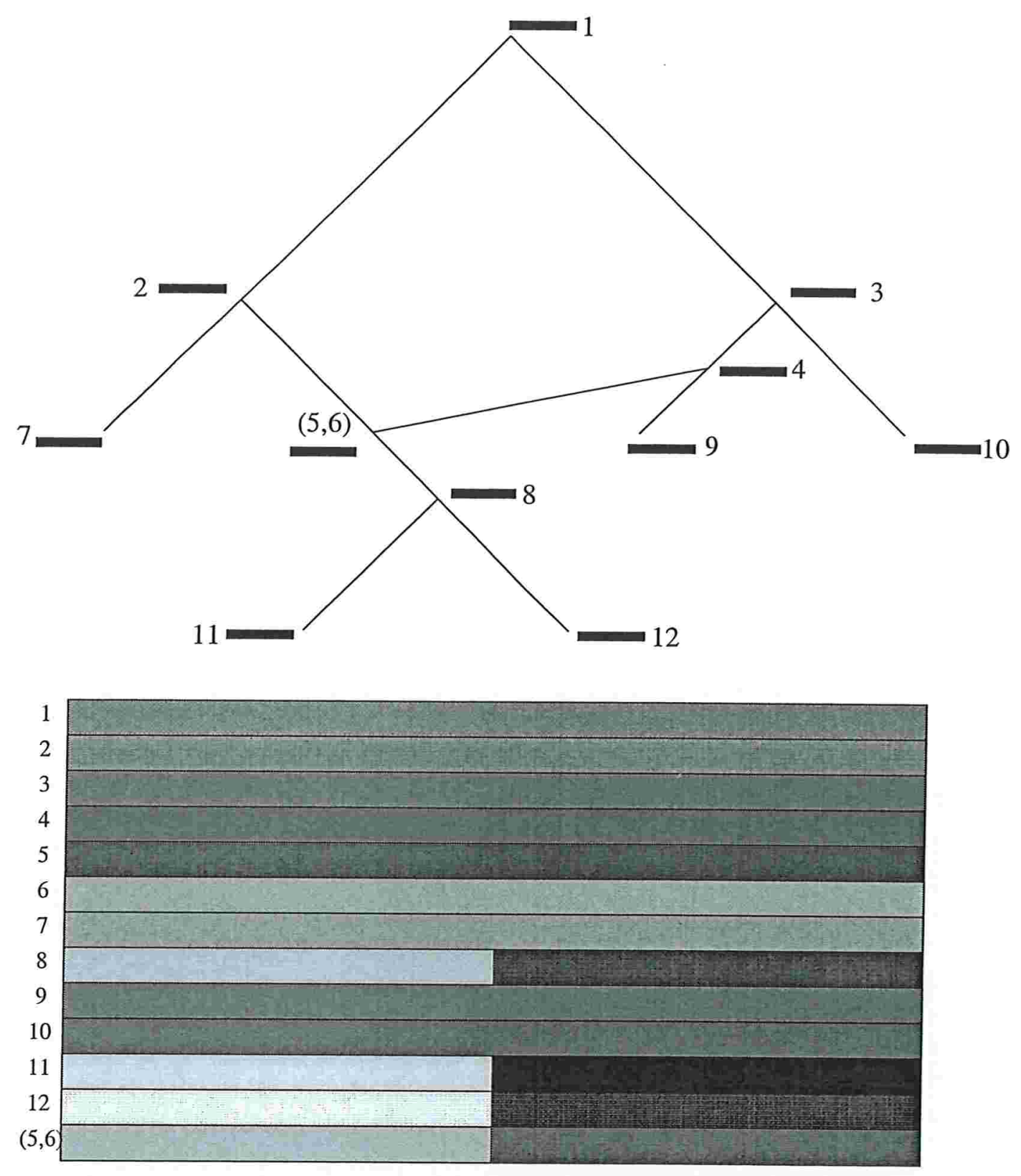

Figura 9.1: O modelo de Hein. 
descendente da seqüência 2. A parte inferior da figura mostra o alinhamento múltiplo das seqüências sobreviventes e ancestrais, inclusive as seqüências 5 e 6 .

As Figuras 9.2 e 9.3 mostram as árvores filogenéticas derivadas da rede mostrada na Figura 9.1. A árvore filogenética derivada esquerda corresponde à metade esquerda do alinhamento mostrado na Figura 9.1 e a árvore filogenética derivada direita corresponde à metade direita desse alinhamento. As figuras mostram ainda os respectivos alinhamentos. Observe que a seqüência $(5,6)$ não aparece em nenhuma das árvores derivadas, pois para isso precisaríamos ter nós de grau 1 nessas árvores. Pelo mesmo motivo a seqüência 4 não aparece na árvore derivada esquerda e a seqüência 2 não aparece na árvore derivada direita.

Podemos observar facilmente que, nas Figuras 9.2 e 9.3, a árvore derivada direita pode ser obtida da árvore derivada esquerda pela transferência de subárvore que movimenta os nós com as seqüências 8, 11 e 12 . Note ainda que não precisamos necessariamente considerar transferências de subárvore sendo feitas apenas no sentido da árvore derivada esquerda para a árvore derivada direita: podemos usar também o sentido inverso, da árvore direita para a árvore esquerda.

As próximas seções tratam de estudar a relação precisa entre eventos de recombinação e operações de transferência de subárvore. Isso envolve discutir quais tipos de operações de transferência são adequadas para modelar eventos de recombinação e quais não o são.

\subsection{Transferências equivalentes a eventos de recombina- ção}

As características das transferências canônicas de subárvore fazem com que elas pareçam a princípio adequadas para modelar eventos de recombinação. Vimos que toda transferência de subárvore pode ser simulada em um passo por uma transferência canônica e que toda transferência canônica admite uma operação inversa que é uma transferência canônica. A outra propriedade que mencionamos como adequada para transferências de subárvore é a equivalência entre eventos de recombinação e transferências canônicas. Essa segunda propriedade vai ser verificada em seguida para cada um dos tipos de transferências canônicas.

Seja $\mathcal{W}$ uma árvore filogenética, e $\mathcal{W}^{\prime}$ o resultado de uma transferência canônica $\operatorname{TS}(\mathcal{W}, x, y)$ sobre $\mathcal{W}\left(x \neq r_{\mathcal{W}}, y \neq\right.$ antepassado imediato de $x$ em $\left.\mathcal{W}\right)$. Precisamos verificar se a transferência que produz $\mathcal{W}^{\prime}$ equivale a um evento de recombinação nos casos em que:

(a) $\operatorname{lca}(x, y)=y$; 


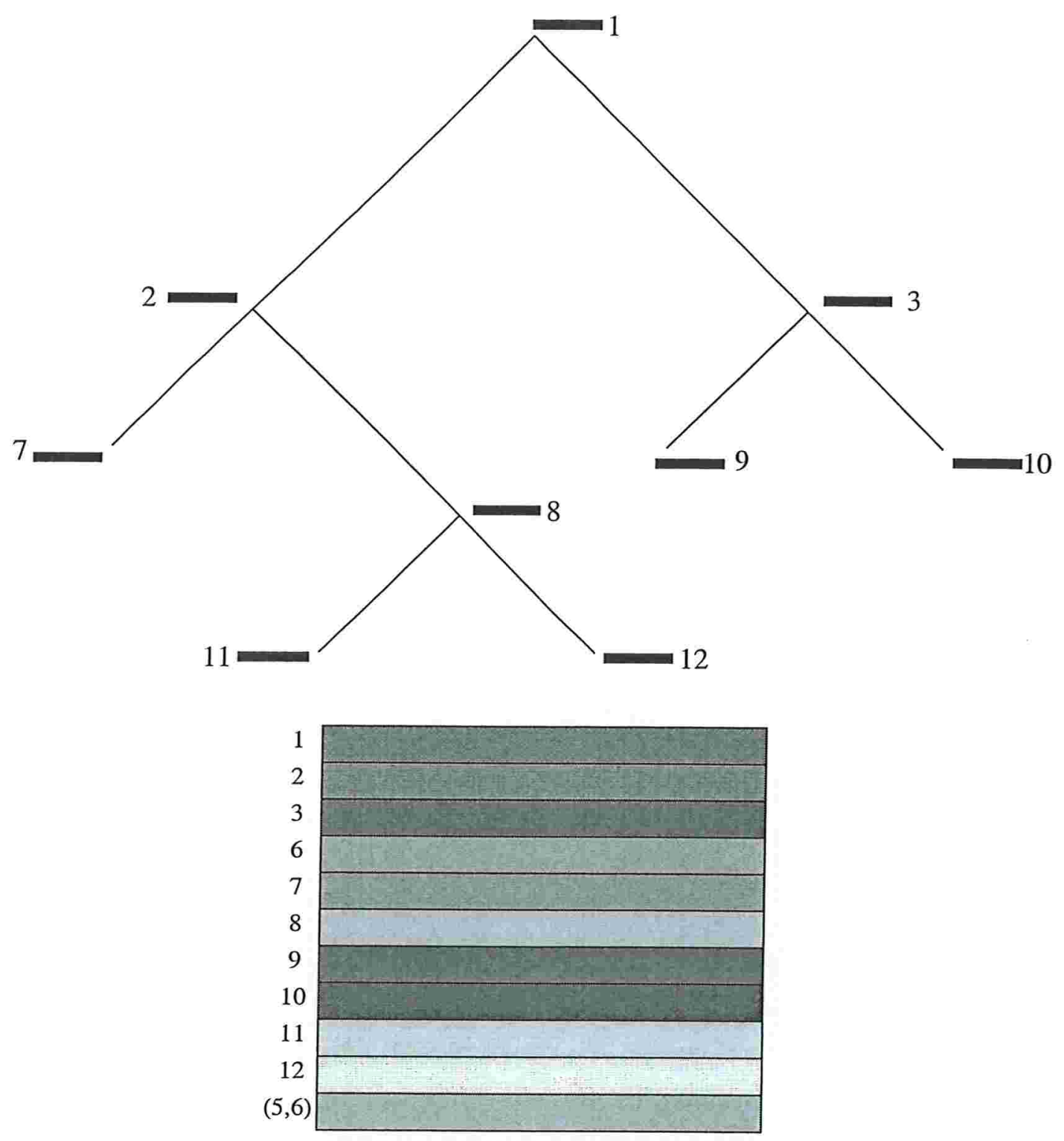

Figura 9.2: Árvore derivada esquerda e alinhamento correspondente. 


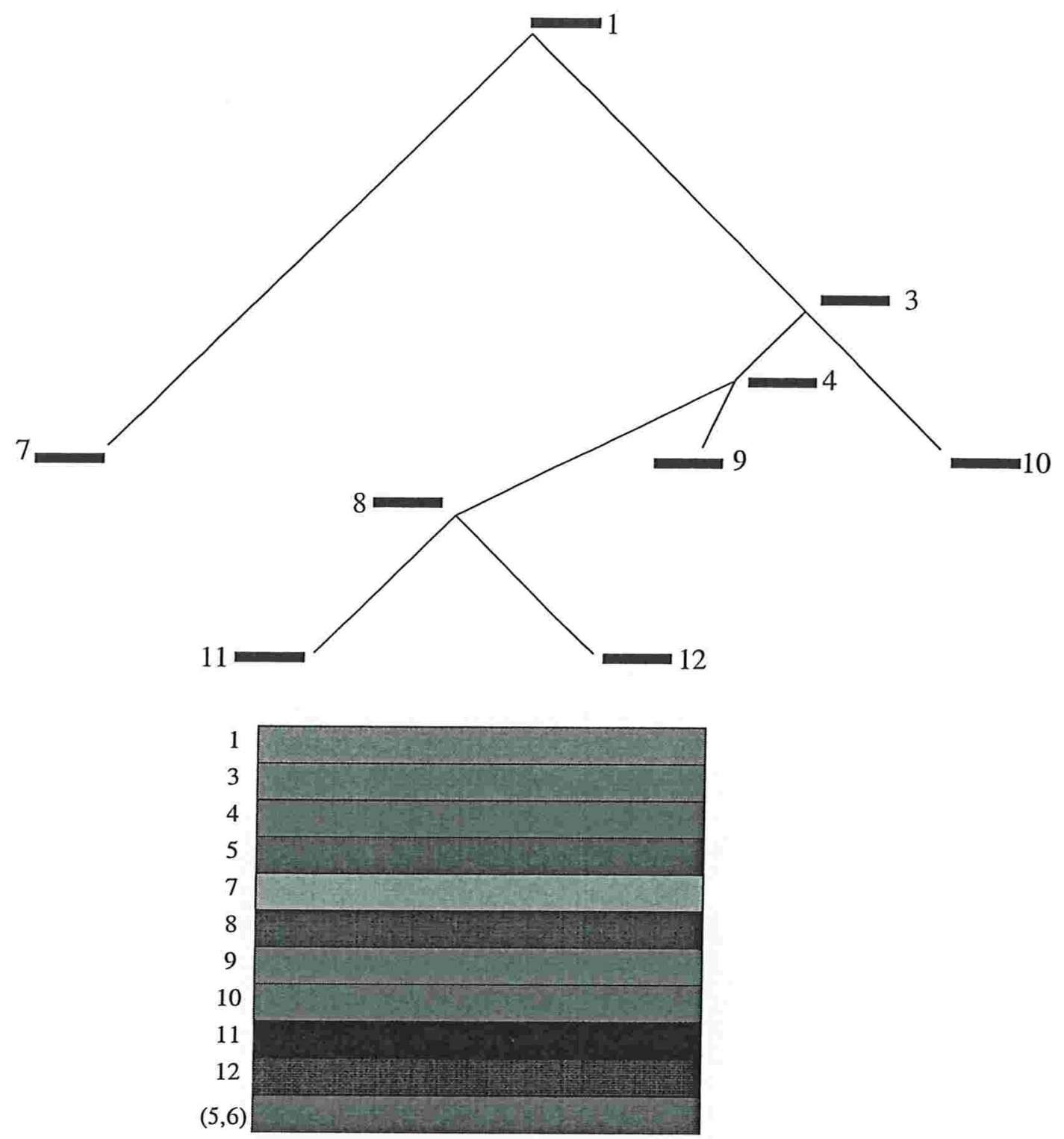

Figura 9.3: Árvore derivada direita e alinhamento correspondente. 

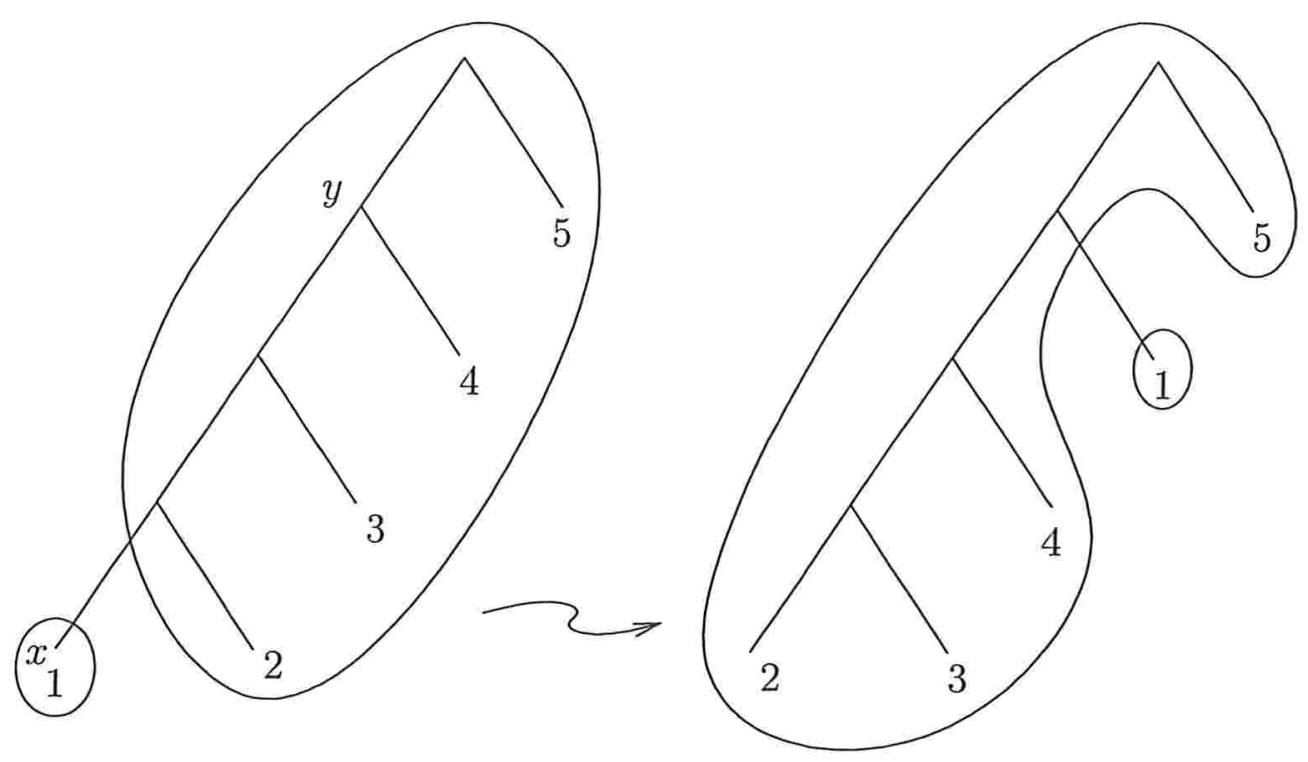

Figura 9.4: Exemplo em que uma transferência de subárvore faz com que um componente seja reconectado em um nó antepassado na árvore resultante.

(b) $\operatorname{lca}(x, y)=x$;

(c) lca $(x, y) \notin\{x, y\}$ e $y$ descende do irmão de $x$;

(d) $\operatorname{lca}(x, y) \notin\{x, y\}$ e $y$ não descende do irmão de $x$.

Os casos (a) e (b) são discutidos a seguir. O caso (d) foi exemplificado na Seção 9.1. O caso (c) é o inverso do caso (a), e sua validade pode ser garantida pelos mesmos argumentos que serão utilizados nesse caso.

Essa divisão em casos tem por objetivo simplificar a discussão que vai se seguir, já que ela é mais adequada para esse propósito do que a divisão dada pelas transferências canônicas. A equivalência desses casos com as transferências canônicas é a seguinte: TS 5 é o caso (b), TS 3 é um subcaso do caso (c) (em que lca $(x, y)=r_{\mathcal{W}}$ ), TS 2 é um subcaso do caso (a) (em que $y=r_{\mathcal{W}}$ ) e TS 1 é o caso (d) mais os subcasos de (c) e (a) restantes.

\subsubsection{Recombinações que ligam uma restrição em um nó antepassado}

Aqui estamos nos referindo ao caso (a). A Figura 9.4 mostra um exemplo.

Esse movimento é perfeitamente legal, conforme a Figura 9.5 mostra. Na parte superior da figura, mostramos códigos para as seqüências nos nós ancestrais em ambas 
as árvores filogenéticas, e na parte inferior mostramos o que seria a rede da qual essas árvores derivam. O exemplo mostra que, na verdade, na árvore derivada esquerda o nó com a seqüência 1 descende do nó que contém a seqüência 6 , enquanto que na árvore derivada direita o nó com a seqüência 1 descende do nó com a seqüência 10. Observe que pode ser necessário supor a existência de um antepassado para a seqüência que está no nó raiz de $\mathcal{W}$ nos casos em que $y=r_{\mathcal{W}}$, do mesmo modo como supomos um novo antepassado (seqüência 10) para a seqüência 8 na Figura 9.5. (a).

O exemplo acima mostra que não aparecem circuitos orientados nas redes no caso

\subsubsection{Recombinações que geram circuitos orientados em redes}

Uma situação mais difícil de analisar ocorre quando temos duas árvores derivadas (que podem ser adjacentes ou não, no sentido de que uma pode ou não ser obtida da outra através de uma única transferência canônica de subárvore) nas quais, considerandose todas as seqüências que aparecem nas duas árvores, acontece de termos ao menos um par de seqüências A e B com A sendo antepassada de B numa árvore e B sendo antepassada de A na outra. Ou seja, a rede resultante apresenta um circuito orientado. Essa é a situação do caso (b). Um exemplo disso aparece na Figura 9.6.

Nesta figura, temos as sequiências 3 e 6 aparecendo nas duas árvores derivadas, que distam uma da outra apenas por uma transferência de subárvore de tipo TS 5. Se consideramos que a ordem entre os lcas dos componentes da floresta de concordância induzida pela transferência de subárvore precisa ser preservada nas duas árvores derivadas (pois essa é a ordem em que as seqüências dos lcas surgem ao longo do tempo), então a situação mostrada nesse exemplo é inaceitável, pois temos 3 descendendo de 6 em uma região do alinhamento e 6 descendendo de 3 em outra região. Mas, mesmo se desconsideramos a condição de que a ordem dos lcas seja preservada, temos ainda um outro motivo para não aceitar essa situação.

A Figura 9.7 mostra o que seriam uma rede e um alinhamento múltiplo correspondentes a esta situação. As letras e números dentro de cada sequiência no alinhamento indicam a seqüência antepassada imediata. Na rede vemos uma coisa que é mencionada em vários artigos sobre topologia com suporte para recombinação: a ocorrência de mais de uma raiz (ver por exemplo Hein [18]; Ma, Wang e Li [29]; e Kececioglu e Gusfield [24]). As duas raízes estão indicadas como R1 e R2, e estamos supondo que existe uma seqüência artificial correspondendo a cada uma delas. $\mathrm{O}$ alinhamento esclarece o que está acontecendo. Nele vemos a seqüência 3 do lado esquerdo do alinhamento descendendo da seqüência $\mathrm{A}$ (ou mais precisamente, de um descendente de A), e do lado direito descendendo de uma seqüência artificial, que não aparece no alinhamento. Já a sequiência 6 descende no lado esquerdo de uma (outra) seqüência artificial, que não está presente no alinhamento, e no lado direito ela descende de (um 

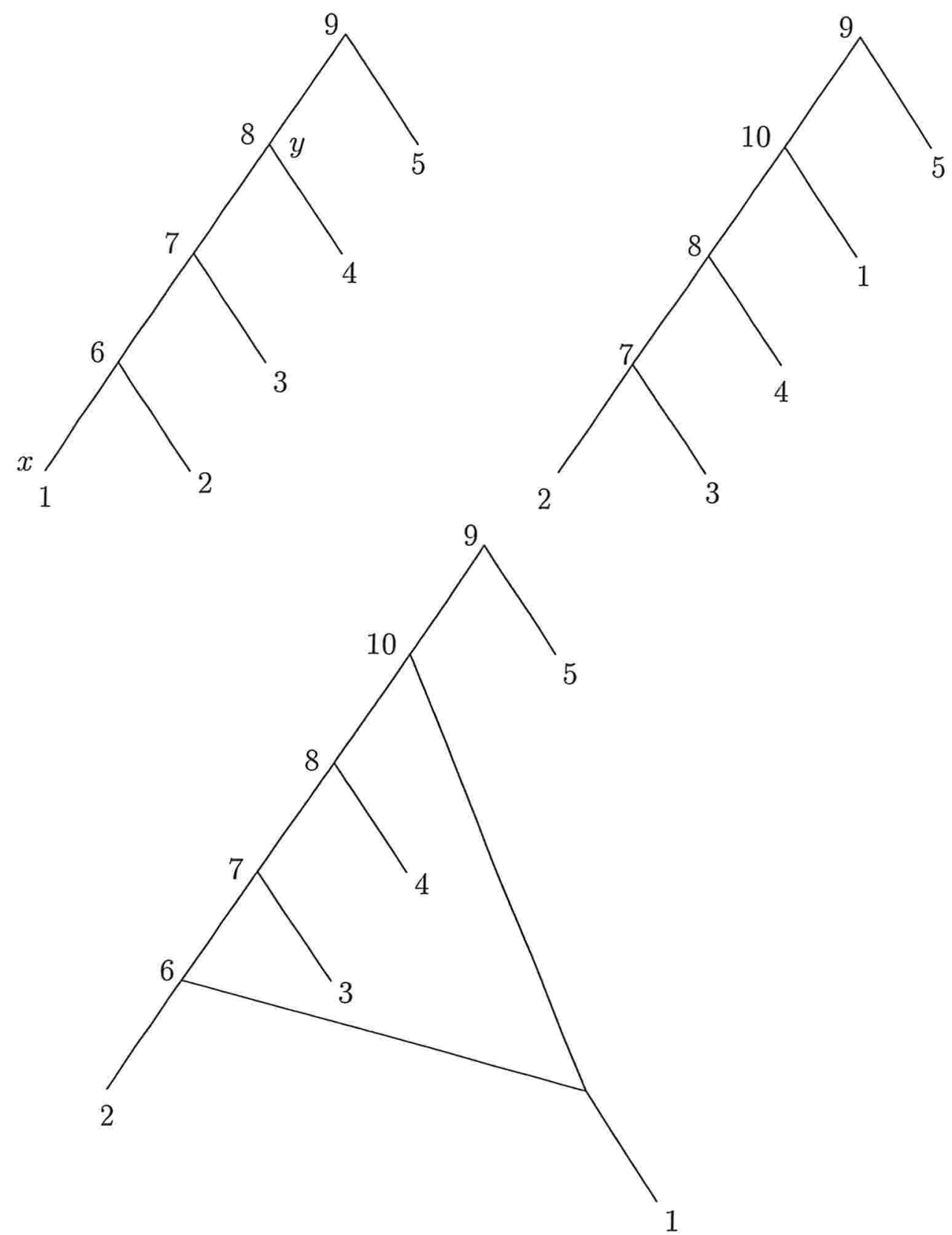

Figura 9.5: Rede para as árvores da Figura 9.4. 

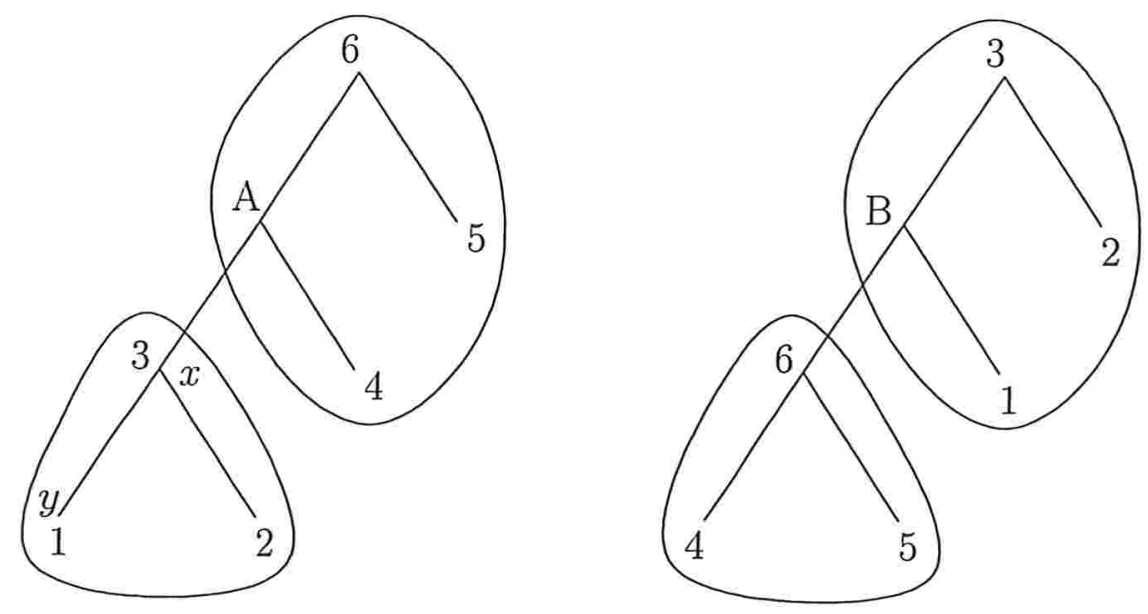

Figura 9.6: Um outro exemplo de árvores derivadas adjacentes cuja rede equivalente apresenta um circuito orientado.

descendente de) B. Podemos ver que aparecem pelo menos três seqüências interagindo por meio de recombinações no alinhamento: $A, B$ e pelo menos uma terceira seqüência diferente de A e de B. Ou seja, temos pelo menos duas recombinações ocorrendo.

Este exemplo mostra uma situação em que temos uma transferência de subárvore correspondendo a dois eventos de recombinação. No caso, a transferência é de tipo TS 5. Portanto, se queremos preservar a correspondência entre eventos de recombinação e transferências de subárvore, a transferência TS5 deve ser proibida.

Vamos voltar neste ponto à questão da ordem entre os lcas dos componentes da floresta de concordância induzida (ver Seção 9.5.1) pelas transferências de subárvore. É possível conservar essa ordem? Em outras palavras, é possível evitar que uma seqüência de transferências de subárvore gere redes com circuitos orientados? O exemplo da Figura 9.8 mostra que o expediente de proibir quaisquer tipos de transferência de subárvore é ineficaz neste caso. A Figura 9.8 mostra a seqüência de transferências de subárvore que transforma a árvore derivada esquerda na árvore derivada direita, e a Figura 9.9 mostra a rede correspondente.

O problema evidente com a rede na Figura 9.9 é que, como no exemplo anterior, ela tem um circuito orientado (seqüências 6-D-9-B-6). Como naquele exemplo, não podemos admitir isso se queremos preservar a ordem no tempo em que as seqüências foram geradas.

A Figura 9.11 mostra um alinhamento correspondente às seqüências na rede da Figura 9.9. Como no exemplo anterior, temos duas recombinações acontecendo, só que desta vez entre dois pares de seqüências claramente distintas: as seqüências A e D produzindo a seqüência 6 , e as seqüências B e C produzindo a seqüência 9. 


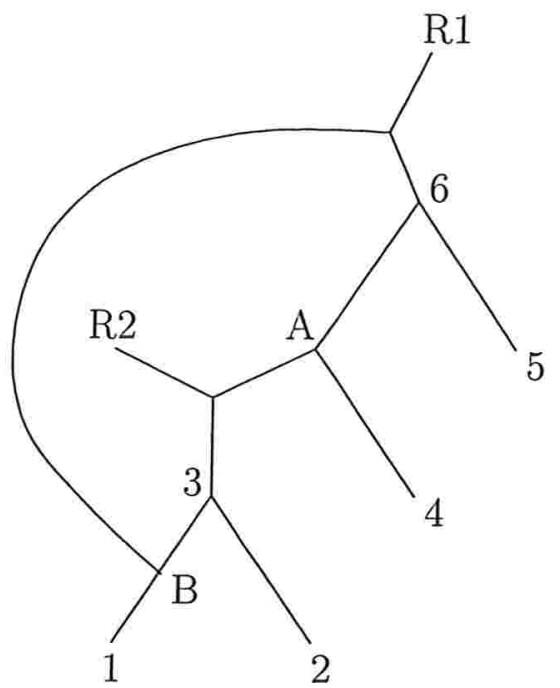

\begin{tabular}{|c|c|c|}
\cline { 2 - 3 } 1 & $\mathrm{~B}$ & $\mathrm{~B}$ \\
\cline { 2 - 3 } 2 & 3 & 3 \\
\cline { 2 - 3 } 3 & $\mathrm{~A}$ & $? ?$ \\
\cline { 2 - 3 } 4 & $\mathrm{~A}$ & $\mathrm{~A}$ \\
\cline { 2 - 3 } 5 & 6 & 6 \\
\cline { 2 - 3 } 6 & $? ?$ & $\mathrm{~B}$ \\
\cline { 2 - 3 } $\mathrm{A}$ & 6 & 6 \\
\cline { 2 - 3 } $\mathrm{B}$ & 3 & 3 \\
\cline { 2 - 3 } & & \multicolumn{2}{|c|}{} &
\end{tabular}

Figura 9.7: Rede e alinhamento para as árvores derivadas da Figura 9.6.
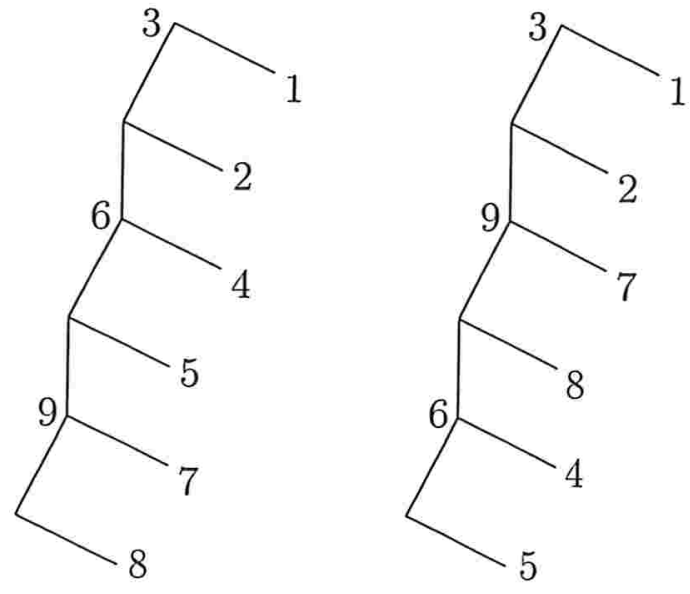

Figura 9.8: Árvores derivadas cuja rede admite circuitos orientados. 


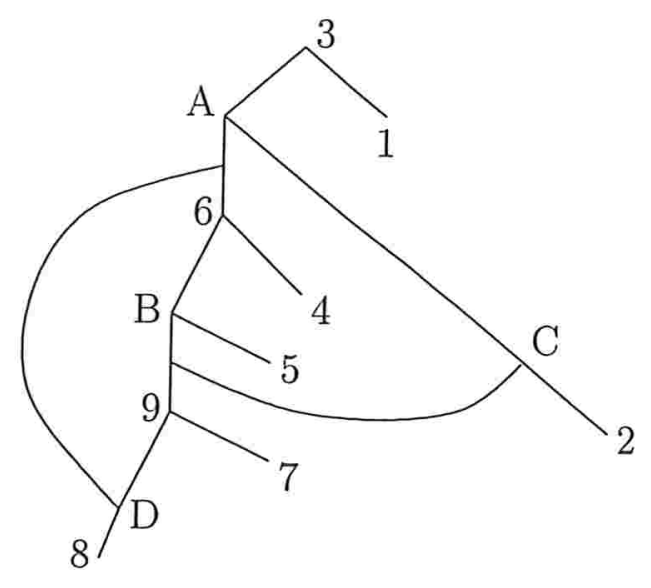

Figura 9.9: Rede para as árvores derivadas na Figura 9.8.
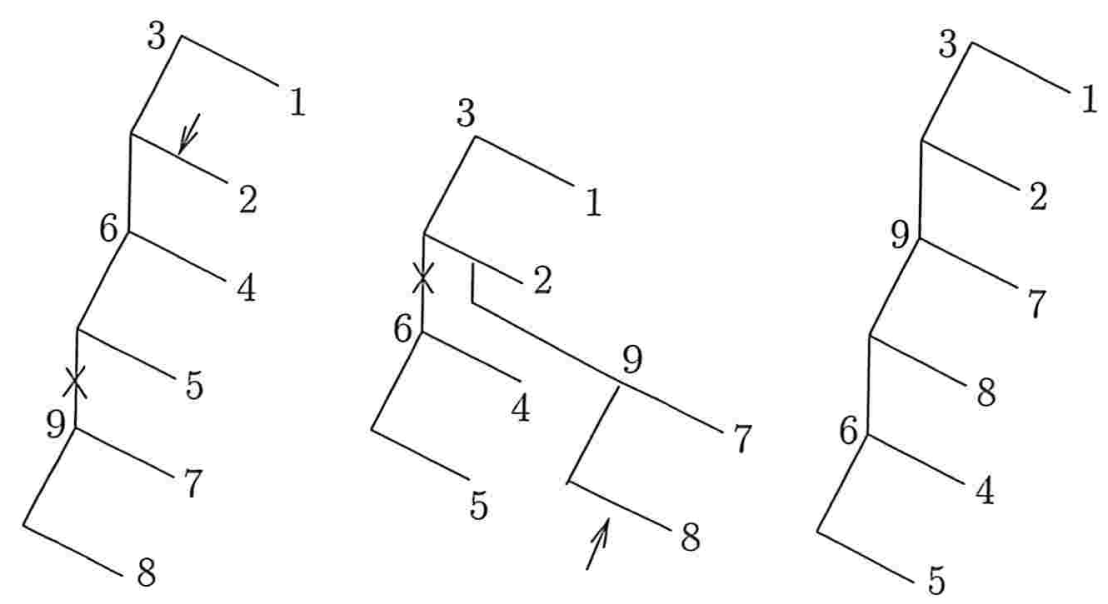

Figura 9.10: Seqüência de transferências de subárvore que transforma a árvore derivada esquerda na árvore derivada direita. 


\begin{tabular}{|l|l|l|l|}
\cline { 2 - 4 } & 3 & 3 & 3 \\
\cline { 2 - 4 } & $\mathrm{C}$ & $\mathrm{C}$ & $\mathrm{C}$ \\
\cline { 2 - 4 } 3 & $? ?$ & $? ?$ & $? ?$ \\
\cline { 2 - 4 } & 6 & 6 & 6 \\
\cline { 2 - 4 } 5 & $\mathrm{~B}$ & $\mathrm{~B}$ & $\mathrm{~B}$ \\
\cline { 2 - 4 } 6 & $\mathrm{~A}$ & $\mathrm{~A}$ & $\mathrm{D}$ \\
\hline 7 & 9 & 9 & 9 \\
\cline { 2 - 4 } & $\mathrm{D}$ & $\mathrm{D}$ & $\mathrm{D}$ \\
\hline 9 & $\mathrm{C}$ & $\mathrm{C}$ & $\mathrm{C}$ \\
\cline { 2 - 4 } $\mathrm{A}$ & 3 & 3 \\
\hline $\mathrm{B}$ & 3 & 6 & 6 \\
$\mathrm{C}$ & 6 & $\mathrm{~A}$ & $\mathrm{~A}$ \\
\cline { 2 - 4 } $\mathrm{D}$ & $\mathrm{A}$ & 9 & 9 \\
\hline
\end{tabular}

Figura 9.11: Alinhamento para as seqüências na Figura 9.9.

\subsection{Transferências padrão}

Na Seção 9.1, foram apresentados os rudimentos do modelo de Hein para eventos de recombinação entre seqüências pré-alinhadas de DNA. Da discussão naquela seção, concluímos que é interessante modelar os eventos de recombinação através de transferências de subárvore. Concluímos ainda que as duas condições seguintes devem ser satisfeitas para o conjunto de transferências de subárvore que vamos adotar para este fim:

- transferências de subárvore definem uma métrica sobre árvores filogenéticas;

- cada transferência de subárvore equivale a um evento de recombinação.

No Capítulo 8 definimos um conjunto de transferências capaz de simular todas as transferências de subárvore possíveis em um passo, e chamamos essas transferências de canônicas. As transferências canônicas satisfazem a primeira das duas propriedades que pedimos de um conjunto de transferências de subárvore que modele eventos de recombinação, já que a inversa de uma transferência canônica é canônica.

Entretanto, ao verificarmos se a segunda condição acima também era respeitada, descobrimos que uma das transferências canônicas, TS 5, não a satisfaz, conforme vimos na Seção 9.2.2. As transferências canônicas restantes, no entanto, satisfazem ambas as propriedades. Vamos denominar, então, as transferências TS 1, TS 2 e TS 3 de transferências padrão. Essas são as transferências que vamos utilizar para resolver o problema do número de pontos de recombinação. 
Vimos na Seção 8.2 que existe uma correspondência entre seqüências de transferências canônicas e florestas de concordância, e que esta correspondência se reduz à equivalência entre duas distâncias entre árvores filogenéticas: MAF e SPR-canônica. Essa equivalência é importante para os nossos propósitos, pois vimos que o problema da floresta de concordância ótima para árvores com grau no máximo 2 é APX-difícil e 3-aproximável. Pela equivalência, esses resultados também são válidos para o cálculo do numero mínimo de transferências canônicas.

Esses resultados fazem da distância canônica (isto é, a distância dada pelas transferências de subárvore canônicas) um problema interessante do ponto de vista teórico. Entretanto, vimos que para os nossos propósitos neste artigo ela não é adequada.

Seria possível conseguirmos um resultado similar para transferências padrão? $\mathrm{Ou}$ ao menos conseguir uma relação entre a distância canônica e a distância padrão (denotada por SPR-padrão) dada pelo número mínimo de transferências de subárvore padrão entre duas árvores filogenéticas, de modo que seja possível aproveitarmos os resultados sobre a distância canônica para atacar o problema do número de pontos de recombinação? As próximas seções tratam de responder a estas questões.

\subsection{Limitante inferior para a distância padrão}

Um limitante inferior para a distância padrão que utiliza a distância canônica pode ser obtido da seguinte propriedade:

Lema 9.1 Toda transferência de subárvore do tipo TS5 pode ser simulada por duas transferências de subárvore dos tipos TS2 e TS3.

Prova. Veja a Figura 9.12.

Uma primeira conseqüência desta propriedade é que, dadas duas árvores filogenéticas quaisquer, a distância padrão entre elas sempre está definida. Pelo Lema 8.1, a distância canônica está definida para qualquer par $\mathcal{T}, \mathcal{U}$ de árvores filogenéticas. Portanto, em qualquer seqüência de transferências canônicas que transforme $\mathcal{T}$ em $\mathcal{U}$ basta substituir as operações TS 5 por operações TS 2 e TS 3 de acordo com a Figura 9.12 para obtermos uma seqüência de transferências padrão que transforma $\mathcal{T}$ em $\mathcal{U}$.

Outra conseqüência é que a distância canônica é um limitante inferior para a distância padrão. Isto é dado pelo seguinte resultado, cuja prova é imediata:

Teorema 9.2 Sejam $\mathcal{T}$ e $\mathcal{U}$ duas árvores filogenéticas com $S_{\mathcal{T}}=S_{\mathcal{U}}$. Então

$$
\operatorname{SPR} \text {-canônica }(\mathcal{T}, \mathcal{U}) \leq \operatorname{SPR} \text {-padrão }(\mathcal{T}, \mathcal{U}) \text {. }
$$



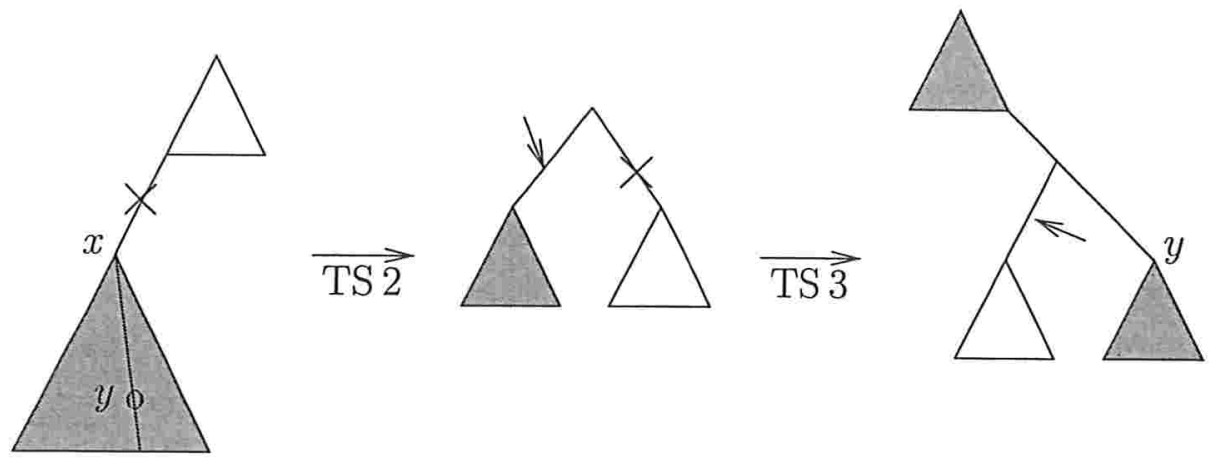

Figura 9.12: Simulação de TS 5 usando TS 2 e TS 3.

\subsection{Limitante superior para a distância padrão}

\subsubsection{Florestas de concordância induzidas por seqüências de trans- ferências de subárvore}

Vamos definir essa noção por indução no número de operações empregadas nas seqüências de transferência.

Sejam $\mathcal{T}$ e $\mathcal{U}$ duas árvores filogenéticas com $S_{\mathcal{T}}=S_{\mathcal{U}}$, e suponha que $\left(T_{1}, \ldots, T_{m}\right)$ é uma seqüência de transferências canônicas de subárvore que transforma $\mathcal{T}$ em $\mathcal{U}$. Observe que uma tal seqüência pode existir mesmo nos casos em que $\mathcal{T}=\mathcal{U}$. Seja $\mathcal{T}_{0}:=\mathcal{T}$ e, para cada $i, 1 \leq i \leq m$, seja $\mathcal{T}_{i}$ o resultado da aplicação de $T_{i}$ a $\mathcal{T}_{i-1}$. Por essa definição temos $\mathcal{T}_{m}=\mathcal{U}$. Vamos convencionar que $\mathcal{T}$ é sempre o resultado da aplicação de uma seqüência vazia de transferências de subárvore a $\mathcal{T}$, para qualquer árvore filogenética $\mathcal{T}$.

Para todo $i=1, \ldots, m$, definimos a floresta de concordância induzida pela seqüência de transferências $\left(T_{1}, \ldots, T_{i}\right)$ como sendo a floresta $\mathcal{F}_{i}$ obtida a partir da floresta induzida $\mathcal{F}_{i-1}$ do seguinte modo:

Sejam $x$ e $y$ os nós de $\mathcal{T}_{i-1}$ tais que $T_{i}=\operatorname{TS}\left(\mathcal{T}_{i-1}, x, y\right)$. Se o arco $e$ que sai de $x$ é de ligação com relação a $\mathcal{F}_{i-1}$, então $\mathcal{F}_{i}:=\mathcal{F}_{i-1}$, caso contrário $\mathcal{S}_{\mathcal{F}_{i}}:=\mathcal{S}_{\mathcal{F}_{i-1}} \backslash$ $\left\{S_{\mathcal{V}}\right\} \cup\{D(x)\} \cup\left\{S_{\mathcal{V}} \backslash D(x)\right\}$ onde $\mathcal{V}$ é o componente de $\mathcal{F}_{i-1}$ que contém e. Veja a Figura 9.13.

O seguinte lema será útil nas próximas seções:

Lema 9.3 Sejam $\mathcal{T}$ e $\mathcal{U}$ duas árvores fllogenéticas com $S_{\mathcal{T}}=S_{\mathcal{U}}$. Se uma seqüência de transferências canônicas de subárvore que transforma $\mathcal{T}$ em $\mathcal{U}$ tem comprimento $m$, então a floresta de concordância induzida $\mathcal{F}_{m}$ por essa seqüência tem tamanho no máximo $m+1$. 


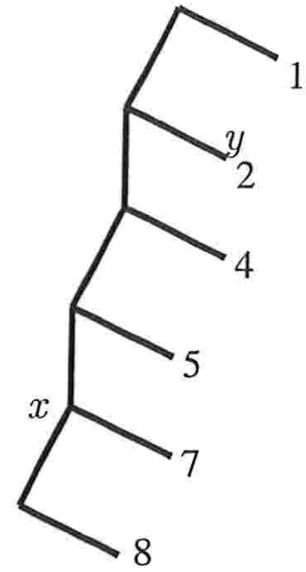

$\mathcal{S}_{\mathcal{F}_{0}}=\{\{1,2,4,5,7,8\}\}$

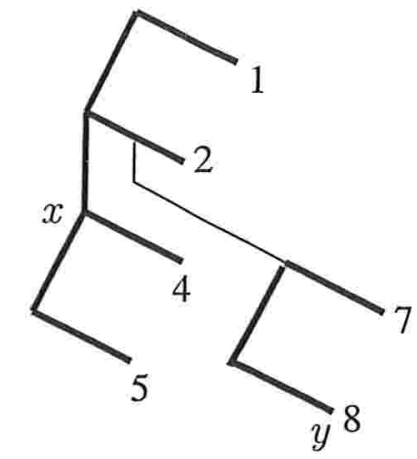

$\mathcal{S}_{\mathcal{F}_{1}}=\{\{7,8\},\{1,2,4,5\}\}$

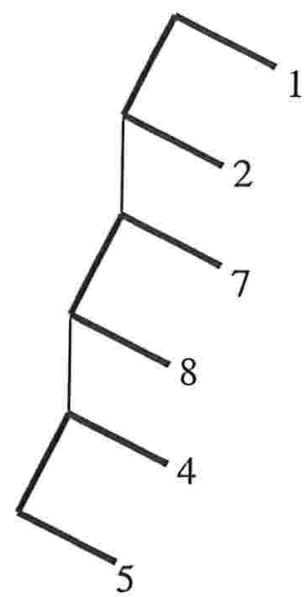

$\mathcal{S}_{\mathcal{F}_{2}}=\{\{7,8\},\{4,5\},\{1,2\}\}$

Figura 9.13: $\mathcal{F}_{i}$ (traços escuros) e $\mathcal{S}_{\mathcal{F}_{i}}$ para a seqüência de transferências da Figura 9.10 .

Prova. Vamos provar esse lema por indução em $m$.

Se $m=0$ então $\mathcal{F}_{m}=\mathcal{F}_{0}=\mathcal{T}$ e portanto $\left|\mathcal{F}_{m}\right|=1=m+1$.

Se $m \geq 1$, temos $\left|\mathcal{F}_{m-1}\right| \leq m$ por hipótese de indução. Considere os dois casos possíveis da definição de $\mathcal{F}_{m}$. Se $\mathcal{S}_{\mathcal{F}_{m}}$ é definida como $\mathcal{S}_{\mathcal{F}_{m-1}}$ então $\mathcal{F}_{m}=\mathcal{F}_{m-1}$ e $\left|\mathcal{F}_{m}\right|=\left|\mathcal{F}_{m-1}\right| \leq m$. Se $\mathcal{S}_{\mathcal{F}_{m}}$ é definida como $\mathcal{S}_{\mathcal{F}_{m-1}} \backslash\left\{S_{\mathcal{V}}\right\} \cup\{D(x)\} \cup\left\{S_{\mathcal{V}} \backslash D(x)\right\}$ então:

$$
\begin{aligned}
\left|\mathcal{F}_{m}\right|=\left|\mathcal{S}_{\mathcal{F}_{m}}\right| & = \\
\left|\mathcal{S}_{\mathcal{F}_{m-1}} \backslash\left\{S_{\mathcal{V}}\right\} \cup\{D(x)\} \cup\left\{S_{\mathcal{V}} \backslash D(x)\right\}\right| & = \\
\left|\mathcal{S}_{\mathcal{F}_{m-1}}\right|+1=\left|\mathcal{F}_{m-1}\right|+1 & \leq m+1 .
\end{aligned}
$$

Observamos que esta prova é similar em muitos aspectos à prova da primeira parte do Lema 8.1.

\subsubsection{Restrições induzidas por florestas de concordância}

Antes de seguirmos adiante com os resultados que relacionam a distância padrão à distância canônica, precisamos apresentar mais alguns conceitos novos relacionados às noções de restrição e de floresta de concordância.

Os conceitos de restrição e de floresta de concordância capturam a noção intuitiva de regiões "parecidas", em topologia e rotulação das folhas, entre duas florestas rotuladas. De fato, se $\mathcal{G}$ e $\mathcal{H}$ são duas florestas rotuladas e $\mathcal{F}$ é uma floresta de 

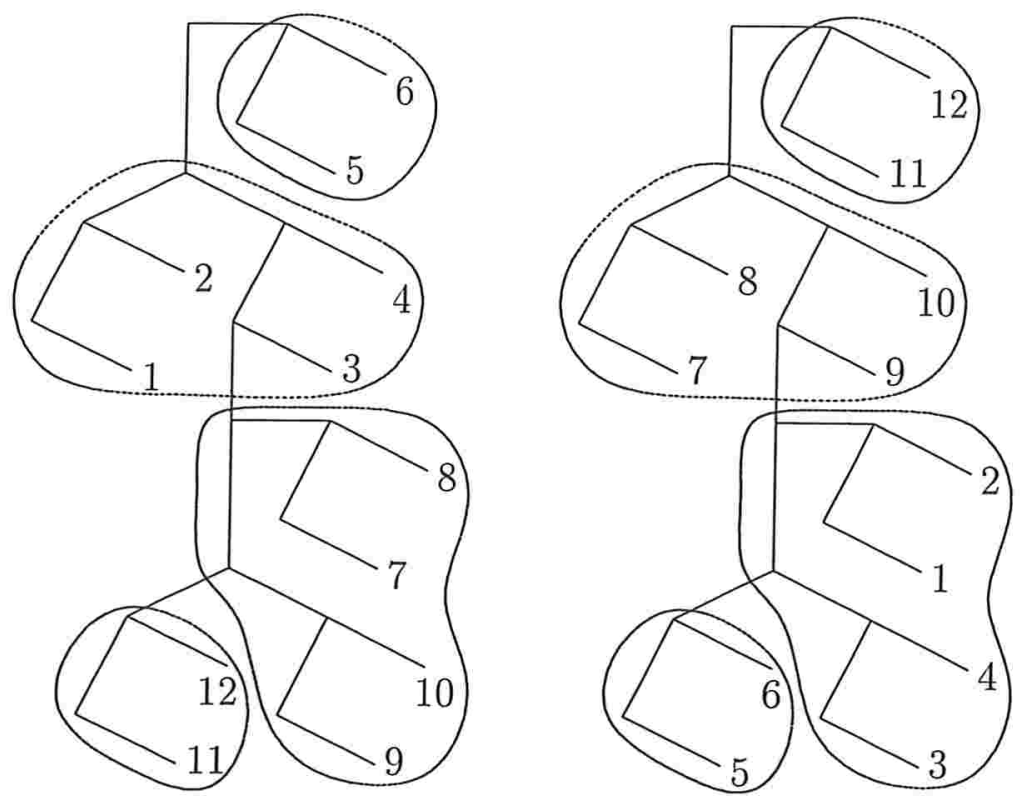

Figura 9.14: Componente bloqueados e não bloqueados com relação a uma floresta de concordância ótima de um par de árvores filogenéticas.

concordância de $\mathcal{G}$ e $\mathcal{H}$, então por definição $\mathcal{G} \mid \mathcal{S}_{\mathcal{F}}$ e $\mathcal{H} \mid \mathcal{S}_{\mathcal{F}}$ são isomorfas. Lembramos que denominamos as restrições $\mathcal{G} \mid \mathcal{S}_{\mathcal{F}}$ e $\mathcal{H} \mid \mathcal{S}_{\mathcal{F}}$ restrições induzidas por $\mathcal{F}$ sobre $\mathcal{G}$ e $\mathcal{H}$, respectivamente.

Nesta seção, entretanto, queremos não apenas localizar regiões "parecidas" entre árvores filogenéticas mas também manipular e transformar as "conexões" entre essas regiões. Essas "conexões" são dadas pelos arcos de ligação que conectam os componentes das restrições induzidas por florestas de concordância.

Seja $\mathcal{T}$ uma árvore filogenética, $\mathcal{W}$ uma restrição completa de $\mathcal{T}$, e $\mathcal{W}_{1}$ um componente de $\mathcal{W}$. Dizemos que $\mathcal{W}_{1}$ está bloqueado com relação a $\mathcal{W}$ se existe um componente $\mathcal{W}_{2}$ de $\mathcal{W}$ tal que $\mathcal{W}_{2} \neq \mathcal{W}_{1}$ e $r_{\mathcal{W}_{2}}$ pertence ao caminho que conecta $r_{\mathcal{W}_{1}}$ a $r_{\mathcal{W}}$. Neste caso dizemos que $\mathcal{W}_{2}$ bloqueia $\mathcal{W}_{1}$ com relação a $\mathcal{W}$.

A Figura 9.14 exemplifica essas definições. Na árvore da esquerda os componentes induzidos por $\{11,12\}$ e $\{7,8,9,10\}$ estão bloqueados, enquanto que os componentes induzidos por $\{1,2,3,4\}$ e $\{5,6\}$ não estão. Na árvore da direita os componentes bloqueados são induzidos por $\{5,6\}$ e $\{1,2,3,4\}$ e os componentes não bloqueados são induzidos por $\{11,12\}$ e $\{7,8,9,10\}$. 


\subsubsection{Relação entre a distância padrão e a distância canônica}

De modo análogo ao que fizemos para a distância canônica, apresentamos aqui um algoritmo que constrói uma seqüência de transferências de subárvore padrão a partir de uma floresta de concordância.

\section{Algoritmo TransfPad}

Entrada: Duas árvores filogenéticas $\mathcal{T}$ e $\mathcal{U}$ tais que $S_{\mathcal{T}}=S_{\mathcal{U}}$ e uma floresta de concordância ótima $\mathcal{F}$ de $\mathcal{T}$ e $\mathcal{U}$;

Saída: Seqüência de transferências de subárvore com no máximo $|\mathcal{F}|$ operações padrão que transforma $\mathcal{T}$ em $\mathcal{U}$.

(1) $\mathcal{T}^{\prime}:=\mathcal{T} \mid \mathcal{S}_{\mathcal{F}}$

(2) $\mathcal{U}^{\prime}:=\mathcal{U} \mid \mathcal{S}_{\mathcal{F}}$

(3) Enquanto $\mathcal{U}^{\prime}$ possui mais do que um componente

(4) Se $\mathcal{U}^{\prime}$ possui um par de componentes $\mathcal{U}_{1}, \mathcal{U}_{2}$

tal que lca ${ }_{\mathcal{U}}\left(r_{\mathcal{U}_{1}}, r_{\mathcal{U}_{2}}\right)$ não pertence a nenhum componente de $\mathcal{U}^{\prime}$ então $\{$ Caso 1$\}$

$$
\begin{aligned}
& \text { Sejam } \mathcal{U}_{1} \text { e } \mathcal{U}_{2} \text { dois tais componentes tais que } \\
& r_{\mathcal{U}_{1}} \text { e } r_{\mathcal{U}_{2}} \text { têm distância mínima; }
\end{aligned}
$$

Sejam $\mathcal{T}_{1}$ e $\mathcal{T}_{2}$ os componentes de $\mathcal{T}^{\prime}$ isomorfos respectivamente a $\mathcal{U}_{1}$ e $\mathcal{U}_{2}$;

Se $\operatorname{lca} \mathcal{T}_{\mathcal{T}}\left(r_{\mathcal{T}_{1}}, r_{\mathcal{T}_{2}}\right) \notin\left\{r_{\mathcal{T}_{1}}, r_{\mathcal{T}_{2}}\right\}$ então

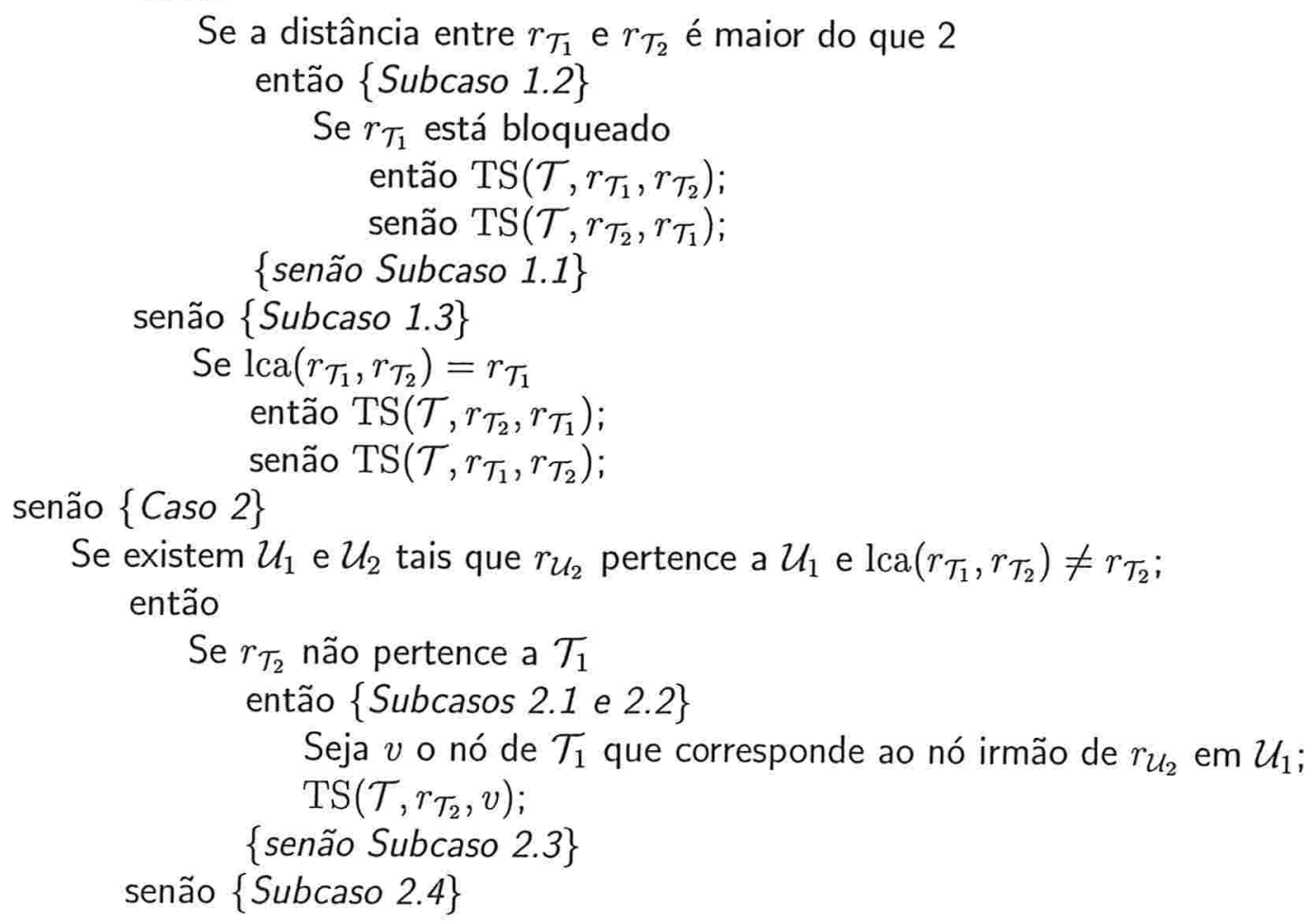
senão $\{$ Caso 2$\}$ 


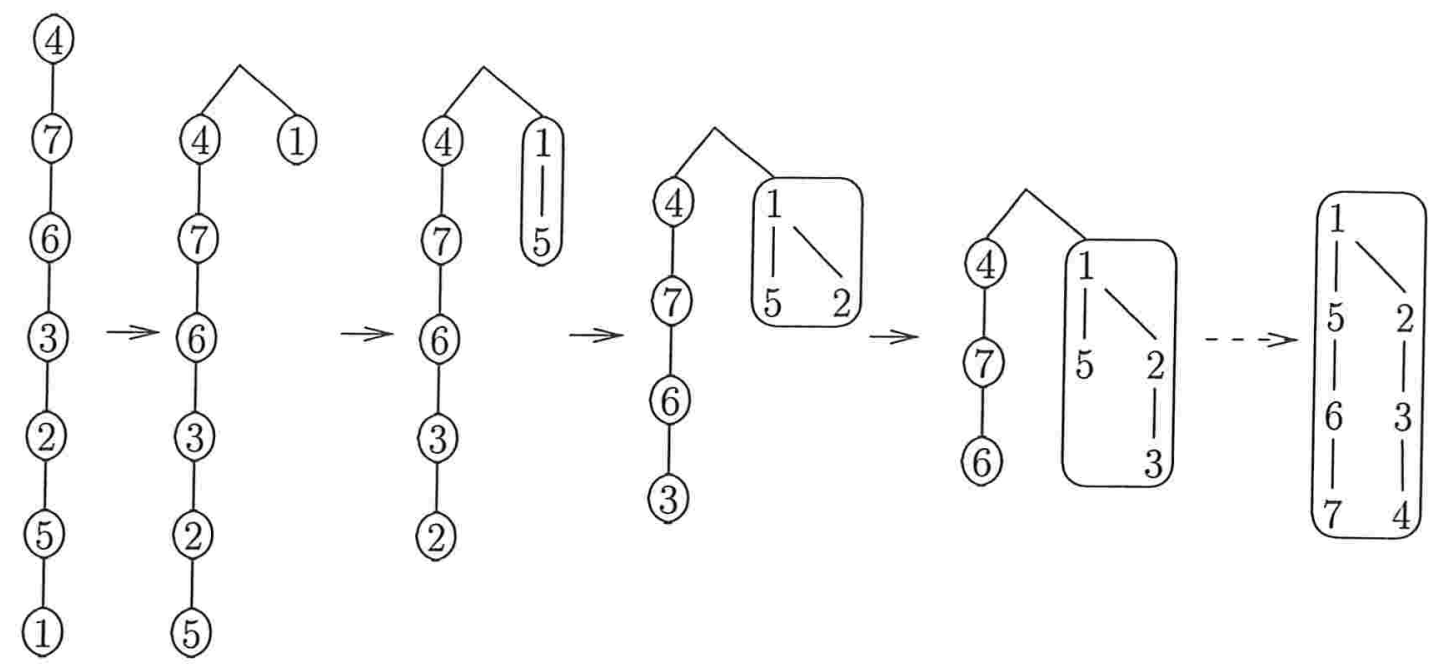

Figura 9.15: Um exemplo da execução do Algoritmo 4.

(21) Atualize $\mathcal{F}$;

Sejam $\mathcal{U}_{1}, \mathcal{U}_{2}$ tais que $r_{\mathcal{T}_{1}}=r_{\mathcal{T}}$ e $r_{\mathcal{T}_{2}}$ tem profundidade máxima dentre os componentes de $\mathcal{T}^{\prime}$;

(22) $\quad \mathcal{T}^{\prime}:=\mathcal{T} \mid \mathcal{S}_{\mathcal{F}}$; $\mathrm{TS}\left(\mathcal{T}, r_{\mathcal{T}_{2}}, r_{\mathcal{T}_{1}}\right)$

(23) $\quad \mathcal{U}^{\prime}:=\mathcal{U} \mid \mathcal{S}_{\mathcal{F}}$;

(24) Imprima todas as transferências de subárvore realizadas.

Este algoritmo é muito semelhante ao Algoritmo TransfCan, que calcula a seqüência de transferências canônicas, exceto pelas linhas 9 a 11, que correspondem à linha 9 de TransfCan, e pelas linhas 19 e 20, que corresponde às linhas 17 a 20 de TransfCan. Os casos indicados nos comentários são os mesmos definidos na prova da segunda parte do Lema 8.1. Um exemplo da execução deste algoritmo é dado na Figura 9.15. Nesta figura, representamos as sucessivas árvores que são obtidas a partir de $\mathcal{T}$ (primeira árvore à esquerda) durante a execução do algoritmo, até a última árvore, que é $\mathcal{U}$ (árvore mais à direita).

A atualização da floresta de concordância $\mathcal{F}$ no Algoritmo TransfPad é basicamente a mesma que é feita para o Algoritmo TransfCan, exceto que nas iterações em que as linhas 19 e 20 são executadas, $\mathcal{F}$ não muda.

Este procedimento de atualização de $\mathcal{F}$ não conserva a propriedade-chave para entendermos o funcionamento do Algoritmo TransfCan: o tamanho de $\mathcal{F}$ diminui em uma unidade a cada iteração. Com base nesta propriedade é que garantimos que a seqüência de operações de transferência devolvida pelo Algoritmo TransfCan tem no máximo $|\mathcal{F}|-1$ operações. No Algoritmo TransfPad isto não ocorre nas iterações 
em que as linhas 19 e 20 são executadas, embora ocorra em todos os demais casos. Portanto, em princípio, o Algoritmo TransfPad pode devolver seqüências de operações muito mais longas. Entretanto, podemos verificar facilmente que nesse algoritmo as linhas 19 e 20 só podem ser alcançadas uma única vez. Portanto, esse algoritmo devolve uma seqüência de no máximo $|\mathcal{F}|$ operações.

A prova de que o Algoritmo TransfPad está correto demonstra que SPR-padrão $(\mathcal{T}, \mathcal{U}) \leq \operatorname{MAF}(\mathcal{T}, \mathcal{U})$. Este fato mais os Teoremas 8.2 e 9.2 demonstram o seguinte resultado:

Teorema 9.4 Sejam $\mathcal{T}$ e $\mathcal{U}$ árvores filogenéticas com $S_{\mathcal{T}}=S_{\mathcal{U}}$. Então temos que $\operatorname{SPR}$-padrão $(\mathcal{T}, \mathcal{U})=\operatorname{MAF}(\mathcal{T}, \mathcal{U})-1$ ou $\operatorname{SPR}$-padrão $(\mathcal{T}, \mathcal{U})=\operatorname{MAF}(\mathcal{T}, \mathcal{U})$.

Provamos neste trabalho que a distância MAF é 3-aproximável. Como conseqüência desse fato e do cálculo final da razão de aproximação do problema MAF-2, temos que:

Teorema 9.5 As distâncias SPR-canônica e SPR-padrão são 3-aproximáveis. 


\section{Considerações finais}

Nesta seção, tecemos alguns comentários sobre o trabalho desenvolvido e suas contribuições, e sobre possíveis direções para pesquisa futura.

Para analisar as razões de aproximação dos Algoritmos $\mathcal{A}_{1}$ e $\mathcal{A}_{2}$, desenvolvemos a partir das idéias de Hein et al. [19, 20] uma técnica para fazer uma contagem amortizada do número de operações de corte realizadas ao longo da execução desses algoritmos. (Lembramos que o número de operações de corte está intimamente ligado com o número de componentes das soluções construídas pelos algoritmos.) Técnicas de análise amortizada são muito utilizadas na determinação da complexidade de algoritmos [12]. Seria interessante tentar explorar a viabilidade da aplicação dessa técnica para a obtenção de limitantes superiores para a razão de aproximação de algoritmos para outros problemas. Esta técnica é comumente empregada na análise de algoritmos online (Borodin [7]).

As análises que desenvolvemos para os algoritmos de 3 -aproximação $\mathcal{A}_{2}, \mathcal{B}$ e $\mathcal{C}$ não evidenciam a grande diferença na qualidade das soluções que esses algoritmos podem construir. Essa diferença ficou evidente apenas nos testes com as implementações dos algoritmos, relatados no Capítulo 5. Em particular, observamos que as soluções encontradas pelo Algoritmo $\mathcal{A}_{2}$, cuja análise é a mais complicada dentre os três algoritmos, foram sistematicamente as mais próximas do ótimo.

A discussão sobre os diferentes tipos de distância entre árvores filogenéticas baseadas em transferências de subárvore e sobre a equivalência entre tipos de transferência de subárvore e eventos de recombinação, feita nos Capítulos 8 e 9 deste trabalho, supre uma deficiência que observamos em nosso levantamento bibliográfico. A importância de uma formulação precisa da definição de transferência de subárvore fica clara quando consideramos a observação de Allen e Steel [1], de que as distâncias dadas pelo tamanho de uma floresta de concordância ótima e por uma seqüência de transferências de subárvore não são equivalentes para a noção de transferência de subárvore proposta por Hein [17, 18] para modelar eventos de recombinação.

O trabalho desenvolvido aqui insere-se no contexto de distâncias entre árvores filogenéticas, ao qual foi dispendido um certo esforço de pesquisa nos últimos anos. Foram propostos algoritmos exatos e de aproximação para o cálculo de distâncias 
como NNI, SPR e TBR $[13,27,22,28,8,21,1]$. Seria interessante explorarmos as relações entre a distância MAF e algumas dessas distâncias, tanto para árvores filogenéticas enraizadas quanto para árvores filogenéticas não enraizadas.

Algumas outras questões que podem ser pesquisadas em continuação a este trabalho, são:

- O bom desempenho do Algoritmo $\mathcal{A}_{2}$ mantém-se em testes com pares de árvores filogenéticas obtidas a partir de dados sequenciais pré-alinhados reais?

- É possível obter um algoritmo para o problema MAF-2 com razão de aproximação melhor do que 3 ? Se sim, este algoritmo pode ser estendido para o problema MAF- $d$, a exemplo do que foi feito com o Algoritmo $\mathcal{A}_{2}$ ?

- Qual a complexidade do problema MAF, em que não existe um limitante superior no grau dos nós das árvores dadas como entrada? Existem algoritmos de aproximação com razão constante para este problema?

- Qual a complexidade do problema de calcular $\operatorname{SPR}$-padrão $(\mathcal{T}, \mathcal{U})$ para duas árvores filogenéticas $\mathcal{T}$ e $\mathcal{U}$ ? Para quais instâncias $\mathcal{T}, \mathcal{U}$ temos

$$
\operatorname{SPR} \text {-padrão }(\mathcal{T}, \mathcal{U})=\operatorname{MAF}(\mathcal{T}, \mathcal{U})
$$

e para quais temos

$$
\operatorname{SPR} \text {-padrão }(\mathcal{T}, \mathcal{U})=\operatorname{MAF}(\mathcal{T}, \mathcal{U})-1 ?
$$




\section{Referências Bibliográficas}

[1] B. Allen and M. Steel. Subtree transfer operations and their induced metrics on evolutionary trees. Annals of Combinatorics, 5:1-13, 2001.

[2] S. Arora, C. Lund, R. Motwani, M. Sudan, and M. Szegedy. Proof verification and hardness of approximation problems. In Proceedings of the 33rd IEEE Symposium on the Foundations of Computer Science, 1992.

[3] G. Ausiello, P. Crescenzi, G. Gambosi, V. Kann, A. Marchetti-Spaccamella, and M. Protasi. Complexity and Approximation: Combinatorial Optimization Problems and Their Approximability Properties. Springer Verlag, 1999.

[4] C. Berge. Graphs. North-Holland, 1991.

[5] P. Berman and T. Fujito. On approximation properties of the independent set problem for degree 3 graphs. In Proceedings of the 4 th Workshop on Algorithms and Data Structures, 1995.

[6] J. A. Bondy and U. S. R. Murty. Graph Theory with Applications. MacMillan, 1976.

[7] A. Borodin and R. El-Yaniv. Online computation and competitive analysis. Cambridge University Press, 1998.

[8] D. Bryant, J. Tsang, P. Kearney, and M. Li. Computing the quartet distance between evolutionary trees. In Proceedings of the 11th ACM-SIAM Symposium of Discrete Algorithms, 2000.

[9] V. Chvátal. Linear Programming. W. H. Freeman and Company, 1983.

[10] A. Cobham. The intrinsic computational difficulty of functions. In Y. BarHillel, editor, Proceedings of the International Congress for Logic Methodology and Philosophy of Science, 1964.

[11] S. A. Cook. The complexity of theorem-proving procedures. In Proceedings of the 3rd ACM Symposium on Theory of Computing, 1971. 
[12] T. H. Cormen, C. E. Leiserson, and R. L. Rivest. Introduction to Algorithms. MIT Press, 1990.

[13] B. dasGupta, X. He, T. Jiang, M. Li, and J. Tromp. On the linear-cost subtreetransfer distance between phylogenetic trees. Algorithmica, 25:427-436, 1999.

[14] J. Edmonds. Paths, trees and flowers. Canadian Journal of Mathematics, 17:449467, 1965.

[15] C. G. Fernandes, F. K. Miyazawa, , M. Cerioli, and P. Feofiloff, editors. Uma introdução sucinta a algoritmos de aproximação. Instituto de Matemática Pura e Aplicada, 2001.

[16] M. R. Garey and D. S. Johnson. Computers and Intractability. W. H. Freeman and Company, 1979.

[17] J. Hein. Reconstructing evolution of sequences subject to recombination using parsimony. Mathematical Biosciences, 98:185-200, 1990.

[18] J. Hein. A heuristic method to reconstruct the history of sequences subject to recombination. Journal of Molecular Evolution, 36:396-405, 1993.

[19] J. Hein, T. Jiang, L. Wang, and K. Zhang. On the complexity of comparing evolutionary trees. In Proceedings of the 6th Annual Symposium on Combinatorial Pattern Matching, volume 937 of Lecture Notes in Computer Science. Springer Verlag, 1995.

[20] J. Hein, T. Jiang, L. Wang, and K. Zhang. On the complexity of comparing evolutionary trees. Discrete Applied Mathematics, 71:153-169, 1996.

[21] W. K. Hon, M. Y. Kao, and T. W. Lam. Improved phylogeny comparisons: nonshared edges, nearest neighbor interchanges, and subtree transfers. In Proceedings of the 11th Annual International Symposium on Algorithms and Computation (ISAAC '00), 2000.

[22] W. K. Hon and T. W. Lam. Approximating the nearest neighbor interchanging distance for evolutionary trees with non-uniform degrees. In Proceedings of the 5th COCOON, volume 1627 of Lecture Notes in Computer Science. Springer Verlag, 1999.

[23] R. M. Karp. Reducibility among combinatorial problems. In R. E. Miller and J. W. Thatcher, editors, Complexity of Computer Computations, pages 85-103. Plenum Press, 1972.

[24] J. Kececioglu and D. Gusfield. Reconstructing a history of recombinations from a set of sequences. In Proceedings of the 5th ACM-SIAM Symposium on Discrete Algorithms, pages 471-480, 1994. 
[25] S. Khanna, H. Motwani, M. Sudan, and U. Vazirani. On syntactic versus computational views of approximability. SIAM Journal on Computing, 28(1):164-191, 1999.

[26] D. Knuth. The Art of Computer Programming, Vol. 1. Addison-Wesley Publishing Company, 1973.

[27] M. Li, J. Tromp, and L. Zhang. On the nearest neighbour interchange distance between evolutionary trees. Journal on Theoretical Biology, 182(4):463-467, 1996.

[28] M. Li and L. X. Zhang. Twist-rotation transformations of binary trees and aritmetic expressions. Journal of Algorithms, 32(2):155-166, 1999.

[29] B. Ma, L. Wang, and M. Li. Fixed topology alignment with recombination. In Proceedings of the 9th Annual Symposium on Combinatorial Pattern Matching, volume 1448 of Lecture Notes in Computer Science. Springer Verlag, 1998.

[30] C. Papadimitriou. Computational Complexity. Addison-Wesley Publishing Company, 1994.

[31] E. M. Rodrigues, M.-F. Sagot, and Y. Wakabayashi. Some approximation results for the maximum agreement forest problem. In Proceedings of the 4th International Workshop on Approximation Algorithms for Combinatorial Optimization Problems, volume 2129 of Lecture Notes in Computer Science. Springer Verlag, 2001.

[32] J. C. Setubal and J. Meidanis. Introduction to Computational Molecular Biology. PWS Publishing Company, 1996.

[33] D. L. Swofford, G. J. Olsen, P. J. Waddell, and D. H. Hillis. Phylogenetic inference. In D. Hillis, C. Moritz, and B. Mable, editors, Molecular Systematics. Sinauer Associates, 1996. 